\title{
Californium-252 Program Equipment Evaluation
}

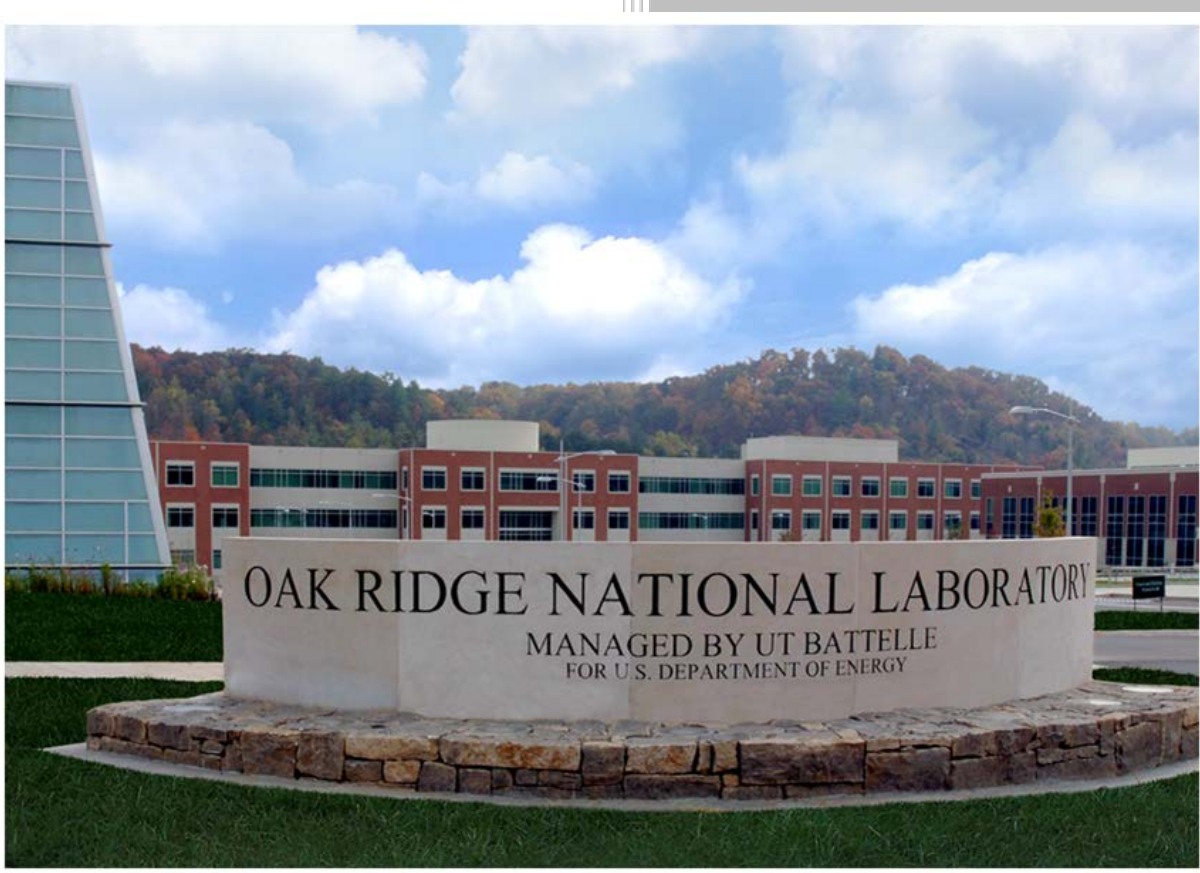

Approved for public release. Distribution is unlimited.

Julie G. Ezold Fred R. Chattin Kenton Wilson

January 2018 


\title{
DOCUMENT AVAILABILITY
}

Reports produced after January 1, 1996, are generally available free via US Department of Energy (DOE) SciTech Connect.

\section{Website http://www.osti.gov/scitech/}

Reports produced before January 1, 1996, may be purchased by members of the public from the following source:

\author{
National Technical Information Service \\ 5285 Port Royal Road \\ Springfield, VA 22161 \\ Telephone 703-605-6000 (1-800-553-6847) \\ TDD 703-487-4639 \\ Fax 703-605-6900 \\ E-mail info@ntis.gov \\ Website http://www.ntis.gov/help/ordermethods.aspx
}

Reports are available to DOE employees, DOE contractors, Energy Technology Data Exchange representatives, and International Nuclear Information System representatives from the following source:

Office of Scientific and Technical Information

PO Box 62

Oak Ridge, TN 37831

Telephone 865-576-8401

Fax 865-576-5728

E-mail reports@osti.gov

Website http://www.osti.gov/contact.html

This report was prepared as an account of work sponsored by an agency of the United States Government. Neither the United States Government nor any agency thereof, nor any of their employees, makes any warranty, express or implied, or assumes any legal liability or responsibility for the accuracy, completeness, or usefulness of any information, apparatus, product, or process disclosed, or represents that its use would not infringe privately owned rights. Reference herein to any specific commercial product, process, or service by trade name, trademark, manufacturer, or otherwise, does not necessarily constitute or imply its endorsement, recommendation, or favoring by the United States Government or any agency thereof. The views and opinions of authors expressed herein do not necessarily state or reflect those of the United States Government or any agency thereof. 
Nuclear Security and Isotope Technology Division

\title{
CALIFORNIUM-252 PROGRAM EQUIPMENT EVALUATION
}

\author{
Julie G. Ezold \\ Fred R. Chattin \\ Kenton Wilson
}

Date Published: November 2017

Prepared by

OAK RIDGE NATIONAL LABORATORY

Oak Ridge, TN 37831-6283

managed by

UT-BATTELLE, LLC

for the

US DEPARTMENT OF ENERGY

under contract DE-AC05-00OR22725 



\section{CONTENTS}

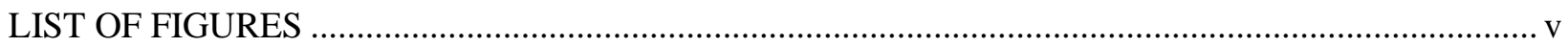

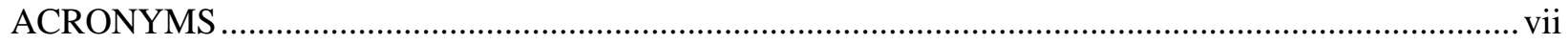

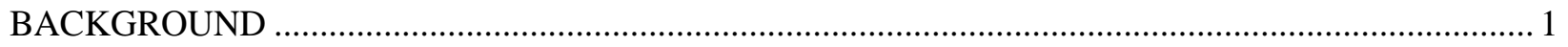

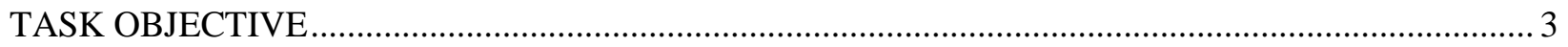

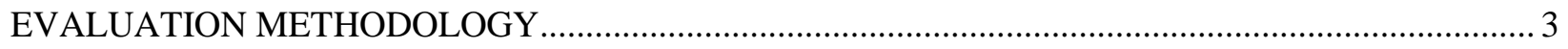

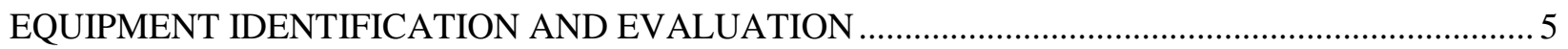

1.0 Target Rod Dissolution (Including Target Rod Hex Can Removal and Scrap Al Dissolution)......... 5

1.1 Target Rod Hex Can Removal .......................................................................................... 5

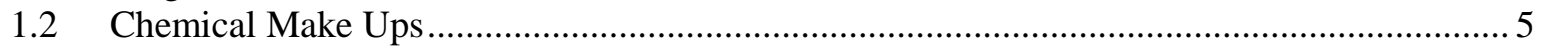

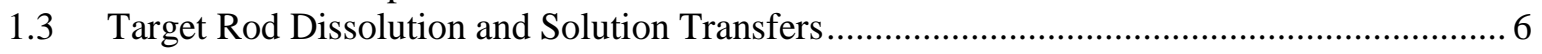

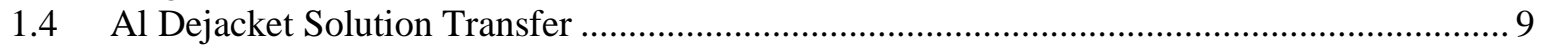

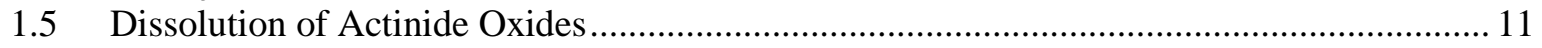

1.6 Actinide Product Solution Transfer from T-70 to T-72 …............................................ 12

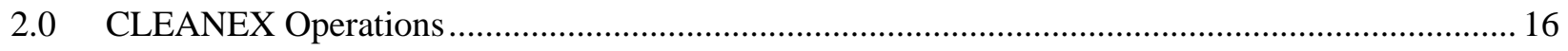

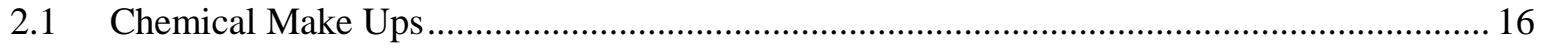

2.2 CLEANEX Feed Adjustment........................................................................................ 18

2.3 CLEANEX Extraction, Scrubbing, Stripping, and Organic Cleanout Operations................. 19

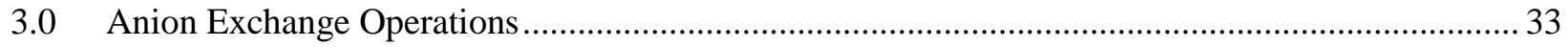

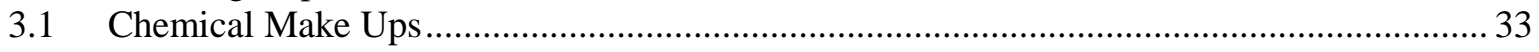

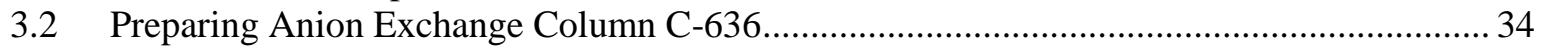

3.3 Pretreatment and Filtration of Feed (Product) Solution .................................................... 36

3.4 Anion Exchange Equipment Set Up, Check Out, and Operations ...................................... 38

3.5 Purification of Transcurium Actinides by HYDROXIDE Precipitation............................... 46

3.6 Disposition of Actinide Product (Cm Product Cuts) from RR-6 Tanks............................... 47

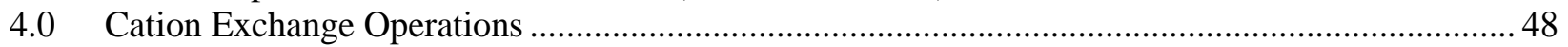

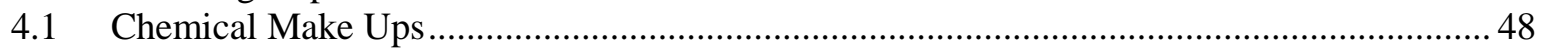

4.2 Loading Column C-402 Resin Removal and Resin Addition ............................................. 49

4.3 Elution Column C-401 Resin Removal and Resin Addition.................................................. 51

4.4 Actinide Loading onto the Loading Column C-402 ......................................................... 53

4.5 Elution and Separation of Actinides Using the Elution Column C-401................................ 54

4.6 Acidification and Sampling of Product Cuts and Raffinate Solutions ..................................56

4.7 Cation Exchange Operation Follow Up and Product Compositing .................................... 56

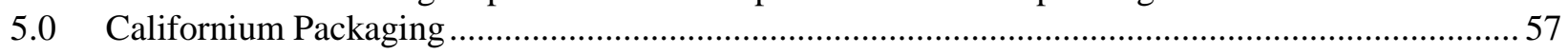

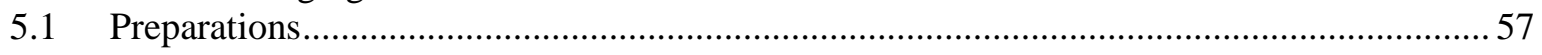

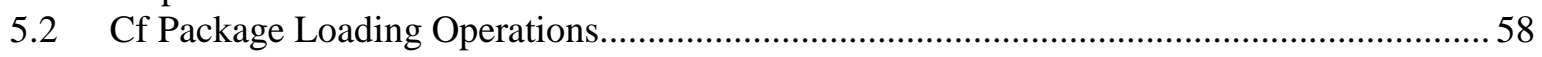

5.3 Cf Package Firing in the Cubicle 4 Muffle Furnace ......................................................... 59

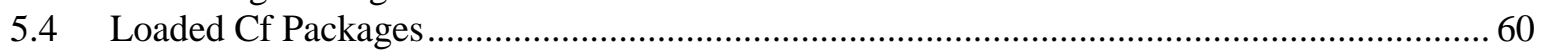

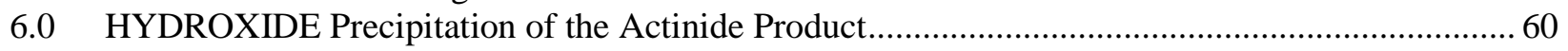

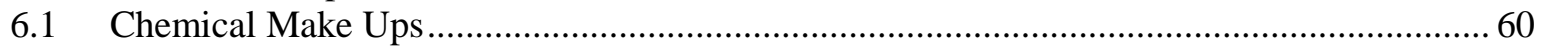

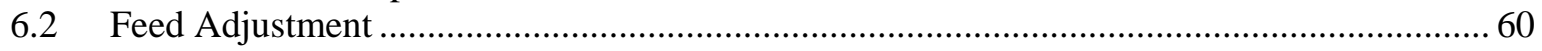

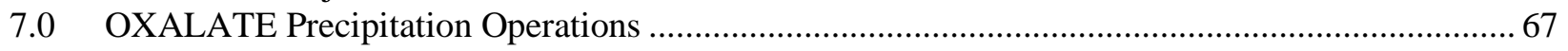

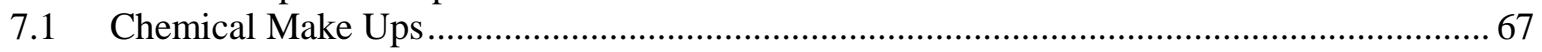

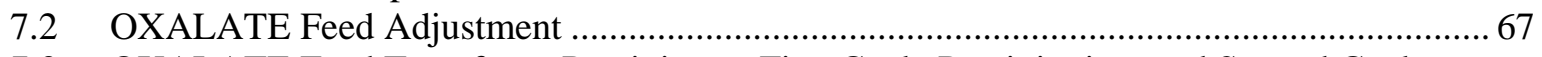

7.3 OXALATE Feed Transfer to Precipitator, First Cycle Precipitation, and Second Cycle Precipitation Operations..................................................................................................... 71

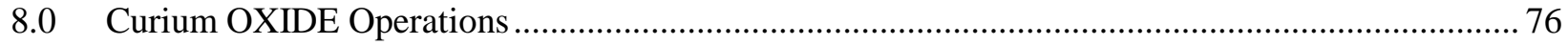

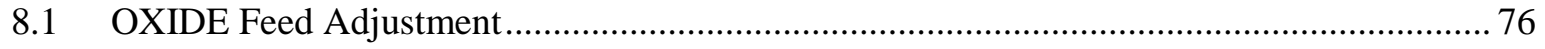

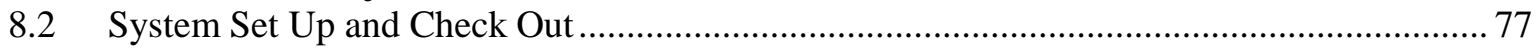




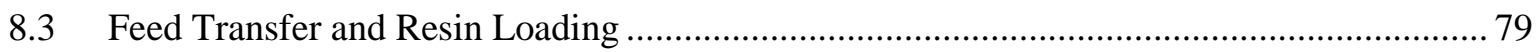

8.4 Resin Burning and Calcination of Actinide Oxide............................................................. 81

8.5 Characterization, Sampling and Storage of an Actinide Oxide Product ................................ 82

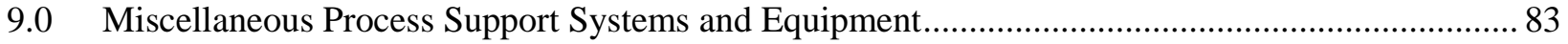

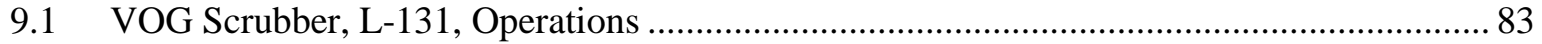

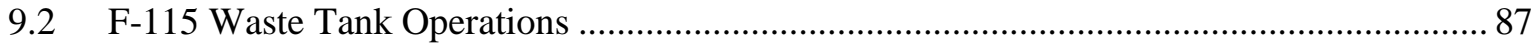

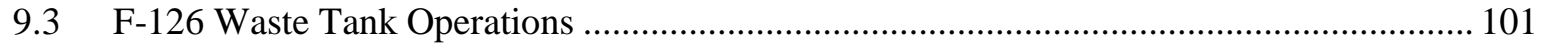

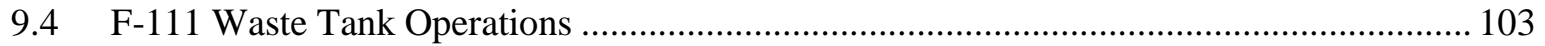

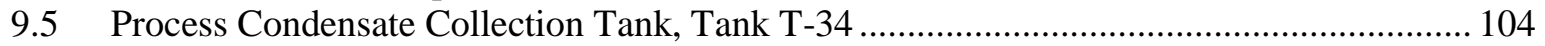

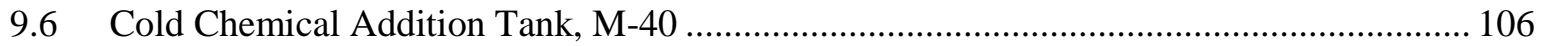

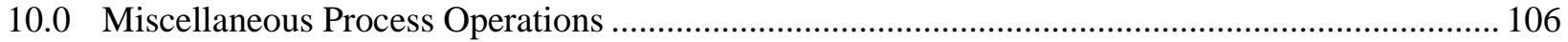

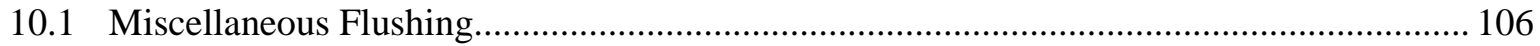

10.2 Miscellaneous Solution Collection Tanks, Tank T-66 and Tank T-79 ............................ 108

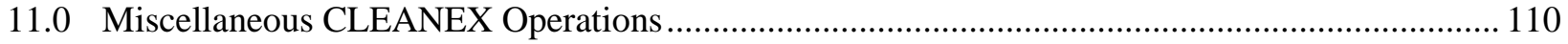

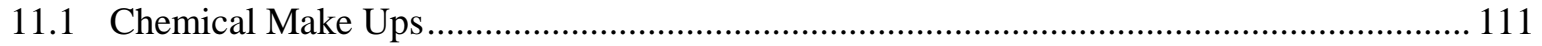

11.2 Miscellaneous CLEANEX extraction, scrubbing, stripping, and organic cleanout

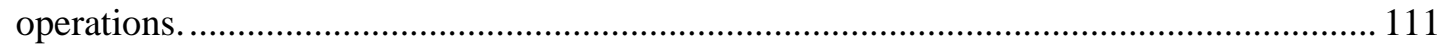

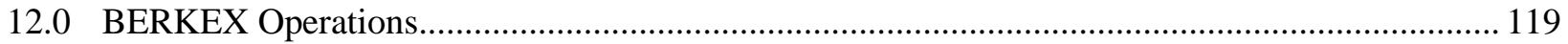

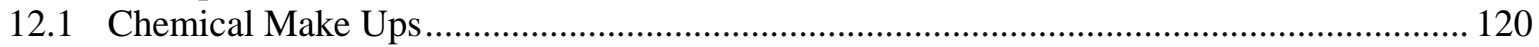

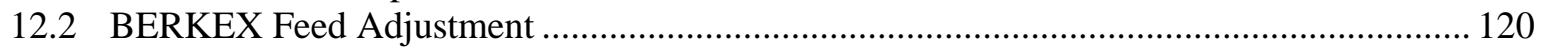

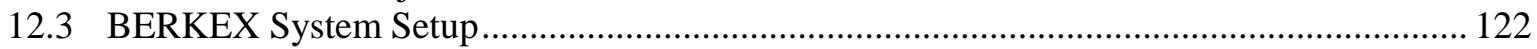

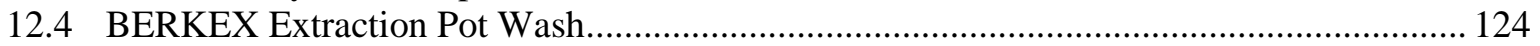

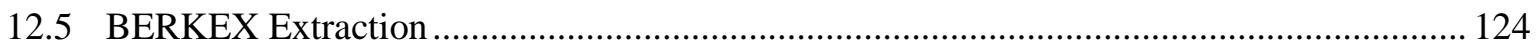

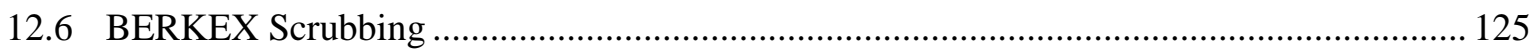

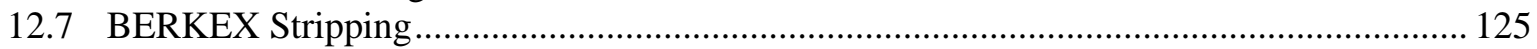

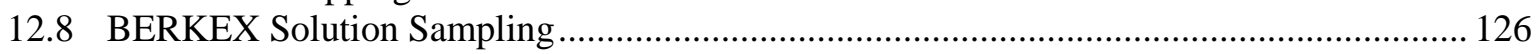

12.9 BERKEX Extraction Pot (T-411) and Vacuum KOP (T-409) Flush................................. 126

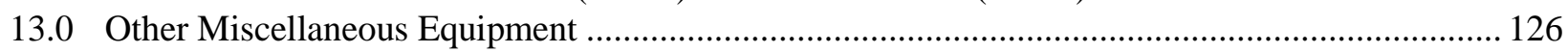

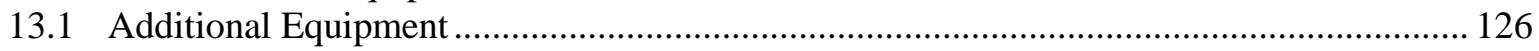

13.2 Equipment Exclusions from Cf Production Program..................................................... 128

APPENDIX A. EQUIPMENT EVALUATION TABLE .................................................................. 


\section{LIST OF FIGURES}

Figure 1. Cut-away view of Building 7920 hot cell cubicle, tank pit, cubicle pit, chemical MUA, Control Room, and LAA.

Figure 2. TRU disconnect base and swing clamp.................................................................................... 2

Figure 3. Typical HFIR irradiated Cm target processing flow sheet. ......................................................... 4 



\section{ACRONYMS}

$\begin{array}{ll}\text { AHIB } & \text { alpha-hydroxyisobuteric acid } \\ \text { BR } & \text { back rack } \\ \text { CU4AJ } & \text { Cubicle } 4 \text { air jet } \\ \text { DC } & \text { disconnect } \\ \text { DP } & \text { differential pressure } \\ \text { FCV } & \text { flow control valve } \\ \text { FDV } & \text { flow diversion valve } \\ \text { GN } & \text { gooseneck } \\ \text { HC } & \text { Hastelloy C } \\ \text { HCl } & \text { hydrochloric acid } \\ \text { HCV } & \text { hand controlled valve } \\ \text { HDEHP } & \text { di-(2-ethylhexyl) phosphoric acid } \\ \text { HFIR } & \text { High Flux Isotope Reactor } \\ \text { HNO } & \text { nitric acid } \\ \text { HV } & \text { hand valve } \\ \text { ID } & \text { inner diameter } \\ \text { KP } & \text { knock out pot designator } \\ \text { KOP } & \text { knock out pot } \\ \text { LAA } & \text { limited access area } \\ \text { LGWO } & \text { Liquid and Gaseous Waste Operations } \\ \text { MB } & \text { Master Panel Board } \\ \text { MCS } & \text { Monitoring and Control Station } \\ \text { MDV } & \text { multiposition diversion valves } \\ \text { MUA } & \text { make up area } \\ \text { OOS } & \text { out of service } \\ \text { PCV } & \text { pressure control valve } \\ \text { PIC } & \text { pressure indicator controller } \\ \text { PS } & \text { phase separator } \\ \text { PVC } & \text { polyvinyl chloride } \\ \text { R\&D } & \text { research and development } \\ \text { RCW } & \text { recirculating cooling water } \\ \text { RE } & \text { radiation element } \\ \text { REDC } & \text { Radiochemical Engineering Development Center } \\ \text { RR } & \text { rear rack } \\ \text { 7PDV } & \text { seven position diversion valves } \\ \text { TRU } & \text { transuranium } \\ \text { UV } & \text { ultraviolet } \\ \text { VOG } & \text { vessel off gas } \\ \text { Zr } & \text { Zircaloy } \\ & \end{array}$




\section{BACKGROUND}

Building 7920 is one of two Category 2 nuclear facilities that comprise the Radiochemical Engineering Development Center (REDC). Building 7920 basic features are nine heavily shielded hot cells, a transfer cubicle with a lazy Susan glove box, two analytical laboratories, four radiological glove box laboratories, one "mini" hot cell laboratory with two "mini" hot cells, two cold chemical laboratories, one chemical make up area (MUA), and a limited access area (LAA). Building 7920, originally referred to as the Transuranium (TRU) Processing Facility, was constructed in 1966 as part of the Heavy Element Research Program for the Atomic Energy Commission. Building 7920's function was to prepare materials and fabricate targets containing ${ }^{244} \mathrm{Cm}$ that were inserted into the High Flux Isotope Reactor (HFIR) for irradiation to transmutate the ${ }^{244} \mathrm{Cm}$ into a number of heavy elements (Es, Fm, Cf, and Bk). The target fabrication work is done in Cells 1, 2, and 3. Once the irradiation process was complete in the HFIR, the irradiated targets were received back into Building 7920 to process and separate these heavy elements for research and development (R\&D). This was done in Cells 4, 6, and 7. Cells 8 and 9 were used by analytical chemistry personnel to prepare and analyze samples. Later Cell 9 was used to manage the solid waste produced in the hot cell bank.

The original life span of the project was approximately 10 years. At present (47 years later), the HFIR and Building 7920 are still producing and processing heavy elements under the purview of the Department of Energy, but the main focus is now the production and recovery of ${ }^{252} \mathrm{Cf}$ and occasionally ${ }^{249} \mathrm{Bk}$. The Department of Energy sells the ${ }^{252} \mathrm{Cf}$ to private industry, where it is sold throughout the world for many diverse uses. The ${ }^{249} \mathrm{Bk}$ has been used as a target material to produce heavy element 117.

Building 7920 has a unique design to be able to deal with the radiation and contamination associated with the heavy elements processed. Also, almost all the equipment was designed and installed for remote maintenance. Each hot cell consists of a cubicle, a tank pit, and cubicle pit (Figure 1). The cubicles are equipped with massive shielded viewing windows, equipment racks, and master slave manipulators to operate the equipment racks. The tank pits contain process tanks and lines interconnecting them to the various other tanks and equipment in the cubicles. The cubicle pit houses the intercell conveyor used for transporting items between the cubicles. The equipment racks in the hot cell cubicles and the tanks in the tank pits use specially designed connectors (TRU disconnects) that allow a pseudo "plug-n-play" capability (Figure 2). In the early years of the program, equipment racks in the cubicles and tanks were changed out as needed to support the R\&D efforts for the program. As the original radiochemical processing $R \& D$ activities became standardized for the separation of the heavy elements, the need for changing out the racks and tanks diminished and most of the equipment racks and tanks have been in use for more than 35 years. 


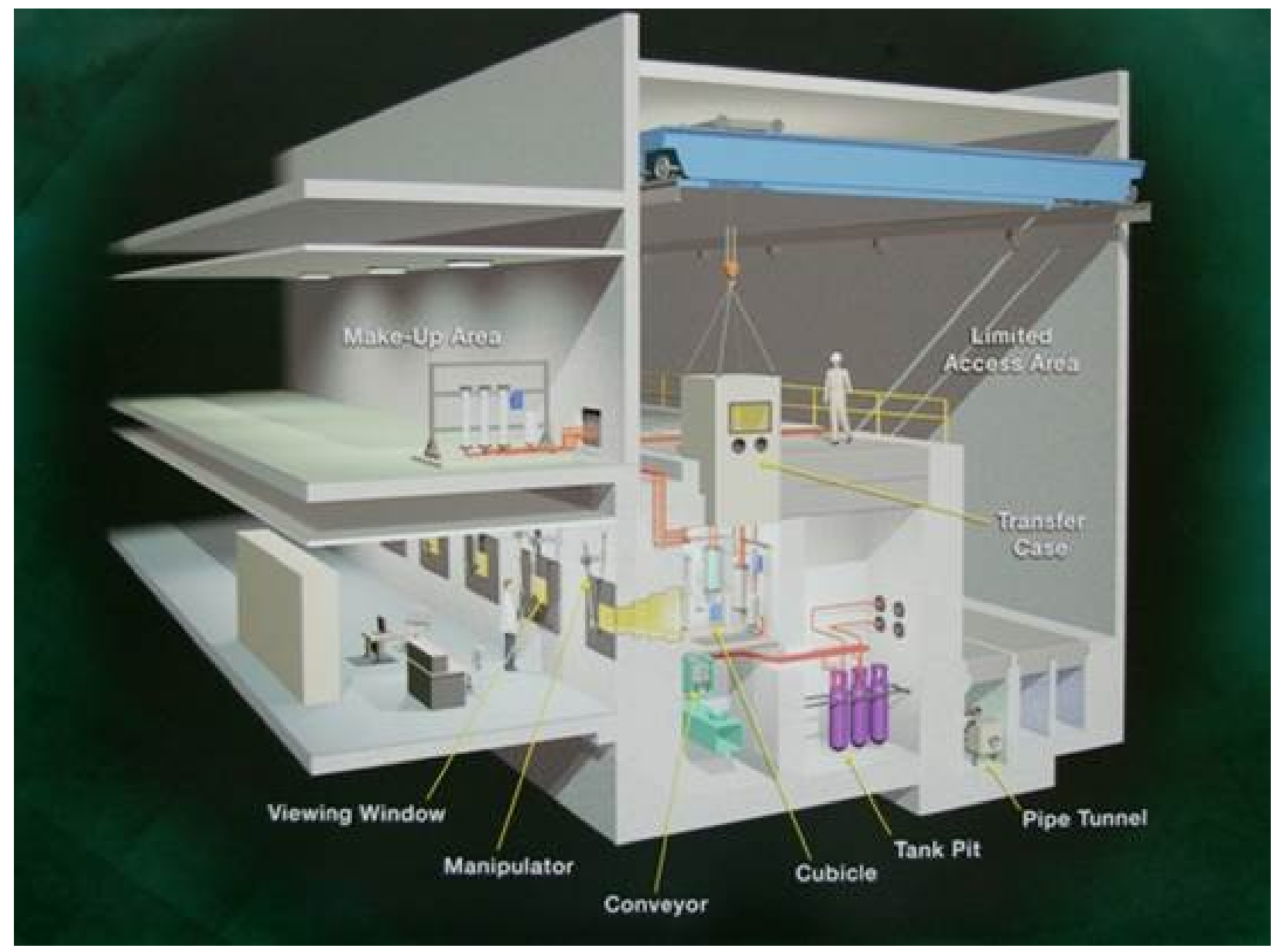

Figure 1. Cut-away view of Building 7920 hot cell cubicle, tank pit, cubicle pit, chemical MUA, Control Room, and LAA. 

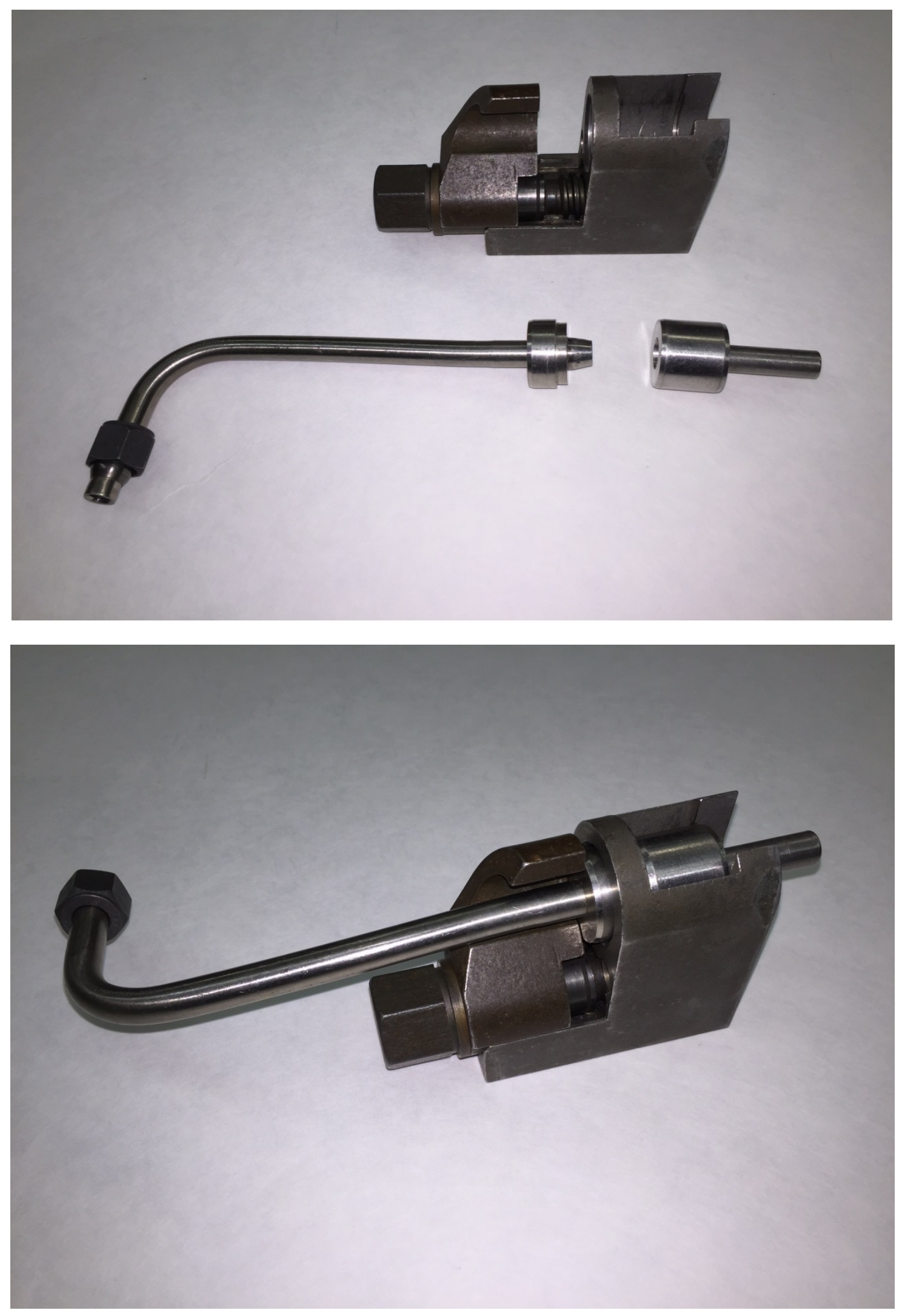

Figure 2. TRU disconnect base and swing clamp. 


\section{TASK OBJECTIVE}

To successfully continue the ${ }^{252} \mathrm{Cf}$ production and meet the needs of the customers, a comprehensive evaluation of the Building 7920 processing equipment was requested to identify equipment critical to the operational continuity of the program.

\section{EVALUATION METHODOLOGY}

To provide a complete evaluation, it was necessary to identify the equipment utilized by each processing operation from dissolution of the irradiated targets rods to the Cf packaging and curium oxide production including miscellaneous material recovery operations and liquid waste management operations. Figure 3 is a typical flowsheet for the process operations that comprise a HFIR ${ }^{244} \mathrm{Cm}$ Target Processing Campaign. Each piece of equipment utilized for these process operations was evaluated for potential failure, impact of failure, spare parts availability, and estimation of repair time. Many pieces of equipment are utilized multiple times within a set of operations. For example, during CLEANEX stripping operations, multiple strips are added and transferred to a product collection tank. A vacuum/pressure transfer system and a pump transfer system are used to transfer each strip. Also, many components of these same transfer systems are used for other process operations as well. The individual pieces of equipment that comprises these systems are listed for each time they are used to emphasize the importance of each piece of equipment and system to the overall operational success.

Spare parts are typically available for many of the common and widely used pieces of equipment, such as flow diversion valves (FDV), polyvinyl chloride (PVC) toggle hand valves for hand-controlled valve (HCV) positions, seven position diversion valves (7PDV), and multiposition diversion valves (MDV), to name a few. These are generally fabricated in house or subcontracted to an outside supplier and mass produced. For simplification purposes, the "fabricated in house" references in the Equipment Identification and Evaluation section are meant to imply that these components are fabricated either in house or by an outside supplier. Even though failure of one of these components can shut down operations, repairs can be performed quickly, and the shutdown is very brief. These types of equipment failures are not considered as critical.

There are other pieces of equipment that no spare inventory is maintained. This can be the result of prior insufficient funding levels, the relatively high cost for certain replacement parts, replacements that are no longer available, or a lack of manpower to procure comparable spares. For certain equipment with no spare inventory, procuring a replacement, though somewhat expensive, is relatively quick and replacing the failed equipment is easy and does not have a significant impact on the overall operational success. But for other equipment, there may be a long lead time to procure the equipment, or replacing the failed equipment is difficult. Ultimately, the overall operational success is negatively impacted. For these types of equipment failures, it was considered to be a critical piece of equipment.

Consideration was also given to trends for equipment failure. Because the facility has been in operation for 47 years, there have been noted trends for certain equipment failures. Also, certain other crucial equipment has been replaced at a prescribed time interval to avoid potential catastrophic failure. Typically, this type of equipment has shown a trend of decreased functionality after a certain period of time, which has served as an indicator for impending failure.

Based on these trends and operational experience, the equipment was evaluated to determine where potential equipment failures could exist. The write up in the Equipment Identification and Evaluation section divides a processing campaign into the unit operations required to accomplish the entire processing campaign. Each unit operation is briefly described to present how unit operation flows and how the equipment is utilized. Each piece of equipment is listed with information about availability of 
spares and estimation of repair/replacement duration. Equipment that is more complex in nature is given a more comprehensive discussion. When a particular piece of equipment is used more than once, it is referenced back to the section number where it was first discussed.

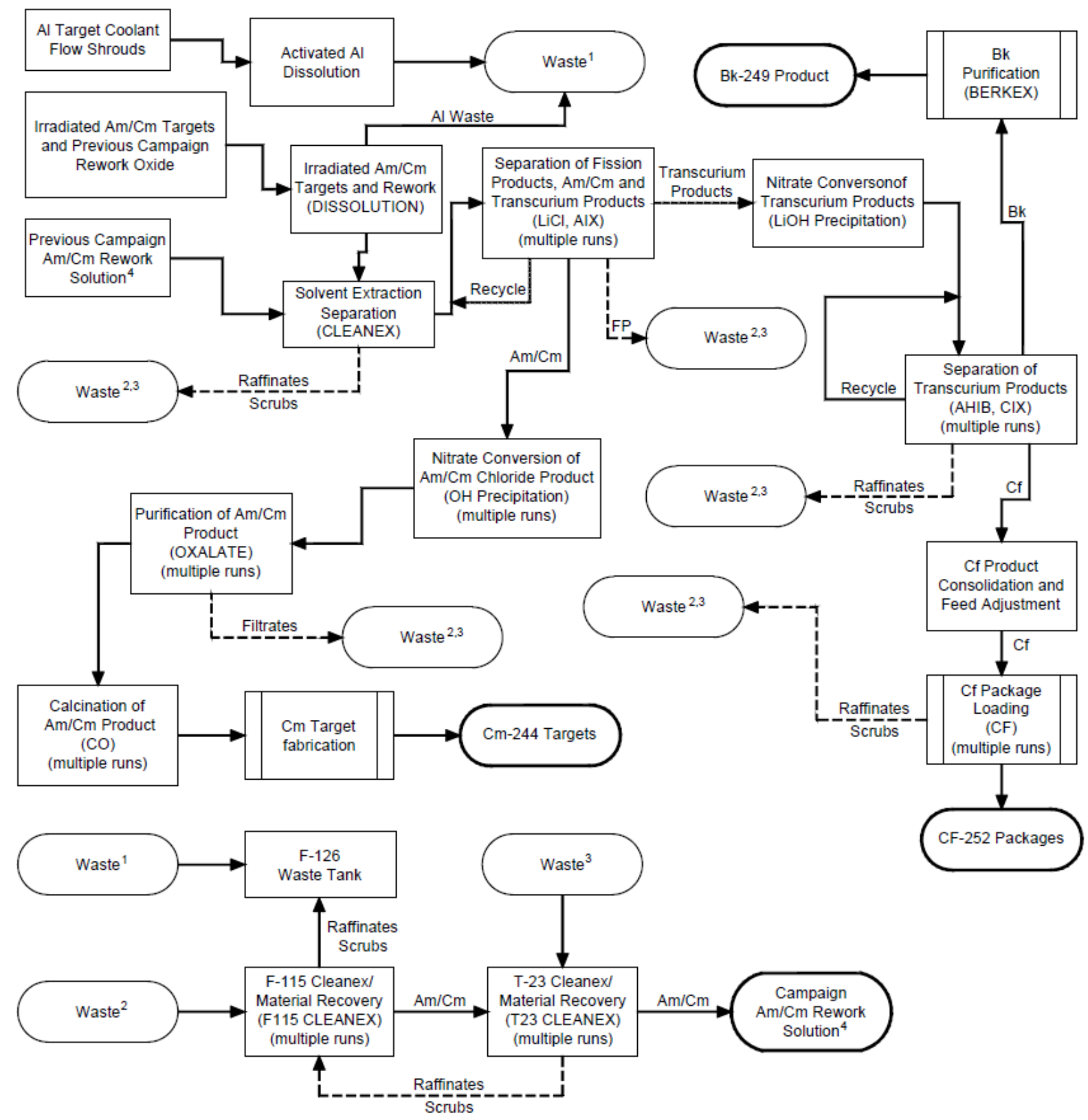

Figure 3. Typical HFIR irradiated Cm target processing flow sheet.

Appendix A, Equipment Evaluation Table, lists each piece of equipment, gives a brief description of the equipment, gives the reference section where it can be found in the Equipment Identification and Evaluation section, and rates how critical it is to overall operational success. The legend for the Equipment Evaluation Table describes how the rating system is applied to the equipment. 


\section{EQUIPMENT IDENTIFICATION AND EVALUATION}

\subsection{Target Rod Dissolution (Including Target Rod Hex Can Removal and Scrap Al Dissolution)}

\subsection{Target Rod Hex Can Removal}

The target rods are received into Cubicle 7 from the 25-T Savannah River Plant shielded carrier in a handling "spider." The target rods have the hex cans (cooling shrouds) still attached, and the hex cans must be removed before dissolution of the target rods.

1.1.1 Slitter rail and cutter assembly-Used to remove the hex cans from the target rods. The slitter rail is very robust and has not been replaced in over 40 years of service. The cutter assembly fits onto the slitter rail and has metal bearings that act as wheels for the assembly to slide the length of the slitter rail. The assembly has a standard cutter wheel from a tubing cutter, and the depth that the cutter wheel cuts can be adjusted by turning a knob on top of the assembly. The cutter wheel used in the cutter assembly has to be replaced periodically, but this is not too difficult to accomplish. The housing for the cuter wheel was replaced in February 2017 and is typically replaced on an infrequent basis. A spare housing is not available; therefore, a spare housing would have to be fabricated, if needed.

1.1.2 Modified hand tools-Modified for in-cell use, a modified screwdriver, one of the most common tools used in the hot cell cubicles, is used to aid in hex can removal. Spares are readily available. Other miscellaneous modified tools may be utilized to facilitate the removal of the hex cans from the target rods. Most of these tools are readily available for use.

\subsection{Chemical Make Ups}

1.2.1 M-1 - A chemical make up/storage tank used for the $\mathrm{NaNO}_{3}$ make up. M-1 originally was a Penton lined and coated tank and was recently replaced with a newly fabricated (in house) PVC tank with Hayward PVC ball valves on all the PVC inlet and outlet lines. M-1 is a nominal $50 \mathrm{~L}$ volume tank. The tank itself should last many years with no required maintenance. The ball valves sometimes develop leaks, but typically the valves are very reliable. Normally, leaking valves can be repaired, but replacement is usually about as cost-effective as repairing. The lines and valves associated with the tank are listed below:

a. V-M1-1, vent line to vessel off gas (VOG)

b. V-M1-2, solution addition line through the funnel

c. V-M1-3, 5 psig air pressure supply line

d. V-M1-4, outlet line/drain

e. V-M1-6, solution addition line

f. V-M1-7, outlet line

g. V-M1-11, sight glass valve at bottom of sight glass

h. V-M1-13, outlet line 
1.2.2 M-2-A chemical make up/storage tank used for $\mathrm{NaNO}_{3}$ make up. $\mathrm{M}-2$ originally was a Penton lined and coated tank and was recently replaced with a newly in house, fabricated PVC tank with Hayward PVC ball valves on all the PVC inlet and outlet lines. M-2 is a nominal $50 \mathrm{~L}$ volume tank. The tank should last many years with no required maintenance. The ball valves sometimes develop leaks, but typically the valves are very reliable. Normally, leaking valves can be repaired, but replacement is usually about as cost-effective as repairing. The lines and valves associated with the tank are listed below:

a. V-M2-1, vent line to VOG

b. V-M2-2, solution addition line through the funnel

c. V-M2-3, 5 psig air pressure supply line

d. V-M2-4, outlet line/drain

e. V-M2-6, solution addition line

f. V-M2-7, outlet line

g. V-M2-11, sight glass valve at bottom of sight glass

h. V-M2-13, outlet line

\subsection{Target Rod Dissolution and Solution Transfers}

\subsubsection{Dissolver Tank, T-70}

a. T-70 - A 171 L capacity tank constructed of Zircaloy (Zr) that is located just beneath the floor in Cubicle 7. T-70 has a 4.75 in. diameter access port at the top of the tank nearly in the center of Cubicle 7 floor. It consists of an Oring sealed lid that is positioned in place by two ears located $180^{\circ}$ apart. The lid is tightened by turning a bolt on the top of the lid, which pushes the lid down to seal the O-ring in the lid to the tank top. The irradiated HFIR curium targets are charged to T-70 through this access. The O-ring seal has been replaced many times. The O-rings are readily available, and replacement is accomplished in a matter of hours.

b. Foxboro pneumatic instrument-T-70 liquid level, density, and pressure is read out and recorded on an original Foxboro pneumatic instrument (over 40 years old). This instrument is typically reliable and is routinely calibrated. Broken polyethylene lines supplying the pneumatic signals to the transmitters and instrument can present problems, but in recent years, UV-resistant polyethylene has decreased potential breaking of lines because of age and exposure to UV light. Plugged lines can be a problem if the plug cannot be removed easily.

c. T-70 spargers-An electric switch activates a solenoid that opens and allows air to flow into a "deep leg" in the tank for solution mixing in the tank. There are two spargers for T-70, which are selected by a selector switch. One provides a low flow rate of air that is regulated by an orifice plate in the sparge line. The other provides a high flow rate of air and is manually regulated by a flow meter. The orifice plate can plug requiring the suspension 
of sparging operations in T-70. The orifice plate would have to be removed and cleaned. This typically requires less than $4 \mathrm{~h}$. Plugs in the sparge line downstream of the orifice or flow meter can occasionally occur. This can sometimes require as many as $12-24 \mathrm{~h}$ to clear. The sparger solenoid has failed, even though failure is infrequent. Rebuilds and replacements are readily available and cause very minimal down time for repairs.

d. T-70 processing lines-Constructed of $\mathrm{Zr}$, these are the lines that are used to transfer process solutions into and out of T-70. Typically, these lines are not an issue, though at times some lines are preferred over others, and plugged lines can be a problem if the plug cannot be removed easily or an alternate line cannot be used. Also, because T-70 is accessible from Cubicle 7 floor, temporary lines for transfers can be made up and lowered into T-70 using the Cubicle 7 master slave manipulators.

e. T-70 thermocouple-This is a specially fabricated thermocouple because of its longer length. The thermocouple is replaced in T-70 in Cubicle 7 using the master-slave manipulators. This thermocouple experiences more frequent failure rate than most of the other thermocouples associated with the hot cell operations due to its connector being exposed to the working area in Cubicle 7. The lead time for procuring this thermocouple is approximately 23 weeks. Installation in the thermo-well in T-70 is tedious. The thermocouple wire is about $0.0625 \mathrm{in}$. in diameter, and the entire length of the thermocouple has to be inserted into the thermo-well. Installation requires about $4 \mathrm{~h}$.

\subsubsection{P-78 Heating Loop}

a. P-78 pump-This pump is located in the MUA and was replaced in 2014. It had been very reliable, but its age was greater than 40 years old and the only available replacement was used. An equivalent replacement pump has not been identified at this time. A comparable pump could be scavenged from one of the other heating loops if necessary to avoid a significant time delay, but that would have to be determined to be cost-effective because most of the other possible replacement pumps are very old as well and may not have the same flow capabilities. The time, cost, and effort may not warrant using a potentially unreliable pump in this situation. It would be better to procure an equivalent replacement. Procuring and installing an acceptable replacement might require a significant amount of time (as much as $2-3$ weeks or even longer).

b. V-M74-1, V-M74-2, V-M74-3, V-M74-10, V-M74-12, V-T555-2, V-T5553, V-T555-15, V-H78-1, V-H78-2, V-T70-2, V-T70-3, V-T70-9, V-T70-10, V-T70-11, V-T70-15, and PRV-P78-5-Part of the P-78 heating loop system. All the valves appear to be functional, with exception of one valve that is stuck in the open position. All these valves, but one, are a combination of gate and ball valves from various manufacturers, and equivalent replacements for these valves are kept on hand. Typically, these do not 
experience instantaneous complete failure. They usually start leaking, and the leak gets worse to the point that it is necessary to change the valve. The one valve that is not a ball or gate valve is a pressure relief valve. Valve change out would typically be a $2-4 \mathrm{~h}$ job, depending on whether or not the loop was being used just prior to the change out and had to cool down before being worked on.

c. Tank M-74-Located on Tank Island 7 in the MUA, this tank is stainless steel and nearly maintenance free. There have been no problems with its functionality in more than 40 years of service. Failure of a magnitude that would make it nonfunctional is very unlikely. If a gross failure occurred, a new one would have to be specified and fabricated because it is not an off the shelf item. This could require 3 or more weeks.

d. H-78A heat exchanger and H-78B heater-Located on Tank Island 7 in the MUA, these components have not been replaced in over 40 years. They have been reliable, but their age could be a factor. Procuring a replacement heater for H-78B has been explored, and a "like kind" replacement heater is available. The procurement lead time would be approximately 4 weeks. If necessary, the heater (H-763B) from the P-761/H-763 heating loop system might be scavenged to restore $\mathrm{H}-78 \mathrm{~B}$ to avoid a significant time delay for procuring a new heater. The H-78A Heat Exchanger would be significantly easy to replace if failure occurred because it is a relatively simple heat exchanger.

e. TRC-T70-Located in the Control Room, this is the temperature indicator/recorder controller for the H-78/P-78 heating loop. This controller was recently replaced (May 2014). The previous controller had been in service for over 40 years with little or no problems experienced in that time.

f. TCV-T70 - Located on Tank Island 7 in the MUA, this is a temperature control valve (Research Control Valve) manufactured by Badger Meter used to regulate the amount of cooling water introduced into the P-78 loop. This valve was replaced in 2015. The previous valve was a Research Control Valve from Badger Meter also that had been in operation for over 40 years, and it functioned satisfactorily until 2015. An identical replacement has been procured as a spare.

g. FI-T70-2 and FI-T70-3-Located on Tank Island 7 in the MUA, these flow indicators have been reliable. They have required very little or no maintenance for over 40 years of service. It is unlikely that a total failure of these flow indicators would occur. Currently, there are no spares on hand, but acceptable replacements could be procured and installed relatively quickly.

h. H-78 thermocouples_Located on Tank Island 7 in the MUA, these are off the shelf Type K thermocouples. Failure of any of these thermocouples can be instantaneous with no warning, or it can be a gradual failure with some indication that the thermocouple is about to fail. These thermocouples are critical for performing the dissolution of target rods in T-70. 
1.3.3 M-573-A nominal 9.5 L volume glass column (4 in. diameter $\times 48$ in. tall) tank. M-573 is located in the MUA and is used to meter $\mathrm{NaOH}$ into T-70 for dissolution of $\mathrm{Al}$ target rods. The tank itself should last many years with no required maintenance. All the inlet and outlet lines have Jamesbury ball valves. The ball valves occasionally develop leaks, but typically they are very reliable. Normally, leaking valves can be repaired, but replacement is usually about as cost-effective as repairing. The FC-M573 system has a flow controller, FICM573, that operates a flow control valve (FCV) based on flow rate measurements from an in-line differential pressure cell, FT-M573. The flow control systems are fairly old with some potential for failure. This could delay operations up to $24 \mathrm{~h}$ for repairs or to prepare other systems to perform this function. The lines and valves associated with the tank are listed below:
a. V-M573-1, vent line to VOG
b. V-M573-2, solution addition line through the funnel
c. V-M573-3, 5 psig air pressure supply line
d. V-M573-4, drain line
e. V-M573-6, 25 psig air pressure supply line
f. V-M573-7, outlet to FCV-M573
g. V-M573-9, discharge from FCV to LR-5
h. V-M573-10, FCV bypass
i. V-M573-11, outlet line from FCV
j. V-M573-12, outlet line from FCV
k. V-M573-13, sample line
l. FCV-M573, flow control valve
m. FT-M573, differential pressure transmitter
n. FIC-M573, flow indicator/controller for FCV-M573

\subsection{Al Dejacket Solution Transfer}

1.4.1 The transfer route is from T-70 to F-111 using T-737 vacuum/pressure tank on back rack (BR)-7 to perform the transfer. Current route is: T-70 $\rightarrow$ T-70

(Nozzle L) $\rightarrow$ T7009 gooseneck (GN) $\rightarrow$ polyethylene line to T7014 GN (BR-7) $\rightarrow$ 7PDV-T70-1 (Type B set to 5) $\rightarrow$ F-70 $\rightarrow$ special FDV at FDV-F70 position with line at left $\rightarrow$ polyethylene line $\rightarrow$ PVC ball valve at P72010 GN $\rightarrow$ 7PDVP720-1 (Type D set to 2) $\rightarrow$ T73702 $\rightarrow$ T-737 $\rightarrow$ 1-line block at hand valve (HV) HV-T731-1 with line at top $\rightarrow$ PVC ball valve $\rightarrow$ polyethylene line to 1-line block at F-111 sampler with line at left $\rightarrow$ F-111.

Cell equipment utilized:

a. T7009 GN on T-70 dissolver in Cubicle 7 floor-Standard Zr GN with polyethylene line connected to go to T-7014 GN on BR-7; fabricated in house. Typically spares are available. 
b. T7014 GN on BR-7-Standard Zr GN with polyethylene line connected to come from T-7009 GN on T-70; fabricated in house. Typically spares are available.

c. 7PDV-T70-1 (Type B set to 5) on BR-7-Fabricated in house. Typically spares are available.

d. F-70 filter on BR-7-Specially fabricated sintered metal stainless steel filter that is fabricated in house. One spare filter is available in the South Storage Area.

e. Special FDV at FDV-F70 position (line at left) on BR-7-Used with a polyethylene line connected to go to P72010 GN; fabricated in house. Typically spares are available.

f. P72010 GN on BR-7-Standard Zr GN with polyethylene line and PVC ball valve (Haywood pressure control valve [PCV] ball valves equipped with a JACO fitting on each end for use in the hot cell cubicles) connected to come from special FDV at FDV-F70; fabricated in house. Typically spares are available.

g. 7PDV-P720-1 (Type D set to 2) on BR-7-Seven position diversion valve that is fabricated in house. Typically spares are available.

h. Tank T-737 on BR-7-A nominal 7.5 L Ta tank integral to BR-7. A leak in the tank or its lines going to or from the BR-7 connections would render it useless, no way to replace tank without extensive effort.

i. 1-line block at HV-T737-1 position (line at top) on BR-7-With PVC ball valve and polyethylene line installed connected to 1-line block at SA-F111 position; fabricated in house. Typically spares are available.

j. 1-line block at SA-F111 (F11102 line to F-111) on BR-7-With polyethylene line connected to 1-line block at HV-T737-1 position; fabricated in house. Typically spares are available.

k. V-P70-3 in the MUA-Haywood PVC ball valve; typically spares are available.

1. HCV-P70-1 on BR-7-PVC block toggle hand valve installed (could substitute a special 2-line polyethylene block with a PVC valve and polyethylene line connecting the two lines); fabricated in house. Typically spares are available.

m. FDV-P70-1 (set top to right) on BR-7-Fabricated in house. Typically spares are available.

n. F-111 waste tank in the waste tank pit-Receives the Al dejacket waste. Refer to Section 9.4 for discussion of F-111 waste tank.

o. KP-738 on BR-7-A knock out pot (KOP) for the T-737 vacuum/pressure transfer system. Gaskets, O-rings, and glass tubes are typically available for rebuilding. Spare base and top plates may not be available, but these normally do not require replacement.

p. CU7AJ-In-cell air jet used for vacuum source. Typically spares are available. 
q. Polyethylene tubing-For vacuum lines, pressure lines, demineralized water lines, and portions of the transfer route. Male ball joints, and plastic compression fittings are readily available.

1.4.2 M-704-A nominal 5.5 L volume glass column (3 in. diameter $\times 48$ in. tall) tank. M-704 is located in the MUA and is used for solution additions to T-70 supporting the dissolver operations. Inlet lines into M-704 come in through the top flange, and there is one outlet line exiting through the bottom flange. The inlet lines and outlet line have Hayward PVC ball valves for air pressure, water and venting utilities, solution addition, and solution discharge. Typically, this type tank requires minimal maintenance, and spare parts are readily available if required. The ball valves sometimes develop leaks, but typically they are very reliable. Normally, leaking valves can be repaired, but replacement is usually about as cost-effective as repairing. The lines and valves associated with these tanks are listed below:
a. V-M704-1, vent line to VOG
b. V-M704-2, solution addition line through the funnel
c. V-M704-3, 5 psig air pressure supply line
d. V-M704-4, drain line/solution outlet
e. V-M704-7, demineralized water addition line

\subsection{Dissolution of Actinide Oxides}

NOTE: All the pieces of equipment identified in Sections 1.5.1 and 1.5.2 below were already discussed in Sections 1.4.1 and 1.3.2, respectively, because they are used for the target rod dissolution and Al dejacket solution transfer.

1.5.1 P-70 back flush route- The transfer route for solution additions into T-70 utilizes the P-70 back flush route. M-704 is typically used to make these additions. Refer to Sections 1.4.1 and 1.4.2 for a discussion of M-704 and the portion of the addition route used for solution additions.

1.5.2 P-78 heating loop-Utilized to dissolve the actinide oxides in T-70. Refer to Section 1.3.2 for a discussion of the P-78 heating loop.

1.5.3 T-70 sampler (vacuum accumulator and needle block) - If the T-70 sampler is used, then the vacuum accumulator may periodically need to be rebuilt with a new glass, gaskets, and O-ring. (This is the preferred method.) These spare parts for the vacuum accumulator are typically available. A functional needle block assembly is also required to use the T-70 sampler. Needle block assembly components have typically been fabricated in house, but recently they have been fabricated by an outside machine shop. Needle block assemblies can be rebuilt in the hot cell cubicles with new needles, O-rings, and packing nuts, if spare parts are available. If spare parts are not available, 4 weeks or more lead time may be required to have these parts fabricated. The needle blocks themselves require a 
minimum 4 weeks lead time for fabrication. In recent years, maintaining an ample supply of functioning needle block assemblies has been difficult, and a $1 \mathrm{~L}$ glass bottle with a 2-line rubber stopper configured for sampling and connected to T-70 sample uptake line with polyethylene tubing has been used for sampling. Glass bottles, stoppers, polyethylene tubing with female ball joints, polyethylene tubing, and plastic compression fittings are kept in stock and typically readily available.

\subsection{Actinide Product Solution Transfer from T-70 to T-72}

1.6.1 The actinide product solution transfer from T-70 to T-72 can be performed using several routes. This makes equipment failure that could potentially cause significant delays in operations very unlikely because an alternate transfer route and system can be utilized. Two of the most common transfer routes that are the most likely to be used are detailed below.

\section{T-734 vacuum/pressure tank (on RR-7) to perform the transfer:}

Cell equipment utilized:

a. T-70 Nozzle L/gooseneck (GN) at top of T-70 in Cubicle 7 floor-Used with a polyethylene line connected to the inlet of F-720. There are three deep legs in T-70 that could potentially be used to transfer solution out of T-70.

b. F-720 (setup in floor base/holder) - This filter is larger than the filters routinely used in the hot cell cubicles. It is typically rebuilt with a 3 in. diameter $\times 10$ in. long glass pipe, flat gaskets, O-ring, and specially modified filter element. The filter elements are procured as standard $10 \mathrm{in}$. long filter elements but have to have a small amount cut off the end to fit into the special F-720 filter housing. Typically, there are spare glasses, gaskets, Orings, and modified filter elements available. F-720 is typically a reliable piece of equipment that is fabricated in house and has been replaced once in the last 35 years. Presently, there are no spare $\mathrm{Zr}$ bottom plates or top plates available should F-720 need to be replaced.

c. Floor base/holder for F-720 — Specially fabricated holder for F-720 to be able to use F-720 in the floor of Cubicle 7. The holder consists of a mounting base with TRU ferrules and a mounting bolt that mimics the BR-7 position for F720 with inlet and outlet lines at the back of the mounting base to connect polyethylene lines to using plastic compression fittings (JACOs). No spare is available.

d. T73402 GN on RR-7-Standard Ta GN with polyethylene line connected to outlet of F-720; fabricated in house. Typically spares are available.

e. Special 2-line polyethylene block at PS-733 position on RR-7-Used with polyethylene tubing connecting both lines on the block; fabricated in house. Typically spares are available.

f. Special 2-line block at HV-T734-2 position on RR-7-Inlet valve position for T-734 tank with a polyethylene line and a PVC ball valve connected the two lines. Spare PVC ball valves and polyethylene line are readily available. 
g. Tank T-734 on RR-7-Cubicle rack tank constructed of Ta metal with a nominal 7.5 L volume. This tank is the newest metal tank in the hot cell cubicles and should need no maintenance.

h. 2-line block at HV-T734-3 position on RR-7-Special polyethylene block with polyethylene tubing connecting both lines on the block; fabricated in house. Typically spares are available.

i. T73404 GN on RR-7-Standard Ta GN outlet from T-734 with polyethylene line connected to 1-line block at SA-T72 position, fabricated in house. Typically spares are available.

j. $\quad$ T7202 GN in Cubicle 7 right disconnect (DC) well (T7202 line to T-72)— Standard Ta GN with polyethylene line connected to T73404 GN; fabricated in house. Typically spares are available.

k. HV-T734-1 position (flush addition line for T-734 transfer system) on RR7-Valve uses a circle seal-style hand valve stem in a metal body. O-rings are available to refurbish leaking valve stems. Also, spare stems can be made available on somewhat short notice. A leaking valve body could present a slightly more significant challenge, but down time would be minimal to repair leaking valve stems or replace a leaking valve, if spare valve bodies are available. A special 2-line block for these type of valve positions with polyethylene tubing and a PVC ball valve connecting the two lines can be substituted as an alternate for the actual valve. Typically these components are readily available.

l. KP-735 on RR-7-A KOP for the T-734 vacuum/pressure transfer system. Typically, gaskets, O-rings, and glass tubes are available for rebuilding. Spare base and top plates may not be available, but these normally do not require replacement.

m. CU7AJ-In-cell air jet used for vacuum source. Previously discussed in Section 1.4.1.p above.

n. Polyethylene tubing-For vacuum lines, pressure lines, demineralized water lines, and portions of the transfer route. Polyethylene tubing, male ball joints, and plastic compression fittings (JACOs) are readily available. Previously discussed in Section 1.4.1.q above.

P-70 vacuum/pressure diaphragm pump (on BR-7) to perform the transfer: Cell equipment utilized:

o. T-70 Nozzle L-Refer to Section 1.6.1.a above.

p. F-720 (set up in floor base/holder)—Also used for this transfer route. Refer to Section 1.6.1.b above.

q. Floor base/holder for F-720_-Also used for this transfer route. Refer to Section 1.6.1.c above.

r. Special 2-line block at F-70 position on BR-7 with polyethylene tubing connected to the right side-Fabricated in house. Typically spares are available. 
s. Special FDV at FDV-F70 position on BR-7 (set top to left)—Previously discussed in Section 1.4.1.e.

t. P-70 vacuum/pressure diaphragm pump on BR-7-Referred to as a TRU pump because it was designed specifically for use in Building 7920; no spares are available. Some spare parts may be available to rebuild/refurbish these pumps, but most, if not all, of the current Hot Cell Operations personnel are inexperienced at rebuilding this type of pump. Failure of the P-70 pump could cause significant down time to repair to return to service.

u. HCV-P70-1 on BR-7-Previously discussed in Section 1.4.1.I above.

v. FDV-P70-1 on BR-7 (set top to right) for back flushing the transfer routePreviously discussed in Section 1.4.1.m above.

w. 7PDV-P730-1 on BR-7 (Type A set to 2)—Fabricated in house. Typically spares are available.

x. MDV-P720-2 on BR-7 (set to any position other than 9)—Fabricated in house. Typically spares are available.

y. 7PDV-P710-2 on BR-7 (Type B set to 1) —Fabricated in house. Typically spares are available.

z. Polyethylene tubing for portions of the transfer route-Polyethylene tubing, male ball joints, and plastic compression fittings (JACOs) are readily available. Previously discussed in Section 1.4.1.q above.

\subsubsection{CLEANEX Feed Tank, Evaporator T-72}

T-72 has several systems that are required to operate properly to maintain functionality. Typically, tank pit tanks like T-72 do not have many physical or mechanical issues that interrupt routine operations. Loss of steam and cooling water capabilities could have a negative impact, but this is a low probability.

a. T-72-A nominal 74 L Hastelloy C (HC) shell with Ta liner evaporator tank located in Tank Pit 7. T-72 has an annulus filled with Hg for increased heat transfer capability and is used to evaporate the dissolved actinide product from the target rods as part of the hot feed adjustment for CLEANEX operations.

b. T-72 instrument lines—Lines in the tank are constructed of Ta. T-72 liquid level, density, and pressure are read out and recorded on an original Foxboro pneumatic instrument (over 40 years old). This instrument is typically reliable and is routinely calibrated. Broken polyethylene lines supplying the pneumatic signals to the transmitters and instrument can present some problems, but in recent years, ultraviolet (UV)-resistant polyethylene has decreased potential breaking of lines due to age and exposure to UV light. Plugged lines can be a problem if the plug cannot be removed easily.

c. T-72 sparger-An electric switch activates a solenoid that opens and allows air to flow into a deep leg in the tank for solution mixing in the tank. The air flow into the tank is regulated by an orifice plate in the sparge line. The orifice plate can plug requiring the suspension of sparging operations in T- 
72. The orifice plate would have to be removed and cleaned. This typically only requires no more than $4 \mathrm{~h}$. Plugs in the sparge line downstream of the orifice or flow meter can occasionally occur. This can sometimes require as many as $12-24$ h to clear. The sparger solenoid has failed, even though failure is not frequent. Rebuilds and replacements are readily available and cause very minimal down time for repairs. If necessary an alternate method of sparging T-72 is by using the in-cell air and applying air pressure down the T-72 sample uptake line.

d. T-72 processing lines-These are the Ta lines that are used to transfer process solutions into and out of T-72. Typically, these lines are not an issue, though at times some lines are preferred over others, and plugged lines can be a problem if the plug cannot be removed easily or an alternate line cannot be used.

e. T-72 sampler-The normal T-72 sampling system has been out of service for many years due to a leaking line in the piping on the sample rack. A $1 \mathrm{~L}$ glass bottle with a 2-line rubber stopper for sampling in Cubicle 7 is connected, using polyethylene tubing, to T7202 GN in Cubicle 7 right DC well. Glass bottles, stoppers, polyethylene tubing with female ball joints, polyethylene tubing, and plastic compression fittings are kept in stock and typically readily available. (Even if there was not a leaking line on the T-72 sample system, the normal sample technique would not be employed because of a lack of functioning needle blocks.)

\subsubsection{T-72 Heating/Cooling}

a. Thermocouples - For monitoring the temperature during evaporation and feed adjustment. The thermocouples come off the tank in the tank pit and then run through the LAA wall and eventually go to the Control Room, where they read out on temperature instruments. The thermocouples are typically very reliable, but thermocouple failure necessitating thermocouple replacement is very difficult and time-consuming. Instrument failure could potentially cause significant delays as well. Thermocouple or temperature instrument failure would prevent use of T-72 as an evaporator.

b. T-72 steam jacket and associated steam and condensate lines and valvesUsed for heating the tank for evaporation. If the steam supply to the evaporator is shut down for any reason, T-72 cannot be evaporated. Manual valves that allow recirculating cooling water (RCW) or steam to flow through the heating/cooling jacket on T-72 are located in the MUA. There is also a solenoid valve on the condensate discharge from T-72 that is operated by an electrical switch in the Control Room. Typically, replacement parts are available for these valves, and most repairs are accomplished in less than $4 \mathrm{~h}$.

c. PIC-T72-The steam supply to T-72 is controlled at the main panel board in the Control Room by pressure indicator controller (PIC-T72), which supplies air pressure to a steam regulator, pressure control valve (PCV-T72), on the steam supply line to the T-72 steam jacket. Typically, the PICs are very 
reliable and rarely need repair or replacement. The PCVs can sometimes have diaphragm failures and leaks that require repair and occasional replacement. This can take up to $24 \mathrm{~h}$ depending on the extent of the repair.

d. T-72 RCW lines and valves-Used for cooling the tank. The RCW system supplies cooling water to T-72. Manual valves that allow RCW or steam to flow through the heating/cooling jacket on T-72 are located in the MUA. Typically, replacement parts are available for theses valves, and most repairs are accomplished in less than $4 \mathrm{~h}$.

e. Condensers on the VOG line-The two condensers servicing T-72 serve different functions, H-72B has steam supplied to it to prevent the vapor produced during evaporation from condensing and falling back into T-72, and H-72A has RCW supplied to it for condensing the vapor in the condensate line. The steam supply to H-72B is controlled by manual valves and an electrically operated solenoid valve located in the MUA. The RCW supply to H-72A is controlled by manual valves located in the MUA. The operability of the two condensers is very reliable. Spare parts are readily available for these valves, and most repairs are accomplished in less than $4 \mathrm{~h}$.

f. VOG-Used for ventilating the tank. During evaporation, vapor travels through condensers, and the vapor is condensed and collected in a condensation collection tank. This line is intact, and no known reliability issues exist.

\subsection{CLEANEX Operations}

\subsection{Chemical Make Ups}

2.1.1 M-1, M-2, or both are typically used for the make up of the CLEANEX acid strip solutions (hydrochloric acid $[\mathrm{HCl}]$ or nitric acid $\left[\mathrm{HNO}_{3}\right]$ ). These are the same two tanks utilized for the $\mathrm{NaNO}_{3}$ make up used for the target rod dissolution. Refer to Sections 1.2.1 and 1.2.2, respectively, for a discussion of these two tanks and their associated lines and valves.

2.1.2 M-3 is typically used for the make up of the CLEANEX extractant solution, which consists of $1 M$ HDEHP [di-(2-ethylhexyl) phosphoric acid in Exxsol ${ }^{\mathrm{TM}}$ D60. M-3 is a nominal $70 \mathrm{~L}$ Penton tank equipped with an electric stirrer for mixing the tank contents and a "bull's eye" located at the bottom drain line for separation of aqueous and organic solutions. The stirrer consists of an electric Graham motor positioned at the top of $\mathrm{M}-3$, a $90^{\circ}$ gear box, and stirrer. The Graham motor has been in service for over 40 years and has been routinely maintained and has required intermittent repairs in the past. If the Graham electric motor were to fail, a suitable equivalent would need to be specified because it is doubtful that an exact replacement could be procured. The valves associated with M-3 are typically Hayward PVC ball valves. The lines and valves associated with M-3 are listed below:

a. V-M3-1, vent line to VOG 
b. V-M3-2, solution addition line through the funnel

c. V-M3-4, outlet line/drain from bottom of bull's eye

d. V-M3-13, outlet line/drain from between bottom of the tank and top of the bull's eye

2.1.3 M-703 is a vacuum/pressure transfer tank used for transferring organic solutions from drums containing organic chemicals located in an adjacent room to the MUA. M-703 is a nominal $14.5 \mathrm{~L}$ volume glass column (4 in. diameter $\times 72$ in. tall). Typically, the glass column tanks require very little maintenance. Replacement of the flange gaskets on glass column tanks is required very infrequently. The valves associated with M-703 are typically Hayward PVC ball valves. The ball valves sometimes develop leaks, but typically they are very reliable. Normally, leaking valves can be repaired, but replacement is usually about as cost-effective as repairing. The lines and valves associated with the tank used for the CLEANEX extractant make up are listed below:

a. V-M703-1, vent line to VOG

b. V-M703-2, vacuum supply

c. V-M703-3, 5 psig air pressure supply line

d. V-M703-4, outlet line/drain from the bottom of the tank

e. V-M703-9, HDEHP inlet

f. V-M703-10, HDEHP inlet at drum inside flammable storage cabinet in drum room

g. V-C705-1, Exxsol ${ }^{\mathrm{TM}}$ D60 treatment column vent line to VOG

h. V-C705-2, inlet from Exxsol ${ }^{\text {TM }}$ D60 drum in flammable storage cabinet in the drum room

i. V-C705-4, outlet from Exxsol ${ }^{\mathrm{TM}}$ D60 treatment column

2.1.4 M-70 — A pressure transfer tank used for transferring aqueous chemicals to tanks in the tank pit and cubicles. M-70 was originally a Penton coated tank that had been in service for greater than 45 years. It was recently replaced with a 6 in. diameter $\times 48$ in. glass column. M-70 is a nominal $20 \mathrm{~L}$ volume tank. M-70 is used for transferring many acidic solutions. The valves associated with M-70 are typically Hayward PVC ball valves. The ball valves sometimes develop leaks, but typically they are very reliable. Normally, leaking valves can be repaired, but replacement is usually about as cost-effective as repairing. The lines and valves associated with M-70 are listed below:
a. V-M70-1, vent line to VOG
b. V-M70-2, solution addition line through the funnel
c. V-M70-3, 5 psig air pressure supply line
d. V-M70-4, outlet line/drain from the bottom of the tank
e. V-M70-5, demineralized water addition line
f. V-M70-13, outlet line 
2.1.5 M-71-A pressure transfer tank used for transferring aqueous and organic chemicals to tanks in the tank pit and cubicles. M-71 is a Penton lined and coated tank that has been in service for greater than 45 years. M-71 is a nominal $52 \mathrm{~L}$ volume tank. The valves associated with M-71 are typically Hayward PVC ball valves. The ball valves sometimes develop leaks, but typically they are very reliable. Normally, leaking valves can be repaired, but replacement is usually about as cost-effective as repairing. The lines and valves associated with M-71 are listed below:

a. V-M71-1, vent line to VOG

b. V-M71-2, solution addition line through the funnel

c. V-M71-3, 5 psig air pressure supply line

d. V-M71-4, outlet line

e. V-M71-6, inlet line

f. V-M71-13, outlet line

2.1.6 M-75-A pressure transfer tank used for transferring organic chemicals to tanks in the tank pit and cubicles. M-75 is a Penton lined and coated tank that has been in service for greater than 45 years. M-75 is a nominal $71 \mathrm{~L}$ volume tank. The valves associated with M-75 are typically Hayward PVC ball valves. The ball valves sometimes develop leaks, but typically they are very reliable. Normally, leaking valves can be repaired, but replacement is usually about as cost-effective as repairing. The lines and valves associated with M-75 are listed below:

a. V-M75-1, vent line to VOG

b. V-M75-2, solution addition line through the funnel

c. V-M75-3, 5 psig air pressure supply line

d. V-M75-4, outlet line/drain from the bottom of sight glass

e. V-M75-7, outlet line

f. V-M75-10, sight glass isolation valve

g. V-M75-12, inlet valve

h. V-M75-13, outlet line

\subsection{CLEANEX Feed Adjustment}

2.2.1 T-72 is typically the feed tank for the CLEANEX operations. Refer to Sections 1.6.2 and 1.6.3 for a complete discussion of Tank T-72.

2.2.2 M-764 is a MUA tank that is used to meter water into T-72 at a controlled rate. M-764 is a 4 in. diameter $\times 48$ in. tall glass column. M-764 is a nominal $9.7 \mathrm{~L}$ tank. Demineralized water is added to T-72 through lines, valves, and FCVM764 at a controlled rate during evaporation to drive off excess acid. The flow control system consists of a flow controller/recorder, a flow transmitter, and FCV. This solution addition system has proven to be reliable with spare parts readily available, and repairs can typically be made without incurring significant down time. The manual valves associated with M-764 are typically Jamesbury 
ball valves. The ball valves sometimes develop leaks, but they are typically very reliable. The lines and valves associated with M-764 are listed below:
a. V-M764-1, vent line to VOG
b. V-M764-2, solution addition line through the funnel
c. V-M764-3, 5 psig air pressure supply line
d. V-M764-4, drain line/solution outlet
e. V-M764-6, spare inlet
f. V-M764-7, outlet to FCV-M764
g. V-M764-10, FCV bypass
h. V-M764-11, outlet line
i. V-M764-12, discharge from FCV-M764 to LR-7
j. V-M764-13, spare outlet line

2.2.3 M-704 and M-706 are MUA tanks (used for the addition of $\mathrm{HCl}$ and sodium hypochlorite [ $\mathrm{NaOCl}]$ ). Refer to Section 1.4.2 for discussion of M-704. M-706 is a flanged 2 in. diameter $\times 48$ in. long glass column with flanges on each end. M706 is a nominal 2.4 L tank. Inlet lines into M-706 come in through the top flange and there is one outlet line exiting through the bottom flange. The inlet lines and outlet line have Haywood PVC ball valves for air pressure, water and venting utilities, solution addition, and solution discharge. Typically, this type tank requires minimal maintenance, and spare parts are readily available if required. The ball valves sometimes develop leaks, but typically they are very reliable. Normally, leaking valves can be repaired, but replacement is usually about as cost-effective as repairing. The lines and valves associate with these tanks are listed below:
a. V-M706-1, vent line to VOG
b. V-M706-2, solution addition line through the funnel
c. V-M706-3, 5 psig air pressure supply line
d. V-M706-4, drain line/solution outlet
e. V-M706-7, demineralized water addition line

\subsection{CLEANEX Extraction, Scrubbing, Stripping, and Organic Cleanout Operations}

\subsubsection{CLEANEX Extraction}

a. CLEANEX extractant ( $1 M$ HDEHP in Exxsol ${ }^{\text {TM }}$ D60) is added to T-72 once the feed adjustment is confirmed complete. The acid concentration of the solution is adjusted to a low acid concentration using additions of $\mathrm{NaOH}$ solution. At the low acid, the actinide product extracts into the organic from the aqueous solution. The $\mathrm{NaOH}$ additions are typically made from $\mathrm{M}-704$ or M-706. These two tanks are discussed in Sections 1.4.2 and 2.2.3.

b. Samples are pulled from T-72 during the extraction to confirm that the extraction is complete. T-72 sampling is discussed in Section 1.6.2.e. 


\subsubsection{CLEANEX Extraction Raffinate Transfer}

Once the extraction is confirmed complete, the extraction raffinate is transferred (phase separated) from T-72 to a collection tank (usually T-21 or T-23). The transfer route is from T-72 to T-23 using T-734 vacuum/pressure tank on RR-7 to perform the transfer.

Cell equipment utilized:

a. Special FDV at FDV-T70-2 position (line at top) on BR-7-With polyethylene line connected; fabricated in house. Typically spares are available.

b. T73402 GN on RR-7-Standard Ta GN with polyethylene line connected to special FDV at FDV-T70-2. Previously discussed in Section 1.6.1.d.

c. PS-733 position on RR-7-Phase separator with slide valve installed in position. Failure of a phase separator (PS) is rare. Failure of the O-rings on a slide valve is the typical failure mode. Replacement of the slide valve Orings is accomplished rather quickly inside the hot cell cubicle. Spare replacement O-rings are kept in stock. The PSs and slide valves are fabricated in house and are limited to an adequate number of spare PSs that are available for replacement if a PS exhibits a catastrophic failure.

d. Special 2-line block at HV-T734-2 position on RR-7-Inlet valve for T-734 tank (Previously discussed in Section 1.6.1.f.) with polyethylene line and PVC ball valve connecting both lines.

e. T-734 located on RR-7-Previously discussed in Section 1.6.1.g.

f. Special 2-line block at HV-T734-3 position on RR-7-Outlet valve for T734 tank (Previously discussed in Section 1.6.1.h.) with polyethylene line and PVC ball valve connecting both lines.

g. T73404 GN on RR-7-Standard Ta GN (Previously discussed in Section 1.6.1.i) with polyethylene line connected to C4C601 GN (if going to T-23) or C7403 GN (if going to T-21); fabricated in house. Typically spares are available.

h. C4C601 GN in Cubicle 6 left DC well-Standard Zr GN to hard piped jumper between Cubicle 6 DC well and Cubicle 4 DC well with polyethylene line connected from T73404 GN on RR-7. Typically, spares are readily available.

i. C4C601 jumper-Hard piped jumper between Cubicle 6 left DC well and Cubicle 4 left DC well. If the jumper develops a leak, it most likely cannot be repaired or replaced and an alternative route must be used such as a polyethylene line run through the cubicle pass through openings.

j. C4C601 jumper-Hard piped jumper located in Cubicle 4, from Cubicle 4 left DC well to C4C601 GN on BR-4. If the jumper develops a leak, it most likely cannot be repaired or replaced, and an alternative route must be used, such as removing the jumper end from the DC well position and installing a GN in the DC well position. 
k. C4C601 GN on BR-4-Standard Zr GN with polyethylene line connected to T2302 GN in Cubicle 4 right DC well. Typically, spares are readily available.

TO T-23:

l. T2302 GN in Cubicle 4 right DC well—Standard Zr GN with polyethylene tubing connected to C4C601 GN on BR-4. Fabricated in house. Typically spares are available.

TO T-21:

m. C7403 GN in Cubicle 7 left DC well-Standard Zr GN (C7403 line is teed below the cubicle floor to T2103 line to T2103 GN in Cubicle 6 right DC well); fabricated in house. Typically spares are available. If a leak develops in the tee line, then use of T2103GN and C7403 GN would have to be abandoned.

n. T2103 GN in Cubicle 6 right DC well—Standard Zr GN (T2103 line is teed below the cubicle floor to C7403 line to C7403 GN in Cubicle 7 left DC well); fabricated in house. Typically spares are available. If a leak develops in the tee line, then use of T2103GN and C7403 GN would have to be abandoned.

o. HV-T734-1on RR-7-Refer to Section 1.6.1.k for discussion of HV-T734-1.

p. KP-735 on RR-7-A KOP for the T-734 vacuum/pressure transfer system. Previously discussed in Section 1.6.1.I.

q. CU7AJ_-In-cell air jet used for vacuum source (Previously discussed in Section 1.4.1.p.).

r. Polyethylene tubing - For vacuum lines, pressure lines, demineralized water lines, and portions of the transfer route. Male ball joints, and plastic compression fittings are readily available. Previously discussed in Section

1.4.1.q above.

\subsubsection{CLEANEX Extraction Raffinate and Scrub Receiving Tank, T-21}

T-21 has several systems that are required to operate properly to maintain functionality. Typically, tank pit tanks like T-21 do not have many physical or mechanical issues that interrupt routine operations. Plugging instrument probes or broken instrument lines can pose some minor problems, but these problems are a low probability.

a. T-21-A nominal $155 \mathrm{~L}$ tank constructed of HC. T-21 is located in Tank Pit 2 and is used to collect CLEANEX extraction raffinate and scrubs and solutions for CLEANEX extractions and recovery.

b. T-21 instrument lines and process lines - Lines in the tank are constructed of $\mathrm{HC}$ with the exception of the inlet lines that are constructed of $\mathrm{Zr}$.

c. Foxboro pneumatic instrument-T-21's liquid level and density are read out and recorded on an original Foxboro pneumatic instrument (over 40 years 
old). This instrument is typically reliable and is routinely calibrated. Broken polyethylene lines supplying the pneumatic signals to the transmitters and instrument can present some problems, but in recent years, UV-resistant polyethylene has decreased potential breaking of lines due to age and exposure to UV light. Plugged lines can be a problem if the plug cannot be removed easily.

d. T-21 sparger-An electric switch activates a solenoid that opens and allows air to flow into a deep leg in the tank for solution mixing in the tank. The air flow into the tank is regulated by an orifice plate in the sparge line. The orifice plate can plug requiring the suspension of sparging operations in $\mathrm{T}$ 21. The orifice plate would have to be removed and cleaned. This typically only requires no more than $4 \mathrm{~h}$. Plugs in the sparge line downstream of the orifice or flow meter can occasionally occur. This can sometimes require as many as 12-24 h to clear. The sparger solenoid has been known to fail, even though failure is not frequent. Rebuilds and replacements are readily available and cause very minimal down time for repairs. If necessary an alternate method of sparging T-21 is by using the in-cell air and applying air pressure down the $\mathrm{T}-21$ sample uptake line.

e. T-21 processing lines-These are the lines that are used to transfer process solutions into and out of T-21. Typically, these lines are not an issue, though at times some lines are preferred over others, and plugged lines can be a problem if the plug cannot be removed easily or an alternate line cannot be used.

f. T-21 sampler-A $1 \mathrm{~L}$ glass bottle with a 2-line rubber stopper for sampling in Cubicle 4 is connected to a polyethylene line that is connected to a standard Zr GN installed in the T2102 position in the Cubicle 6 right disconnect well. The T2102 jumper has been removed because the T2102 line on the sample rack is leaking. Glass bottles, stoppers, polyethylene tubing with female ball joints, polyethylene tubing, and plastic compression fittings are kept in stock and typically readily available. (The designed sample technique is no longer employed because the T-21 vacuum accumulator and needle block position [SA-T21] are out of service.) The T21 sampler is out of service.

\subsubsection{CLEANEX Extraction Raffinate and Scrub Receiving Tank, T-23}

T-23 has several systems that are required to operate properly to maintain functionality. Typically, tank pit tanks like T-23 do not have many physical or mechanical issues that interrupt routine operations. Plugging instrument probes or broken instrument lines can pose some minor problems, but these problems are a low probability.

a. T-23-A nominal $152 \mathrm{~L}$ tank constructed of HC. T-23 is located in Tank Pit 2 and is used to collect CLEANEX extraction raffinate, scrubs, and solutions for CLEANEX extractions and recovery. 
b. T-23 instrument lines and process lines-Lines in the tank are constructed of $\mathrm{HC}$ with the exception of the inlet lines that are constructed of $\mathrm{Zr}$.

c. Foxboro pneumatic instrument-T-23's liquid level and density are read out and recorded on an original Foxboro pneumatic instrument (over 40 years old). This instrument is typically reliable and is routinely calibrated. Broken polyethylene lines supplying the pneumatic signals to the transmitters and instrument can present some problems, but in recent years, UV-resistant polyethylene has decreased potential breaking of lines due to age and exposure to UV light. Plugged lines can be a problem if the plug cannot be removed easily.

d. T-23 sparger-An electric switch activates a solenoid that opens and allows air to flow into a deep leg in the tank for solution mixing in the tank. The air flow into the tank is regulated by an orifice plate in the sparge line. The orifice plate can plug, requiring the suspension of sparging operations in T23. The orifice plate would have to be removed and cleaned. This typically only requires no more than $4 \mathrm{~h}$. Plugs in the sparge line downstream of the orifice or flow meter can occasionally occur. This can sometimes require as many as $12-24$ h to clear. The sparger solenoid has failed, even though failure is not frequent. Rebuilds and replacements are readily available and cause very minimal down time for repairs. If necessary, an alternate method of sparging T-23 is by using the in-cell air and applying air pressure down the T-23 sample uptake line.

e. T-23 processing lines-These are the lines that are used to transfer process solutions into and out of T-23. Typically, these lines are not an issue, though at times some lines are preferred over others, and plugged lines can be a problem if the plug cannot be removed easily or an alternate line cannot be used.

f. T-23 sampler-A $1 \mathrm{~L}$ glass bottle with a 2-line rubber stopper for sampling in Cubicle 4 is connected to a polyethylene line that is connected to a standard Zr GN installed in the T2302 position in the Cubicle 4 right disconnect well. The T2302 jumper has been removed due to the T2302 line on the sample rack leaking. Glass bottles, stoppers, polyethylene tubing with female ball joints, polyethylene tubing, and plastic compression fittings are kept in stock and typically readily available. (The designed sample technique is no longer employed since T-23 vacuum accumulator and needle block position [SA-T23] are out of service.)

\subsubsection{CLEANEX Scrubbing}

a. Two $0.03 M$ acid scrubs are added to T-72 from M-70 via the T-72 decontamination line (V-T72-4).

b. Each scrub is sparged, settled, and then sampled in T-72 to confirm that the scrubs meets acidity specifications and to determine activity levels in the aqueous scrub solution. T-72 sampling is discussed in Section 1.6.2.e. 
c. Once the scrub is confirmed to meet specifications, each scrub is transferred (phase separated) from T-72 to a collection tank (usually T-21 or T-23). The transfer route is from T-72 to T-23 using T-734 vacuum/pressure tank on RR-7 to perform the transfer. The transfer route is discussed in Section 2.3.2.

d. Once scrubbing is complete, phase modifier ( $0.2 M$ basic Alamine 336 in Exxon Aromatic 150) is made up utilizing the M-703 vacuum/pressure transfer system. Refer to Section 2.1.3 for discussion of this system. The $0.2 M$ Alamine 336 in Exxon Aromatic 150 is made up in the fume hood and contacted with $6 \mathrm{M} \mathrm{HCl}$ to acidify the phase modifier. The phase modifier is then added to T-72 from M-71 via V-M71-9 to retain the Fe constituent during stripping operations. Refer to Section 2.1.5 for discussion of M-71.

\subsubsection{CLEANEX Stripping}

NOTE: Eight strips of the T-72 pregnant organic are programed. Additional strips may be added to meet desired activity goals for stripping.

a. Each strip solution is added using the same equipment and route as the scrubs in Section 2.3.5.

b. Each strip is sparged and settled in T-72, and the eighth strip solution is sampled for activities. T-72 sampling is discussed in Section 1.6.2.e.

c. Once the strip sample results meet the desired activity limits, the organic phase in T-72 is sampled. T-72 sampling is discussed in Section 1.6.2.e.

\subsubsection{CLEANEX Strip Transfer (T-72 to T-604)}

Each strip is transferred (phase separated) from T-72 to T-604 and then from T604 to a strip collection tank (usually a small evaporator like T-43) to prevent the transfer of any entrained "pregnant organic" to the strip collection tank. The transfer route from T-72 to T-604 utilizes T-734 vacuum/pressure tank on RR-7 to perform the transfer.

Cell equipment utilized:

a. Special FDV at FDV-T70-2 position on BR-7-Previously discussed in Section 2.3.2.a.

b. T73402 GN on RR-7-Standard Ta GN (Previously discussed in Section

1.6.1.d.) with polyethylene line connected to special FDV at FDV-T70-2 position.

c. PS-733 position on RR-7-Previously discussed in Section 2.3.2.c.

d. HV-T734-2 position on RR-7-Previously discussed in Section 1.6.1.f.

e. T-734 on RR-7-Previously discussed in Section 1.6.1.g.

f. HV-T734-3 position on RR-7-Previously discussed in Section 1.6.1.h.

g. T73404 GN on RR-7-Standard Ta GN (Previously discussed in Section

1.6.1.i.) with polyethylene line connected to C7405 GN.

h. C7405 GN in Cubicle 7 left DC well—Standard Ta GN to go to T-604.

i. HV-T734-1-Previously discussed in Section 1.6.1.k. 
j. KP-735-Previously discussed in Section 1.6.1.l.

k. CU7AJ-In-cell air jet used for vacuum source. Previously discussed in Section 1.4.1.p.

1. Polyethylene tubing-For vacuum lines, pressure lines, demineralized water lines, and portions of the transfer route. Male ball joints and plastic compression fittings are readily available. Previously discussed in Section

\subsection{1.q}

\subsubsection{CLEANEX Strip Intermediate Receiving Tank, Evaporator T-604}

T-604 has several systems that are required to operate properly to maintain functionality. Typically, tank pit tanks like T-604 do not have many physical or mechanical issues that interrupt routine operations. Loss of steam and cooling water capabilities could have a negative impact, but this is a low probability.

a. T-604-A nominal $22 \mathrm{~L}$ HC shell with Ta liner evaporator tank located in Tank Pit 6. T-604 has an annulus filled with Hg for increased heat transfer capability. Used as an intermediate transfer tank to remove entrained organic from the aqueous strip product solution.

b. Foxboro pneumatic instrument-T-604's liquid level and density are read out and recorded on an original Foxboro pneumatic instrument (over 40 years old). This instrument is typically reliable and is routinely calibrated. T-604 pressure is read out on an original Foxboro pneumatic instrument (also over 40 years old) that provides read outs for several tank pressures. Specific tank pressures provided on this instrument are selected for read out by a selector switch. Broken polyethylene lines supplying the pneumatic signals to the transmitters and instrument can present some problems, but in recent years, UV-resistant polyethylene has decreased potential breaking of lines due to age and exposure to UV light. Plugged lines can be a problem if the plug cannot be removed easily.

c. T-604 sparger-An electric switch activates a solenoid that opens and allows air to flow into a deep leg in the tank for solution mixing in the tank. The air flow into the tank is regulated by an orifice plate in the sparge line. The orifice plate can plug requiring the suspension of sparging operations in T604 . The orifice plate would have to be removed and cleaned. This typically only requires no more than $4 \mathrm{~h}$. Plugs in the sparge line downstream of the orifice or flow meter can occasionally occur. This can sometimes require as many as $12-24 \mathrm{~h}$ to clear. The sparger solenoid has failed, even though failure is not frequent. Rebuilds and replacements are readily available and cause very minimal down time for repairs. If necessary an alternate method of sparging T-604 is by using the in-cell air and applying air pressure down the T-604 sample uptake line.

d. T-604 processing lines-These are the Ta lines that are used to transfer process solutions into and out of T-604. Typically, these lines are not an issue, though at times some lines are preferred over others, and plugged lines 
can be a problem if the plug cannot be removed easily or an alternate line cannot be used.

e. T-604 sampler-A $1 \mathrm{~L}$ glass bottle with a 2-line rubber stopper for sampling in Cubicle 6 is connected to a 1-line polyethylene block installed in the T604 needle block position (SA-T604) on BR-6 using polyethylene tubing. Glass bottles, stoppers, polyethylene tubing with female ball joints, polyethylene tubing, and plastic compression fittings are kept in stock and typically readily available. (The designed sample technique is no longer employed because there are no needle blocks available to use the vacuum accumulators to pull samples from the tank pit tanks.)

\subsubsection{T-604 Heating/Cooling}

a. Thermocouples-For monitoring the temperature during evaporation and feed adjustment. The thermocouples come off the tank in the tank pit and then run through the LAA wall and eventually go to the Control Room, where they read out on temperature instruments. The thermocouples are typically very reliable, but thermocouple failure necessitating thermocouple replacement is very difficult and time-consuming. Instrument failure could potentially cause significant delays as well. Thermocouple or temperature instrument failure would prevent use of T-604 as an evaporator.

b. T-604 steam jacket and associated steam and condensate lines and valvesUsed for heating the tank for evaporation. If the steam supply to the evaporator is shut down for any reason, T-604 cannot be evaporated. Manual valves that allow RCW or steam to flow through the heating/cooling jacket on T-604 are located in the MUA. There is also a solenoid valve on the condensate discharge from T-604 that is operated by an electrical switch in the Control Room. Typically, replacement parts are available for these valves, and most repairs are accomplished in less than $4 \mathrm{~h}$.

c. PIC-T604-The steam supply to T-604 is controlled at the main panel board in the Control Room by a pressure indicator controller (PIC-T604), which supplies air pressure to a steam regulator, pressure control valve (PCVT604), on the steam supply line to the T-604 steam jacket. Typically, the PICs are very reliable and rarely need repair or replacement. The PCVs can sometimes have diaphragm failures and leaks that require repair and occasional replacement. This can take up to $24 \mathrm{~h}$ depending on the extent of the repair.

d. T-604 RCW lines and valves-Used for cooling the tank. The RCW system supplies cooling water to T-604. Manual valves that allow RCW or steam to flow through the heating/cooling jacket on T-604 are located in the MUA. Typically, replacement parts are available for theses valves, and most repairs are accomplished in less than $4 \mathrm{~h}$.

e. Condenser on the VOG line-The H-604 condenser is located in the tank pit and has RCW supplied to it for condensing the vapor in the condensate line. The RCW supply to H-604 is controlled by manual valves located in the 
MUA. The operability of the condenser is very reliable. Spare parts are readily available for these valves, and most repairs are accomplished in less than $4 \mathrm{~h}$.

f. VOG-Used for ventilating the tank. During evaporation, vapor travels through condensers and the vapor is condensed and collected in a condensation collection tank. This line is intact, and no known reliability issues exist.

\subsubsection{CLEANEX Strip Transfer (T-604 to T-43)}

Once the strip solution is received into T-604, each strip is sparged and settled in T-604. Each strip is transferred (phase separated) from T-604 to a strip collection tank (usually a small evaporator like T-43). The transfer route from T-604 to T-43 utilizes P-653 vacuum/pressure diaphragm pump on BR-6 to perform the transfer.

Cell equipment utilized:

a. 7PDV-604-1 (Type D set to 7) on BR-6-Fabricated in house. Typically spares are available.

b. PS-604 on BR-6-A PS with slide valve installed in position. Failure of a PS is rare. Failure of the O-rings on a slide valve is the typical failure mode. Replacement of the slide valve O-rings is accomplished rather quickly inside the hot cell cubicle. Spare replacement O-rings are kept in stock. The PSs and slide valves are fabricated in house and are limited to an adequate number of spare PSs that are available for replacement if a PS exhibits a catastrophic failure.

c. 2-line block at HCV-P604-1 position on BR-6 with PVC valve and polyethylene line connected to the right side-Fabricated in house. Typically spares are available.

d. P65301 GN on BR-6 - Standard Zr GN with polyethylene line connected from 2-line block at HCV-P604-1 position on BR-6. Fabricated in house. Typically spares are available.

e. P-653 vacuum/pressure diaphragm pump on BR-6 (referred to as a TRU pump because it was designed specifically for use in this facility)—No real spares available. Pumps can be rebuilt with significant effort. There are several nonfunctioning spare pumps available for rebuilding. Current Hot Cell Operations personnel have little or no experience in rebuilding this type of pump. Spare parts to rebuild the pumps are limited at this writing.

f. P65302 GN on BR-6-Standard Zr GN with PVC valve and polyethylene line connected to special FDV at FDV-T604 on BR-6; fabricated in house. Typically spares are available.

g. FDV-T604 position with special FDV (line at left) on BR-6-Fabricated in house. Typically spares are available. 
h. 7PDV-604-2 (Type D set to 2) on BR-6-Seven position diversion valve set for pump transfer route flush. Fabricated in house. Typically spares are available.

i. HCV-P604-2 position (utilizing a PVC block toggle hand valve) on BR-6Fabricated in house. Typically spares are available. (2-line block with PVC ball valve and polyethylene line connecting both sides may be installed if desired.)

j. $\quad$ Polyethylene tubing-For vacuum lines, pressure lines, demineralized water lines, and portions of the transfer route. Male ball joints and plastic compression fittings are readily available. Previously discussed in Section

\subsection{1.q.}

\subsubsection{CLEANEX Strip Product Receiving Tank, Evaporator T-43}

T-43 has several systems that are required to operate properly to maintain functionality. Typically, tank pit tanks like T-43 do not have many physical or mechanical issues that interrupt routine operations. Loss of steam and cooling water capabilities could have a negative impact, but this is a low probability.

a. T-43-A nominal 22 L HC shell with Ta liner evaporator tank located in Tank Pit 4. T-43 has an annulus filled with Hg for increased heat transfer capability and is used to evaporate the CLEANEX strip product.

b. Foxboro pneumatic instrument-T-43's liquid level and density are read out and recorded on an original Foxboro pneumatic instrument (over 40 years old). This instrument is typically reliable and is routinely calibrated. T-43 pressure is read out on an original Foxboro pneumatic instrument (also over 40 years old) that provides read outs for several tank pressures. Specific tank pressures provided on this instrument are selected for read out by a selector switch. Broken polyethylene lines supplying the pneumatic signals to the transmitters and instrument can present some problems, but in recent years, UV-resistant polyethylene has decreased potential breaking of lines due to age and exposure to UV light. Plugged lines can be a problem if the plug cannot be removed easily.

c. T-43 sparger-An electric switch activates a solenoid that opens and allows air to flow into a deep leg in the tank for solution mixing in the tank. The air flow into the tank is regulated by an orifice plate in the sparge line. The orifice plate can plug requiring the suspension of sparging operations in $\mathrm{T}$ 43. The orifice plate would have to be removed and cleaned. This typically only requires no more than $4 \mathrm{~h}$. Plugs in the sparge line downstream of the orifice or flow meter can occasionally occur. This can sometimes require as many as $12-24$ h to clear. The sparger solenoid has failed, even though failure is not frequent. Rebuilds and replacements are readily available and cause very minimal down time for repairs. If necessary an alternate method of sparging $\mathrm{T}-43$ is by using the in-cell air and applying air pressure down the T-43 sample uptake line. 
d. T-43 processing lines-These are the Ta lines that are used to transfer process solutions into and out of T-43. Typically, these lines are not an issue, though at times some lines are preferred over others, and plugged lines can be a problem if the plug cannot be removed easily or an alternate line cannot be used.

e. T-43 sampler-A $1 \mathrm{~L}$ glass bottle with a 2-line rubber stopper for sampling in Cubicle 4 is connected to a polyethylene line that is connected to a standard Ta GN installed in the T4302 position in the Cubicle 4 right disconnect well. The T4302 jumper has been removed due to the T4302 line on the sample rack leaking. Glass bottles, stoppers, polyethylene tubing with female ball joints, polyethylene tubing, and plastic compression fittings are kept in stock and typically readily available. (The designed sample technique is no longer employed since T-43 vacuum accumulator and needle block position [SA-T43] are out of service.)

\subsubsection{T-43 Heating/Cooling}

a. Thermocouples-For monitoring the temperature during evaporation and feed adjustment. The thermocouples come off the tank in the tank pit and then run through the LAA wall and eventually go to the Control Room, where they read out on temperature instruments. The thermocouples are typically very reliable, but thermocouple failure necessitating thermocouple replacement is very difficult and time-consuming. Instrument failure could potentially cause significant delays as well. Thermocouple or temperature instrument failure would prevent use of T-43 as an evaporator.

b. T-43 Steam jacket and associated steam and condensate lines and valvesUsed for heating the tank for evaporation. If the steam supply to the evaporator is shut down for any reason, T-43 cannot be evaporated. Manual valves that allow RCW or steam to flow through the heating/cooling jacket on T-43 are located in the MUA. There is also a solenoid valve on the condensate discharge from T-43 that is operated by an electrical switch in the Control Room. Typically, replacement parts are available for these valves, and most repairs are accomplished in less than $4 \mathrm{~h}$.

c. PIC-T43-The steam supply to T-43 is controlled at the main panel board in the Control Room by pressure indicator controller (PIC-T43), which supplies air pressure to a steam regulator, pressure control valve (PCV-T43), on the steam supply line to the T-43 steam jacket. Typically, the PICs are very reliable and rarely need repair or replacement. The PCVs can sometimes have diaphragm failures and leaks that require repair and occasional replacement. This can take up to $24 \mathrm{~h}$ depending on the extent of the repair.

d. T-43 RCW lines and valves-Used for cooling the tank. The RCW system supplies cooling water to T-43. Manual valves that allow RCW or steam to flow through the heating/cooling jacket on T-43 are located in the MUA. Typically, replacement parts are available for theses valves, and most repairs are accomplished in less than $4 \mathrm{~h}$. 
e. Condenser on the VOG line-The H-43 condenser is located in the tank pit and has RCW supplied to it for condensing the vapor in the condensate line. The RCW supply to $\mathrm{H}-43$ is controlled by manual valves located in the MUA. The operability of the condenser is very reliable. Spare parts are readily available for these valves, and most repairs are accomplished in less than $4 \mathrm{~h}$.

f. VOG-Used for ventilating the tank. During evaporation, vapor travels through condensers and the vapor is condensed and collected in a condensation collection tank. This line is intact, and no known reliability issues exist.

\subsubsection{CLEANEX Confirming Strip}

a. Once the stripping of T-72 is confirmed complete from the T-72 strip sample analysis, a confirming strip (flush) is added to T-604 from M-70 or equivalent tank (M-704 in this case) via Cubicle 7 solution addition line to C7405 GN.

b. The confirming flush is sparged, settled, and sampled in T-604.

c. The confirming flush is transferred from T-604 to T-43 as described in Section 2.3.10.

d. Once the confirming flush sample analysis is determined to meet the activity specification to terminate stripping, the organic phase in T-604 is sampled. T-604 sampling is discussed in Section 2.3.8.e.

e. T-43 strip product evaporations are routinely performed during the stripping operations. Refer to Sections 2.3.11 and 2.3.12 for a discussion of the Tank T-43 evaporator.

\subsubsection{CLEANEX Accountability Sampling in Tank T-43}

a. Once the last strip and T-604 confirming flush are collected in T-43, stripping has been confirmed complete, and T-43 is at a high volume (>12 L), T-43 is sparged and accountability (duplicate) samples are pulled from T-43. Previously discussed in Section 2.3.11.e.

b. The liquid level and density of T-43 is measured using a calibrated digital manometer (Merical) to determine an accurate volume that is used for determining accurate isotopic quantities in T-43. Broken or plugged instrument lines (polyethylene sections located in the MUA are the typical issues) can cause operational delays, but these are typically of short duration. The broken polyethylene lines are usually found quickly and repaired/replaced. The plugged instrument lines are usually cleared quickly as well. Previously discussed in Section 2.3.11.b.

\subsubsection{CLEANEX Organic Cleanout}

Once stripping operations have been confirmed complete based on T-72 and T604 sample results, organic cleanouts of these tanks may be performed. 
a. M-704 and M-706 are typically used to make an addition of a specified volume of low concentration $(<5 M) \mathrm{NaOH}$ to T-72 and T-604 to neutralize the acidic aqueous heel and acidic organic for transfer to F-126 waste tank. M-704 and M-706 are discussed in Sections 1.4.2 and 2.2.3, respectively.

b. The tanks are sparged and sampled to confirm that the solutions are neutralized or slightly basic. The T-72 sampler is discussed in Section 1.6.2.e, and the $T-604$ sampler is discussed in Section 2.3.8.e.

c. T-72 and T-604 contents are transferred to F-126 utilizing T-734 vacuum/pressure tank on RR-7 to perform the transfer. The transfer routes are discussed below in Sections 2.3.16 and 2.3.17.

d. Both T-72 and T-604 are flushed to F-126 with diluent and water using the T-734 vacuum/pressure tank on RR-7 to perform the transfer. The diluent is obtained for the flushing by utilizing the M-703 vacuum/pressure transfer system previously discussed in Section 2.3.1.

e. At times when the waste jets from T-72 and T-604 to the F-115 waste tank are operating, water flushes are added to T-72 and T-604, sparged, and jetted to F-115. Otherwise the flushes are transferred to F-126 via the routes outlined in Section 2.3.16 and 2.3.17. If there is a steam outage, then the steam jets cannot be utilized for transfers. Steam jets can fail and because they are located in the Hot Cell Tank Pits, it is very difficult to replace them, even with the availability of spare jets. At times, the jets can get plugged with debris, and it can require significant effort to unplug it causing operational delays. This is why the vacuum/pressure tank routes to F-126 waste tank from the process tanks are often utilized.

f. Any remaining diluent in T-72 and T-604 is steam distilled by adding water and evaporating off a specified amount of water to T-34, the evaporator condensate collection tank. ( $\mathrm{T}-72$ evaporator operation is discussed in Section 1.6.3, and T-604 evaporator operation is discussed in Section 2.3.9.)

g. The remainder of the water in the tanks after the steam distillation is complete is jetted to F-115 or transferred to F-126 per previously discussed routes. (The transfer routes are discussed in Section 2.3.15.e above.)

\subsubsection{T-72 CLEANEX Waste Organic Transfer}

Once T-72 has been confirmed to be basic (or neutral) based on T-72 sample results, transfer of the waste organic may be performed.

NOTE: Typically, the portion of the transfer route from T-72 to T-734 will not be changed, but it is discussed again below in Sections 2.3.16.a-h. Only the discharge line from $T-734$ will be required to be rerouted to the appropriate collection tanks.

Cell equipment utilized:

a. Special FDV at FDV-T70-2 position on BR-7-Previously discussed in Section 2.3.2.a. 
b. T73402 GN on RR-7-Standard Ta GN. with polyethylene line connected to special FDV at FDV-T70-2 position. Previously discussed in Section 1.6.1.d.

c. PS-733 position on RR-7-Previously discussed in Section 2.3.2.c.

d. HV-T734-2 position (inlet valve for T-734 tank) on RR-7-Previously discussed in Section 1.6.1.f.

e. T-734 on RR-7-Previously discussed in Section 1.6.1.g.

f. HV-T734-3 position on RR-7-Previously discussed in Section 1.6.1.h.

g. T73404 GN on RR-7-Standard Ta GN outlet from T-734 with polyethylene line connected to 1-line block at SA-F126. Previously discussed in Section 1.6.1.i.

h. 1-line block at SA-F126 (F12602 line to F-126) position on BR-7Polyethylene line connected to T73404 GN; fabricated in house. Typically spares are available.

i. HV-T734-1on RR-7-Refer to Section 1.6.1.k for discussion of HV-T734-3.

j. $\quad$ KP-735on RR-7-Previously discussed in Section 1.6.1.l.

k. CU7AJ-In-cell air jet used for vacuum source. Previously discussed in Section 1.4.1.p.

l. Polyethylene tubing — For vacuum lines, pressure lines, demineralized water lines, and portions of the transfer route. Male ball joints and plastic compression fittings are readily available. Previously discussed in Section 1.4.1.q.

\subsubsection{T-604 CLEANEX Waste Organic Transfer}

Once T-604 has been confirmed to be basic (or neutral) based on T-604 sample results, transfer of the waste organic may be performed.

NOTE: Typically, the portion of the transfer route from T-604 to T-734 is the only thing required to be set because the T-734 system was previously used to dispose of organic from T-72, but it is discussed again below in Sections 2.3.17.a-i.

Cell equipment utilized:

a. 7PDV-604-1 (Type D set to 7) on BR-6-Previously discussed in Section 2.3.10.a.

b. Special 2-line block at PS-604 position on BR-6 with polyethylene tubing connected to the left side-Fabricated in house. Typically spares are available.

c. T73402 GN on RR-7-Standard Ta GN (Previously discussed in Section 1.6.1.d.) with polyethylene line connected to the left side of special 2-line block as PS-604 position on BR-6.

d. PS-733 position on RR-7-Previously discussed in Section 2.3.2.c.

e. HV-T734-2 on RR-7-Previously discussed in Section 1.6.1.f.

f. T-734 on RR-7-Previously discussed in Section 1.6.1.g.

g. HV-T734-3 on RR-7-Previously discussed in Section 1.6.1.h. 
h. T73404 GN on RR-7-Standard Ta GN with polyethylene line connected to 1-line block at SA-F126. Previously discussed in Section 1.6.1.i.

i. 1-line block at SA-F126 (F12602 line to F-126) —Located on BR-7 with polyethylene line connected to T73404 GN; fabricated in house. Typically spares are available. Previously discussed in Section 2.3.16.h.

j. $\quad$ HV-T734-1 on RR-7-Previously discussed in Section 1.6.1.k.

k. KP-735on RR-7-Previously discussed in Section 1.6.1.I.

l. CU7AJ-In-cell air jet used for vacuum source. Previously discussed in Section 1.4.1.p.

m. Polyethylene tubing — For vacuum lines, pressure lines, demineralized water lines, and portions of the transfer route. Male ball joints, and plastic compression fittings are readily available. Previously discussed in Section

\subsection{1.q.}

\subsection{Anion Exchange Operations}

3.1 Chemical Make Ups

NOTE: Stock lithium chloride ( $\mathrm{LiCl}$ ) is the main chemical used for the chemical make ups of synthetic feed, rare earth elutriant, and Am-Cm elutriant. All the chemical make ups for the anion exchange operations are made up in nominal 25 L polyethylene bottles.

3.1.1 The $\mathrm{LiCl}$ solution is transferred using the M-600 vacuum/pressure transfer system. Tank M-600, KOP M-602, an inline filter, and associated lines and valves comprise the M-600 vacuum/pressure transfer system. The M-600 system is used for transferring stock $\mathrm{LiCl}$ solutions from a drum into the appropriate chemical make up bottle. M-600 is a nominal 14.5 L volume glass column (4 in. diameter $\times 72$ in. tall). KOP M-602 is a 2 in. diameter $\times 18$ in. long glass column that serves as a KOP to protect the vacuum source if accidental overfilling of M-600 occurs. Typically, the glass column tanks require very little maintenance. Replacement of the flange gaskets on glass column tanks is required very infrequently. The valves associated with M-600 are typically Hayward PVC ball valves. The ball valves sometimes develop leaks, but typically they are very reliable. Normally, leaking valves can be repaired, but replacement is usually about as cost-effective as repairing. The lines and valves associated with the tank used for the stock LiCl transfers are listed below:
a. V-M602-1, vent line to VOG
b. V-M602-2, vacuum supply
c. V-M602-3, 5 psig air pressure supply line
d. V-M602-4, outlet line/drain from the bottom of the KOP
e. V-M600-4, outlet line/drain from the bottom of the tank
f. V-M600-7, inlet line from M-602 into M-600 


\subsection{Preparing Anion Exchange Column C-636}

NOTE: The resin column, C-636, used for LiCl anion exchange operations and located on RR-6 had a companion column, C-648, located on BR-6. C-648 was used primarily for rare earth and residual fission product radioisotope removal from the product material prior to it being run through C-636 anion exchange for the actinide separation. C-648 experienced some plugging problems causing significant restriction in flow through the column once resin was loaded. Several attempts were made to resolve the plugging problems to no avail. That $C-648$ column was removed from BR-6 and discarded to waste. Another column was fabricated for $C-648$, and it was thought to have been fabricated to the original specifications for both C-648 and C-636. After a couple of uses, C-648 once again experienced significant flow restrictions. After attempts to "unplug" this newer C-648 were unsuccessful, it was no longer used for rare earth and residual fission product radioisotope removal. This occurred in the late 1990s. Since then C-636 has assumed the task of handling both its duties and those of C-648. There is no spare column for C-636, and a failure would create many, many weeks of down time until a new column was fabricated, installed, and tested.

3.2.1 If there is any resin in C-636, it is removed utilizing the in-cell demineralized water supply in the Cubicle 6 . The flow route is set to supply water from the demineralized water supply in Cubicle 6 to upflow water through C-636 to remove the used resin into polyethylene bottles in Cubicle 6. New resin is loaded into C-636 from M-63. M-63 is a 4 in. inner diameter (ID) $\times 30$ in. long stainless steel tank with a bull's eye located in line on the discharge line at the bottom of the tank to evaluate the progress of the resin addition. M-63 has a $~ 6 \mathrm{~L}$ nominal volume. The valves associated with M-63 are typically Haywood PVC ball valves, but there are a couple of gate valves associated with the flow indicator, FI-M63. The valves sometimes develop leaks, but typically they are very reliable. Normally, leaking valves can be repaired, but replacement is usually about as cost-effective as repairing. Spare valves are readily available and down time is relatively short ( $\sim 4 \mathrm{~h}$ ). The lines and valves associated with the M-63 tank and the cubicle flow route through C-636 are listed below.

MUA equipment utilized:

a. V-M63-1, vent line

b. V-M63-2, solution addition line through the funnel

c. V-M63-3, 20 psig air pressure supply line

d. V-M63-4, outlet line/drain

e. V-M63-5, outlet line from FI-M63 to Tank M-63

f. V-M63-6, inlet line to FI-M63

g. V-M63-7, outlet line to the L11 disconnect position in Cubicle 6

Cell equipment utilized: 
h. Anion exchange column C-636-A nominal $1.4 \mathrm{~L}$ anion exchange column. C-636 is a 2 in. OD diameter $\times 30$ in. tall $\mathrm{Zr}$ column with a $\mathrm{Zr}$ heating/cooling jacket and a metal frit at the bottom that allows the column to hold resin for performing the anion exchange operations. There are no spares for C-636. This column has been in operation for over 35 years. An identically constructed ion exchange column, C-648, located on BR-6, experienced restricted flow issues between the late 1980s and early 1990s. A new column was fabricated to replace the C-648 column. After only a few ion exchange operations, this new C-648 column started experiencing restricted flow like the old C-648 that it replaced. Extensive effort was expended to resolve the restricted flow issues, but it was never resolved. Currently, the ion exchange column in the C-648 position on BR-6 is not used. This raises concern over being able to produce a new viable replacement column for $\mathrm{C}-636$. If it failed, it could require as long as $2-$ 3 months to procure the necessary materials and have a new ion exchange column fabricated to replace C-636.

i. L11 position on east wall in Cubicle 6-Zr GN. Typically GNs that are permanently installed in cubicle service line TRU disconnects function almost indefinitely without leaking, but spare GNs can be obtained in a very short time if there is a failure of this GN.

j. Special FDV at FDV-636 position (line at left) on RR-6-Fabricated in house. Typically spares are available.

k. 2-line polyethylene block at HCV-C636-1 position-Fabricated in house. Typically spares are available.

l. For resin removal from C-636 connect the Cubicle 6 demineralized water line to special FDV @FDV-C636 with line at the left position

$\mathrm{m}$. For resin addition to C-636 connect a polyethylene line from the GN installed in L11 position in Cubicle 6 to the right side of a 2-line polyethylene block at HCV-C636-1 position on RR-6.

n. A floor filter assembly that consists of a standard equipment rack filter unit mounted in a filter holder made to sit on the floor. There are no available spare filter holders, but the filter holders are typically very reliable. There are currently two in service in the Hot Cell Bank. The standard equipment rack style filters can be rebuilt with new glass, gaskets, and O-rings as needed. These parts for rebuilding the filters are readily available.

o. 1-line polyethylene block at SA-F115 position-Fabricated in house. Typically spares are available.

p. Polyethylene tubing-For vacuum lines, pressure lines, demineralized water lines, and portions of the transfer route. Male ball joints and plastic compression fittings are readily available. (Previously discussed in Section

\subsection{1.q.)}

3.2.2 An up flow test of C-636 is performed as needed to confirm that C-636 has no flow restrictions before resin loading. The flow route is set to supply water from 
Tank M-63 in the MUA through C-636 into F-115 waste collection tank as in Section 3.2.1.

3.2.3 The actual resin loading utilizes the same equipment mentioned in Section 3.2.1 above, except the inlet and outlet are switched to load new resin into the column.

3.3 Pretreatment and Filtration of Feed (Product) Solution

NOTE: Evaporator Tank T-43 is used to evaporate the CLEANEX actinide product before transferring/filtering to Tank T-65 where the feed is evaporated for the final feed adjustment and pretreatment. Tank T-43 is discussed in Sections

\subsubsection{1 and 2.3.12.}

3.3.1 The feed solution for the anion exchange operations is evaporated in Tank T-43 for feed adjustment. Refer to Sections 2.3.11 and 2.3.12 for discussion of T-43 evaporator.

3.3.2 M-704/M-706tTanks [used for the addition of $\mathrm{HCl}$ and lithium chloride ( $\mathrm{LiCl}$ )] to T-43 and T-65 - Refer to Section 1.4.2 for discussion of M-704 and to Section 2.2.3 for discussion of M-706.

3.3.3 Once the feed adjustment is completed in T-43, the feed solution is transferred/filtered to T-65 through a transfer system that is set up and pretreated with synthetic feed solution ( $12 \mathrm{M} \mathrm{LiCl}, 1 \mathrm{M} \mathrm{HCl}$ ) to condition the system. The transfer route is from T-43 to T-65 through a filter and special three-prong vacuum bottle utilizing the P-653 vacuum/pressure diaphragm pump on BR-6. The filter is mounted in a filter holder made to sit on the floor. Both the filter and special three-prong vacuum bottle sit in Cubicle 6 floor.

Cell equipment utilized:

a. Special FDV at FDV-435 position (line to right) on BR-4-Polyethylene line connected from FDV-435 (routed through the Cubicle 4/5 pass through into Cubicle 5 through the Cubicle 5/6 pass through into Cubicle 6) to the inlet of a floor filter in Cubicle 6 floor; fabricated in house. Typically spares are available.

b. Standard floor filter assembly-Polyethylene line connected from FDV-435 to the inlet of a standard equipment rack filter unit mounted in a filter holder made to sit on the floor with a polyethylene line connected from the outlet of the floor filter to the inlet of a special three-prong vacuum bottle. There are no available spare filter holders, but the filter holders are typically very reliable. There are currently two in service in the Hot Cell Bank. Standard equipment rack style filters can be rebuilt with new glass, gaskets, and Orings as needed. The parts for rebuilding filters are readily available.

c. Special three-prong glass vacuum bottle-Equipped with a specially modified fitted rubber stopper with three metal prongs that penetrate the vacuum bottle to specified depths. The shortest penetration is for a vacuum 
line connection, the next shortest penetration is the solution inlet from the polyethylene line from the floor filter outlet, and the deepest penetration is the outlet line from the vacuum bottle with a polyethylene line and PVC ball valve connected to P65301 GN on BR-6. The vacuum bottles, stopper rigs, JACOs, ball joints, and polyethylene lines are typically procured and fabricated well in advance and available as needed.

d. P65301 GN on BR-6-Used with polyethylene line and PVC ball valve connected from the outlet of the special three-prong vacuum bottle. Previously discussed in Section 2.3.10.d.

e. P-653 on BR-6-Previously discussed in Section 2.3.10.e.

f. P65302 GN on BR-6-With polyethylene line connected to special FDV at FDV-T604. Previously discussed in Section 2.3.10.f.

g. Special FDV at FDV-T604 position (line at right) on BR-6-With polyethylene line connected from P65302 GN; fabricated in house. Typically spares are available.

h. T6503 jumper-Jumper runs from FDV-T604 on BR-6 to the Cubicle 6 left DC well, has a line stub (gooseneck), and is teed to the T6509 line from RR6 . There is no spare jumper and line, and replacement of this jumper and line would be difficult in the event that it experienced failure. A new jumper and line would have to be fabricated and placed into the cubicle through a Transfer Case entry. This could take 3-4 weeks to fabricate and replace the jumper.

i. T6503 line stub-Gooseneck teed to T6503 jumper from BR-6 to Cubicle 6 left DC well with a polyethylene plug and JACO fitting installed; typically spare plugs and JACOs are available

j. T6509 line-Line runs from RR-6 and is teed to the T6503 jumper from BR6. There is no spare jumper and line, and replacement of this jumper and line would be difficult in the event that it experienced failure. A new jumper and line would have to be fabricated and placed into the cubicle through a Transfer Case entry. This could take 3-4 weeks to fabricate and replace the jumper.

k. HV-T65-2-Located in T6509 line immediately before it tees into the T6503 jumper in Cubicle 6 floor, this valve is a circle seal-type valve. The valve must have a functioning valve stem installed with no leaks. O-rings are available to refurbish leaking valve stems. Also, spare stems can be made available on somewhat short notice. A leaking valve body could present a little more significant challenge since the valve is welded in place and is integral to the T6509 line.

l. 7PDV-604-2 (Type D set to 2) on BR-6-Previously discussed in Section 2.3.10.h.

m. HCV-P604-2 position (utilizing a PVC block toggle hand valve) on BR-6Previously discussed in Section 2.3.10.i.

n. Polyethylene tubing-For vacuum lines, pressure lines, demineralized water lines, and portions of the transfer route. Male ball joints and plastic 
compression fittings are readily available. Previously discussed in Section

\subsection{1.q.}

\subsection{Anion Exchange Equipment Set Up, Check Out, and Operations}

3.4.1 M-67 is a pressure transfer tank in the MUA used for transferring solutions to T635 on RR-6 for the anion exchange operations. M-67 is also set up to transfer to T-647 on BR-6 for anion exchange operations that were previously performed using C-648 Anion Exchange Column. M-67 was originally a Penton coated tank that had been in service for greater than 30 years. It was replaced with a 4 in. diameter $\times 48$ in. flanged glass column. M-67 is a nominal 9.5 L volume tank. $\mathrm{M}-67$ is used for transferring $\mathrm{LiCl}$ and $\mathrm{HCl}$ solutions. The valves associated with M-67 are typically Hayward PVC ball valves. The ball valves sometimes develop leaks, but typically they are very reliable. Normally, leaking valves can be repaired, but replacement is usually about as cost-effective as repairing.

The lines and valves associated with M-67 are listed below:

a. V-M67-1, vent line to VOG

b. V-M67-2, solution addition line through the funnel

c. V-M67-3, 5 psig air pressure supply line

d. V-M67-4, outlet line/drain from the bottom of the tank

e. V-M67-6, 25 psig air pressure supply line

f. V-M67-7, outlet line to a tee to FCV-M67-8 and V-M67-9 (FCV-M67-8 bypass line)

g. FCV-M67-8, flow control valve (hardly ever used flow control system)

h. V-M67-9, FCV-M67-8 bypass line

i. V-M67-11, outlet line to T-635 inlet on RR-6

j. V-M67-12, outlet line to T-647 inlet on BR-6

k. V-M67-15, inlet line to FCV-M67-8

l. V-M67-16, outlet line from FCV-M67-8

m. V-M67-17, outlet line from FCV-M67-8

\subsubsection{Anion Exchange Operations Feed Tank, Evaporator T-65}

T-65 has several systems that are required to operate properly to maintain functionality. Typically, tank pit tanks like T-65 do not have many physical or mechanical issues that interrupt routine operations. Loss of steam and cooling water capabilities could have a negative impact, but this is a low probability.

a. T-65-A nominal 22 L HC shell with Ta liner evaporator tank located in Tank Pit 6. T-65 has an annulus filled with Hg for increased heat transfer capability and has Ta process and instrument lines. T-65 is used to evaporate the dissolved actinide product from the target rods as part of the hot feed adjustment for anion exchange operations.

b. Foxboro pneumatic instrument-T-65's liquid level and density are read out and recorded on an original Foxboro pneumatic instrument (over 40 years 
old). This instrument is typically reliable and is routinely calibrated. T-65 pressure is read out on an original Foxboro pneumatic instrument (also over 40 years old) that provides read outs for several tank pressures. Specific tank pressures provided on this instrument are selected for read out by a selector switch. Broken polyethylene lines supplying the pneumatic signals to the transmitters and instrument can present some problems, but in recent years, UV-resistant polyethylene has decreased potential breaking of lines due to age and exposure to UV light. Plugged lines can be a problem if the plug cannot be removed easily.

c. T-65 sparger-An electric switch activates a solenoid that opens and allows air to flow into a deep leg in the tank for solution mixing in the tank. The air flow into the tank is regulated by an orifice plate in the sparge line. The orifice plate can plug requiring the suspension of sparging operations in T65. The orifice plate would have to be removed and cleaned. This typically only requires no more than $4 \mathrm{~h}$. Plugs in the sparge line downstream of the orifice or flow meter can occasionally occur; this can sometimes require as many as 12-24 h to clear. The sparger solenoid has failed, even though failure is not frequent. Rebuilds and replacements are readily available and cause very minimal down time for repairs. If necessary an alternate method of sparging T-65 is by using the in-cell air and applying air pressure down the T-65 sample uptake line.

d. T-65 processing lines-These are the Ta lines that are used to transfer process solutions into and out of T-65. Typically, these lines are not an issue, though at times some lines are preferred over others, and plugged lines can be a problem if the plug cannot be removed easily or an alternate line cannot be used.

e. T-65 sampler-A 1 L glass bottle with a 2-line rubber stopper for sampling in Cubicle 6 is connected to a 1-line polyethylene block installed in the T-65 needle block position (SA-T65) on BR-6 using polyethylene tubing. Glass bottles, stoppers, polyethylene tubing with female ball joints, polyethylene tubing, and plastic compression fittings are kept in stock and typically readily available. (The designed sample technique is no longer employed because there are no needle blocks available to use the vacuum accumulators to pull samples from the tank pit tanks.)

\subsubsection{T-65 Heating/Cooling}

a. Thermocouples-For monitoring the temperature during evaporation and feed adjustment. The thermocouples come off the tank in the tank pit and then run through the LAA wall and eventually go to the Control Room, where they read out on temperature instruments. The thermocouples are typically very reliable, but thermocouple failure necessitating thermocouple replacement is very difficult and time-consuming. Instrument failure could potentially cause significant delays as well. Thermocouple or temperature instrument failure would prevent use of T-65 as an evaporator. 
b. T-65 steam jacket and associated steam and condensate lines and valvesUsed for heating the tank for evaporation. If the steam supply to the evaporator is shut down for any reason, T-65 cannot be evaporated. Manual valves that allow RCW or steam to flow through the heating/cooling jacket on T-65 are located in the MUA. There is also a solenoid valve on the condensate discharge from T-65 that is operated by an electrical switch in the Control Room. Typically, replacement parts are available for theses valves, and most repairs are accomplished in less than $4 \mathrm{~h}$.

c. PIC-T65-The steam supply to T-65 is controlled at the main panel board in the Control Room by pressure indicator controller (PIC-T65), which supplies air pressure to a steam regulator, pressure control valve (PCV-T65), on the steam supply line to the T-65 steam jacket. Typically, the PICs are very reliable and rarely need repair or replacement. The PCVs can sometimes have diaphragm failures and leaks that require repair and occasional replacement. This can take up to $24 \mathrm{~h}$ depending on the extent of the repair.

d. T-65 RCW lines and valves-Used for cooling the tank. The RCW system supplies cooling water to T-65. Manual valves that allow RCW or steam to flow through the heating/cooling jacket on T-65 are located in the MUA. Typically, replacement parts are available for these valves, and most repairs are accomplished in less than $4 \mathrm{~h}$.

e. Condenser on the VOG line-The H-65 condenser is located in the tank pit and has RCW supplied to it for condensing the vapor in the condensate line. The RCW supply to H-65 is controlled by manual valves located in the MUA. The operability of the condenser is very reliable. Spare parts are readily available for these valves, and most repairs are accomplished in less than $4 \mathrm{~h}$.

f. VOG-Used for ventilating the tank During evaporation, vapor travels through condensers and the vapor is condensed and collected in a condensation collection tank. This line is intact, and no known reliability issues exist.

3.4.4 During the anion exchange operations, C-636 is heated to $60-70{ }^{\circ} \mathrm{C}$ using the P69 heating water loop. The P-69 heating water loop is discussed below:

a. P-69 pump-This pump has never been replaced. It has been reliable, but its age is greater than 40 years old. Procuring a replacement has not been explored. Another comparable pump could be scavenged from one of the other heating loops if necessary to avoid a significant time delay. Procuring and installing an acceptable replacement might require a significant amount of time (as much as 2-3 weeks or longer).

b. Valves V-P69-1, V-P69-2, V-P69-5, V-P69-6, V-P69-7, V-C636-3, V-C636-4, V-C648-3, V- C648-4, V-C56B-14, V-C56B -15, V-C56C-14, VC56B-15, and PRV-P69-8-Part of the P-69 heating loop system. All the valves appear to be functional as observed from the last use in late 2012. All valves but one are a combination of gate and ball valves from various 
manufacturers. Equivalent replacements for these valves are kept on hand. Typically, these do not experience instantaneous complete failure. They usually start leaking and the leak gets worse to the point that it is desirable to change the valve. A normal valve change out would typically be a $2-4 \mathrm{~h}$ job, depending on whether or not the loop was being used just prior to the change out and had to cool down before being worked on.

c. H-66 heater-This heater has not been replaced in the last 40 years. It has been reliable, but its age could be factor. Procuring a replacement has not been explored. Another comparable heater may not be available to be scavenged from one of the other heating loops, if necessary to avoid a significant time delay. The lead time for procuring an acceptable replacement could be significant.

d. TC-T66 temperature controller for the H-66/P-69 heating loop-This heating loop has been in service for over 40 years with little or no problems experienced in all that time. This is an older technology, but very reliable. The heat is controlled by a Variac controller. At this point, failure of the controller could occur the next time it is used. There may be another controller that could be scavenged to keep it working, but the probability is unlikely. A new more modern temperature controller would most likely need to be specified and a new system purchased and installed.

e. FI-P69-This flow indicator has been reliable. It has required very little or no maintenance for over 40 years of service. It is unlikely that a total failure of the flow indicator would occur. Currently, there are no exact spares on hand, but acceptable replacements could be procured and installed relatively quickly.

f. H-66 thermocouples-These are off the shelf Type K thermocouples. Failure of any of these thermocouples can be instantaneous with no warning or a gradual failure with some indication that the thermocouple is about to fail. These thermocouples are somewhat critical during the anion exchange column operations.

3.4.5 Most of the RR-6 equipment is utilized for the anion exchange operations. During the anion exchange operations, solutions are added to Tank T-635 on RR6 and pumped through the anion exchange column, C-636, using P-638 pump. The anion column effluent is collected in several different tanks by directing the column discharge through the operation of specific valves. Some of these collection tanks are located on RR-6 while others are located in Tank Pit 6 and Tank Pit 7.

Cell equipment utilized:

a. HCV-T635-2 position (utilizing a PVC block toggle hand valve) on RR-6Fabricated in house. Typically spares are available.

b. T-635-A 3 in. diameter $\times 3 \mathrm{ft}$ tall flanged glass column located on RR-6. Currently, the glass has browned because of long-term radiation exposure, and it is difficult to see a liquid level in the tank, especially at the lower 
volume which is the most critical point to be able to see the liquid level. Also, the calibration scale, once adhered to the outside of the tank has degraded and is no longer present. This old glass needs to be replaced with a new glass column with new calibration scale. This requires removal of the tank from RR-6, removal from Cubicle 6 via the Transfer Case, moving the tank to the Decontamination (Decon) Box for replacement of the glass column, replacement of the glass, returning the tank to Cubicle 6 , and reinstalling into position on RR-6. This can require as many as 3-5 working days to accomplish once a new glass is available and calibrated. Total time for repair and replacement is $\sim 7$ working days. In the past, very minor leaks around the flange gaskets, typically seen under vacuum, have occurred. Some success has been achieved by tightening the flange bolts that can be accessed while T-635 is still in position on RR-6. If the leaks cannot be stopped, then removal of T-635 to the Decon Box for repair is typically required. Spare components are usually readily available or quickly procured for repairs to T635.

c. P-638 vacuum/pressure diaphragm pump on RR-6 (referred to as a TRU pump because it was designed specifically for use in this facility) — No real spares available. P-638 has an adjustable air pressure supply (PIC-T638) that aids in controlling the flow rate. The physical pump is a standard TRU pump. Pumps can be rebuilt with significant effort. There are several nonfunctioning spare pumps available for rebuilding. Current Hot Cell Operations personnel have little or no experience in rebuilding these types of pumps. Spare parts to rebuild the pumps are limited at this writing.

d. HCV-C636-1 position (utilizing a PVC block toggle hand valve) on RR-6Fabricated in house. Typically spares are available.

e. Anion exchange column C-636 on RR-6-A nominal 1.4 L anion exchange column. C-636 is a 2 in. O.D. diameter $\times 30$ in. tall $\mathrm{Zr}$ column with a $\mathrm{Zr}$ heating/cooling jacket. It has a nominal $1.4 \mathrm{~L}$ volume. It has a metal frit at the bottom that allows the column to hold resin for performing the anion exchange operations. Refer to Section 3.2.1.h for discussion of C-636.

f. FDV-636 position (set left to right) on RR-6-Fabricated in house. Typically spares are available.

g. RE-C636-2 position-Equipped with a functioning alpha probe. There are no spare radiation element (RE) holders. Alpha probes are fabricated in house. Typically spares are available. Refer to Section 3.4.9.a for additional discussion of the alpha probe.

h. HCV-T61 position (utilizing a PVC block toggle hand valve) on RR-6Fabricated in house. Typically spares are available.

i. HCV-T630 position (utilizing a PVC block toggle hand valve) on RR-6Fabricated in house. Typically spares are available.

j. HCV-T631 position (utilizing a PVC block toggle hand valve) on RR-6Fabricated in house. Typically spares are available. 
k. HCV-T632 position (utilizing a PVC block toggle hand valve) on RR-6Fabricated in house. Typically spares are available.

l. HCV-T633 position (utilizing a PVC block toggle hand valve) on RR-6Fabricated in house. Typically spares are available.

m. HCV-T634 position (utilizing a PVC block toggle hand valve) on RR-6Fabricated in house. Typically spares are available.

n. HCV-T65 position on RR-6-Position is plugged. This position is out of service (OOS). The section of line between the C-636 discharge header and the valve position on the rack developed a leak. A special plug was fabricated and installed to isolate the leak from the header. The plug is necessary to be able to use the remainder of the header for process operations involving C-636. Also, a special polyethylene HCV block blank is installed to seal off the air line to the HCV position. The special plug and special HCV blank are fabricated in house, spares can be made available if needed.

o. HV-T635-1 position (utilizing a PVC block toggle hand valve) on RR-6Fabricated in house. Typically spares are available.

p. RE-C636-1 position on RR-6-Should be equipped with a functioning $n_{f}$ probe. This is a position that is external to the column used for $n_{f}$ scans to monitor the ${ }^{252} \mathrm{Cf}$ as it moves through the column during the loading and elution operations. There are no spare RE holders for the $\mathrm{n}_{\mathrm{f}}$ probes. Currently the $\mathrm{n}_{\mathrm{f}}$ probe holder does not function without being held in place with a master-slave manipulator. Refer to Section 3.4.9.b for additional discussion of the $\mathrm{n}_{\mathrm{f}}$ probe.

q. FDV-639 position (set top to right) on RR-6-Fabricated in house. Typically spares are available

r. HCV-T635-2 position (utilizing a PVC block toggle hand valve) on RR-6Fabricated in house. Typically spares are available.

s. HCV-C636-2 position (utilizing a PVC block toggle hand valve) on RR-6Fabricated in house. Typically spares are available.

t. HCV-T635-3 position (utilizing a PVC block toggle hand valve) on RR-6Fabricated in house. Typically spares are available.

u. HV-660 position (utilizing a PVC block toggle hand valve) on RR-6Fabricated in house. Typically spares are available.

v. HV-T65-2-A circle seal-type valve located on the T6509 jumper in Cubicle 6 floor that is connected by compression fittings. The valve bodies very rarely leak, but replacement valves are readily available. Spare O-rings are readily available for rebuilding leaking valve stems.

w. Polyethylene tubing-For vacuum lines, pressure lines, demineralized water lines, and portions of the transfer route. Male ball joints and plastic compression fittings are readily available. Previously discussed in Section

\subsection{1.q.}

3.4.6 Anion exchange elution collection tanks (T-630, T-631, T-632, T-633, and T634) and knock out tank (T-660)__Located on RR-6 behind the valves, pumps, 
filters, and other equipment. These tanks, with the exception of T-660, collect the separated TRU products and fission products and each of the collection tanks has a nominal volume of 7.3 L. The knock out tank, T-660, has a nominal volume of

2.1 L. Each tank is constructed of Ta and is equipped with pneumatic liquid level probes that read out on the Master Panel Board, MB-14, in the Control Room.

Each tank has a sample line to a special needle block/modified syringe assembly for sampling, an inlet line from a common header on RR-6 for collection of solutions from the anion exchange operations, and two deep lines for solution removal. The probes have a slight probability of plugging during operations, which could create difficulty in the confidence of the actual volume in the tank, but typically other methods can be employed to ensure that any of the tanks will not be overfilled. There have been no significant problems with these tanks than the aforementioned infrequent plugging of the liquid level probes.

\subsubsection{Anion Exchange Elution Collection Tank, Tank T-61}

T-61 has several systems that are required to operate properly to maintain functionality. Typically, tank pit tanks like T-61 do not have many physical or mechanical issues that interrupt routine operations. Plugging instrument probes or broken instrument lines can pose some minor problems, but these problems are a low probability.

a. Tank T-61-A nominal 67.5 L tank constructed of $\mathrm{Zr}$ located in Tank Pit 6 and is typically used to collect solutions from anion exchange operations in Cubicle 6.

b. Foxboro pneumatic instrument-T-61 liquid level, density, and pressure are read out and recorded on an original Foxboro pneumatic instrument (over 40 years old). This instrument is typically reliable and is routinely calibrated. Broken polyethylene lines supplying the pneumatic signals to the transmitters and instrument can present some problems, but in recent years, UV-resistant polyethylene has decreased potential breaking of lines due to age and exposure to UV light. Plugged lines can be a problem if the plug cannot be removed easily.

c. T-61 sparger-An electric switch activates a solenoid that opens and allows air to flow into a deep leg in the tank for solution mixing in the tank. The air flow into the tank is regulated by an orifice plate in the sparge line. The orifice plate can plug requiring the suspension of sparging operations in T61. The orifice plate would have to be removed and cleaned. This typically only requires no more than $4 \mathrm{~h}$. Plugs in the sparge line downstream of the orifice or flow meter can occasionally occur; this can sometimes require as many as $12-24 \mathrm{~h}$ to clear. The sparger solenoid has failed, even though failure is not frequent. Rebuilds and replacements are readily available and cause very minimal down time for repairs. If necessary an alternate method of sparging T-61 is by using the in-cell air and applying air pressure down the T-61 sample uptake line. 
d. T-61 processing lines-These are the lines that are used to transfer process solutions into and out of T-61. Typically, these lines are not an issue, though at times some lines are preferred over others, and plugged lines can be a problem if the plug cannot be removed easily or an alternate line cannot be used.

e. T-61 sampler - a $1 \mathrm{~L}$ glass bottle with a 2-line rubber stopper for sampling in Cubicle 6 is connected to a 1-line polyethylene block installed in the T-61 needle block position (SA-T61) on BR-6 using polyethylene tubing. Glass bottles, stoppers, polyethylene tubing with female ball joints, polyethylene tubing, and plastic compression fittings are kept in stock and typically readily available. (The designed sample technique is no longer employed because there are no needle blocks available to use the vacuum accumulators to pull samples from the tank pit tanks.)

\subsubsection{Anion Exchange Elution Collection Tank, Tank T-72}

Refer to Sections 1.6.2 and 1.6.3 for a complete discussion of Tank T-72.

3.4.9 The timing of making the switches to the correct collection tank is determined by alpha instrument reading, neutron instrument reading from neutron scan of C-636, volume of throughput of a particular eluting solution, and various combinations of these.

a. Alpha instrumentation consists of in-cell alpha probe at RE-C636-2 position, instrument leads and connectors from Cubicle 6 to the cell face of Cubicle 6 in the Control Room, and instrument read out and chart recorded in the Control Room. The alpha probes are fabricated in house. Historically, spare components have been available for fabricating the probes. The instrument read out and recorder was replaced in 2015 with a single new instrument that records and reads out both the alpha and neutron signals. Replacements and spare parts should be readily available for repairs.

b. Neutron instrumentation consists of in-cell $\mathrm{n}_{\mathrm{f}}$ probe used to scan C-636 to follow ${ }^{252} \mathrm{Cf}$ peak as it is loaded and eluted off the column, instrument leads and connectors from Cubicle 6 to the cell face of Cubicle 6 in the Control Room, and instrument read out and chart recorded in the Control Room. The neutron probes are fabricated in house. Historically, spare components have been available for fabricating the probes. The instrument read out and recorder was replaced in 2015 with a new single instrument that records and reads out both the alpha and neutron signals. Replacements and spare parts should be readily available for repairs.

c. Materials for fabrication of jumpers and connectors are typically readily available with spare jumpers and connectors available for use.

d. The drop leads that run into the cubicle from the cubicle ceiling have been known to fail after several years of use. Replacements are fabricated in house, and replacement requires accessing the top of the cubicle by removing 
the cubicle shield blocks. This requires a crane crew and an electrician and can take up to 1 week to complete.

3.5 Purification of Transcurium Actinides by HYDROXIDE Precipitation

NOTE: The precipitation operations utilize the RR-6 tank, Tank T-632, for precipitation and a medium porosity (nominal 10-15 $\mu \mathrm{m}$ ) glass frit filter housed in a special holder for collecting the transcurium precipitate. The filtrate is collected in Tank T-65. The precipitate is then dissolved using small amounts of $8 \mathrm{M}$ and $16 \mathrm{M} \mathrm{HNO}$ additions directly into the glass frit filter and transferring a small amount of $0.2 \mathrm{M} \mathrm{HNO}_{3}$ from T-632 through the glass frit filter into a special glass product collection bottle.

3.5.1 M-704 and M-706 are MUA tanks [used for the addition of iron (III) chloride $\left(\mathrm{FeCl}_{3}\right)$ and $\mathrm{HNO}_{3}$ solutions]. Refer to Section 1.4.2 for discussion of M-704 and to Section 2.2.3 for discussion of M-706.

3.5.2 T-632 typically contains the transcurium product and is used for precipitation of the transcurium product. Refer to Section 3.4.6 for a discussion of T-632.

3.5.3 Cold chemical additions to T-632 are made through V-M706-6 in the MUA. These chemical additions utilize the following cell equipment:

Cell equipment utilized:

a. HV-639 position (utilizing a PVC block toggle hand valve) on RR-6Fabricated in house. Typically spares are available

b. FDV-639 position (set top to right) on RR-6-Fabricated in house. Typically spares are available

c. Polyethylene line from T63905 GN to T63207 GN-Polyethylene tubing is typically readily available

3.5.4 The transfer route used for the precipitation operations is from T-632 to the glass frit filter to the filtrate collection bottle, where it is monitored with an in-cell neutron probe, then pumped out to T-65 using pump P-6053.

Cell equipment utilized:

a. MDV-637 position (set to 2) on RR-6-Fabricated in house. Typically spares are available

b. HV-637 position (utilizing a PVC block toggle hand valve) on RR-6Fabricated in house. Typically spares are available

c. T63702 GN on RR-6-Standard Zr GN with polyethylene line connected to P65301 GN on BR-6.

d. Custom fabricated medium porosity glass frit filter-Mounted in special holder clamped in a floor vise. Typically spares are available. Requires $\sim 3$ week lead time for procurement.

e. 1 L PYREX or KIMAX glass bottle-For filtrate/product collection. Typically spares are available 
f. 1 L PYREX or KIMAX glass bottle-Serves as an intermediate bottle (same as filtrate/product bottle above). Typically spares are available

g. P65301 GN on BR-6-With polyethylene line and PVC ball valve connected from the outlet of the special three-prong vacuum bottle. Previously discussed in Section 2.3.10.d.

h. P-653 on BR-6-Previously discussed in Section 2.3.10.e.

i. P65302 GN on BR-6-Used with a polyethylene line connected to special FDV at FDV-T604. Previously discussed in Section 2.3.10.f.

j. Special FDV at FDV-T604 position (line at right) on BR-6-Previously discussed in Section 3.3.3.g.

k. T6503 jumper-Previously discussed in Section 3.3.3.h.

l. T6503 line stub-Previously discussed in Section 3.3.3.i.

m. T6509 line-Previously discussed in Section 3.3.3.j.

n. HV-T65-2-Previously discussed in Section 3.3.3.k.

o. 7PDV-604-2 (Type D set to 2) on BR-6-Previously discussed in Section 2.3.10.h.

p. HCV-P604-2 position (utilizing a PVC block toggle hand valve) on BR-6Previously discussed in Section 2.3.10.i.

q. Polyethylene tubing-For vacuum lines, pressure lines, demineralized water lines, and portions of the transfer route. Male ball joints and plastic compression fittings are readily available. Previously discussed in Section

\subsection{1.q.}

3.5.5 T-65 receives the filtrate from the precipitation operations. Refer to Sections 3.4.2 and 3.4.3 for discussion of Tank T-65.

\subsection{Disposition of Actinide Product (Cm Product Cuts) from RR-6 Tanks}

3.6.1 The Cm product solution is collected in the tankage on RR-6 during the anion exchange operations.

3.6.2 The Cm product solution is transferred and flushed from the RR-6 tanks to T-40 per the following route:

Cell equipment utilized:

a. MDV-637 (set to position required for the specific tank being transferred to T-40) on RR-6- fabricated in house. Typically spares are available.

b. HV-637 position with a PVC block toggle hand valve on RR-6-Fabricated in house. Typically spares are available.

c. T63702 GN on RR-6-Previously discussed in Section 3.5.4.c.

d. P65301 GN on BR-6-Previously discussed in Section 2.3.10.d.

e. P-653 on BR-6-Previously discussed in Section 2.3.10.e.

f. P65302 GN on BR-6 - Standard Zr GN with PVC valve and polyethylene line connected to C4C601 GN in Cubicle 6 left DC well; fabricated in house. Typically spares are available. 
g. C4C601 GN in Cubicle 6 left DC well -with polyethylene line connected from P65302 GN on BR-6. Previously discussed in Section 2.3.2.h.

h. C4C601 jumper-Previously discussed in Section 2.3.2.i.

i. C4C601 jumper-Previously discussed in Section 2.3.2.j.

j. C4C601 GN on BR-4-Standard Zr GN with polyethylene line connected to T4003 GN in Cubicle 4 left DC well. (Previously discussed in Section

2.3.2.k.

k. T4003 GN_Located in Cubicle 4 left DC well (teed with T4013 line in Cubicle 6 left DC well), standard Zr GN. Typically, spares are readily available.

l. T4013GN in Cubicle 6 left DC well-T4013 line tees to T4003 line in Cubicle 4 left DC well, has a male plug installed at the DC position in the well.

m. 7PDV-604-2 (Type D set to 2) on BR-6- - Previously described in Section 2.3.10.h above.

n. 2-line polyethylene block at HCV-P604-2 position on BR-6-With a PVC valve and polyethylene line connecting the two lines. Previously described in Section 2.3.10.i above.

o. Polyethylene tubing-For vacuum lines, pressure lines, demineralized water lines, and portions of the transfer route. Male ball joints and plastic compression fittings are readily available. Previously discussed in Section

\subsection{1.q.}

\subsection{Cation Exchange Operations}

The cation exchange operations are performed utilizing equipment located on RR-4 in Cubicle 4. The feed solution is adjusted and divided into batches based on the resin loading capability of the C-402 Loading Column. The feed solution is loaded onto C-402, and then the transcurium products are eluted off (separated) through the C-401 Elution Column and collected into 250-ml polyethylene "cut" bottles. The "cut" determination is based on a combination of volumetric throughput, real-time radiological data, or a combination of data provided by in-line alpha instrumentation and neutron scans of C-401 and C-402. The "cuts" are sampled at the end of each batch of feed processed to determine the effectiveness of the separation.

\subsection{Chemical Make Ups}

NOTE: Alpha-hydroxyisobuteric acid (AHIB) or commonly referred to as "buterate" solution is the main chemical used for the cation exchange operations.

4.1.1 Nominal 25 L polyethylene bottles-All the AHIB make ups for the cation exchange operations are made up in nominal $25 \mathrm{~L}$ polyethylene bottles (and dispensed into $1 \mathrm{~L}$ polyethylene bottles then bagged into the hot cell bank as needed. 
4.1.2 Ammonium nitrate $\left(\mathrm{NH}_{4} \mathrm{NO}_{3}\right)$ - Used in a smaller quantity and made up in $1 \mathrm{~L}$ batches and poured up into $1 \mathrm{~L}$ polyethylene bottles and bagged into the hot cell bank as needed.

4.1.3 Ammonium hydroxide $\left(\mathrm{NH}_{4} \mathrm{OH}\right)$ - Used in a smaller quantity to adjust the $\mathrm{pH}$ of the AHIB make ups as needed.

4.2 Loading Column C-402 Resin Removal and Resin Addition

NOTE: Typically, the Loading Column C-402 resin is removed following the last cation exchange operation in a campaign, so that the C-402 is initially empty prior to the first run of a campaign. The C-402 resin is changed out several times during a normal campaign due to radiological and chemical damage during each batch process.

4.2.1 The route used for unloading the resin from C-402 is described below:

NOTE: All the valves located in the hot cell cubicle that are used in the cation exchanges operations are Hoke ${ }^{\circledR}$ brand ball valves that are modified for use on RR-4 and pressure tested at $>2,000$ psig prior to use.

Cell equipment utilized:

a. Loading Column C-402 on RR-4-a 5/8 in. O.D. × 8-3/4 in. long stainless steel tube with a stainless steel frit at the bottom for retaining resin and a top compression fitting that connects to a $1 / 4$ in. tubing inlet for the column. To remove the "spent" resin from the column the bottom fitting is removed and once the resin removal is complete, the bottom fitting is reconnected for the resin addition. This column has been replaced at least once in the last 20 years due to leaking at the bottom fitting. This was probably a result of an improper removal and reconnection of the fitting. A spare column may be available. C-402 can be replaced in approximately $8 \mathrm{~h}$.

b. P-407on RR-4-A positive displacement, high pressure pump that is operated by an electric motor coupled to the pump drive. Exact replacements for the motor and pump are available. Other equivalent pumps and motors may be available. Modifications of equivalent pumps for installation on RR-4 may be required.

c. $1 / 4$ in. polyethylene tubing with male ball joint on at least one endConnected to female ball joint at V-1 on RR-4, long enough to reach the cubicle floor. Typically, an ample supply of these polyethylene lines is available.

d. V-1, V-2, V-3, V-4, V-5, V-6, V-7, V-8, V-9, V-10, V-11, and V-12 on RR$4-$ Hoke ${ }^{\circledR}$ ball valves procured and then modified in house. Typically spares are available.

e. PI-407 on RR-4-Pressure gauge (range 0-2,000 psig), spare gauges with appropriate range are readily available. 
f. RE-401 position on RR-4-Holder for an alpha detector instrument, but utilizes an alpha detector pass through during resin removal. Typically, these are available in the hot cell bank. The O-ring seal may have to be replaced if the pass through leaks. Spare O-rings are available and replacement can be done in $2 \mathrm{~h}$ or less.

g. Column wrench, a 1 in. open end wrench for the $5 / 8$ in. Swagelock ${ }^{\circledR}$ fitting on the bottom of the column.

h. Miscellaneous equipment and supplies such as funnels, polyethylene bottles, polyethylene tubing, nitrogen cylinders, bottle holders, etc. are typically available.

4.2.2 Resin used for the Loading Column C-402 (Dowex 50W-X8) is typically preclassified and washed with $\mathrm{HNO}_{3}$. The pre-classified and washed resin is premeasured into a $250 \mathrm{ml}$ polyethylene bottle and transferred into the cell bank for loading into C-402.

4.2.3 The route used for loading resin into C-402 is described below:

Cell equipment utilized:

a. Loading Column C-402 on RR-4-Previously discussed in Section 4.2.1.a above.

b. Tank T-403 on RR-4-A $275 \mathrm{ml}$ volume stainless steel tank on RR-4 with a small funnel used for resin addition. It is possible to replace T-403 if it suffers catastrophic failure. It may require removal of the entire rack from its position in Cubicle 4, but it may not require the rack be removed from Cubicle 4. It would require 1-2 weeks at a minimum to fabricate the replacement tank and perform the replacement. There are no spares for $\mathrm{T}$ 403.

c. $1 / 4$ in. polyethylene tubing with male ball joint on at least one end connected to female ball joint at $\mathrm{V}-1$ on RR-4, long enough to reach the cubicle floor. Previously described in Section 4.2.1.c above.

d. V-1, V-2, V-3, V-4, V-5, V-6, V-7, V-8, V-9, V-10, V-11, and V-12 on RR$4-$ Hoke ${ }^{\circledR}$ ball valves. Previously described in Section 4.2.1.d above.

e. P-407 on RR-4-Previously discussed in Section 4.2.1.b above.

f. PI-407on RR-4-Previously discussed in Section 4.2.1.e above.

g. Tank T-404 is a 1,000 ml volume stainless steel tank on RR-4. T-404 can be replaced if it suffers catastrophic failure. It may require removal of the entire rack from its position in Cubicle 4, but it may not require the rack be removed from Cubicle 4 . It would require 1-2 weeks at a minimum to fabricate the replacement tank and perform the replacement. There are no spares for T-404.

h. PI-T404 on RR-4-Pressure gauge (-0-30 in. water vacuum and- $0-$ 100 psig pressure) for T-404 located on RR-4.

i. $\quad \mathrm{V}-13, \mathrm{~V}-14, \mathrm{~V}-15, \mathrm{~V}-16$, and V-17 on RR-4-Hoke ${ }^{\circledR}$ ball valves procured and then modified in house. Typically spares are available. 
j. Cubicle 4 Air Jet (CU4AJ) — Cubicle 4 vacuum is supplied by this air jet that is fabricated in house. Typically spares are available, but it could require up to 8 to $16 \mathrm{~h}$ to replace it.

Control room equipment utilized:

k. PIC-T404, pressure indicator controller on MB-23 in the Control Room, has experienced an occasional infrequent failure. Typically spares are available and replacement time is about $4 \mathrm{~h}$.

MUA Equipment utilized:

l. PCV-T404-The pressure control valve that is operated from PIC-T404. Supplies nitrogen pressure to T-404. Typically, it is relatively simple to repair and place back into service. Spare components are also typically available if repairs do not fix valve.

m. PI-T404-2 on RR-4-Pressure gauge (range 0-2,000 psig), spare gauges with appropriate range are readily available.

n. V-T408-1, V-T408-2, V-T408-3, V-T404-1, V-T404-2, V-T404-3, and VT404-4; bonnet needle valves. Typically spares are available.

o. Miscellaneous equipment and supplies such as funnels, polyethylene bottles, polyethylene tubing, nitrogen cylinders, bottle holders, etc. are typically available. Previously discussed in Reference 4.2.1.h above.

\subsection{Elution Column C-401 Resin Removal and Resin Addition}

NOTE: Typically, the Elution Column C-401 resin is removed following the last cation exchange operation in a campaign, so that the C-401 is initially empty prior to the first run of a campaign. The C-401 resin is changed out several times during a normal campaign due to radiological and chemical damage during each batch process.

4.3.1 The route used for unloading the resin from C-401 is described below:

NOTE: All the valves located in the hot cell cubicle that are used in the cation exchanges operations are Hoke ${ }^{\circledR}$ brand ball valves that are modified for use on RR-4 and pressure tested at $>2000$ psig prior to use.

Cell equipment utilized:

a. Elutions Column C-401on RR-4-a 1 in. O.D. $\times 44$ in. long stainless steel outer tube with a 5/8 in. O.D. $\times$ 47-9/16 in. long inner tube with a stainless steel frit at the bottom for retaining resin and a top compression fitting that connects to a $1 / 4 \mathrm{in}$. tubing inlet for the column. The column has a water jacket connected to a heating/cooling water loop that supplies cooling and heating water between the outer and inner tubes. To remove the "spent" resin from the column the bottom fitting is removed and once the resin removal is complete, the bottom fitting is reconnected for the resin addition. A spare column is not available. C-401 can be replaced in approximately $8 \mathrm{~h}$. 
b. V-1, V-2, V-3, V-4, V-5, V-6, V-7, V-8, V-9, V-10, V-11, and V-12Hoke ${ }^{\circledR}$ ball valves on RR-4. Previously described in Section 4.2.1.d above.

c. $1 / 4 \mathrm{in}$. polyethylene tubing with male ball joint on at least one end connected to female ball joint at $\mathrm{V}-1$ on RR-4, long enough to reach the cubicle floor. Typically, an ample supply of these polyethylene lines is available.

d. Pump P-407 on RR-4—Previously described in Section 4.2.1.b above.

e. PI-407 on RR-4-Previously described in Section 4.2.1.e above.

f. RE-401 on RR-4-Previously described in Section 4.2.1.f above.

g. Column wrenches, a 1 in. wrench for the $5 / 8$ in. Swagelock ${ }^{\circledR}$ fitting on the bottom of the column. Previously described in Section 4.2.1.g above.

h. Miscellaneous equipment and supplies such as funnels, polyethylene bottles, polyethylene tubing, nitrogen cylinders, bottle holders, etc. are typically available. Previously discussed in Section 4.2.1.h above.

4.3.2 Resin used for the Elution Column C-401 (Dowex 50W-X8) is typically preclassified and washed with $\mathrm{HNO}_{3}$. The pre-classified and washed resin is premeasured into a $250 \mathrm{ml}$ polyethylene bottle and transferred into the cell bank for loading into C-401.

4.3.3 The route used for loading resin into C-401 is described below:

Cell equipment utilized:

a. Elution Column C-401 on RR-4-Previously discussed in Section 4.3.1.a above.

b. Tank T-403on RR-4-Previously described in Section 4.2.3.b above.

c. $1 / 4$ in. polyethylene tubing with male ball joint on at least one end connected to female ball joint at $\mathrm{V}-1$ on RR-4, long enough to reach the cubicle floor. Previously described in Section 4.2.3.c above.

d. V-1, V-2, V-3, V-4, V-5, V-6, V-7, V-8, V-9, V-10, V-11, and V-12 on RR-4 Hoke ${ }^{\circledR}$ ball valves-Previously described in Section 4.2.1.d above.

e. P-407 on RR-4-Previously described in Section 4.2.1.b above.

f. PI-407 on RR-4-Previously described in Section 4.2.1.e above.

g. RE-401 on RR-4-Previously described in Section 4.2.1.f above.

h. Tank T-404 on RR-4-Previously described in Section 4.2.3.g above.

i. PI-T404 on RR-4-Previously described in Section 4.2.3.h above.

j. V-13, V-14, V-15, V-16, and V-17 on RR-4-Hoke ${ }^{\circledR}$ ball valves. Previously described in Section 4.2.3.i above.

k. CU4AJ—Cubicle 4 vacuum. Previously described in Section 4.2.3.j above.

Control room equipment utilized:

l. PIC-T404-Previously described in Section 4.2.3.k above.

MUA equipment utilized:

m. PCV-T404-Previously described in Section 4.2.3.I above.

n. PI-T404-2_Previously described in Section 4.2.3.m above. 
o. V-T408-1, V-T408-2, V-T408-3, V-T404-1, V-T404-2, V-T404-3, and VT404-4; bonnet needle valves Previously described in Section 4.2.3.n above.

p. Miscellaneous equipment and supplies such as funnels, polyethylene bottles, polyethylene tubing, nitrogen cylinders, bottle holders, etc. are typically available. (Previously discussed in Section 4.2.1.h above.)

4.3.4 Once loaded, the resin in C-401 is washed with $0.3 \mathrm{M} \mathrm{NH}_{4} \mathrm{NO}_{3}$ that is made up per Section 4.1.2 and transferred into the cell bank.

4.3.5 After the Elution Column C-401 has been treated with $0.3 \mathrm{M} \mathrm{NH}_{3} \mathrm{NO}_{3}$, both the Loading Column C-402 and the Elution Column C-401 are flushed with water.

4.4 Actinide Loading onto the Loading Column C-402

\subsubsection{H-410 Heating Loop}

a. V-H410-1, V-H410-2, V-H410-3, V-H410-4, V-H410-5, V-P460-6, HVC401-26, and PRV-H410-Part of the H-410 heating loop system. All the valves appear to be functional as observed from the last use in late 2012. All these valves, but one, are ball valves from various manufacturers and equivalent replacements for these valves are kept on hand. Typically, these do not experience instantaneous complete failure. They usually start leaking and the leak gets worse to the point that it is desirable to change the valve. The one valve not a ball or gate valve is a pressure relief valve. The valve change out would typically be a 2 to $4 \mathrm{~h}$ job, depending on whether or not the loop was being used just prior to the change out and had to cool down before being worked on.

b. H-410 heater-This heater was replaced sometime around the year 2000. It has been reliable, since its installation. Procuring a replacement has not been explored. Another comparable heater is not available to be scavenged from one of the other heating loops to avoid significant down time if failure occurs. The lead time for procuring an acceptable replacement could be significant.

c. TC-H410 temperature controller for the H-410 heating loop-This controller was replaced sometime around the year 2000 with little or no problems experienced since its installation. This controller so far has proven very reliable. A spare may not be available, but procurement of an acceptable replacement in 1-2 weeks is probable.

d. FI-H410 - This flow indicator has proven to be reliable. It has required very little or no maintenance since it was installed sometime around the year 2000. It is unlikely that a total failure of the flow indicator would occur. There may be a spare available, but if not, an acceptable replacement could be procured and installed relatively quickly.

e. H-410 Thermocouples-These are off the shelf Type K thermocouples. Failure of the thermocouples can be instantaneous with no warning or a gradual failure can occur with some indication that the thermocouple is about 
to fail. Failure of just one thermocouple may not be an issue, but failure of two thermocouples could result in suspension of the cation exchange operations in Elution Column C-401 until replacements are installed.

4.4.2 The feed solution is from the hot feed bottle into T-404 then through C-402 to load the actinide product onto the resin per the route described below:

Cell equipment utilized:

a. Tank T-404 on RR-4-Previously described in Section 4.2.3.g above.

b. $1 / 4$ in. polyethylene tubing with male ball joints on each end with one end connected to female ball joint at V-15 on RR-4, long enough to reach the cubicle floor.

c. V-1, V-2, V-3, V-4, V-5, V-6, V-7, V-8, V-9, V-10, V-11, and V-12Hoke ${ }^{\circledR}$ ball valves on RR-4. Previously described in Section 4.2.1.d above.

d. V-13, V-14, V-15, V-16, and V-17, Hoke ${ }^{\circledR}$ ball valves on RR-4-Previously described in Section 4.2.3.i above.

e. CU4AJ-Cubicle 4 Vacuum. Previously described in Section 4.2.3.j above.

f. RE-401 on RR-4-Previously described in Section 4.2.1.f above.

Control room equipment utilized:

g. PIC-T404 Previously described in Section 4.2.3.k above.

MUA equipment utilized:

h. PCV-T404-Previously described in Section 4.2.3.I above.

i. PI-T404-2-Previously described in Section 4.2.3.m above.

j. V-T408-1, V-T408-2, V-T408-3, V-T404-1, V-T404-2, V-T404-3, and V-T404-4; bonnet needle valves-Previously described in Section 4.2.3.n above.

k. 1/8 in. stainless steel dip wands used for transferring the feed solution.

l. Miscellaneous equipment and supplies such as funnels, polyethylene bottles, polyethylene tubing, nitrogen cylinders, bottle holders, etc. are typically available. Previously discussed in Reference 4.2.1.h above.

4.4.3 Once the hot feed has been transferred through C-402, a water flush of T-404 (or the hot feed bottle, if it was emptied), conditioning C-402 with $0.3 \mathrm{M} \mathrm{NH}_{4} \mathrm{NO}_{3}$ (made per Section 4.12), and a final water flush of T-404 (or the hot feed bottle, if it was emptied) are transferred through C-402 using the same route described in Section 4.4.2.

4.5 Elution and Separation of Actinides Using the Elution Column C-401

4.5.1 Once hot feed has been loaded and the flushing and conditioning of C-402 has been completed, elution of the actinides is started by pumping the prescribed AHIB solutions through C-402 and C-401 per the route below:

a. Loading Column C-402-Previously discussed in Section 4.2.1.a above. 
b. Elution Column C-401-Previously discussed in Section 4.3.1.a above.

c. $1 / 4 \mathrm{in}$. polyethylene tubing with male ball joints on each end with one end connected to female ball joint at V-15 on RR-4, long enough to reach the cubicle floor. Previously described in Section 4.4.2.b above.

d. V-1, V-2, V-3, V-4, V-5, V-6, V-7, V-8, V-9, V-10, V-11, and V-12Hoke ${ }^{\circledR}$ ball valves. Previously described in Section 4.2.1.d above.

e. Pump P-407-Previously described in Section 4.2.1.b above.

f. PI-407-Previously described in Section 4.2.1.e above.

g. RE-401-Previously described in Section 4.2.1.f above.

h. Discharge line specially fabricated from Tygon ${ }^{\circledR}$ with a stainless steel end piece bent into a hook to hook into collection bottles.

i. Approximately 25 labeled $250 \mathrm{ml}$ polyethylene bottles for collecting product cuts. These bottles are typically bought in bulk and available for use.

j. $\quad 1 / 8$ in. stainless steel dip wands $-1 / 8$ in. stainless steel tubing with a female ball joint on one end, used for transferring elution solutions, made in house. Typically spares are readily available.

k. Miscellaneous equipment and supplies such as funnels, polyethylene bottles, polyethylene tubing, nitrogen cylinders, bottle holders, etc. are typically available. Previously discussed in Reference 4.2.1.h above.

4.5.2 The product collection is determined by volume of throughput of a particular eluting solution, alpha instrument reading, neutron instrument reading from neutron scan of C-401, and various combinations of these.

a. Alpha instrumentation consists of in-cell alpha probe at RE-401 position, instrument leads and connectors from Cubicle 4 to the cell face of Cubicle 4 in the Control Room, and instrument read out and chart recorded in the Control Room. The alpha probes are fabricated in house. Historically, spare components have been available for fabricating the probes. The instrument read out and recorder was replaced in 2015 with a single new instrument that records and reads out both the alpha and neutron signals. Replacements and spare parts should be readily available for repairs.

b. Neutron instrumentation consists of in-cell $n_{f}$ probe used to scan C-401 and C-402 to follow ${ }^{252} \mathrm{Cf}$ peak as it is loaded and eluted off the columns, instrument leads and connectors from Cubicle 4 to the cell face of Cubicle 4 in the Control Room, and instrument read out and chart recorded in the Control Room. The neutron probes are fabricated in house. Historically, spare components have been available for fabricating the probes. The instrument read out and recorder was replaced in 2015 with a new single instrument that records and reads out both the alpha and neutron signals. Replacements and spare parts should be readily available for repairs.

c. Materials for fabrication of jumpers and connectors are typically readily available with spare jumpers and connectors available for use.

d. The drop leads that run into the cubicle from the cubicle ceiling have been known to fail after several years of use. Replacements are fabricated in 
house, and replacement requires accessing the top of the cubicle by removing the cubicle shield blocks. This requires a crane crew and an electrician and can take up to 1 week to complete.

4.6 Acidification and Sampling of Product Cuts and Raffinate Solutions

4.6.1 Concentrated $\mathrm{HNO}_{3}$ - The product cuts and raffinates are acidified using concentrated $\mathrm{HNO}_{3}$ bagged into the hot cell and dispensed based on the volume of each cut. The acid is typically dispensed using a small volume pipette and rubber bulb.

4.6.2 Sample thief pipettes-Each cut and raffinate is mixed and sampled using a small glass sample thief (small volume pipette) and small rubber bulb, and then the samples are submitted for analysis. An ample supply of sample thieves and rubber bulbs are kept on hand for use.

4.7 Cation Exchange Operation Follow Up and Product Compositing

4.7.1 C-402 Loading Column resin is typically dumped at the conclusion of the series of cation exchanges operations, leaving C-402 empty. New resin is not added back to C-402. Refer to Section 4.2.

4.7.2 C-401 Elution Column resin is typically dumped at the conclusion of the series of cation exchanges operations, leaving C-401 empty. New resin is not added back to C-401. Refer to Section 4.3.

4.7.3 The ${ }^{252} \mathrm{Cf}$ product cuts are composited into a $2 \mathrm{~L}$ polyethylene bottle and held for loading onto Pt capsules. The compositing consists of pouring each cut bottle into the $2 \mathrm{~L}$ bottle using a funnel and flushing each bottle with a small prescribed amount of concentrated $\mathrm{HNO}_{3}$ acid and demineralized water. The bottles are probed for neutron activity to evaluate the effectiveness of the flushes. Adequate supplies of $2 \mathrm{~L}$ polyethylene bottles and funnels are available for use.

4.7.4 Recycle cuts are determined by evaluation of the sample results reported by the analytical chemistry lab. These cuts are transferred and flushed into a tank pit tank and eventually combined with other recoverable material and processed at a later time. The selected cuts are transferred via a vacuum/pressure tank (typically T-607, located on BR-6 in Cubicle 6) to T-66 or T-79 (Refer to Sections 10.2.1 and 10.2.2 for discussion of T-66 and T-79, respectively.) and eventually to T21, or T-23 (Refer to Sections 2.3.3 and 2.3.4 for discussion of T-21 and T-23, respectively.) for miscellaneous CLEANEX extraction for later recovery. The T607 transfer route is discussed in Section 9.2.8.

4.7.5 Waste solutions are also determined by evaluation of the sample results reported by the analytical chemistry lab. These cuts are transferred and flushed into a tank pit tank and eventually transferred into the waste tank. The selected cuts are transferred using a vacuum/pressure tank (typically T-607, located on BR-6 in 
Cubicle 6) to T-66 or T-79 (refer to Sections 10.2.1 and 10.2.2 for discussion of T-66 and T-79, respectively) and then on to F-115 via steam jet or the T-607 transfer system. The T-607 transfer system is discussed in Section 9.2.8.

\subsection{Californium Packaging}

The Cf package loading operations are performed utilizing equipment located on RR-4. The loading station is comprised of a feed tank, piping, and valves to the loading station and a nitrogen cylinder supplied pressure system. The feed for the package loading is composited ${ }^{252} \mathrm{Cf}$ product cuts from the cation exchange operations. Refer to Section 4.0 above.

\subsection{Preparations}

5.1.1 Platinum capsules and nut packages are fabricated in house.

a. The Pt capsules consist of a 3/8 in. Pt tube that measures $13 / 16$ in. long containing $1.0 \mathrm{ml}$ of DOWEX resin with Pt frits on each end to contain the resin in the tube and allow liquid flow through the tube. Because Pt is a precious metal, the Pt capsules are fabricated and controlled by the precious metal inventory and secured under lock and key until required for Cf loading.

b. The package (nut package) that holds the Pt capsule is comprised of two $3 / 8$ in. Swagelock nuts welded in a configuration to capture the Pt capsule. Each package is pressure tested at $>2,000$ psig before use. Typically, adequate Pt capsules and nut packages are available as needed.

5.1.2 The following transfer system is used for the loading operations.

Cell equipment utilized:

a. Tank T-408-A $~ 1,000 \mathrm{ml}$ volume stainless steel tank on RR-4. T-408 is the feed tank for the package loading operations. It may be possible to replace T408 if it suffers catastrophic failure. It may require removal of the entire rack from its position in Cubicle 4, but it may not require the rack be removed from Cubicle 4. It would require 1-2 weeks at a minimum to fabricate the replacement tank and perform the replacement. There are no spares for T408.

b. Tank T-405-A $500 \mathrm{ml}$ volume stainless steel tank on RR-4. T-405 is the tank used for making feed transfers. T-405 is evacuated with a cubicle vacuum, isolated from the cubicle vacuum, and then used as the vacuum source for transferring solutions into T-408. It may be possible to replace T405 if it suffers catastrophic failure, but replacement is unlikely. It may require removal of the entire rack from its position in Cubicle 4, but not from Cubicle 4. It would require 1-2 weeks at a minimum to fabricate the replacement tank and perform the replacement. There are no spares for T405.

c. $1 / 4$ in. polyethylene tubing - Polyethylene lines with male ball joint on at least one end connected to female ball joint at V-18 on RR-4. It is long enough to 
reach the cubicle floor. Typically, an ample supply of these polyethylene lines is available.

d. V-13, V-14, V-16, V-17, V-18, V-19, and V-20-Hoke ${ }^{\circledR}$ ball valves that are procured and then modified in house. Typically spares are available.

e. PI-T408-1-Pressure gauge for tank T-408 (range 0-30 in. Hg vacuum; 0500 psi pressure). Spare gauges with appropriate range are readily available.

f. CU4AJ-Cubicle 4 vacuum. Previously described in Section 4.2.3.j above.

Control room equipment utilized:

g. PIC T404-Previously described in Section 4.2.3.k above.

MUA equipment utilized:

h. PCV-T404-Previously described in Section 4.2.3.1 above.

i. PI-T404-2-Previously described in Section 4.2.3.m above.

j. HS-T408-4 and HS-T408-5-Push button switches that operate a pressure controller comprised of two buttons on MB-23 in the Control Room. HST408-4 increases the pressure supply to T-408, and HS-T408-5 decreases the pressure supply to T-408.

k. PCV-T408-The pressure control valve that is operated from two buttons on MB-23 in the Control Room. The PCV has experienced infrequent failure. Typically spares are available and replacement time is about $4 \mathrm{~h}$.

l. PI-T408-2-Pressure gauge (range $0-2,000$ psig). Spare gauges with appropriate range are readily available.

m. V-T408-1, V-T408-2, V-T408-3, V-T404-1, V-T404-2, V-T404-3, and VT404-4-Bonnet needle valves. Previously described in Section 4.2.3.n above.

n. Miscellaneous equipment and supplies-Funnels, polyethylene bottles, polyethylene tubing, bottle holders, etc. are typically available. Previously discussed in Reference 4.2.1.h above.

5.1.3 The hot feed transfer and package loading route are leak checked before use. Any leaking valves are replaced as needed.

\subsection{Cf Package Loading Operations}

5.2.1 A Cf package is installed into the loading position on RR-4 using a wrench modified for in-cell use. Cf packages were previously discussed in Section 5.1.1 above.

5.2.2 Additional leak testing of the loading route is performed as outlined in Section 5.1.2 above.

5.2.3 Hot feed is mixed in the $2 \mathrm{~L}$ Cf product bottle, and a prescribed amount is transferred to T-408 using the in-cell vacuum connected to T-408 via a small knock out tank, T-405, on RR-4. 
5.2.4 T-408 is pressurized, and the hot feed is transferred through the Pt capsule/nut package to load the Cf onto the resin utilizing the route set up and tested in Section 5.1.2 above.

5.2.5 Once T-408 is empty, T-408 is flushed with water through the Pt capsule/nut package.

5.2.6 The Pt capsule/nut package is allowed to vent for a prescribed period of time, then it is removed from position on RR-4.

\subsection{Cf Package Firing in the Cubicle 4 Muffle Furnace}

5.3.1 Firing wand - The Pt capsule is removed from the nut package and installed into a firing wand. The firing wand is a 1/8 in. diameter stainless steel tubing with a compression fitting for attaching the Pt capsule to it, and the opposite end has a female ball joint for attaching a purge line. The firing wand is fabricated in house. Firing wands tend to last a long time, and spare firing wands are fabricated relatively quickly if they are needed.

5.3.2 Polyethylene line-A $1 / 4$ in. diameter polyethylene line with male ball joints on each end is connected to a two-bottle bubbler system with in-cell vacuum connected to the bottle system. The vacuum is pulled on the two bottles set up in series through the firing wand to pull air through the capsule.

5.3.3 H-520 muffle furnace-The Pt capsule is placed into a Thermolyne 48010 series muffle furnace, $\mathrm{H}-520$, located in Cubicle 4 and fired in a prescribed firing sequence at prescribed temperatures. The muffle furnace currently in use does not have a spare, but two spares have been procured. These are standard off the shelf furnaces, but there is a certain amount of preparation required to make the furnace "hot cell” ready. Currently, sketches are in process of being generated for the modifications to be made. Procurement time is approximately 3-4 weeks with $\sim 1-2$ weeks of in house preparations after the sketches are completed.

5.3.4 Temperature Indicator Controller (TIC) TIC-520 furnace controller-The temperature controller for H-520 muffle furnace is a Eurotherm Model 808. This particular model is no longer available, but comparable models from Eurotherm are available. No exact replacement spares are on hand. Other manufacturers of temperature controllers also have comparable controllers that could be substituted for the current model. Lead time for procurement and installation is 1-2 weeks.

5.3.5 Nut package-Once the firing sequence is complete, the Pt capsule is removed from the firing wand and reinstalled into the nut package. Sections 5.2.1-5.2.6 and Sections 5.3.1-5.3.4 are repeated until all the Cf feed solution has been loaded onto packages. 


\subsection{Loaded Cf Packages}

The loaded Cf packages are held in one of the cubicles in Building 7920 until needed for filling Cf orders. As inventory is needed for filling orders, Cf packages are transferred from the hot cell cubicles into the Target Decontamination Facility, a portable watershielded hot cell and pneumatically transferred to Building 7930.

\subsection{HYDROXIDE Precipitation of the Actinide Product}

NOTE: The HYDROXIDE precipitation operations are performed in multiple batches, depending on the amount of radiological material to be processed; therefore, the operations are typically repeated several times for each processing campaign.

6.1 Chemical Make Ups

6.1.1 Sodium hydroxide $(\mathrm{NaOH})$ and nitric acid $\left(\mathrm{HNO}_{3}\right)$ are the two chemicals used for the HYDROXIDE precipitation operations. These chemicals are usually kept in ample supply.

6.1.2 All chemical make ups are made up and stored in polyethylene bottles. An ample supply of polyethylene bottles is readily available for use.

\subsection{Feed Adjustment}

\subsubsection{HYDROXIDE Precipitation Feed Tank, Evaporator T-60}

T-60 has several systems that are required to operate properly to maintain functionality. Typically, tank pit tanks like T-60 do not have many physical or mechanical issues that interrupt routine operations. Loss of steam and cooling water capabilities could have a negative impact, but this is a low probability.

a. T-60-A nominal 63 L HC shell with Ta liner evaporator tank located in Tank Pit 6. T-60 has an annulus filled with Hg for increased heat transfer capability and is used to evaporate the dissolved $\mathrm{Cm}$ product from the anion exchange operations.

b. Foxboro pneumatic instrument-T-60's liquid level and density are read out and recorded on an original Foxboro pneumatic instrument (over 40 years old). This instrument is typically reliable and is routinely calibrated. T-60 pressure is read out on an original Foxboro pneumatic instrument (also over 40 years old) that provides read outs for several tank pressures. Specific tank pressures provided on this instrument are selected for read out by a selector switch. Broken polyethylene lines supplying the pneumatic signals to the transmitters and instrument can present some problems, but in recent years, UV-resistant polyethylene has decreased potential breaking of lines due to age and exposure to UV light. Plugged lines can be a problem if the plug cannot be removed easily. 
c. T-60 sparger-An electric switch activates a solenoid that opens and allows air to flow into a deep leg in the tank for solution mixing in the tank. The air flow into the tank is regulated by an orifice plate in the sparge line. The orifice plate can plug requiring the suspension of sparging operations in T60 . The orifice plate would have to be removed and cleaned. This typically only requires no more than $4 \mathrm{~h}$. Plugs in the sparge line downstream of the orifice or flow meter can occasionally occur. This can sometimes require as many as 12-24 h to clear. The sparger solenoid has failed, even though failure is not frequent. Rebuilds and replacements are readily available and cause very minimal down time for repairs. If necessary an alternate method of sparging T-60 is by using the in-cell air and applying air pressure down the T-60 sample uptake line.

d. T-60 processing lines-These are the Ta lines that are used to transfer process solutions into and out of T-60. Typically, these lines are not an issue, though at times some lines are preferred over others, and plugged lines can be a problem if the plug cannot be removed easily or an alternate line cannot be used.

e. T-60 sampler-A $1 \mathrm{~L}$ glass bottle with a 2-line rubber stopper for sampling in Cubicle 6 is connected to a polyethylene line that is connected to a standard Ta GN installed in the T6002 position in the Cubicle 6 left disconnect well. The T6002 jumper has been removed because the T6002 line on the sample rack leaked. T6002 GN in Cubicle 6 is to left of the DC well. Glass bottles, stoppers, polyethylene tubing with female ball joints, polyethylene tubing, and plastic compression fittings are kept in stock and are typically readily available. (The designed sample technique is no longer employed because the T-60 vacuum accumulator and needle block position [SA-T60] are out of service.)

\subsubsection{T-60 Heating/Cooling}

a. Thermocouples-For monitoring the temperature during evaporation and feed adjustment. The thermocouples come off the tank in the tank pit and then run through the LAA wall and eventually go to the Control Room, where they read out on temperature instruments. The thermocouples are typically very reliable, but thermocouple failure necessitating replacement is very difficult and time-consuming. Instrument failure could potentially cause significant delays as well. Thermocouple or temperature instrument failure would prevent use of T-60 as an evaporator.

b. T-60 steam jacket and associated steam and condensate lines and valvesUsed for heating the tank for evaporation. If the steam supply to the evaporator is shut down for any reason, T-60 cannot be evaporated. Manual valves that allow RCW or steam to flow through the heating/cooling jacket on T-60 are located in the MUA. There is also a solenoid valve on the condensate discharge from T-60 that is operated by an electrical switch in the 
Control Room. Typically, replacement parts are available for these valves, and most repairs are accomplished in less than $4 \mathrm{~h}$.

c. PIC-T60 - The steam supply to T-60 is controlled at the main panel board in the Control Room by pressure indicator controller (PIC-T60), which supplies air pressure to a steam regulator, pressure control valve (PCV-T60), on the steam supply line to the T-60 steam jacket. Typically, the PICs are very reliable and rarely need repair or replacement. The PCVs can sometimes have diaphragm failures and leaks that require repair and occasional replacement. This can take up to $24 \mathrm{~h}$, depending on the extent of the repair.

d. T-60 RCW lines and valves-Used for cooling the tank. The RCW system supplies cooling water to T-60. Manual valves that allow RCW or steam to flow through the heating/cooling jacket on T-60 are located in the MUA. Typically, replacement parts are available for theses valves, and most repairs are accomplished in less than $4 \mathrm{~h}$.

e. Condenser on the VOG line-The H-60 condenser is located in the tank pit and has RCW supplied to it for condensing the vapor in the condensate line. The RCW supply to H-60 is controlled by manual valves located in the MUA. The operability of the condenser is very reliable. Spare parts are readily available for these valves, and most repairs are accomplished in less than $4 \mathrm{~h}$.

f. VOG-Used for ventilating the tank and during evaporation. Vapor travels through condensers, and the vapor is condensed and collected in a condensation collection tank. This line is intact, and no known reliability issues exist.

6.2.3 Additions of concentrated $\mathrm{HCl}$ to T-60 (can use either V-T60-4 to T-604 decon line or Cubicle 6 solution addition line connected to T6002 line in Cubicle 6 from the MUA using Tank M-704 Refer to Section 1.4.2 for discussion of M-704.

a. V-T60-4 (T-60 decon line)—A PVC ball valve. Typically spares are available.

b. V-CU6-1 (Cubicle 6 solution addition line) —A PVC ball valve in the MUA that goes to a standard $\mathrm{Zr}$ GN located in service lines on the east wall of Cubicle 6 with a polyethylene line connected to T6002 GN; fabricated in house. Typically spares are available.

c. T6002 GN in Cubicle 6 left DC well-Standard Zr GN with polyethylene line from Cubicle 6 solution addition line; fabricated in house. Typically spares are available.

6.2.4 Once the feed adjustment is complete, the feed is transferred to T-61 through the route below.

Cell equipment utilized:

a. T6007 GN in Cubicle 6 left DC well-With polyethylene line connected from to C4C601 GN; fabricated in house. Typically spares are available. 
b. C4C601 GN in Cubicle 6 left DC well-With polyethylene line connected from T6007 GN in Cubicle 6 left DC well. Previously described in Section 2.3.2.h.

c. C4C601 jumper-Previously described in Section 2.3.2.i.

d. C4C601 jumper-Previously described in Section 2.3.2.j.

e. C4C601 GN on BR-4-Standard Zr GN with polyethylene line connected to PVC ball valve on right side of 2-line block at PS-431 position on BR-4 in Cubicle 4. Previously discussed in Section 2.3.2.k.

f. 2-line block at PS-431 position on BR-4-Polyethylene line connected from C4C601 GN on BR-4. Fabricated in house. Typically spares are available.

g. FDV-440 position on BR-4 (set top to left)—Polyethylene FDV blocks are typically fabricated in house. Typically spares are available.

h. T-430-A 3 in. diameter $\times 3 \mathrm{ft}$ tall flanged glass column located on BR-4. Over time the glass becomes dark from radiation exposure, and there have been a few incidents where the glass has broken. This requires removal of the tank from BR-4, removal from Cubicle 4 via the Transfer Case, moving the tank to the Decon Box for replacement of the glass and gaskets, returning the tank to Cubicle 4, and reinstalling into position on BR-4. This can require as many as $3-5$ working days to accomplish. In the past, very minor leaks around the flange gaskets, typically seen under vacuum, have occurred. Some success has been achieved by tightening the flange bolts that can be accessed while T-430 is still in position on BR-4. If the leaks cannot be stopped, then removal of T-430 to the Decon Box for repair is typically required. Spare components are usually available for repairs to T-430.

i. 2-line block at HVC-T430-2 position on BR-4-With polyethylene line connected to PVC ball valve on right side of 2-line block that spans from Cubicle 4 to Cubicle 6 connected to T6107 GN. These 2-line HCV blocks are usually fabricated in house, and typically spares are available.

j. T6107 GN in Cubicle 6 left DC well—Standard Zr GN with polyethylene line connected right side of 2-line block at HCV-T430-2.

k. Cubicle 6 solution addition line GN-With polyethylene line to be connected to T-6107 GN in Cubicle 6 (as required).

l. CU4AJ-Cubicle 4 vacuum. Previously described in Section 4.2.3.j above.

m. Polyethylene tubing — For vacuum lines, pressure lines, demineralized water lines, and portions of the transfer route. Male ball joints and plastic compression fittings are readily available. Previously discussed in Section

\subsection{1.q.}

\subsubsection{HYDROXIDE Precipitation Tank, Tank T-61}

T-61 is also used in the anion exchange operations. Refer to Section 3.4.7 for discussion of Tank T-61.

6.2.6 M-421 is a nominal 5.5 L volume glass column (3 in. diameter $\times 48$ in. tall) tank. M-421 is located in the MUA and is used for solution additions to T-54, T-43, T- 
45, and the LR-4 tanks supporting the hydroxide precipitation operations. The top and bottom flanges are constructed out of stainless steel. Inlet lines into M421 come in through the top flange, and there is one outlet line exiting through the bottom flange. The inlet lines and outlet line have Jamesbury ball valves for air pressure, water and venting utilities, solution addition, and solution discharge. Typically, this type tank requires minimal maintenance, and spare parts are readily available if required. The ball valves sometimes develop leaks, but typically they are very reliable. Normally, leaking valves can be repaired, but replacement is usually about as cost-effective as repairing. The lines and valves associate with these tanks are listed below:
a. V-M421-1, vent line to VOG
b. V-M421-2, solution addition line through the funnel
c. V-M421-3, 5 psig air pressure supply line
d. V-M421-4, drain line/solution outlet
e. V-M421-7, demineralized water addition line

6.2.7 Once the feed transfer to T-61 is complete, $4 \mathrm{~L}$ of $5 \mathrm{M} \mathrm{NaOH}$ is added from $\mathrm{M}$ 421 (previously discussed in Section 6.2.6 above) in the MUA to T-61at a rate of $100 \pm 20 \mathrm{ml} / \mathrm{min}$ while sparging T-61 to precipitate the $\mathrm{Cm}$.

6.2.8 After the prescribed sparge period and settling period, the solution is filtered to T-20 using T-444.

Cell equipment utilized:

a. T6107 GN in Cubicle 6 left DC well—Standard Zr GN with polyethylene line connected to C4C601 GN; fabricated in house. Typically spares are available.

b. C4C601 GN in Cubicle 6 left DC well-With polyethylene line connected from T6107 GN in Cubicle 6 left DC well. Previously described in Section 2.3.2.h.

c. C4C601 jumper-Previously described in Section 2.3.2.i.

d. C4C601 jumper-Previously described in Section 2.3.2.j.

e. C4C601 GN on BR-4-Standard Zr GN with polyethylene line connected to PVC ball valve on right side of 2-line block at F-445 position on LR-4. Previously discussed in Section 2.3.2.k.

f. 2-line block at F-445 position on LR-4-Polyethylene line connected from C4C601 GN in Cubicle 4 to PVC ball valve on right side of 2-line block at F-445 position on BR-4 in Cubicle 4. These 2-line blocks are usually fabricated in house, and typically spares are available.

g. T-444-A 3 in. diameter $\times 3 \mathrm{ft}$ tall flanged glass column located on LR-4. Over time the glass becomes dark from radiation exposure, and there have been a few incidents where the glass has broken. This requires removal of the tank from LR-4, removal from Cubicle 4 via the Transfer Case, moving the tank to the Decon Box for replacement of the glass and gaskets, returning the 
tank to Cubicle 4, and reinstalling into position on LR-4. This can require as many as 3-5 working days to accomplish. In the past, very minor leaks around the flange gaskets, typically seen under vacuum, have occurred. Some success has been achieved by tightening the flange bolts that can be accessed while T-444 is still in position on LR-4. If the leaks cannot be stopped, then removal of T-444 to the Decon Box for repair is typically required. Spare components are usually available for repairs to T-444.

h. 2-line block at HVC-T444-2 position on RR-4-With polyethylene line connected from PVC ball valve on right side of 2-line block to inlet line to the deep bed filter. These 2-line HCV blocks are usually fabricated in house, and typically spares are available.

i. Deep bed filter-There is only one spare of this specific type of filter. Spare glass, gaskets, and O-rings are available for rebuilding. An exact spare deep bed filter is not available. Fabrication of a replacement would take 26 weeks, depending on the availability of sintered metal filters. A temporary substitute might be obtained in 1-2 weeks.

j. 2-line block at PS-431 position on BR-4-Polyethylene line connected from outlet of the deep bed filter to PVC ball valve on right side of 2-line block at PS-431 position on BR-4 in Cubicle 4.

k. FDV-440 position with standard FDV block (set top to left)—Previously described in Section 6.2.4.g above.

l. T-430-Refer to Section 6.2.4.h for discussion of T-430.

m. 2-line block at HCV-T430-1 on BR-4-Polyethylene line with PVC ball valve connected on left side of 2-line block that runs from Cubicle 4 through Cubicle 4/5 pass through into Cubicle 5 through Cubicle 5/6 pass through to Cubicle 6 into Cubicle 6 and connected to T2003 GN in Cubicle 6 right DC well; fabricated in house, and typically spares are available.

n. T2003 GN in Cubicle 6 right DC well-Standard Zr GN with polyethylene line connected to left side of 2-line block at HCV-T430-1 position; fabricated in house, and typically spares are available.

o. 2-line block at HCV-T430-1 on BR-4-Plug installed on right side to block T43005 line; fabricated in house, and typically spares are available.

p. 7PDV-P710-2 (Type B set to 2) on BR-7-To block P71004 line that tees into T2003 line; fabricated in house. Typically spares are available

q. CU4AJ-Cubicle 4 vacuum. Previously described in Section 4.2.3.j above.

r. Polyethylene tubing — For vacuum lines, pressure lines, demineralized water lines, and portions of the transfer route. Male ball joints and plastic compression fittings are readily available.

\subsubsection{HYDROXIDE Precipitation Filtrate Collection Tank, Tank T-20}

T-20 has several systems that are required to operate properly to maintain functionality. Typically, tank pit tanks like T-20 do not have many physical or mechanical issues that interrupt routine operations. Plugging instrument probes 
or broken instrument lines can pose some minor problems, but these problems are a low probability.

a. Tank T-20-A nominal $74 \mathrm{~L}$ tank constructed of HC with probes constructed of Zr. T-20 is located in Tank Pit 2 and is typically used to collect solutions from CLEANEX extractions and the HYDROXIDE precipitation operations.

b. Foxboro pneumatic instrument-T-20's liquid level and density are read out and recorded on an original Foxboro pneumatic instrument (over 40 years old). This instrument is typically reliable and is routinely calibrated. Broken polyethylene lines supplying the pneumatic signals to the transmitters and instrument can present some problems, but in recent years, UV-resistant polyethylene has decreased potential breaking of lines due to age and exposure to UV light. Plugged lines can be a problem if the plug cannot be removed easily.

c. T-20 sparger-An electric switch activates a solenoid that opens and allows air to flow into a deep leg in the tank for solution mixing in the tank. The air flow into the tank is regulated by an orifice plate in the sparge line. The orifice plate can plug requiring the suspension of sparging operations in T20. The orifice plate would have to be removed and cleaned. This typically only requires no more than $4 \mathrm{~h}$. Plugs in the sparge line downstream of the orifice or flow meter can occasionally occur, and this can sometimes require as many as $12-24 \mathrm{~h}$ to clear. The sparger solenoid has failed, even though failure is not frequent. Rebuilds and replacements are readily available and cause very minimal down time for repairs. If necessary an alternate method of sparging T-20 is by using the in-cell air and applying air pressure down the T-20 sample uptake line.

d. T-20 processing lines-These are the lines that are used to transfer process solutions into and out of T-20 and are constructed of Zr. Typically, these lines are not an issue, though at times some lines are preferred over others, and plugged lines can be a problem if the plug cannot be removed easily or an alternate line cannot be used.

e. T-20 sampler-A $1 \mathrm{~L}$ glass bottle with a 2-line rubber stopper for sampling in Cubicle 6 is connected to a T2002 GN using polyethylene tubing. Glass bottles, stoppers, polyethylene tubing with female ball joints, polyethylene tubing, and plastic compression fittings are kept in stock and typically readily available. (The designed sample technique is no longer employed because there are no needle blocks available to use the vacuum accumulators to pull samples from the tank pit tanks.)

6.2.10 Once the solution in T-61 has been filtered to T-20, the precipitate in T-61 is washed with $3 \times 4.5 \mathrm{~L}$ of $0.25 \mathrm{M} \mathrm{NaOH}$ and $2 \times 4.0 \mathrm{~L}$ of water added from M421 in the MUA to T-61. Refer to Section 6.2.6 for discussion of Tank M-421.

6.2.11 The precipitate in T-61 is dissolved by making acid additions to T-61 from M421. The routes and equipment have been previously discussed in Sections 6.2.6 
and 6.2.8 with the exception that the dissolved precipitate (product solution) is collected in T-43 instead of T-20 where the filtrate was collected. Refer to Sections 2.3.11 and 2.3.12 for discussion of Tank T-43.

6.2.12 T-61 is flushed to T-43 with $3 \times 2.5 \mathrm{~L}$ of $1 \mathrm{M} \mathrm{HNO}_{3}$ with $0.5 \mathrm{~L}$ water flush in and $3 \times 2 \mathrm{~L}$ of water added from M-421. Previously discussed in Section 6.2.6.

6.2.13 The dissolved precipitate product solution in T-43 is sparged for at least $8 \mathrm{~h}$ and sampled utilizing the method described in Section 2.3.11.e.

6.2.14 The filtrate in T-20 is also sparged and sampled utilizing the method described in Section 6.2.9.e above.

6.2.15 At the conclusion of the final batch of material to be processed, $3 \mathrm{~L}$ of $3 \mathrm{M} \mathrm{HNO}_{3}$ are added to T-61 for a heel check utilizing M-704 (can substitute an equivalent tank), sparged, and sampled. Refer to Section 1.4.2 for discussion of M-704. Refer to Section 3.4.7.e for T-61 sample method.

6.2.16 At the conclusion of the final batch of material to be processed, 2.5 $\mathrm{L}$ of concentrated $\mathrm{HNO}_{3}$ are added to T-60 from M-704 (can substitute an equivalent tank) and flushed in with enough water to bring the volume in T-60 to $20 \mathrm{~L}$ for a boiling heel check. T-60 is evaporated to $\sim 15 \mathrm{~L}$ volume, then sparged, and sampled. Refer to Section 1.4.2 for discussion of M-704. Refer to Section 6.2.1.e for T-60 sample method and Section 6.2.2 for evaporator information.

6.2.17 T-60, T-61, and T-20 solutions are dispositioned based on sample results.

\subsection{OXALATE Precipitation Operations}

7.1 Chemical Make Ups

7.1.1 All chemical make ups contain oxalic acid of varying concentration and are made up and stored in polyethylene containers. Typically, the oxalic acid is recorded during a routine inventory and ordered as needed. The containers used for the chemical make ups are readily available for use.

a. A solution of $0.8 M$ oxalic acid is used for precipitating the actinide product material. This is typically made up in a $10 \mathrm{~L}$ batch in a polyethylene container.

b. Wash solution ( $0.2 \mathrm{M}$ oxalic acid, $0.5 \mathrm{MHNO}_{3}$ ) is typically made up in a $14 \mathrm{~L}$ batch in a polyethylene container.

c. Oxalate rinse solution ( $0.2 \mathrm{M}$ oxalic acid) may or may not be used. If it is required, it is made up in a designated batch size in a polyethylene container.

\subsection{OXALATE Feed Adjustment}

The feed solution in T-54 is evaporated down to a prescribed volume or boiling temperature of $130^{\circ} \mathrm{C}$ whichever occurs first, then an initial acid adjustment is made by 
an addition of a prescribed amount and concentration of $\mathrm{HNO}_{3}$. T-54 is sparged and sampled, and a final acid adjustment of T-54 is made.

\subsubsection{T-54 OXALATE Feed Adjustment Tank, Evaporator T-54}

T-54 has several systems that are required to operate properly to maintain functionality. Typically, tank pit tanks like T-54 do not have many physical or mechanical issues that interrupt routine operations. Loss of steam and cooling water capabilities could have a negative impact, but this is a low probability.

a. T-54-A nominal 22 L HC shell with Ta liner evaporator tank located in Tank Pit 5. T-54 has an annulus filled with Hg for increased heat transfer capability and is used to evaporate the dissolved actinide product from the target rods as part of the hot feed adjustment for OXALATE precipitation operations.

b. Foxboro pneumatic instrument-T-54's liquid level and density are read out and recorded on an original Foxboro pneumatic instrument (over 40 years old). This instrument is typically reliable and is routinely calibrated. T-54 pressure is read out on an original Foxboro pneumatic instrument (also over 40 years old) that provides read outs for several tank pressures. Specific tank pressures provided on this instrument are selected for read out by a selector switch. Broken polyethylene lines supplying the pneumatic signals to the transmitters and instrument can present some problems, but in recent years, UV-resistant polyethylene has decreased potential breaking of lines due to age and exposure to UV light. Plugged lines can be a problem if the plug cannot be removed easily.

c. T-54 sparger-An electric switch activates a solenoid that opens and allows air to flow into a deep leg in the tank for solution mixing in the tank. The air flow into the tank is regulated by an orifice plate in the sparge line. The orifice plate can plug requiring the suspension of sparging operations in $\mathrm{T}$ 54. The orifice plate would have to be removed and cleaned. This typically only requires no more than $4 \mathrm{~h}$. Plugs in the sparge line downstream of the orifice or flow meter can occasionally occur, and this can sometimes require as many as $12-24 \mathrm{~h}$ to clear. The sparger solenoid has failed, even though failure is not frequent. Rebuilds and replacements are readily available and cause very minimal down time for repairs. If necessary an alternate method of sparging T-54 is by using the in-cell air and applying air pressure down the $\mathrm{T}-54$ sample uptake line.

d. T-54 processing lines-These are the lines that are used to transfer process solutions into and out of T-54. Typically, these lines are not an issue, though at times some lines are preferred over others, and plugged lines can be a problem if the plug cannot be removed easily or an alternate line cannot be used.

e. T-54 sampler-A $1 \mathrm{~L}$ glass bottle with a 2-line rubber stopper for sampling in Cubicle 4 is connected to a 1-line polyethylene block installed in the T-54 
needle block position (SA-T54) on BR-4 using polyethylene tubing. Glass bottles, stoppers, polyethylene tubing with female ball joints, polyethylene tubing, and plastic compression fittings are kept in stock and typically readily available. (The designed sample technique is no longer employed because there are no needle blocks available to use the vacuum accumulators to pull samples from the tank pit tanks.)

\subsubsection{T-54 Heating/Cooling}

a. Thermocouples-For monitoring the temperature during evaporation and feed adjustment. The thermocouples come off the tank in the tank pit and then run through the LAA wall and eventually go to the Control Room, where they read out on temperature instruments. The thermocouples are typically very reliable, but thermocouple failure necessitating thermocouple replacement is very difficult and time-consuming. Instrument failure could potentially cause significant delays as well. Thermocouple or temperature instrument failure would prevent use of T-54 as an evaporator.

b. T-54 steam jacket and associated steam and condensate lines and valvesUsed for heating the tank for evaporation. If the steam supply to the evaporator is shut down for any reason, T-54 cannot be evaporated. Manual valves that allow RCW or steam to flow through the heating/cooling jacket on T-54 are located in the MUA. There is also a solenoid valve on the condensate discharge from T-54 that is operated by an electrical switch in the Control Room. Typically, replacement parts are available for these valves, and most repairs are accomplished in less than $4 \mathrm{~h}$.

c. PIC-T54-The steam supply to T-54 is controlled at the main panel board in the Control Room by pressure indicator controller (PIC-T54), which supplies air pressure to a steam regulator, pressure control valve (PCV-T54), on the steam supply line to the T-54 steam jacket. Typically, the PICs are very reliable and rarely need repair or replacement. The PCVs can sometimes have diaphragm failures and leaks that require repair and occasional replacement. This can take up to $24 \mathrm{~h}$ depending on the extent of the repair.

d. T-54 RCW lines and valves-Used for cooling the tank. The RCW system supplies cooling water to T-54. Manual valves that allow RCW or steam to flow through the heating/cooling jacket on T-54 are located in the MUA. Typically, replacement parts are available for theses valves, and most repairs are accomplished in less than $4 \mathrm{~h}$.

e. Condenser on the VOG line-The H-54 condenser is located in the tank pit and has RCW supplied to it for condensing the vapor in the condensate line. The RCW supply to H-54 is controlled by manual valves located in the MUA. The operability of the condenser is very reliable. Spare parts are readily available for these valves, and most repairs are accomplished in less than $4 \mathrm{~h}$.

f. VOG-Used for ventilating the tank. During evaporation, vapor travels through condensers and the vapor is condensed and collected in a 
condensation collection tank. This line is intact, and no known reliability issues exist.

7.2.3 M-421 is used for making additions supporting the OXALATE precipitation operations. Refer to Section 6.2.6 for discussion of Tank M-421.

7.2.4 M-450 a nominal 2.4 L glass column (2 in. diameter $\times 48$ in. tall) tank. M-450 is located in the MUA and is used for solution additions to T-54, T-43, T-45, and the LR-4 tanks supporting the oxalate precipitation operations. Inlet lines into M450 come in through the top flange, and there is one outlet line exiting through the bottom flange. The inlet lines and outlet line have Jamesbury ball valves for air pressure, water and venting utilities, solution addition, and solution discharge. Typically, this type tank requires minimal maintenance, and spare parts are readily available if required. The ball valves sometimes develop leaks, but typically they are very reliable. Normally, leaking valves can be repaired, but replacement is usually about as cost-effective as repairing. The lines and valves associate with these tanks are listed below:

a. V-M450-1, vent line to VOG

b. V-M450-2, solution addition line through the funnel

c. V-M450-3, 5 psig air pressure supply line

d. V-M450-4, drain line/solution outlet

e. V-M450-7, demineralized water addition line

\subsubsection{P-460 Heating Loop}

a. P-460 pump-This pump is located in the MUA. It has been reliable, but its age is greater than 40 years old. Procuring a replacement has not been explored. It may be possible that a comparable pump could be scavenged from one of the other heating loops if necessary to avoid a significant time delay, but that would have to be determined to be cost-effective because most of the other possible replacement pumps are very old as well. The time, cost, and effort may not warrant using a possibly unreliable pump in this situation. It would be better to procure an equivalent replacement. Procuring and installing an acceptable replacement might require a significant amount of time (as much as 4 weeks or longer).

b. V-P460-1, V-P460-2, V-P460-5, V-P460-6, V-P460-7, V-CAL260-1, V-CAL260-2, V-CAL260-3, V-CAL260-4, V-CAL260-5, V-CAL260-6, VCAL260-7, and PRV-P460-3-Valves part of the P-460 heating loop system. All the valves appear to be functional as observed during their last use in mid-2013. All these valves, but one, are a combination of gate and ball valves from various manufacturers and equivalent replacements for these valves are kept on hand. Typically, these do not experience instantaneous complete failure. They usually start leaking, and the leak gets worse to the point that it is desirable to change the valve. The one valve that is not a ball or gate valve is a pressure relief valve. The valve change out would typically 
be a2-4 h job, depending on whether or not the loop was being used just prior to the change out and had to cool down before being worked on.

c. H-460 heater-This heater has not been replaced in over 40 years. It has been reliable, but its age could be factor. Procuring a replacement has not been explored. Another comparable heater may not be available to be scavenged from one of the other heating loops to avoid a significant time delay. The lead time for procuring and installing an acceptable replacement could be 2-4 weeks.

d. TC-H460 temperature controller for the H-460/P-460 heating loop-This controller has been in service for approximately 15 years with little or no problems experienced in all that time. This is current controller so far has proven very reliable. A spare is not available, but procurement of an acceptable replacement in 1-2 weeks is probable.

e. FI-P460-This flow indicator has proven to be reliable. It has required very little or no maintenance for over 40 years of service. It is unlikely that a total failure of the flow indicator would occur. Currently, there are no spares on hand, but acceptable replacements could be procured and installed relatively quickly.

f. H-460 thermocouple-This is an off the shelf Type K thermocouple. Failure of the thermocouple can be instantaneous with no warning or a gradual failure with some indication that the thermocouple is about to fail. This thermocouple is critical for performing the precipitation operations in T-451.

7.3 OXALATE Feed Transfer to Precipitator, First Cycle Precipitation, and Second Cycle Precipitation Operations

\subsubsection{Hot Feed Transfer}

The hot feed is transferred to the precipitator tank, T-451.

Cell equipment utilized:

a. T5407 GN in Cubicle 4 right DC well—Standard Zr GN with polyethylene line connected to special FDV at FDV-440; fabricated in house. Typically spares are available.

b. Special FDV at FDV-440 position on BR-4 (special polyethylene block)— Fabricated in house. Typically spares are available.

c. T-430 on BR-4-Refer to Section 6.2.4.h for discussion of Tank T-430.

d. HCV-T430-1 position (utilizing a PVC block toggle hand valve) on BR-4Fabricated in house. Typically spares are available (a special 2-line HCV block with a polyethylene line and Haywood PVC ball valve connecting the two lines might be substituted for the HCV toggle valve, and spare Haywood PVC valves are readily available).

e. F-432 position on BR-4-Consists of a 3 in. $\times 4.75$ in. glass pipe, flat gaskets, O-ring, and specific porosity $(10 \mu \mathrm{m})$ filter element. Typically, there are spare glasses, gaskets, O-rings, and filter elements available. These 
standard in-cell filter housings are typically reliable pieces of equipment that are fabricated in house. Spare housing can be swapped out and borrowed from other positions in the hot cell cubicles as necessary.

f. HCV-F432-2 position (utilizing a PVC block toggle hand valve) on BR-4Fabricated in house. Typically spares are available (a special 2-line HCV block with a polyethylene line and Haywood PVC ball valve connecting the two lines might be substituted for the HCV toggle valve, and spare Haywood PVC valves are readily available).

g. F43202 GN on BR-4-With polyethylene line to T45105 GN on LR-4.

h. 7PDV-T451-1 position (Type D set to 7) on BR-4-Fabricated in house. Typically spares are available.

i. HCV-T451-1 position (utilizing a PVC block toggle hand valve) on LR-4Fabricated in house. Typically spares are available (a special 2-line HCV block with a polyethylene line and Haywood PVC ball valve connecting the two lines might be substituted for the HCV toggle valve, and spare Haywood PVC valves are readily available).

j. T-451on LR-4-Nominal 5 L glass tank with an electrical stirrer for mixing. The top is $\sim 6$ in. ID, and it necks down to a smaller diameter ( $\sim 1 / 2$ in. ID) at the bottom, which is also flanged. T-451 has a spray ring located in the upper portion of the tank for distributing the oxalic acid for precipitation and also for flushing down the walls of the tank. The stirrer consists of an electrical motor mounted on the top of the top flange coupled to the stirrer extending into the tank vertically. The stirrer is controlled by a Cole Parmer Servodyne controller mounted outside the cubicle at the cubicle face. There is at least one spare glass available for T-451, but it has not been calibrated for use. This tank has proved to be somewhat fragile when being removed and reinstalled after repair work in the Decon Box. There have been issues with the stirrer over the years. The motor housing and bearing housing have a clean air purge that is supposed to protect the motor and bearings from corrosion caused by chemical exposure from the acids and bases added to T451 during oxalate processing, but effectiveness of the purge has been questioned because of the frequency of failure of the bearings. The motors have tended to be more reliable over the years. There are a few spare motors left at the REDC, but the manufacturer does not make this particular motor any more. A replacement motor is in process of being tested for another program that has utilized the old type T-451 stirrer motor. There are enough original model spares to last many years. It would take $\sim 1-1.5$ weeks to effect repairs on the T-451 stirrer considering that everything went well and it was an ordinary bearing failure or motor failure. It could take as much as 3 or more weeks if the failure was more catastrophic, such as damaging the shaft. There are some spare Servodyne controllers, but recently it has been difficult to keep these controllers in good working order. There are several working controllers that can be borrowed if a failure occurs. 
7.3.2 OXALATE filtrate collection tanks (T-456, T-457, and T-458) and knock out tank (T-459) located on LR-4 behind the valves, pumps, filters, and other equipment. These tanks, with the exception of T-459, collect the filtrate of the precipitated product, and each of the collection tanks has a nominal volume of 18.2 L. The Knock Out Tank, T-459, has a nominal volume of 10.0 L. Each tank is constructed of $\mathrm{Zr}$ and is equipped with pneumatic liquid level probes that read out on the Master Panel Board, MB-20, in the Control Room. Each tank has a sample line to a special needle block/modified syringe assembly for sampling, an inlet line from a common header on LR-4 for collection of solutions from the OXALATE processing operations, and two deep lines for solution removal. The probes have a slight probability of plugging during operations, which could create difficulty in the confidence of the actual volume in the tank, but typically other methods can be employed to ensure that any of the tanks will not be overfilled. There have been no significant problems with these tanks other than the aforementioned infrequent plugging of the liquid level probes.

\subsubsection{First Cycle Precipitation}

a. Once the hot feed has been transferred to T-451, the P-460 heating loop is set up to heat T- 451 to $60^{\circ} \mathrm{C}-65^{\circ} \mathrm{C}$. Refer to Section 7.3.1.j for discussion of T451, and refer to Section 7.2.5 for discussion of the P-460 heating loop.

b. The T-451 agitator is set to mix at 1,000-2,500 rpm. Refer to Section 7.3.1.j for discussion of the T-451 agitator.

c. $\quad 1.0 \mathrm{~L}$ of $0.8 \mathrm{M}$ oxalic acid is slowly added to T-451 at a rate of $200 \pm 50 \mathrm{ml} / \mathrm{min}$ from M-450 down the V-M450-5 valve in the MUA. Refer to Section 7.2.4 for discussion of M-450 and associated valves. This is followed by $400 \mathrm{ml}$ of $0.2 \mathrm{M}$ oxalic acid mixed with $0.5 \mathrm{M} \mathrm{HNO}_{3}$ (wash solution). Additional wash solution is added until the level in T-451is between 4.3 and $4.5 \mathrm{~L}$.

d. T-451 is mixed for 15 min while the tank is maintained at $60^{\circ} \mathrm{C}-65^{\circ} \mathrm{C}$.

e. The P-460 heating loop is set to cool, and then T-451 solution is pumped to T-456. Refer to Section 7.3.2 for discussion about T-456.

f. T-451 is flushed to T-456 with a minimum of $3 \times 0.5 \mathrm{~L}$ of wash solution ( $0.2 \mathrm{M}$ oxalic acid and $0.5 \mathrm{MHNO}_{3}$ ). An additional flush can be added if needed.

\subsubsection{First Cycle Precipitation Transfer Route}

The transfer route is from T-451 to T-456.

Cell equipment utilized:

a. T-451-Refer to Section 7.3.1.j for discussion of the T-451 and its features.

b. 1-line block at HV-T451-2 position (line at the left) on LR-4-With PVC ball valve and polyethylene line connected from line to a 1-line block at 7PDV-T451-2. Fabricated in house. Typically spares are available. (The discharge line on LR-4 from HV-T451-2 is leaking; therefore, an alternate 
route has to be used that requires a special 1-line block at the HV-T451-2 position with a polyethylene line connected to a special 1-line block at 7PDV-T451-2 on LR-4.)

c. Special 1-line 7PDV Type D polyethylene block at 7PDV-T451-2 position (set to 5) on LR-4 with polyethylene line from 1-line block at HV-T451-2Fabricated in house. Typically spares are available. (The discharge line on LR-4 from HV-T451-2 is leaking; therefore, an alternate route has to be used that requires a special 1-line block at the HV-T451-2 position with a polyethylene line connected to a special 1-line block at 7PDV-T451-2 on LR-4.)

d. F-454 on LR-4-Metal fritted filter mounted on LR-4 with manual stirrer operated by moving a lever arm located on top of the filter back and forth. The spare filter was installed in May 2014. No other spares are currently available.

e. FDV-454 position (set top to left) on LR-4—Fabricated in house. Typically spares are available.

f. HCV-P452-1 position (utilizing a PVC block toggle hand valve) on LR-4Fabricated in house. Typically spares are available.

g. P-452 vacuum/pressure diaphragm pump on LR-4 (referred to as a TRU pump because it was designed specifically for use in this facility)—No real spares available. Pumps can be rebuilt with significant effort. There are several nonfunctioning spare pumps available for rebuilding. Current Hot Cell Operations personnel have little or no experience in rebuilding these types of pumps. Spare parts to rebuild the pumps are limited at this writing.

h. FDV-P452 (set top to right) on LR-4—Fabricated in house. Typically spares are available.

i. HCV-T456 position (utilizing a PVC block toggle hand valve) on LR-4 (opened for first cycle transfer)—Fabricated in house. Typically spares are available.

j. HCV-T457 position (utilizing a PVC block toggle hand valve) on LR-4 (closed for first cycle transfer)-Fabricated in house. Typically spares are available.

k. HCV-T458 position (utilizing a PVC block toggle hand valve) on LR-4 (closed for first cycle transfer)—Fabricated in house. Typically spares are available.

1. T-456-Refer to Section 7.3.2 for discussion of T-456.

MUA equipment utilized:

m. M-450 and associated valves-Used for additions to T-451. Refer to Section 7.2.4 for discussion of M-450 and associated valves.

\subsubsection{Dissolution of the First Cycle Precipitate}

a. For the initial dissolution of a series, concentrated $\mathrm{HNO}_{3}$ is added to T-451 from M-450. Refer to Section 7.3.1.j for discussion of T-451, and refer to 
Section 7.2.4 for discussion of M-450 and associated valves. On the second and later dissolutions, acid for dissolution is transferred from T-458 to T-451 as discussed in Section 7.3.6 below.

b. The acid is allowed to set in T-451 for 10-15 min to dissolve any residual precipitate in T-451.

c. The acid is then pumped from T-451 through F-454 to T-458 using P-452 utilizing the route discussed in Section 7.3.4.a-k . The receiving tank is T458. Refer to Section 7.3.2 for discussion of T-458.

d. T-451 is flushed through F-454 to T-458 with $2 \times 300 \mathrm{ml}$ water utilizing the same route.

\subsubsection{Transfer and Flush of Product Solution to T-451}

a. On the second and later dissolutions, acid for dissolution is transferred from T-458 to T-451 by setting 7PDV-T451-1 (Type D set to 5) and using the cubicle vacuum connected to T-451 to draw the solution into T-451. Refer to Section 7.3.1.h for discussion of 7PDV-T451-1. Refer to Section 7.3.1.j and Section 7.3.2 for discussion of T-451 and T-458, respectively.

b. T-458 is flushed to T-451 with $2 \times 300 \mathrm{ml}$ water. Each flush is added from M-450. Refer to Section 7.2.4 for discussion of M-450 and associated valves.

\subsubsection{Second Cycle Precipitation}

a. The P-460 heating loop is set up to cool T-451. Refer to Section 7.2.5 for discussion of the P-460 heating loop.

b. Once the first cycle dissolved product has been transferred to T-451, the acid is adjusted by adding a prescribed amount of concentrated $\mathrm{NH}_{4} \mathrm{OH}(14 M)$ from M-450. Refer to Section 7.2.4 for discussion of M-450 and associated valves.

c. The T-451 agitator is set to mix at 1,000-2,500 rpm. Refer to Section 7.3.1.j for discussion of the T-451 agitator.

d. T-451 is sampled for acid concentration. T-451 has a sample line to a special needle block/modified syringe assembly for sampling.

e. T-451 acid concentration may be adjusted with appropriate additions of $\mathrm{HNO}_{3}$ or $\mathrm{NH}_{4} \mathrm{OH}$ if necessary from $\mathrm{M}-450$.

f. Once the acid concentration in T-451 is acceptable, the P-460 heating loop is set up to heat T-451 to $60^{\circ} \mathrm{C}-65^{\circ} \mathrm{C}$. Refer to Section 7.2.5 for discussion of the P-460 heating loop.

g. $\quad 1.0 \mathrm{~L}$ of $0.8 \mathrm{M}$ oxalic acid is slowly added to $\mathrm{T}-451$ at a rate of $200 \pm 50 \mathrm{ml} / \mathrm{min}$ from M-450 down the V-M450-5 valve in the MUA. This is followed by $400 \mathrm{ml}$ of $0.2 \mathrm{M}$ oxalic acid- $0.5 \mathrm{MHNO}_{3}$ (wash solution). Additional wash solution is added until the level in T-451 is between 4.3 and $4.5 \mathrm{~L}$. Refer to Section 7.2.4 for discussion of M-450 and associated valves.

h. T-451 is mixed for 15 min while the tank is maintained at $60^{\circ} \mathrm{C}-65^{\circ} \mathrm{C}$.

i. The P-460 heating loop is set to cool, then T-451 solution is pumped to T-458. Refer to Section 7.3.2 for discussion about T-458. 
j. $\quad$ T-451 is flushed to T-458 with a minimum of $3 \times 0.5 \mathrm{~L}$ of wash solution (0.2 $\mathrm{M}$ oxalic acid, $0.5 \mathrm{M} \mathrm{HNO}_{3}$ ). An additional flush can be added if needed.

7.3.8 Second Cycle Precipitation Transfer Route

a. The transfer route is from T-451 to T-458. The transfer route and associated equipment is discussed in Section 7.3.4.a-j.

b. T-458-Refer to Section 7.3.2 for discussion about T-458.

7.3.9 Dissolution of the Second Cycle Precipitate

a. Concentrated $\mathrm{HNO}_{3}$ is added to T-451 from M-450. Refer to Section 7.3.1.j for discussion of T-451. Refer to Section 7.2.4 for discussion of M-450 and associated valves.

b. The acid is allowed to set in T-451 for 10-15 min to dissolve any residual precipitate in $\mathrm{T}-451$.

c. The acid is then pumped from T-451 through F-454 to T-43 using P-452 utilizing the route discussed in Section 7.3.4.a-k. The receiving tank is T-43. Refer to Section 2.3.11 and Section 2.3.12 for discussion of T-43.

7.3.10 System and Equipment Flushing

The transfer systems and equipment are flushed prior to the next OXALATE run in a series.

\subsubsection{T-43 OXALATE Product Tank Sampling and Evaporation}

a. T-43 is sparged and sampled following each complete run in a series as prescribed in the OXALATE procedure. Refer to Section 2.3.11.c for discussion of T-43 sparger and Section 2.3.11.e for discussion of T-43 sampling method.

b. T-43 is evaporated as necessary to maintain adequate free board for the next batch of product. Refer to Sections 2.3.11 and 2.3.12 for discussion of T-43 evaporation.

c. After the final run of a series, T-43 may be sampled and evaporated in preparation for the feed adjustment for the Cm OXIDE operations as described in Section 7.3.11.a above.

\subsection{Curium OXIDE Operations}

\subsection{OXIDE Feed Adjustment}

NOTE: The feed solution for the OXIDE operations in T-43 may have had accountability samples pulled at the end of the OXALATE operations, but if accountability samples were not pulled, the OXIDE feed adjustment procedure will include pulling accountability samples from T-43. 
8.1.1 The feed solution in T-43 is evaporated at a normal evaporation rate until the boiling temperature reaches $119^{\circ} \mathrm{C}$, then the evaporation rate is adjusted so that $\mathrm{T}-43$ is maintained at $>119^{\circ} \mathrm{C}$ boiling temperature for $>5 \mathrm{~h}$ to destroy the oxalate from the previous OXALATE operations. Refer to Sections 2.3.11 and 2.3.12 for discussion of heating and cooling of T-43.

8.1.2 After the oxalate destruction is completed, T-43 is evaporated to dryness.

8.1.3 A prescribed amount of $0.1 \mathrm{MHNO}_{3}$ is added back to T-43 using M-421 and associated valves. Refer to Section 6.2.6 for discussion of M-421.

8.1.4 T-43 is heated up to between $80^{\circ} \mathrm{C}$ and $100^{\circ} \mathrm{C}$ and simmered for $30 \mathrm{~min}$, then cooled to $<50^{\circ} \mathrm{C}$. Refer to Sections 2.3.11 and 2.3.12 for discussion of heating and cooling of T-43.

8.1.5 T-43 is sampled to confirm that the acid concentration is in the proper range. If it is not, it is adjusted by an acid addition or repeating the boil down to dryness and rediluting with $0.1 \mathrm{MHNO}_{3}$. Refer to Section 2.3.11.e for discussion of T-43 sampler.

8.1.6 Once the final acid concentration is in the desired range, T-43 is evaporated down to give a final acid concentration of $0.2-0.35 \mathrm{~N}$, and a final actinide concentration of $\sim 10 \mathrm{~g} / \mathrm{L}$.

\subsection{System Set Up and Check Out}

8.2.1 A specially fabricated quartz column with a quartz frit in the bottom is used to hold resin for loading the hot feed and is installed on LR-4 at the C-448 position. Historically, these columns have been fabricated by the ORNL glass shop per engineering drawings or sketches originated from engineering drawings. Typically, an adequate supply of quartz columns is on hand before beginning the OXIDE operations. If excessive breakage of columns (because quartz is somewhat brittle by nature) or other unexpected problems reduce the inventory of spare columns, it may take several weeks to procure the necessary quartz components and have the glass shop fabricate replacements. If there is already a quartz column available in Cubicle 4 for use, then the quartz column is leached prior to use for a series of OXIDE runs.

8.2.2 A calibrated $1 \mathrm{~L}$ polyethylene bottle is filled with $0.25 \mathrm{MHNO}_{3}$ for use in a $250 \mathrm{ml}$ squeeze bottle to leach the quartz column in Cubicle 4, if the quartz column is being reused.

8.2.3 Leach of the C-448 quartz column with $0.25 \mathrm{M} \mathrm{HNO}_{3}$ (this is done after the first run of a series). The column is filled with $0.25 \mathrm{M} \mathrm{HNO}_{3}$ and the solution is pumped to T-43 and then flushed to T-43 with water.

Cell equipment utilized: 
a. C-448-Quartz column. Refer to Section 8.2.1 for discussion of the quartz column.

b. P44601 GN position on LR-4-A special male polyethylene ball joint and JACO elbow installed on this standard Zr GN (this is connected to the bottom of the C-448 quartz column and in effect supports the column when installed on LR-4). Spare special male ball joints and elbow made in house and are readily available. Spare GNs are typical available as well.

c. HCV-P446-1 position on LR-4-A special pass through polyethylene block is installed; fabricated in house. Typically spares are available.

d. P-446 vacuum/pressure diaphragm pump on LR-4—Referred to as a TRU pump because it was designed specifically for use in this facility. No real spares available. Pumps can be rebuilt with significant effort. There are several nonfunctioning spare pumps available for rebuilding. Current Hot Cell Operations personnel have little or no experience in rebuilding these types of pumps. Spare parts to rebuild the pumps are limited at this writing.

e. RE-P446 position on LR-4-Pass through installed in the position; fabricated in house. Typically spares are available

f. HCV-P446-2 position (utilizing a PVC block toggle hand valve) on LR-4Fabricated in house. Typically spares are available.

g. T-43-Refer to Sections 2.3.11 and 2.3.12 for discussion of T-43.

8.2.4 DOWEX 50-X8 $(60-80 \mu \mathrm{m})$ resin-Resin is measured out in prescribed batch sizes and loaded into the quartz column. Resin is typically obtained and classified well ahead of the time that it is required for the OXIDE operations.

8.2.5 T-45 and T-43 - The loading raffinates from the OXIDE runs go to T-45 and T43. Refer Section 8.2.6 for discussion of T-45 and to Sections 2.3.11 and 2.3.12 for discussion of T-43.

\subsubsection{OXIDE Operations Loading Raffinate Collection Tank, Tank T-45}

T-45 has several systems that are required to operate properly to maintain functionality. Typically, tank pit tanks like T-45 do not have many physical or mechanical issues that interrupt routine operations. Plugging instrument probes or broken instrument lines can pose some minor problems, but these problems are a low probability.

a. Tank T-45-A nominal 24.7 L tank constructed of Zr with probes constructed of Zr. T-45 is located in Tank Pit 4 and is typically used to resin loading raffinates from the OXIDE operations.

b. Foxboro pneumatic instrument-T-45's liquid level and density are read out and recorded on an original Foxboro pneumatic instrument (over 40 years old). This instrument is typically reliable and is routinely calibrated. Broken polyethylene lines supplying the pneumatic signals to the transmitters and instrument can present some problems, but in recent years, UV-resistant polyethylene has decreased potential breaking of lines due to age and 
exposure to UV light. Plugged lines can be a problem if the plug cannot be removed easily.

c. T-45 sparger-An electric switch activates a solenoid that opens and allows air to flow into a deep leg in the tank for solution mixing in the tank. The air flow into the tank is regulated by an orifice plate in the sparge line. The orifice plate can plug requiring the suspension of sparging operations in T45. The orifice plate would have to be removed and cleaned. This typically only requires no more than $4 \mathrm{~h}$. Plugs in the sparge line downstream of the orifice or flow meter can occasionally occur, and this can sometimes require as many as $12-24 \mathrm{~h}$ to clear. The sparger solenoid has failed, even though failure is not frequent. Rebuilds and replacements are readily available and cause very minimal down time for repairs. If necessary an alternate method of sparging T-45 is by using the in-cell air and applying air pressure down the T-45 sample uptake line.

d. T-45 processing lines-These are the lines that are used to transfer process solutions into and out of T-45. Typically, these lines are not an issue, though at times some lines are preferred over others, and plugged lines can be a problem if the plug cannot be removed easily or an alternate line cannot be used.

e. T-45 Sampler-A $1 \mathrm{~L}$ glass bottle with a 2-line rubber stopper for sampling in Cubicle 4 is connected to a 1-line polyethylene block installed in the T-45 needle block position (SA-T45) on BR-4 using polyethylene tubing. Glass bottles, stoppers, polyethylene tubing with female ball joints, polyethylene tubing, and plastic compression fittings are kept in stock and typically readily available. (The designed sample technique is no longer employed because there are no needle blocks available to use the vacuum accumulators to pull samples from the tank pit tanks.)

\subsection{Feed Transfer and Resin Loading}

8.3.1 Once the system set up and check out and feed adjustment are complete, the feed is transferred from T-43 in prescribed batch sizes for each run to T-444 per the route below.

Cell equipment utilized:

a. FDV-435 (set top to right) on BR-4-Fabricated in house. Typically spares are available.

b. FDV-T444-1 (set top to right) on LR-4-Fabricated in house. Typically spares are available.

c. F-445 position on LR-4 with special pass through polyethylene block or special 2-line block with polyethylene tubing jumper-Fabricated in house. Typically spares are available.

d. T-444-Refer to Section 6.2.8.g for discussion of T-444. 
8.3.2 The hot feed is then pumped from T-444 through the C-448 resin column using P446. The raffinate is collected in T-45 until breakthrough is observed in C-448, and then the discharge is rerouted to T-43. The route is outlined below:

a. HCV-T444-2 position (utilizing a PVC block toggle hand valve) on LR-4Fabricated in house. Typically spares are available.

b. FDV-T444-3 (set left to right) on LR-4Fabricated in house. Typically spares are available.

c. C-448-Quartz column. Refer to Section 8.2.1 for discussion of the quartz column.

d. HCV-P446-1 position (utilizing a PVC block toggle hand valve) on LR-4Fabricated in house. Typically spares are available.

e. P-446 vacuum/pressure diaphragm pump on LR-4-Previously discussed in Section 8.2.3.d..

f. $\quad$ RE-P446 position on LR-4-Previously discussed in Section 8.2.3.e.

g. HCV-P446-2 position (utilizing a PVC block toggle hand valve) on LR-4Previously discussed in Section 8.2.3.f.

h. T-43-Valve set to collect C-448 discharge in T-43. Refer to Sections 2.3.11 and 2.3.12 for $\mathrm{T}-43$ evaporator discussion.

i. $\quad$ T-45-Valve set to collect C-448 discharge in T-45. Refer to Section 8.2.6 for discussion of $\mathrm{T}-45$.

j. M-421-Used for route flushing with demineralized water supporting the Curium OXIDE operations. Refer to Section 6.2.6 for discussion of Tank M421.

k. FDV-T444-2 position (set top to right) on LR-4—Fabricated in house. Typically spares are available.

l. HCV-T444-1 position (utilizing a PVC block toggle hand valve) on LR-4Fabricated in house. Typically spares are available.

m. CU4AJ_Cubicle 4 vacuum. Previously described in Section 4.2.3.j above.

n. Polyethylene tubing-For vacuum lines, pressure lines, demineralized water lines, and portions of the transfer route. Male ball joints and plastic compression fittings are readily available.

8.3.3 If the resin has not completely loaded and this is not the last run of the series, then Sections 8.3.1 and 8.3.2 above may be repeated to transfer an additional $250 \mathrm{ml}$ of solution from T-43 into T-444 and pump through C-448 to T-45/T-43.

8.3.4 Once C-448 is fully loaded (unless it is the last run of the series) and T-444 is pumped empty, T-444 is flushed with $2 \times 200 \mathrm{ml}$ of water. Each flush is pumped until the system is empty. Refer to Section 8.3.2 for discussion of the transfer route for the flushes.

NOTE: The in-cell demineralized water is throttled back to minimize the flow to ensure that no resin is splattered out during the flushing described below in Section 8.3.5. 
8.3.5 Once T-444 is pumped empty after the flushes, the stopper is carefully removed from C-448, and any resin is flushed off into C-448 using the in-cell demineralized water, then $200 \mathrm{ml}$ of water is added directly to the column and pumped to T-43.

8.3.6 The pump is allowed to operate an additional $1 \mathrm{~h}$ until the resin in C-448 is dry, and then P-446 is turned off. Refer to Section 8.3.2.e for discussion of P-446.

8.3.7 T-45 is acidified, sparged, and sampled for disposition. Refer to Section 8.2.6.e for discussion of T-45 sampler.

8.3.8 If this was not the last run of the series, the volume of the hot feed solution in T43 is adjusted to the prescribed volume by evaporation prior to starting the next run of the series. Refer to Sections 2.3.11 and 2.3.12 for T-43 evaporator discussion.

8.4 Resin Burning and Calcination of Actinide Oxide

8.4.1 C-448 Quartz Column Firing in the Cubicle 4 Round Furnace, H-419

a. H-419 round furnace located on LR-4-The quartz column (C-448) is removed from its position on LR-4 and placed into the $\mathrm{H}-419$ round furnace such that the bottom of the quartz column that is protruding out of the bottom of the round furnace. The round furnace is fabricated in house. Currently, there is not a working spare available. There are some spare parts available for fabricating a spare round furnace, but it would require several weeks to complete the fabrication since all the necessary components have not been procured. Additional spare heating elements are not readily available.

b. Quartz top-The quartz top (outfitted with a frit in the top) is placed on top of the column. Spare quartz tops are typically readily available.Quartz lidThe lid is placed on to the round furnace.

c. Polyethylene tubing-An approximate $9 \mathrm{ft}$ length of $1 / 4$ in. polyethylene tubing with a male ball joint on one end and the other end connected to the purge line GN from the MUA (Cubicle 4 L-7 service position). These $1 / 4 \mathrm{in}$. polyethylene lines are typically made up ahead of time so adequate spares are available.

d. TIC-419 furnace controller-The temperature controller for H-419 Round Furnace is a Thermolyne Model 808. This particular model is no longer available, but comparable models from Eurotherm are available. No exact replacement spares are on hand. Other manufacturers of temperature controllers also have comparable controllers that could be substituted for the current model. Lead time for procurement and installation is about12 weeks.

e. Gas cylinders - The column is purged first with air, then $\mathrm{Ar}-\mathrm{H}_{2}$ gas, and finally again with air. Typically, supplies of $\mathrm{Ar}-\mathrm{H}_{2}$ gas cylinders are available as needed. 
f. H-419-Once the prescribed firing sequence is completed, the furnace is turned off, and the furnace and column are allowed to cool. Polyethylene tubing - The purge line is disconnected from the bottom of the quartz column, and the column removed from the round furnace.

\subsubsection{Calcination of Actinide Product in the Muffle Furnace, H-520}

a. Platinum crucible-The burned resin is carefully poured from the quartz column to a Pt lined Inconel crucible (Pt crucible). Currently there are no spare Pt crucibles, but efforts are underway to procure at least one or more spares.

b. Quartz column - The quartz column is reinstalled in position on LR-4 and then leaching of the column is performed per Section 8.2.3.

c. H-520 muffle furnace-The Pt crucible is placed in a special holder for firing in the muffle furnace (H-520). Previously described in Section 5.3.3 above.

d. Polyethylene lines-An air purge is set up for the muffle furnace prior to placing the Pt crucible inside the furnace for firing. A length of $1 / 4 \mathrm{in}$. polyethylene tubing with a male ball joint on one end and the other end connected to the purge line GN from the MUA Cubicle 4 R7 position. These $1 / 4$ in. polyethylene lines are typically made up ahead of time so adequate spares are available.

e. Box furnace-Once the preparations have been completed, the Pt crucible in its furnace holder is placed into the box furnace.

f. TIC-520 temperature controller-The furnace firing sequence is started using the furnace controller, TIC-H520, and the furnace is heated to a prescribed temperature and held for $16 \mathrm{~h}$ at this temperature. Previously described in Section 5.3.4 above.

g. Once the firing cycle of the actinide product is complete, the furnace is allowed to cool to $<250^{\circ} \mathrm{C}$. The furnace is opened, and the air purge disconnected.

h. The furnace is allowed to cool to $<100^{\circ} \mathrm{C}$, and then the Pt crucible with the calcined product is removed from the box furnace and held for characterization, sampling, and storage per Section 8.5 below.

8.4.3 If this was not the last run of the series, Section 8.2.4, Sections 8.3.1-8.3.8, and

Sections 8.4.1-8.4.2 are repeated until all the hot feed solution in T-43 is loaded and fired into oxide.

8.5 Characterization, Sampling and Storage of an Actinide Oxide Product

Each batch of oxide is characterized, sampled, and placed into a storage container.

8.5.1 The following equipment is required for characterization, sampling, and storage of the actinide oxide product. 
a. Stainless steel tray-Typically trays are available in the hot cell bank or if a new tray is required, new trays are available to be put into the hot cell cubicles as needed.

b. Certified/calibrated balance-Balances are typically available.

Certification/calibration of a new balance can be done ahead of time, but if a balance fails during use, it may require a couple of days to get a new balance certified/calibrated for use.

c. Certified standard weights_-Certified weight standards are available if needed.

d. Stainless steel screen assembly (bottom pan, $210 \mu \mathrm{m}$ screen with weight, and top) - There may not be a spare weight for the screen assembly, but the screen, bottom pan, and top are typically readily available with a less than 1 week lead time for procurement. The weight is a highly polished piece of stainless steel that may require at least a week to complete fabrication.

e. Aluminum weighing dish—_ Typically available as required.

f. Small glass powder funnel-Typically available as required.

g. Glass graduated cylinder (10 ml) - Typically available as required.

h. $250 \mathrm{ml}$ bottle holder-Used with $10 \mathrm{ml}$ graduated cylinder for tap density. Typically use one of the many that are already in the hot cell cubicles.

i. Stainless steel storage container-Typically fabricated as needed or use one of an existing storage container previously used to store oxide.

j. Glass sample vial-Typically available as required.

8.5.2 All the oxide characterization is performed over a stainless steel tray to catch any oxide that may spill Previously discussed in Section 8.5.1.a above.

8.5.3 The oxide is transferred from the Pt crucible to a screening assembly where it is screened to produce a specific consistent particle size Previously discussed in Sections 8.4.2.a and 8.5.1.d above.

8.5.4 A small sample is removed from the screen bottom pan and placed into a sample vial. The weight of the sample is obtained, and the sample is submitted to the analytical lab for analysis Previously discussed in Sections 8.5.1.a-j above.

8.5.5 The oxide is then transferred to a $10 \mathrm{ml}$ graduated cylinder for determining the tap density of the oxide. Previously discussed in Section 8.5.1.g above.

8.5.6 The oxide is finally transferred to a stainless steel storage container, and the final weight of the oxide is obtained. Previously discussed in Sections 8.5.1.b, 8.5.1.c, and 8.5.1.i above.

\subsection{Miscellaneous Process Support Systems and Equipment}

\subsection{VOG Scrubber, L-131, Operations}

The L-131 scrubber's primary function is to remove acid vapors from the VOG system prior to the VOG entering the high efficiency particulate air filters to protect the filters 
from corrosive acid vapors. The VOG from all the tank pit tanks, hot cell cubicles, and MUA tanks goes through the L-131 scrubber. The gas phase is contacted with the liquid solution in the scrubber as it flows up through a layer of packing in the upper portion of the scrubber. The liquid is pumped from the scrubber bottom to the top to flow down through the packing.

\subsubsection{VOG Scrubber Tank, L-131}

L-131 has many systems and ancillary equipment that are required for the scrubber to operate properly and to maintain functionality. Because the L-131 scrubber is more complex than the typical tank pit tanks, it can experience more physical or mechanical issues that interrupt routine operations from time to time.

a. L-131-A nominal $192 \mathrm{~L}$ tank that consists of a HC bottom section and a stainless steel top section with an internal HC support plate that hold stainless steel pall rings. The instrument lines and process lines are stainless steel. L-131 is located in Tank Pit 7 and is used to scrub the VOG.

b. Foxboro pneumatic instrument-L-131's liquid level and density are read out and recorded on a Foxboro pneumatic instrument (over 40 years old). This Foxboro instrument also includes a liquid level control capability used to maintain the liquid level at a desired level by regulating addition of demineralized water to the tank. This instrument is typically reliable and is routinely calibrated. Broken polyethylene lines supplying the pneumatic signals to the transmitters and instrument can present some problems, but in recent years, UV-resistant polyethylene has decreased potential breaking of lines due to age and exposure to UV light. Plugged lines are more prevalent in the L-131 scrubber that the usual tank pit tanks because of the chemical composition of the scrubber solution. Plugged lines can be a problem if the plug cannot be removed easily.

c. Yokogawa pneumatic instrument-L-131 pressure and differential pressure across the packing are read out and recorded on a newer Yokogawa pneumatic instrument ( 10 years old) with a digital readout and pen recorder. This instrument is routinely calibrated and is reliable. Broken polyethylene lines supplying the pneumatic signals to the transmitters and instrument can present some problems, but in recent years, UV-resistant polyethylene has decreased potential breaking of lines due to age and exposure to UV light. Plugged lines are more prevalent in the L-131 scrubber that the usual tank pit tanks because of the chemical composition of the scrubber solution. Plugged lines can be a problem if the plug cannot be removed easily.

d. L-131 probe flush down-Because L-131 is a critical component supporting the chemical processing operations, plugged instrument probes can present a serious problem; consequently, the instrument lines are equipped with quick disconnects so that water can be "plugged in" at the quick disconnect and used to pressurize and dislodge the plug. 
e. L-131 recirculation pumps, J-131A and J-131B-One pump running and one as a backup to pump the solution from the bottom of L-131 to above the packing in L-131 for contacting the vapors in the VOG flowing through the scrubber. The pumps are located in the pipe tunnel beneath the LAA floor blocks. These pumps have been changed out numerous times over the last 47 years. Typically, the pumps last between 10 and 15 years. It is uncertain that there are any spare pumps available and whether an exact replacement can be procured. If one pump fails, then there is a backup, but in the event that both pumps fail, processing would be suspended until suitable replacements were approved, procured, and installed. This could take as much as 1-2 months.

f. L-131 processing lines-These are the lines that are used to transfer solution into and out of L-131. Even though L-131 is not really considered a process tank because solution additions into and out of L-131 routinely occur. Typically, these lines are not an issue, but plugged lines can be a problem if the plug cannot be removed easily or an alternate line cannot be used. In the case of L-131, the number of available alternate lines for addition and removal of solutions is limited.

g. L-131 liquid level controller-LC-L131 is located on MB-1 as part of the liquid level and density recorder (LRC/DR-L131) for L-131. LCV-L131 valve controls the liquid level at the prescribed set point on LC-L131. The controller pneumatically operates a control valve, LCV-L131-2, that opens and closes based on whether or not demineralized water needs to be added to L-131 to maintain the prescribed level. It is necessary to maintain the L-131 level with water additions due to evaporation of the scrubber solution from recirculation and contact with the high volumetric flow of the VOG.

h. L-131 sampler-A 1 L glass bottle with a 2-line rubber stopper for sampling in Cubicle 7 is connected to a 1-line polyethylene block installed in the L131 needle block position (SA-L131) on BR-7 using polyethylene tubing. Glass bottles, stoppers, polyethylene tubing with female ball joints, polyethylene tubing, and plastic compression fittings are kept in stock and typically readily available. (The designed sample technique is no longer employed because there are no needle blocks available to use the vacuum accumulators to pull samples from the tank pit tanks.)

9.1.2 M-5 is a pressure transfer tank in the MUA used for the make up of solution for the L-131 scrubber. M-5 is a stainless steel tank that has been in use for over 40 years. M-5 is a nominal $140 \mathrm{~L}$ volume tank. M-5 is used mostly for transferring low concentration $\mathrm{NaOH}$ solutions, but during routine scrubber cleanouts, dilute $\mathrm{HNO}_{3}$ and demineralized water are transferred to L-131. The valves associated with M-5 are a mixture of Jamesbury, Apollo, Worchester, and Ernst stainless steel ball valves and a Hayward PVC ball valve. The ball valves sometimes develop leaks, but typically they are very reliable. Normally, leaking 
valves can be repaired, but replacement is usually about as cost-effective as repairing. The lines and valves associated with M-5 are listed below:

a. V-M5-1, vent line to VOG

b. V-M5-2, solution addition line through the funnel

c. V-M5-3, 10 psig air pressure supply line

d. V-M5-4, outlet line/drain from the bottom of the tank

e. V-M5-7, sparger for M-5 (10 psig supply air)

f. V-M5-8, outlet to L-131 (through LCV-L131)

g. V-M5-9, 10 psig air supply to sparger (tied to V-M5-1 vent)

h. V-M5-13, outlet/sample line

9.1.3 M-500 is a vacuum/pressure transfer tank used mainly for transferring caustic solutions from drums containing aqueous chemicals located in Room 212 adjacent to the MUA. M-500 is a nominal 15.2 L volume glass column (4 in. diameter $\times 72$ in. tall). Typically, the glass column tanks require very little maintenance. Replacement of the flange gaskets on glass column tanks is required very infrequently. There is also a filter in a stainless steel housing, F-500, installed on the suction line from the drum room. The valves associated with M-500 are a mixture of Jamesbury, Apollo, Worchester, and Watts stainless steel ball valves and one stainless steel gate valve. The valves sometimes develop leaks, but typically they are very reliable. Normally, leaking valves can be repaired, but replacement is usually about as cost-effective as repairing. The lines and valves associated with the tank used for the caustic solution make up are listed below:

a. V-M500-1, vent line to VOG

b. V-M500-2, vacuum supply

c. V-M500-3, 5 psig air pressure supply line

d. V-M500-4, outlet line/drain from the bottom of the tank

e. V-M500-5, outlet to M-40 (V-M40-5) and M-5 (V-M500-7)

f. V-M500-6, outlet

g. V-M500-7, inlet to M-5 from V-M500-5

h. V-M500-8, auxiliary inlet into M-500

i. V-F500-1, inlet from drum room caustic header into F-500

j. V-F500-2, inlet into M-500 from caustic header (bypass of F-500)

k. V-F500-3, drain valve for F-500

l. V-F500-5, inlet line from potassium hydroxide (KOH) $\mathrm{KOH}$ line into caustic header to F-500

m. V-F500-6, inlet line from $\mathrm{NaOH}$ line into caustic header to F-500

n. V-F500-7, inlet into $\mathrm{M}-500$ from $\mathrm{NaOH}$ line (bypass of $\mathrm{F}-500$ )

o. V-F500-8, outlet from F-500 to M-500

9.1.4 Routine L-131 Scrubber Liquid Change Out 
a. Routinely (bi-weekly), the solution in $\mathrm{L}-131$ is sampled for $\mathrm{OH}^{-}, \mathrm{CO}_{3}{ }^{-}$, and activities (gross alpha, alpha pulse, and gross neutrons). Refer to Section 9.1.1.h for discussion of L-131 sampling.

b. Routinely (bi-weekly) $\sim 25 \mathrm{~L}$ of the solution in the scrubber is drained to waste tank F-126 by operating HIC-J131 that opens HCV-J131A valve in the pipe tunnel. The HCV-J131A valve has been replaced several times. It is uncertain whether a spare valve for HCV-J131A is available or whether an exact replacement can be procured. The last time the valve was replaced was around 2003.

c. Replacement scrubber solution is made up in $\mathrm{M}-5$ by transferring $\mathrm{NaOH}$ from drums in Room 212 (drum room) to M-5 using the M-500 vacuum/pressure transfer tank. Refer to Sections 9.1.2-9.1.3 for discussion of M-5 and M-500.

d. After the solution is drained to F-126, the solution is replaced with $12.5 \mathrm{~L}$ of $\sim 5.0 \mathrm{M} \mathrm{NaOH}$ plus $2 \times 5 \mathrm{~L}$ water flushes. The solution is added from M-5 down the solution addition line to L-131.

\subsection{F-115 Waste Tank Operations}

\subsubsection{Aqueous Waste Collection, Tank F-115}

F-115 is located in the waste tank pit along with F-111 and F-126. F-115 has several systems that are required to operate properly to maintain functionality. Typically F-115 does not have many physical or mechanical issues that interrupt routine operations. Plugging instrument probes or broken instrument lines can pose some minor problems, but these problems are a low probability.

a. Tank F-115-A nominal 1,892 L tank constructed of HC with probes constructed of Zr. F-115 is located in the waste tank pit.

b. Yokogawa pneumatic instrument-F-115 liquid level, density, and pressure are read out and recorded on an Yokogawa recorder with a digital readout. This instrument is typically reliable and is routinely calibrated. Broken polyethylene lines supplying the pneumatic signals to the transmitters and instrument can present some problems, but in recent years, UV-resistant polyethylene has decreased potential breaking of lines due to age and exposure to UV light. Plugged lines can be a problem if the plug cannot be removed easily.

c. F-115 sparger-An electric switch activates a solenoid that opens a valve, HCV-F115-6, and allows air to flow into a deep leg in the tank for solution mixing in the tank. The air flow into the tank is regulated by an orifice plate in the sparge line. The orifice plate can plug requiring the suspension of sparging operations in F-115. The orifice plate would have to be removed and cleaned. This typically only requires no more than $4 \mathrm{~h}$. Plugs in the sparge line downstream of the orifice or flow meter can occasionally occur, and this can sometimes require as many as $12-24 \mathrm{~h}$ to clear. The sparger 
solenoid has failed, even though failure is not frequent. Rebuilds and replacements are readily available and cause very minimal down time for repairs. If necessary an alternate method of sparging F-115 is by using the incell air and applying air pressure down the F-115 sample uptake line.

d. F-115 processing lines-These are the lines that are used to transfer process solutions into and out of F-115. Typically, these lines are not an issue, though at times some lines are preferred over others, and plugged lines can be a problem if the plug cannot be removed easily or an alternate line cannot be used.

e. F-115 Sampler-A $1 \mathrm{~L}$ glass bottle with a 2-line rubber stopper for sampling in Cubicle 7 is connected to a 1-line polyethylene block installed in the F-115 needle block position (SA-F115) on BR-7 using polyethylene tubing. Glass bottles, stoppers, polyethylene tubing with female ball joints, polyethylene tubing, and plastic compression fittings are kept in stock and typically readily available. (The designed sample technique is no longer employed because there are no needle blocks available to use the vacuum accumulators to pull samples from the tank pit tanks.)

f. Steam waste jet-Typically, F-115 receives waste from the various process operations. Most of the process tanks in the tanks pits are piped with a direct route to F-115 via a steam waste jet. Those tanks that are not connected to F-115 via a steam waste jet can be routed to F-115 via a vacuum/pressure transfer tank in one of the four chemical processing hot cell cubicles.

9.2.2 F-115 waste solution disposition without a CLEANEX extraction stage present is handled following the steps below.

a. Once F-115 is $\sim 85 \%$ of its maximum volume, the solution in the tank is sparged and sampled for activities.

b. If activities meet prescribed limits, then the solution is transferred to waste tank F-126 via steam waste jet, J-F115. Refer to Section 9.3 for discussion of F-126.

c. If activities exceed prescribed limits, then a CLEANEX extraction stage is added to F-115 to recover the radionuclides from the solution. A CLEANEX extraction operation is then performed on the solution in F-115. This operation is discussed in Section 9.2.3 below.

9.2.3 F-115 waste solution disposition with a CLEANEX extraction stage present is handled following the steps below.

a. Once F-115 is $~ 85 \%$ of its maximum volume, the aqueous solution in the tank is sparged and sampled for disposal of the aqueous waste solution as prescribed in the CLEANEX extraction procedure.

b. The organic stage in F-115 will undergo many CLEANEX extractions once the organic stage is set up in the tank to recover material in the aqueous waste solution. The amount of material extracted into the organic, the amount 
of time the organic stage has been in F-115, and the schedule for upcoming process operation in the hot cells determines when the organic will be removed from F-115 and the material extracted into the organic recovered.

c. Once the material has been extracted in the F-115 organic stage, the aqueous raffinate is transferred to waste tank F-126 via steam jet. Refer to Section 9.3 for a discussion of F-126.

9.2.4 F-115 recovery CLEANEX operations are performed to recover the material that has been extracted into the organic stage in F-115 during the course of several months of routine chemical processing. Once the aqueous raffinate has been transferred to F-126, the organic in F-115 is removed (transferred) and flushed with water and diluent to T-77 for organic stripping operations per the following route:

Cell equipment utilized:

a. 1-line block at SA-F115 position on BR-7-Fabricated in house. Typically spares are available.

b. T73402 GN on RR-7—Standard Ta GN (Previously discussed in Section

1.6.1.d.) with polyethylene line connected to1-line block at SA-F115 position.

c. PS-733 position on RR-7-Refer to Section 2.3.2.c for discussion of PS-733.

d. HV-T734-2 on LR-7-Refer to Section 1.6.1.f for discussion of HV-T734-2.

e. T-734 on RR-7-Refer to Section 1.6.1.g for discussion of T-734.

f. HV-T734-3 on RR-7-Refer to Section 1.6.1.h for discussion of HV-T7343.

g. HV-T734-1 on RR-7-Refer to Section 1.6.1.k for discussion of HV-T7341.

h. T73404 GN on RR-7-Standard Ta GN outlet from T-734 (Previously discussed in Section 1.6.1.i.) with polyethylene line connected to T7703 GN located in Cubicle 7 right disconnect well.

i. $\quad$ T7703 GN in Cubicle 7 right DC well-Standard Zr GN inlet into T-77 with polyethylene line connected T73404 GN position on RR-7

j. T-77-Refer to Section 9.2.5 for discussion of T-77.

k. KP-735 on RR-7-Refer to Section 1.6.1.l for discussion of KP-735.

l. CU7AJ-In-cell air jet used for vacuum source. Previously discussed in Section 1.4.1.p.

m. Polyethylene tubing-For vacuum lines, pressure lines, demineralized water lines, and portions of the transfer route. Male ball joints and plastic compression fittings are readily available. Previously discussed in Section

\subsection{1.q}

\subsubsection{F-115 CLEANEX Stripping Tank, T-77}

T-77 has several systems that are required to operate properly to maintain functionality. Typically, tank pit tanks like T-77 do not have many physical or 
mechanical issues that interrupt routine operations. Plugging instrument probes or broken instrument lines can pose some minor problems, but these problems are a low probability.

a. T-77-A nominal $295 \mathrm{~L}$ tank located in Tank Pit 7 and is used primarily for the stripping of the "loaded" (pregnant) F-115 organic extraction stage organic. T-77 is the largest of the non-waste processing tanks. T-77 and its outlet lines are constructed of HC. T-77 inlet lines are constructed of Zr.

b. Foxboro pneumatic instrument-T-77's liquid level and density are read out and recorded on an original Foxboro pneumatic instrument (over 40 years old). This instrument is typically reliable and is routinely calibrated. Broken polyethylene lines supplying the pneumatic signals to the transmitters and instrument can present some problems, but in recent years, UV-resistant polyethylene has decreased potential breaking of lines due to age and exposure to UV light. Plugged lines can be a problem if the plug cannot be removed easily.

c. T-77 sparger-An electric switch activates a solenoid that opens and allows air to flow into a deep leg in the tank for solution mixing in the tank. The air flow into the tank is regulated by an orifice plate in the sparge line. The orifice plate can plug requiring the suspension of sparging operations in T77. The orifice plate would have to be removed and cleaned. This typically only requires no more than $4 \mathrm{~h}$. Plugs in the sparge line downstream of the orifice or flow meter can occasionally occur, and this can sometimes require as many as $12-24 \mathrm{~h}$ to clear. The sparger solenoid has failed, even though failure is not frequent. Rebuilds and replacements are readily available and cause very minimal down time for repairs. If necessary an alternate method of sparging T-77 is by using the in-cell air and applying air pressure down the T-77 sample uptake line.

d. T-77 processing lines - These are the lines that are used to transfer process solutions into and out of T-77. Typically, these lines are not an issue, though at times some lines are preferred over others, and plugged lines can be a problem if the plug cannot be removed easily or an alternate line cannot be used.

e. T-77 sampler-A $1 \mathrm{~L}$ glass bottle with a 2-line rubber stopper for sampling in Cubicle 6 is connected to a 1-line polyethylene block installed in the T-77 needle block position (SA-T77) on BR-7 using polyethylene tubing. Glass bottles, stoppers, polyethylene tubing with female ball joints, polyethylene tubing, and plastic compression fittings are kept in stock and typically readily available. (The designed sample technique is no longer employed because there are no needle blocks available to use the vacuum accumulators to pull samples from the tank pit tanks.)

9.2.6 F-115 CLEANEX Extraction Raffinate Transfer from T-77 and F-115 Heel Check 
The aqueous raffinate (flush solution) collected in T-77 from F-115 is transferred back from T-77 into F-115 and acidified and sampled in F-115 to confirm that no residual recoverable material was left in F-115 or lost in aqueous flushes to T-77.

Cell equipment utilized:

a. T7707 GN in Cubicle 7 right DC well-Refer to Section 9.2.8.a for discussion of T7707 GN.

b. T73402 GN on RR-7-Standard Ta GN (Refer to Section 1.6.1.d. for discussion of T73402 GN) with polyethylene line connected to T7707 GN.

c. PS-733 position on RR-7-Refer to Section 2.3.2.c for discussion of PS-733.

d. HV-T734-2 on RR-7-Refer to Section 1.6.1.f for discussion of HV-T734-2.

e. T-734 on RR-7-Refer to Section 1.6.1.g for discussion of T-734.

f. HV-T734-3 on RR-7-Refer to Section 1.6.1.h for discussion of HV-T7343.

g. HV-T734-1 on RR-7-Refer to Section 1.6.1.k for discussion of HV-T7341.

h. T73404 GN on RR-7-Standard Ta GN outlet from T-734 (Previously discussed in Section 1.6.1.i.) with polyethylene line connected to 1-line block at SA-F115 position.

i. 1-line block at SA-F115 position on BR-7-Specially fabricated block. Fabricated in house. Typically spares are available

j. KP-735on RR-7-Refer to Section 1.6.1.l for discussion of KP-735.

k. CU7AJ-In-cell air jet used for vacuum source. Previously discussed in Section 1.4.1.p.

l. Polyethylene tubing-For vacuum lines, pressure lines, demineralized water lines, and portions of the transfer route. Male ball joints and plastic compression fittings are readily available. Previously discussed in Section

\subsection{1.q.}

\subsubsection{F-115 CLEANEX Stripping}

Each strip is transferred (phase separated) from T-77 to T-60 and then from T-60 to T-604. Both T-60 and T-604 will contain a small volume of diluent (typically $10 \mathrm{~L}$ in $\mathrm{T}-60$ and $5 \mathrm{~L}$ in T-604) to prevent the transfer of any entrained pregnant organic to the strip collection tank. (The diluent is obtained for flushing by utilizing the M-703 vacuum/pressure transfer system previously discussed in Section 2.3.1.) From $T-604$, the strip solution is transferred to the strip collection tank (usually an evaporator like T-40). T-40 is evaporated as needed to make room for the required number of strips.

NOTE: Eight strips of the T-77 organic are programmed. Additional strips may be added to meet desired activity goals for stripping.

a. Strip solution is made up ahead of time in $\mathrm{M}-1, \mathrm{M}-2$, or both and sampled to confirm the acid concentration is in the prescribed limits. Refer to Sections

1.2.1 and 1.2.2 for discussion of M-1 and M-2, respectively. 
b. Each strip is added to T-77 from M-70 (or equivalent tank) via the T-77 decon line (V-T77-4). Refer to Section 2.1.4 for discussion of M-70.

c. Each strip is sparged and settled in T-77, and the eighth strip solution is sampled for activities. T-77 sampling is discussed in Section 9.2.5.e. Additional strips can be performed if necessary to meet the desired activity limits.

d. Once the strip sample results meet the desired activity limits, the organic phase in T-77 is sampled. T-77 sampling is discussed in Section 9.2.5.e.

\subsubsection{F-115 CLEANEX Strip Transfer (T-77 to T-60)}

The transfer route from T-77 to T-60 utilizes T-607 vacuum/pressure tank on BR-6 to perform the transfer. Refer to Sections 6.2.1 and 6.2.2 for discussion of T-60.

Cell equipment utilized:

a. T7707 GN in Cubicle 7 right DC well—Standard Zr GN outlet from T-77 with polyethylene line connected T60702 GN; fabricated in house. Typically spares are available.

b. T60702 GN on BR-6-Standard Zr GN with PVC ball valve and polyethylene line connected from T7707 GN; fabricated in house. Typically spares are available.

c. PS-607 position on BR-6-Phase separator with slide valve installed in position. Failure of a PS is rare. Failure of the O-rings on a slide valve is the typical failure mode. Replacement of the slide valve O-rings is accomplished rather quickly inside the hot cell cubicle. Spare replacement O-rings are kept in stock. The PSs and slide valves are fabricated in house and are limited to an adequate number of spare PSs that are available for replacement if a PS exhibits a catastrophic failure. Typically gaskets, O-rings, and glass tubes are available for rebuilding. Spare base plate assemblies, top plates, and slide valves may not be available, but these normally do not require replacement.

d. T-607on BR-6-3 in. diameter $\times 3 \mathrm{ft}$ tall flanged glass column located on BR-6. Over time the glass becomes dark from radiation exposure, and there have been a few incidents where the glass has broken. This requires removal of the tank from BR-6, removal from Cubicle 6 via the Transfer Case, moving the tank to the Decon Box for replacement of the glass and gaskets, returning the tank to Cubicle 6, and reinstalling into position on BR-6. This can require as many as $3-5$ working days to accomplish. In the past, very minor leaks around the flange gaskets, typically seen under vacuum, have occurred. Some limited success has been achieved by tightening the flange bolts that can be accessed while T-607 is still in position on BR-6. If the leaks cannot be stopped, then removal of T-607 to the Decon Box for repair is typically required. Spare components are usually available for repairs to T607. 
e. HV-T607 position on BR-6-Pass through polyethylene block; fabricated in house. Typically spares are available.

f. T60703 GN on BR-6 - Standard Zr GN outlet from T-607 with PVC ball valve and polyethylene line connected to T6002 GN; fabricated in house. Typically spares are available.

g. T6002 GN in Cubicle 6 left DC well-With polyethylene line connected from T60703 GN. Previously described in Section 6.2.3.c.

h. T-60-Interim receiving tank for F115 CLEANEX strip solution. Refer to Sections 6.2.1 and 6.2.2 for discussion of T-60.

i. KP-607 on BR-6-A KOP for the T-607 vacuum/pressure transfer system. Typically gaskets, O-rings, and glass tubes are available for rebuilding. Spare base and top plates may not be available, but these normally do not require replacement.

j. CU6AJ_-In-cell air jet used for vacuum source. Typically spares are available.

k. Polyethylene tubing-For vacuum lines, pressure lines, demineralized water lines, and portions of the transfer route. Male ball joints and plastic compression fittings are readily available.

\subsubsection{F-115 CLEANEX Strip Transfer (T-60 to T-604)}

The transfer route from T-60 to T-604 utilizes T-734 vacuum/pressure tank on RR-7 to perform the transfer. Refer to Sections 2.3.8 and 2.3.9 for discussion of T-604.

Cell equipment utilized:

a. T6007 GN in Cubicle 6 left DC well—Standard Ta GN outlet from T-60 with polyethylene line connected T73402 GN position on RR-7.

b. T73402 GN on RR-7-Standard Ta GN (Previously discussed in Section 1.6.1.d.) with polyethylene line connected to T6007 GN.

c. PS-733 position on RR-7-Refer to Section 2.3.2.c for discussion of PS-733.

d. HV-T734-2 position on RR-7-Refer to Section 1.6.1.f for discussion of HV-T734-2.

e. $\quad$ T-734 on RR-7-Refer to Section 1.6.1.g for discussion of T-734.

f. HV-T734-3 position on RR-7-Refer to Section 1.6.1.h for discussion of HV-T734-3.

g. HV-T734-1 position on RR-7-Refer to Section 1.6.1.k for discussion of HV-T734-1.

h. T73404 GN on RR-7-Standard Ta GN outlet from T-734 (Previously discussed in Section 1.6.1.i.) with polyethylene line connected to C7405 GN.

i. C7405 GN-Located in Cubicle 7 left DC well, standard Ta GN inlet into T604. Previously discussed in Section 2.3.7.h.

j. KP-735on RR-7-Refer to Section 1.6.1.l for discussion of KP-735.

k. CU7AJ-In-cell air jet used for vacuum source. Previously discussed in Section 1.4.1.p. 
l. Polyethylene tubing — For vacuum lines, pressure lines, demineralized water lines, and portions of the transfer route. Male ball joints and plastic compression fittings are readily available. Previously discussed in Section

\subsection{1.q.}

\subsubsection{F-115 CLEANEX Strip Transfer (T-604 to T-40)}

Once the strip solution is received into T-604, each strip is sparged and settled in T-604. Each strip is transferred (phase separated) from T-604 to a strip collection tank (usually an evaporator like T-40). The transfer route from T-604 to T-40 utilizes P-653 vacuum/pressure diaphragm pump on BR-6 to perform the transfer.

Cell equipment utilized:

a. 7PDV-604-1 (Type D set to 7) on BR-6-Refer to Section 2.3.10.a for discussion of 7PDV-604-1.

b. Phase Separator PS-604 on BR-6-Refer to Section 2.3.10.b for discussion of PS-604.

c. 2-line block at HCV-P604-1 position on BR-6-With PVC ball valve and polyethylene line connected from left side to P65301 GN Refer to Section 2.3.10.c for discussion of HCV-P604-1.

d. P65301 GN on BR-6-Standard Zr GN (Previously discussed in Section

2.3.10.d.) with polyethylene line connected from left side of a 2-line block at HCV-P604-1.

e. P-653 on BR-6-Previously discussed in Section 2.3.10.e.

f. P65302 GN on BR-6-With PVC valve and polyethylene line connected to special FDV at FDV-T604 (line at top) on BR-6.

g. Special FDV-T604 (line at top) on BR-6-With polyethylene line to C4C601 GN. Refer to Section 2.3.10.g for discussion of FDV-T604.

h. C4C601 GN in Cubicle 6 left DC-Standard Zr GN to jumper between Cubicle 6 DC well and Cubicle 4 DC well. Previously discussed in Section 2.3.2.h.

i. C4C601 jumper-Previously discussed in Section 2.3.2.i.

j. $\quad$ C4C601 jumper-Previously discussed in Section 2.3.2.j.

k. C4C601 GN on BR-4-Standard Zr GN with polyethylene line connected to T4003 GN in Cubicle 4 left DC well. Previously discussed in Section 2.3.2.k.

1. T4003 GN in Cubicle 4 left DC well—Standard Zr GN (teed with T4013 line in Cubicle 6 left DC well). Previously discussed in Section 3.6.2.l.

m. T4013 GN in Cubicle 6 left DC well-Teed to T4003 line in Cubicle 4 left DC well) has a male plug installed at the DC position in the well. Previously discussed in Section 3.6.2.m.

n. 7PDV-604-2 (Type D set to 2) on BR-6-Previously described in Section 2.3.10.g above. 
o. 2-line block at HCV-P604-2 position on BR-6-With PVC valve and polyethylene line connecting the two lines. Previously described in Section 2.3.10.h above.

p. Polyethylene tubing — For vacuum lines, pressure lines, and demineralized water lines, and portions of the transfer route. Male ball joints, and plastic compression fittings are readily available. Previously described in Section 1.4.1.q above.

\subsubsection{F-115 CLEANEX Strip Product Collection Tank, Evaporator T-40}

T-40 has several systems that are required to operate properly to maintain functionality. Typically, tank pit tanks like T-40 do not have many physical or mechanical issues that interrupt routine operations. Loss of steam and cooling water capabilities could have a negative impact, but this is a low probability.

a. T-40-A nominal 63 L HC shell with Ta liner evaporator tank located in Tank Pit 4 and is used to collect product and feed solutions for volume reduction. T-40 has an annulus filled with $\mathrm{Hg}$ for increased heat transfer capability and is used to evaporate the dissolved actinide product from the target rods as part of the hot feed adjustment for anion exchange operations.

b. Foxboro pneumatic instrument-T-40's liquid level and density are read out and recorded on an original Foxboro pneumatic instrument (over 40 years old). This instrument is typically reliable and is routinely calibrated. T-40 pressure is read out on an original Foxboro pneumatic instrument (also over 40 years old) that provides read outs for several tank pressures. Specific tank pressures provided on this instrument are selected for read out by a selector switch. Broken polyethylene lines supplying the pneumatic signals to the transmitters and instrument can present some problems, but in recent years, UV-resistant polyethylene has decreased potential breaking of lines due to age and exposure to UV light. Plugged lines can be a problem if the plug cannot be removed easily.

c. T-40 sparger-An electric switch activates a solenoid that opens and allows air to flow into a deep leg in the tank for solution mixing in the tank. The air flow into the tank is regulated by an orifice plate in the sparge line. The orifice plate can plug requiring the suspension of sparging operations in $\mathrm{T}$ 40. The orifice plate would have to be removed and cleaned. This typically only requires no more than $4 \mathrm{~h}$. Plugs in the sparge line downstream of the orifice or flow meter can occasionally occur. This can sometimes require as many as $12-24 \mathrm{~h}$ to clear. The sparger solenoid has failed, even though failure is not frequent. Rebuilds and replacements are readily available and cause very minimal down time for repairs. If necessary an alternate method of sparging T-40 is by using the in-cell air and applying air pressure down the T-40 sample uptake line.

d. T-40 processing lines-These are the lines that are used to transfer process solutions into and out of T-40. Typically, these lines are not an issue, though 
at times some lines are preferred over others, and plugged lines can be a problem if the plug cannot be removed easily or an alternate line cannot be used.

e. T-40 sampler-A $1 \mathrm{~L}$ glass bottle with a 2-line rubber stopper for sampling in Cubicle 4 is connected to a polyethylene line that is connected to a GN installed in the T4002 position in the Cubicle 4 right disconnect well. The T4002 jumper has been removed due to the T4002 line on the sample rack leaking. Glass bottles, stoppers, polyethylene tubing with female ball joints, polyethylene tubing, and plastic compression fittings are kept in stock and typically readily available. (The designed sample technique is no longer employed since T-40 vacuum accumulator and needle block position [SAT43] are out of service.)

\subsubsection{T-40 Heating/Cooling}

a. Thermocouples-For monitoring the temperature during evaporation and feed adjustment. The thermocouples come off the tank in the tank pit and then run through the LAA wall and eventually go to the Control Room, where they read out on temperature instruments. The thermocouples are typically very reliable, but thermocouple failure necessitating thermocouple replacement is very difficult and time-consuming. Instrument failure could potentially cause significant delays as well. Thermocouple or temperature instrument failure would prevent use of T-40 as an evaporator.

b. T-40 steam jacket and associated steam and condensate lines and valvesUsed for heating the tank for evaporation. If the steam supply to the evaporator is shut down for any reason, T-40 cannot be evaporated. Manual valves that allow RCW or steam to flow through the heating/cooling jacket on T-40 are located in the MUA. There is also a solenoid valve on the condensate discharge from T-40 that is operated by an electrical switch in the Control Room. Typically, replacement parts are available for these valves, and most repairs are accomplished in less than $4 \mathrm{~h}$.

c. PIC-T40 - The steam supply to T-40 is controlled at the main panel board in the Control Room by pressure indicator controller (PIC-T40), which supplies air pressure to a steam regulator, pressure control valve (PCV-T40), on the steam supply line to the T-40 steam jacket. Typically, the PICs are very reliable and rarely need repair or replacement. The PCVs can sometimes have diaphragm failures and leaks that require repair and occasional replacement. This can take up to $24 \mathrm{~h}$ depending on the extent of the repair.

d. T-40 RCW lines and valves-Used for cooling the tank. The RCW system supplies cooling water to T-40. Manual valves that allow RCW or steam to flow through the heating/cooling jacket on T-40 are located in the MUA. Typically, replacement parts are available for theses valves, and most repairs are accomplished in less than $4 \mathrm{~h}$.

e. Condenser on the VOG line-The H-40 condenser is located in the tank pit and has RCW supplied to it for condensing the vapor in the condensate line. 
The RCW supply to H-40 is controlled by manual valves located in the MUA. The operability of the condenser is very reliable. Spare parts are readily available for these valves, and most repairs are accomplished in less than $4 \mathrm{~h}$.

f. VOG-Used for ventilating the tank. During evaporation, vapor travels through condensers and the vapor is condensed and collected in a condensation collection tank. This line is intact, and no known reliability issues exist.

\subsubsection{F-115 CLEANEX Confirming Strip in T-60}

a. Once the stripping of T-77 is confirmed complete from the final T-77 strip sample analysis, a confirming strip (flush) is added to T-60 from M-70 or equivalent tank (M-704 in this case). Refer to Section 1.4.2 for a discussion of M-704.

b. The confirming flush is sparged, settled, and sampled in T-60. T-60 sampling is discussed in Section 6.2.1.e.

c. The confirming flush is transferred from T-60 to T-604 as described in Section 9.2.9.

d. Once the confirming flush sample analysis is determined to meet the activity specification to terminate stripping, the organic phase in T-60 is sampled. T60 sampling is discussed in Section 6.2.1.e.

e. Volume reductions are routinely performed during the stripping operations. Refer to Sections 9.2.11 and 9.2.12 below for a discussion of the Tank T-40 evaporator.

\subsubsection{F-115 CLEANEX Confirming Strip in T-604}

a. Once the stripping of T-77 is confirmed complete from the final T-77 strip sample analysis and the confirming strip in T-60 is confirmed complete from the analysis, a confirming strip (flush) is added to T-604 from M-70 or equivalent tank (M-704 in this case).

b. The confirming flush is sparged, settled, and sampled in T-604. T-604 sampling is discussed in Section 2.3.8.e.

c. The confirming flush is transferred from T-604 to T-40 as described in Section 9.2.10.

d. Once the confirming flush sample analysis is determined to meet the activity specification to terminate stripping, the organic phase in T-604 is sampled. T-604 sampling is discussed in Section 2.3.8.e.

e. Volume reductions are routinely performed during the stripping operations. Refer to Sections 9.2.11 and 9.2.12 below for a discussion of the Tank T-40 evaporator.

\subsubsection{CLEANEX Organic Cleanout}


Once stripping operations have been confirmed complete based on T-77, T-60, and T-604 sample results, organic cleanouts of these tanks may be performed.

a. Addition of a specified volume of low concentration $(<5 M) \mathrm{NaOH}$, is made to T-77, T-60, and T-604 to neutralize the acidic aqueous heel and acidic organic for transfer to F-126 waste tank via routes already discussed. Refer to Sections 9.2.5 (T-77), 6.2.1 and 6.2.2 (T-60), and 2.3.8 and 2.3.9 (T-604).

b. The tanks are sparged and sampled to confirm that the solutions are neutralized or slightly basic. T-77, T-60, and T-604 sampling are discussed in Section 9.2.5.e, Section 6.2.1.e, and Section 2.3.8.e, respectively.

c. T-77, T-60, and T-604 contents are transferred to F-126 utilizing T-734 vacuum/pressure tank on RR-7 to perform the transfer. The transfer routes are discussed below in Sections 9.2.16, 9.2.17, and 9.2.18, respectively.

d. T-77, T-60, and T-604 are flushed to F-126 with diluent and water using the T-734 vacuum/pressure tank on RR-7 to perform the transfer. The diluent is obtained for the flushing by utilizing the M-703 vacuum/pressure transfer system previously discussed in Section 2.1.3.

e. At times when the waste jets from T-77 (J-T77A), T-60 (J-T60), and T-604 (J-T604) to F-115 waste tank are operating, water flushes are added to T-77, T-60, and T-604, sparged, and jetted to F-115. Otherwise the flushes are transferred to F-126 via the routes outlined in Sections 9.2.16, 9.2.17, and 9.2.18, respectively. If there is a steam outage, then the steam jets cannot be utilized for transfers. Steam jets can fail and since they are located in the Hot Cell Tank Pits, it is very difficult to replace them, even with the availability of spare jets. At times, the jets can get plugged with debris and it can require significant effort to unplug it causing operational delays. This is why the route to F-126 waste tank is often utilized.

f. Any remaining diluent in T-60 and T-604 is steam distilled by adding water and evaporating off a specified amount of water to T-34, the evaporator condensate collection tank. (T-60 evaporator operation is discussed in

Sections 6.2.1 and 6.2.2. T-604 evaporator operation is discussed in Sections 2.3.8 and 2.3.9. The process condensate receiving tank, T-34, is discussed in Section 9.5.)

g. The remainder of the water in the tanks after the steam distillation is complete is jetted to F-115 or transferred to F-126 per previously discussed routes.

\subsubsection{F-115 CLEANEX T-77 Waste Organic Transfer}

Once T-77 has been confirmed to be basic (or neutral) based on T-77 sample results, transfer of the waste organic may be performed.

NOTE: The transfer route from T-77 to F-126 using T-734 is discussed again below in Sections 9.2.16.a-k.

Cell equipment utilized: 
a. T7707 GN in Cubicle 7 right DC well—Standard Zr GN outlet from T-77 (Previously discussed in Section 9.2.6.a) with polyethylene line connected T73402 GN position on RR-7.

b. T73402 GN on RR-7-Standard Ta GN (Section 1.6.1.d) with polyethylene line connected to T7707 GN

c. PS-733 on RR-7-Refer to Section 1.6.1.e for discussion of PS-733.

d. HV-T734-2 position on RR-7-Refer to Section 1.6.1.f for discussion of HV-T734-2.

e. T-734 on RR-7-Refer to Section 1.6.1.g for discussion of T-734.

f. HV-T734-3 position on RR-7-Refer to Section 1.6.1.h for discussion of HV-T734-3.

g. HV-T734-1 position on RR-7-Refer to Section 1.6.1.k for discussion of HV-T734-3.

h. T73404 GN on RR-7-Standard Ta GN outlet from T-734 (Previously discussed in Section 1.6.1.i) with polyethylene line connected to 1-line block at SA-F126 position.

i. 1-line polyethylene block at SA-F126 needle block position on BR-6-Refer to Section 9.2.16.i for discussion of 1-line polyethylene block at SA-F126 position.

j. $\quad$ KP-735 on RR-7-Refer to Section 1.6.1.I for discussion of KP-735.

k. CU7AJ-in-cell air jet used for vacuum source. Previously discussed in Section 1.4.1.p.

l. Polyethylene tubing - For vacuum lines, pressure lines, demineralized water lines, and portions of the transfer route. Male ball joints and plastic compression fittings are readily available. Previously discussed in Section

\subsection{1.q.}

9.2.17 F-115 CLEANEX T-60 Waste Organic Transfer

Once T-60 has been confirmed to be basic (or neutral) based on T-60 sample results, transfer of the waste organic may be performed.

NOTE: The transfer route from T-60 to F-126 using T-734 is discussed below in Sections 9.2.17.a-k.

Cell equipment utilized:

a. T6007 GN in Cubicle 6 left DC well-Standard Ta GN outlet from T-60 (Previously discussed in Section 9.2.9.a.) with polyethylene line connected T73402 GN position on RR-7.

b. T73402 GN on RR-7-Standard Ta GN (Previously discussed in Section 1.6.1.d.) with polyethylene line connected to T6007 GN.

c. PS-733 on RR-7-Refer to Section 1.6.1.e for discussion of PS-733.

d. HV-T734-2 position on RR-7-Refer to Section 1.6.1.f for discussion of HV-T734-2.

e. T-734 on RR-7-Refer to Section 1.6.1.g for discussion of T-734. 
f. HV-T734-3 position on RR-7-Refer to Section 1.6.1.h for discussion of HV-T734-3.

g. HV-T734-1 position on R-Refer to Section 1.6.1.k for discussion of HVT734-1.

h. T73404 GN on RR-7-Standard Ta GN outlet from T-734 (Previously discussed in Section 1.6.1.i.) with polyethylene line connected to 1-line block at SA-F126 position.

i. $\quad$ 1-line polyethylene block at SA-F126 needle block position on BR-6-Refer to Section 9.2.16.i for discussion of 1-line polyethylene block at SA-F126 position.

j. $\quad$ KP-735 on RR-7-Refer to Section 1.6.1.l for discussion of KP-735.

k. CU7AJ-In-cell air jet used for vacuum source. Previously discussed in Section 1.4.1.p.

l. Polyethylene tubing - For vacuum lines, pressure lines, demineralized water lines, and portions of the transfer route. Male ball joints and plastic compression fittings are readily available. Previously discussed in Section

\subsection{1.q.}

9.2.18 T-604 CLEANEX Waste Organic Transfer

Once T-604 has been confirmed to be basic (or neutral) based on T-604 sample results, transfer of the waste organic may be performed.

NOTE: The transfer route from T-604 to F-126 using T-734 is discussed below in Sections 9.2.18.a-l.

Cell equipment utilized:

a. 7PFDV-604-1 (Type D set to 7) on BR-6-Fabricated in house. Typically spares are available.

b. Special 2-line block at PS-604 position on BR-6-With polyethylene tubing connected to the left side and to T73402 GN; fabricated in house. Typically spares are available. Previously discussed in Section 2.3.17.b.

c. T73402 GN on RR-7—Standard Ta GN (Previously discussed in Section 1.6.1.d.) with polyethylene line connected to the left side of special 2-line block as PS-604 position on BR-6.

d. PS-733 on RR-7-Refer to Section 1.6.1.e for discussion of PS-733.

e. HV-T734-2 position on RR-7-Refer to Section 1.6.1.f for discussion of HV-T734-2.

f. T-734 on RR-7-Refer to Section 1.6.1.g for discussion of T-734.

g. HV-T734-3 position on RR-7-Refer to Section 1.6.1.h for discussion of HV-T734-3.

h. HV-T734-1 position on RR-7-Refer to Section 1.6.1.k for discussion of HV-T734-1. 
i. $\quad$ T73404 GN on RR-7-Standard Ta GN outlet from T-734 (Previously discussed in Section 1.6.1.i.) with polyethylene line connected to 1-line block at SA-F126 position.

j. 1-line polyethylene block at SA-F126 needle block position on BR-6-Refer to Section 9.2.16.i for discussion of 1-line polyethylene block at SA-F126 position.

k. KP-735 on RR-7-Refer to Section 1.6.1.I for discussion of KP-735.

l. CU7AJ-In-cell air jet used for vacuum source. Previously discussed in Section 1.4.1.p.

m. Polyethylene tubing — For vacuum lines, pressure lines, demineralized water lines, and portions of the transfer route. Male ball joints and plastic compression fittings are readily available. Previously discussed in Section

\subsection{1.q.}

\subsubsection{F-115 CLEANEX Product Tank Sampling in T-40:}

a. Once the F-115 CLEANEX stripping operations are complete and the final confirming strip has been transferred into T-40, T-40 is sampled at a high volume. Refer to Section 9.2.11.e for discussion of T-40 sampler.

b. T-40 is typically evaporated to a prescribed volume in preparation for the next process operation. Refer to Sections 9.2.11 and 9.2.12 for discussion of T-40 evaporator details.

\subsection{F-126 Waste Tank Operations}

\subsubsection{Final Waste Collection, Tank F-126}

F-126 is located in the waste tank pit along with F-111 and F-115. Typically, F126 receives aqueous waste from F-115, organic waste from various process tanks as a result of organic cleanout operations, and from L-131 solution change outs approximately twice per month. Occasionally, smaller volumes of waste may be transferred to F-126 from other miscellaneous sources in the hot cells. F-126 has several systems that are required to operate properly to maintain functionality. Typically F-126 does not have many physical or mechanical issues that interrupt routine operations. Plugging instrument probes or broken instrument lines can pose some minor problems, but these problems are a low probability.

a. Tank F-126 - A nominal 3,531 L tank constructed of stainless steel with probes constructed of stainless steel. F-126 is located in the waste tank pit.

b. Foxboro pneumatic instrument-F-126 only has a liquid level read out that is recorded on an original Foxboro pneumatic instrument (over 40 years old). This instrument is typically reliable and is routinely calibrated. Broken polyethylene lines supplying the pneumatic signals to the transmitters and instrument can present some problems, but in recent years, UV-resistant polyethylene has decreased potential breaking of lines due to age and 
exposure to UV light. Plugged lines can be a problem if the plug cannot be removed easily.

c. F-126 sparger-An electric switch activates a solenoid that opens a valve, HCV-F126-6, and allows air to flow into a deep leg in the tank for solution mixing in the tank. The air flow into the tank is regulated by an orifice plate in the sparge line. The orifice plate can plug requiring the suspension of sparging operations in F-126. The orifice plate would have to be removed and cleaned. This typically only requires no more than $4 \mathrm{~h}$. Plugs in the sparge line downstream of the orifice or flow meter can occasionally occur, and this can sometimes require as many as $12-24 \mathrm{~h}$ to clear. The sparger solenoid has failed, even though failure is not frequent. Rebuilds and replacements are readily available and cause very minimal down time for repairs. If necessary an alternate method of sparging F-126 is by using the incell air and applying air pressure down the F-126 sample uptake line.

d. F-126 processing lines-These are the lines that are used to transfer process solutions into and out of F-126. Typically, these lines are not an issue, though at times some lines are preferred over others, and plugged lines can be a problem if the plug cannot be removed easily or an alternate line cannot be used.

e. F-126 sampler-A $1 \mathrm{~L}$ glass bottle with a 2-line rubber stopper for sampling in Cubicle 7 is connected to a 1-line polyethylene block installed in the F-126 needle block position (SA-F126) on BR-7 using polyethylene tubing. Glass bottles, stoppers, polyethylene tubing with female ball joints, polyethylene tubing, and plastic compression fittings are kept in stock and typically readily available. (The designed sample technique is no longer employed because there are no needle blocks available to use the vacuum accumulators to pull samples from the tank pit tanks.)

\subsubsection{F-126 waste solution transfer}

a. Typically, when the volume in F-126 reaches $\sim 80 \%-85 \%$ of the maximum volume of the tank, the waste solution in F-126 is sparged and sampled for disposal per the "Disposal of F-126 Contents to Melton Valley MCS [Monitoring Control Station] Waste Tank F-1800” procedure. The sample is submitted for radiochemical analysis for disposal. Refer to Section 9.3.1.e for discussion of F-126 sampler.

b. At this point F-126 is taken out of normal waste receiving service.

c. The REDC Liquid Waste Generator Interface Equivalent makes an official request to the ORNL Liquid and Gaseous Waste Operations (LGWO) to schedule the transfer of waste solution from F-126 to the F-1800 waste tank located at Building 7966, the Monitoring and Control Station for Melton Valley.

d. Once the results are received, they are reviewed by the REDC Liquid Waste Generator Interface Equivalent. The results have to be approved by the 
Liquid Waste Generator Interface Equivalent and meet the agreed upon limits from ORNL LGWO to make the waste transfer from F-126.

e. The waste line from F-126 to F-1800 was replaced in 1996/1997. It is a double containment line that has redundant systems that are monitored at the Monitoring and Control Station by the ORNL LGWO to ensure the integrity of the waste line.

f. The REDC Hot Cell Operations technicians perform the operations in the F126 disposal procedure and perform checks on the F-126 waste transfer system prior to performing the waste transfer as well.

g. Natural Th is added to F-126 to poison the fissile isotopes of $\mathrm{Pu}$ that may be present in F-126. Typically, $100 \mathrm{~g}$ of Th in the form of $\mathrm{Th}\left(\mathrm{NO}_{3}\right)_{4} 4 \mathrm{H}_{2} \mathrm{O}$ is added to F-126 for each waste transfer to F-1800. If more than $0.5 \mathrm{~g}$ of fissile isotopes of $\mathrm{Pu}$ is present, then additional Th is required to be added. The Th solution is prepared in $1 \mathrm{~L}$ polyethylene bottles, bagged into the hot cell cubicles, transferred to Cubicle 7, and then transferred to F-126 via the F12602 sample line.

h. F-126 is confirmed to be basic from the sample obtained per the waste disposal procedure. If F-126 is not basic, $\mathrm{NaOH}$ must be added and flushed to F-126 from M-40. Refer to Section 9.6 for discussion of M-40.

i. Coordination for the actual disposal operations is done between the REDC Hot Cell Operations personnel and the Waste Operations Control Center operated by ORNL LGWO personnel.

j. On occasion, the steam jet J-126B that is used to transfer the waste solution is temperamental and overheats and will not jet. It has to be cooled by adding water back down through the water flush line to cool it down.

k. In the past, there have been equipment failures that have delayed the waste transfer. The monitoring system has many electronic components, and there have been some noted failures. Some of these failures have caused a delay in making a transfer of waste from F-126 to F-1800. These delays have been as long as several weeks. In recent years, these delays have been minimal and infrequent.

l. The waste line is flushed to F-1800 with 175 gal of water at the end of the transfer.

m. Final volumes are recorded for F-126 (should be empty or very near empty) and F-1800 and F-126 is placed back into normal service.

\subsection{F-111 Waste Tank Operations}

\subsubsection{Waste Collection, Tank F-111}

F-111 is located in the waste tank pit along with F-115 and F-126. Typically, F111 receives dissolved $\mathrm{Al}$ solution from HFIR target rod processing operations or Mark 42 segment process operations. If needed F-111 can receive other solutions, but the solutions must be basic prior to being transferred into F-111 because F-111 is a stainless steel tank. F-111 has several systems that are 
required to operate properly to maintain functionality. Typically, F-111 does not have many physical or mechanical issues that interrupt routine operations. Plugging instrument probes or broken instrument lines can pose some minor problems, but these problems are a low probability.

a. Tank F-111-A nominal 1,800 L tank constructed of stainless steel with probes constructed of stainless steel. F-111 is located in the waste tank pit.

b. Yokogawa pneumatic instrument-F-111 has a liquid level and density read out that is recorded on a Yokogawa electronic instrument ( 15 years old). This instrument is typically reliable and is routinely calibrated. Broken polyethylene lines supplying the pneumatic signals to the transmitters and instrument can present some problems, but in recent years, UV-resistant polyethylene has decreased potential breaking of lines due to age and exposure to UV light. Plugged lines can be a problem if the plug cannot be removed easily.

c. F-111 sparger - An electric switch activates a solenoid that opens a valve, HCV-F111-6, and allows air to flow into a deep leg in the tank for solution mixing in the tank. The air flow into the tank is regulated by an orifice plate in the sparge line. The orifice plate can plug requiring the suspension of sparging operations in F-111. The orifice plate would have to be removed and cleaned. This typically only requires no more than $4 \mathrm{~h}$. Plugs in the sparge line downstream of the orifice or flow meter can occasionally occur, and this can sometimes require as many as $12-24 \mathrm{~h}$ to clear. The sparger solenoid has failed, even though failure is not frequent. Rebuilds and replacements are readily available and cause very minimal down time for repairs. If necessary an alternate method of sparging F-111 is by using the incell air and applying air pressure down the F-111 sample uptake line.

d. F-111 processing lines-These are the lines that are used to transfer process solutions into and out of F-111. Typically, these lines are not an issue, though at times some lines are preferred over others, and plugged lines can be a problem if the plug cannot be removed easily or an alternate line cannot be used.

e. F-111 sampler-A $1 \mathrm{~L}$ glass bottle with a 2-line rubber stopper for sampling in Cubicle 7 is connected to a 1-line polyethylene block installed in the F-111 needle block position (SA-F111) on BR-7 using polyethylene tubing. Glass bottles, stoppers, polyethylene tubing with female ball joints, polyethylene tubing, and plastic compression fittings are kept in stock and typically readily available. (The designed sample technique is no longer employed because there are no needle blocks available to use the vacuum accumulators to pull samples from the tank pit tanks.)

\subsection{Process Condensate Collection Tank, Tank T-34}

9.5.1 T-34 is the central collection tank for the condensate generated from volume reduction and feed adjustment evaporations for the many process operations 
associated with the HFIR target rod processing for the ${ }^{252} \mathrm{Cf}$ Production Program. $\mathrm{T}$-34 has several systems that are required to operate properly to maintain functionality. Typically, tank pit tanks like T-34 do not have many physical or mechanical issues that interrupt routine operations. Plugging instrument probes or broken instrument lines can pose some minor problems, but these problems are a low probability and typically require minimal time and manpower to correct. Due to the potential for mixed acids to be present in the condensate collected in T-34, a caustic ( $\mathrm{NaOH}$ ) heel is added to T-34 from M-40 to neutralize any acid condensate. Refer to Section 9.6 for discussion of M-40.

a. Tank T-34-A nominal 153.3 L tank constructed of HC with HC probes and is located in Tank Pit 3.

b. Foxboro pneumatic instrument-T-34's liquid level and density are read out and recorded on an original Foxboro pneumatic instrument (over 40 years old). This instrument is typically reliable and is routinely calibrated. Broken polyethylene lines supplying the pneumatic signals to the transmitters and instrument can present some problems, but in recent years, UV-resistant polyethylene has decreased potential breaking of lines due to age and exposure to UV light. Plugged lines can be a problem if the plug cannot be removed easily.

c. T-34 sparger-An electric switch activates a solenoid that opens and allows air to flow into a deep leg in the tank for solution mixing in the tank. The air flow into the tank is regulated by an orifice plate in the sparge line. The orifice plate can plug requiring the suspension of sparging operations in $\mathrm{T}$ 34. The orifice plate would have to be removed and cleaned. This typically only requires no more than $4 \mathrm{~h}$. Plugs in the sparge line downstream of the orifice or flow meter can occasionally occur, and this can sometimes require as many as $12-24 \mathrm{~h}$ to clear. The sparger solenoid has failed, even though failure is not frequent. Rebuilds and replacements are readily available and cause very minimal down time for repairs. If necessary an alternate method of sparging T-34 is by using the in-cell air and applying air pressure down the T-34 sample uptake line.

d. T-34 processing lines-These are the lines that are used to transfer process solutions into and out of T-34. Typically, these lines are not an issue, though at times some lines are preferred over others, and plugged lines can be a problem if the plug cannot be removed easily or an alternate line cannot be used.

e. T-34 sampler-A $1 \mathrm{~L}$ glass bottle with a 2-line rubber stopper for sampling in Cubicle 6 is connected to a 1-line polyethylene block installed in the T-34 needle block position (SA-T34) on BR-6 using polyethylene tubing. Glass bottles, stoppers, polyethylene tubing with female ball joints, polyethylene tubing, and plastic compression fittings are kept in stock and typically readily available. (The designed sample technique is no longer employed because 
there are no needle blocks available to use the vacuum accumulators to pull samples from the tank pit tanks.)

\subsection{Cold Chemical Addition Tank, M-40}

M-40 is located in the MUA and is hard piped for making cold chemical additions to the Process Condensate Collection Tank T-34 and waste tank pit tanks, F-111, F-115, and F126.

9.6.1 M-40 is a chemical addition tank used for the addition of acids, bases, water, and other miscellaneous cold chemicals to T-34, F-111, F-115, and F-126. M-40 originally was a Penton lined and coated tank and was replaced with an in house, fabricated PVC tank with Hayward PVC ball valves on all the PVC inlet and outlet lines. M-40 was the first of the Penton lined and coated tanks in the MUA to be replaced by a PVC tank. M-40 is a nominal $50 \mathrm{~L}$ volume tank. The tank itself should last many years with no required maintenance. The ball valves sometimes develop leaks, but typically they are very reliable. Normally, leaking valves can be repaired, but replacement is usually about as cost-effective as repairing. The lines and valves associated with the tank are listed below:

a. V-M40-1, vent line to VOG

b. V-M40-2, solution addition line through the funnel

c. V-M40-3, 5 psig air pressure supply line

d. V-M40-4, outlet line/drain

e. V-M40-6, inlet from M-500

f. V-M40-7, outlet line to header for T-34 and waste tank header (for F-111, F-115, and F-126)

g. V-M40-8, outlet line from header to T-34

h. V-M40-10, sight glass valve at bottom of sight glass

i. V-M40-13, outlet line

j. V-M40-14, outlet line from header to waste tank header (to F-111, F-115, and F-126)

\subsection{Miscellaneous Process Operations}

\subsection{Miscellaneous Flushing}

During the course of performing the many process operations required to process, separate, and purify the ${ }^{252} \mathrm{Cf}$ product from the HFIR target rods, various amounts of recoverable material is lost through leaks from equipment failures and spillage of material during difficult handling operations. Also, some material can be held up inside the process equipment and piping. This material can add up and become a significant quantity that warrants recovery. Various systems, equipment, and specific locational flushing is performed to recover this material either routinely or specifically due to know material loses from spills or leaks.

10.1.1 External Equipment Flushing 
a. External equipment flushing is typically performed on a particular equipment rack located in the hot cell cubicles following a particular series of processing operations. At times some other individual equipment may be flushed that is independent of an equipment rack.

b. Usually, equipment racks are flushed externally with in-cell demineralized water. Unless some particular situation has occurred that would dictate it to be prudent to flush with an acid solution, then some localized area of an equipment rack might be flushed with a dilute $\mathrm{HNO}_{3}$ solution. The equipment racks should never be flushed down with an $\mathrm{HCl}$ solution. The final equipment flushing will always consist of water.

c. Other miscellaneous equipment that is utilized, but is not a part of an equipment rack, may be flushed down with water or an acid solution of specified concentration, if warranted.

d. Typically, if certain pieces of equipment are flushed with an acid solution, the type of acid is dependent on the composition of the equipment. Stainless steel can be flushed with a $\mathrm{HNO}_{3}$ solution, but is never flushed with an $\mathrm{HCl}$ solution.

e. The acid flushes are added from one of the MUA tanks and typically added down the appropriate cubicle solution addition line.

f. The external equipment flushes are allowed to drain to the cubicle floor and are typically transferred to a collection tank from the general hot cell cubicle floor area and sumps using one of the vacuum/pressure transfer systems located on one of the equipment racks in the hot cell cubicles. The flush solutions are normally collected in T-66 or T-79 tanks, where they are acidified (if needed), sparged, and sampled. Samples are submitted to CAS for analysis. Refer to Sections 10.2.1 and 10.2.2 for discussion of T-66 and $\mathrm{T}-79$, respectively.

g. The results of the analyses determines whether the solution contains adequate material to recover in a miscellaneous CLEANEX recovery stage in T-21 or T-23, or the solution is transferred to F-115 and handled there. Refer to Sections 2.3.3 and 2.3.4, respectively for discussion of T-21 and T-23. Refer to Section 9.2 for discussion of how waste solutions are managed in F-115.

\subsubsection{Internal Equipment Flushing}

a. Internal equipment flushing is periodically performed as a final processing step to ensure that any residual product material has been removed from the internal portions of the process equipment and piping. At times some other individual equipment may be flushed that is independent of an equipment rack.

b. Usually, equipment racks are flushed internally with acid solutions similar in concentration to the solutions used in the original process operations. Unless some particular situation has occurred that would dictate it to be prudent, then the equipment might be flushed with a different concentration of acid or even just water. The final equipment flushing will always consist of water. 
c. The internal equipment flushes are typically added from one of the MUA tanks down the appropriate line to the equipment to be flushed.

d. These equipment flushes are typically collected in tanks that have been associated with the preceding process operations, but some equipment flushes are collected in T-66 or T-79. The flush solution is typically acidified (if needed), sparged, and sampled. Samples submitted to CAS for analysis. Refer to Sections 10.2.1 and 10.2.2 for discussion of T-66 and T-79, respectively. Other collection tanks should have already been discussed as part of the original process operations discussion.

e. The results of the analyses determines whether the solution contains adequate material to recover in a miscellaneous CLEANEX recovery stage in T-21 or T-23, or the solution is transferred to F-115 and handled there. Refer to Sections 2.3.3 and 2.3.4, respectively for discussion of T-21 and T-23. Refer to Section 9.2 for discussion of how waste solutions are managed in F-115.

10.1.3 Hot Cell Cubicle Floor Flushing

a. Floor flushing can be done independent of any other flushing and is typically done with a dilute $\mathrm{HNO}_{3}$ solution, but a final floor flush consisting of water will always follow an external equipment rack flush down or a general floor flush operation.

b. Usually, water flushes following acid flushes of the floor areas in the cubicles are performed with in-cell demineralized water. Also, a water flush of the DC wells may also be performed in conjunction with prescribed external equipment rack of floor flush operation.

c. The floor and DC well flushes are typically transferred to a collection tank from the general hot cell cubicle floor area and sumps or DC wells using one of the vacuum/pressure transfer systems located on one of the equipment racks in the hot cell cubicles. The flush solutions are normally collected in T66 or T-79 tanks, where they are acidified (if needed), sparged, and sampled. Samples are submitted to CAS for analysis. Refer to Sections 10.2.1 and 10.2.2 for discussion of T-66 and T-79, respectively.

d. The results of the analyses determines whether the solution contains adequate material to recover in a miscellaneous CLEANEX recovery stage in T-21 or $\mathrm{T}-23$, or the solution is transferred to F-115 and handled there. Refer to Sections 2.3.3 and 2.3.4, respectively for discussion of T-21 and T-23. Refer to Section 9.2 for discussion of how waste solutions are managed in F-115.

\subsection{Miscellaneous Solution Collection Tanks, Tank T-66 and Tank T-79}

\subsubsection{Miscellaneous Solution Collection Tank, Tank T-66}

T-66 has several systems that are required to operate properly to maintain functionality. Typically, tank pit tanks like T-66 do not have many physical or mechanical issues that interrupt routine operations. Plugging instrument probes 
or broken instrument lines can pose some minor problems, but these problems are a low probability.

a. Tank T-66-A nominal 62.9 L tank constructed of Zr with Zr probes located in Tank Pit 6 and is typically used to collect miscellaneous solutions.

b. Foxboro pneumatic instrument-T-66's liquid level and density are read out and recorded on an original Foxboro pneumatic instrument (over 40 years old). This instrument is typically reliable and is routinely calibrated. Broken polyethylene lines supplying the pneumatic signals to the transmitters and instrument can present some problems, but in recent years, UV-resistant polyethylene has decreased potential breaking of lines due to age and exposure to UV light. Plugged lines can be a problem if the plug cannot be removed easily.

c. T-66 sparger-An electric switch activates a solenoid that opens and allows air to flow into a deep leg in the tank for solution mixing in the tank. The air flow into the tank is regulated by an orifice plate in the sparge line. The orifice plate can plug requiring the suspension of sparging operations in T66. The orifice plate would have to be removed and cleaned. This typically only requires no more than $4 \mathrm{~h}$. Plugs in the sparge line downstream of the orifice or flow meter can occasionally occur, and this can sometimes require as many as $12-24 \mathrm{~h}$ to clear. The sparger solenoid has failed, even though failure is not frequent. Rebuilds and replacements are readily available and cause very minimal down time for repairs. If necessary an alternate method of sparging T-66 is by using the in-cell air and applying air pressure down a deep leg in the tank.

d. T-66 processing lines-These are the lines that are used to transfer process solutions into and out of T-66. Typically, these lines are not an issue, though at times some lines are preferred over others, and plugged lines can be a problem if the plug cannot be removed easily or an alternate line cannot be used.

e. T-66 sampler-A $1 \mathrm{~L}$ glass bottle with a 2-line rubber stopper for sampling in Cubicle 6 is connected to a 1-line polyethylene block installed in the T-66 needle block position (SA-T66) on BR-6 using polyethylene tubing. Glass bottles, stoppers, polyethylene tubing with female ball joints, polyethylene tubing, and plastic compression fittings are kept in stock and typically readily available. (The designed sample technique is no longer employed because there are no needle blocks available to use.)

\subsubsection{Miscellaneous Solution Collection Tank, Tank T-79}

T-79 has several systems that are required to operate properly to maintain functionality. Typically, tank pit tanks like T-79 do not have many physical or mechanical issues that interrupt routine operations. Plugging instrument probes or broken instrument lines can pose some minor problems, but these problems are a low probability. 
a. Tank T-79-A nominal 68.9 L tank constructed of HC with Zr probes located in Tank Pit 7 and is typically used to collect miscellaneous solutions as needed.

b. T-79 liquid level, density, and pressure are read out and recorded on an original Foxboro pneumatic instrument (over 40 years old). This instrument is typically reliable and is routinely calibrated. Broken polyethylene lines supplying the pneumatic signals to the transmitters and instrument can present some problems, but in recent years, UV-resistant polyethylene has decreased potential breaking of lines due to age and exposure to UV light. Plugged lines can be a problem if the plug cannot be removed easily.

c. T-79 sparger-An electric switch activates a solenoid that opens and allows air to flow into a deep leg in the tank for solution mixing in the tank. The air flow into the tank is regulated by an orifice plate in the sparge line. The orifice plate can plug requiring the suspension of sparging operations in $\mathrm{T}$ 79. The orifice plate would have to be removed and cleaned. This typically only requires no more than $4 \mathrm{~h}$. Plugs in the sparge line downstream of the orifice or flow meter can occasionally occur, and this can sometimes require as many as $12-24 \mathrm{~h}$ to clear. The sparger solenoid has failed, even though failure is not frequent. Rebuilds and replacements are readily available and cause very minimal down time for repairs. If necessary an alternate method of sparging T-79 is by using the in-cell air and applying air pressure down a deep leg in the tank.

d. T-79 processing lines-These are the lines that are used to transfer process solutions into and out of T-79. Typically, these lines are not an issue, though at times some lines are preferred over others, and plugged lines can be a problem if the plug cannot be removed easily or an alternate line cannot be used.

e. T-79 sampler-A $1 \mathrm{~L}$ glass bottle with a 2-line rubber stopper for sampling in Cubicle 7 is connected to a 1-line polyethylene block installed in the T-79 needle block position (SA-T79) on BR-7 using polyethylene tubing. Glass bottles, stoppers, polyethylene tubing with female ball joints, polyethylene tubing, and plastic compression fittings are kept in stock and typically readily available. (The designed sample technique is no longer employed because there are no needle blocks available to use.)

\subsection{Miscellaneous CLEANEX Operations}

As miscellaneous solutions containing significant recoverable amounts of material are collected during a typical HFIR target rod processing campaign, the solutions are processed through a CLEANEX extraction operation. An organic extraction stage is added to T-23 (sometimes T-21 can be used instead of T-23) and the solutions are transferred into T-23 from their perspective collection tanks and the CLEANEX extraction is performed. Many extractions can be performed in T-23 until the organic is loaded with material or the organic extractant has been in use for a significant amount of time and begins to degrade. At this point the CLEANEX extractant is scrubbed and stripped of the product material and the organic disposed of as waste. 


\subsection{Chemical Make Ups}

11.1.1 M-1 and M-2-One or both are typically used for the make up of the CLEANEX acid strip solutions $\left(\mathrm{HCl}\right.$ or $\mathrm{HNO}_{3}$ ). Refer to Section 1.2 for a discussion of these two tanks and their associated lines and valves.

11.1.2 M-3-Typically used for the make up of the CLEANEX extractant solution, which consists of $1 M$ HDEHP [di-(2-ethylhexyl) phosphoric acid] in normal paraffin hydrocarbon (NPH). Refer to Section 2.1.2 for a discussion of M-3 and its associated lines and valves.

11.1.3 M-703 - A vacuum/pressure transfer tank used for transferring organic solutions from drums containing organic chemicals located in an adjacent room to the MUA. Refer to Section 2.1.3 for a discussion of M-703 and its associated lines and valves.

11.1.4 M-70 - A pressure transfer tank used for transferring aqueous chemicals to tanks in the tank pit and cubicles. Refer to Section 2.1.4 for a discussion of M-70 and its associated lines and valves.

11.1.5 M-71 - A pressure transfer tank used for transferring aqueous and organic chemicals to tanks in the tank pit and cubicles. Refer to Section 2.1.5 for discussion of M-71 and its associated lines and valve.

11.2 Miscellaneous CLEANEX extraction, scrubbing, stripping, and organic cleanout operations.

11.2.1 M-704 and M-706 are MUA tanks (used for the addition of $\mathrm{HCl}$ and $\mathrm{NaOCl}$ ). Refer to Section 1.4.2 for discussion of M-704. Refer to Section 2.2.3 for discussion of M-706.

\subsubsection{Miscellaneous CLEANEX Extraction}

a. For the initial extraction, CLEANEX extractant ( $1 M$ HDEHP in NPH) is added to T-23 from M-71. Refer to Sections 2.3.4 and 2.1.5 for discussion of T-23 and M-71, respectively. Miscellaneous solution collections from flushing, material recovery operations and rework solutions are transferred into T-23 in batches to extract the product materials into the organic stage in $\mathrm{T}-23$. The acid concentration of the solution is adjusted to a low acid concentration using additions of $\mathrm{NaOH}$ solution. At the low acid, the actinide product extracts into the organic from the aqueous solution. The $\mathrm{NaOH}$ additions are typically made from M-704 or M-706. These two tanks are discussed in Sections 1.4.2 and 2.2.3.

b. Samples are pulled from T-23 during the extraction to confirm that the extraction is complete. T-23 sampling is discussed in Section 2.3.4.e.

\subsubsection{Miscellaneous CLEANEX Extraction Raffinate Transfer}


Once the extraction is confirmed complete, the extraction raffinate is transferred (phase separated) from T-23 to a collection tank (usually T-21 or T-20). The transfer route is from T-23 to T-21 using T-607 vacuum/pressure tank on BR-6 to perform the transfer.

Cell equipment utilized:

a. T2302 GN in Cubicle 4 right DC well-Standard Zr GN outlet from T-23

(Section 2.3.2.I) with polyethylene line connected to C4C601 GN, fabricated in house. Typically spares are available

b. C4C601 GN on BR-4-Standard Zr GN with polyethylene line connected to T2302 GN in Cubicle 4 right DC well. Typically, spares are readily available. Previously discussed in Section 2.3.2.k.

c. C4C601 jumper-Hard piped jumper located in Cubicle 4, from Cubicle 4 left DC well to C4C601 GN on BR-4. Previously discussed in Section 2.3.2.j.

d. C4C601 jumper-Hard piped jumper between Cubicle 6 left DC well and Cubicle 4 left DC well. Previously discussed in Section 2.3.2.i.

e. C4C601 GN in Cubicle 6 left DC well-Standard Zr GN located in Cubicle 6 left DC well from hard piped jumper between Cubicle 6 DC well and Cubicle 4 DC well with polyethylene line connected to T60702 GN. Typically, spares are readily available. Previously discussed in Section 2.3.2.h.

f. T60702 GN on BR-6-Standard Zr GN located on BR-6 (Previously discussed in Section 9.2.8.b.) with polyethylene line connected to C4C601 GN. (Fabricated in house. Typically spares are available.)

g. Phase Separator PS-607 on BR-6-Previously discussed in Section 9.2.8.c.

h. T-607 on BR-6-Previously discussed in Section 9.2.8.d.

i. HV-T607on BR-6-Previously discussed in Section 9.2.8.e.

j. T60703 GN on BR-6-Previously discussed in Section 9.2.8.f. with polyethylene line connected to T2103 GN.

k. T2103 GN in Cubicle 6 right DC well with polyethylene line connected to T60703 GN, fabricated in house. Typically spares are available.

l. C7403 GN in Cubicle 7 left DC well-Plugged (teed to T2103 line )

m. KP-607 on BR-6-Previously discussed in Section 9.2.8.i.

n. CU6AJ-In-cell air jet used for vacuum source. Typically spares are available.

o. Polyethylene tubing - For vacuum lines, pressure lines, demineralized water lines, and portions of the transfer route. Male ball joints and plastic compression fittings are readily available.

\subsubsection{Miscellaneous CLEANEX Scrubbing}

a. Two $0.03 \mathrm{M}$ acid scrubs are used. The first scrub is added to T-23 from M70. M-70 is discussed in Section 2.1.4. 
b. Phase modifier (0.2 M basic Alamine 336 in Exxon Aromatic 150) is made up utilizing the M-703 vacuum/pressure transfer system Refer to Section 2.1.3 for discussion of this system. The $0.2 \mathrm{M}$ Alamine 336 is made up in the MUA the fume hood and contacted with $6 \mathrm{M} \mathrm{HCl}$ to acidify the phase modifier. The phase modifier is then added to T-60 from M-71 to retain the Fe constituent during stripping operations. Refer to Section 2.1.5 for discussion of M-71.

c. The scrub is sparged, settled, and then sampled in T-23 to confirm that the scrub meets acidity specifications and to determine activity levels in the aqueous scrub solution. T-23 sampling is discussed in Section 2.3.4.e.

d. Once the scrub is confirmed to meet specifications, it is transferred (phase separated) from T-23 to a collection tank (usually T-21 or T-20). The transfer route is from T-23 to T-21 using T-607 vacuum/pressure tank on BR-6. The transfer route is discussed in Section 11.2.3.

e. At this point, the organic in T-23 is transferred to T-60 using the T-607 vacuum/pressure transfer system discussed in Section 11.2.3, but the T-607 discharge (T60702 GN) polyethylene line is connected to T6007 GN (located in Cubicle 6 left DC well) to go to T-60 instead of T-21. The T6007 GN is a standard Ta GN fabricated in house, and typically spares are available.

f. The second scrub is added to T-23, sparged, and transferred on to T-60 as outlined in Section 11.2.3 and in Section 11.2.4.d to act as a flush.

g. The second scrub is sparged, settled, and then sampled in T-60 to confirm that the scrub meets acidity specifications and to determine activity levels in the aqueous scrub solution. T-60 sampling is discussed in Section 6.2.1.e.

h. Once the scrub is confirmed to meet specifications, it is transferred (phase separated) from T-60 to a collection tank (usually T-21 or T-20). Section 11.2.5 below details the transfer route for the second scrub.

\subsubsection{Miscellaneous CLEANEX Second Scrub Transfer}

Once the scrub is confirmed to meet specifications, it is transferred (phase separated) from T-60 to T-21. The transfer route is from T-60 to T-21 using T-734 vacuum/pressure tank on RR-7 to perform the transfer.

Cell equipment utilized:

a. T6002 GN in Cubicle 6 right DC well—Standard Ta GN outlet from T-60 with polyethylene line connected to T73402 GN on RR-7 in Cubicle 7; fabricated in house. Typically spares are available.

b. T73402 GN on RR-7-Standard Ta GN (Section 1.6.1.d) with polyethylene line connected to T6002 GN.

c. PS-733on RR-7-Refer to Section 1.6.1.e for discussion of PS-733.

d. HV-T734-2 position on RR-7-Refer to Section 1.6.1.f for discussion of HV-T734-2.

e. T-734 on RR-7-Refer to Section 1.6.1.g for discussion of T-734. 
f. HV-T734-3 position on RR-7-Refer to Section 1.6.1.h for discussion of HV-T734-3.

g. HV-T734-1 position on R-Refer to Section 1.6.1.k for discussion of HVT734-1.

h. T73404 GN on RR-7-Standard Ta GN outlet from T-734 (Previously discussed in Section 1.6.1.i.) with polyethylene line connected to C7403 GN to T-21. Fabricated in house. Typically spares are available.

i. C7403 GN in Cubicle 7 left DC well-Standard Zr GN inlet into T-21 with polyethylene line connected to T73404 GN on RR-7 in Cubicle 7 (tees into T2103 line from Cubicle 6 right DC well) (Previously discussed in Section 2.3.2.m.); fabricated in house. Typically spares are available.

j. $\quad$ T2103 GN in Cubicle 6 right DC well—Standard Zr GN inlet into T-21 with polyethylene plug installed (tees into C7403 line from Cubicle 7 left DC well) (Previously discussed in Section 11.2.3.k.); fabricated in house. Typically spares are available.

k. KP-735 on RR-7-Refer to Section 1.6.1.l for discussion of KP-735.

l. CU7AJ-In-cell air jet used for vacuum source. Previously discussed in Section 1.4.1.p.

m. Polyethylene tubing-For vacuum lines, pressure lines, demineralized water lines, and portions of the transfer route. Male ball joints and plastic compression fittings are readily available. Previously discussed in Section

\subsection{1.q.}

\subsubsection{Miscellaneous CLEANEX Stripping}

NOTE: Eight strips of the pregnant organic in T-60 are programed. Additional strips may be added to meet desired activity goals for stripping.

a. Once scrubbing is complete, phase modifier ( $0.2 M$ basic Alamine 336 in Exxon Aromatic 150) is made up per Section 11.2.4.b and added to T-60 from Tank M-71 in the MUA.

b. Each strip solution is added using the same equipment and route as the scrubs in Sections 2.1.4 and 11.2.4.a.

c. Each strip is sparged and settled in T-60, and the eighth strip solution is sampled for activities. T-60 sampling is discussed in Section 6.2.1.e.

d. Once the strip sample results meet the desired activity limits, the organic phase in T-60 is sampled. T-60 sampling is discussed in Section 6.2.1.e.

11.2.7 Miscellaneous CLEANEX Strip Transfer (T-60 to T-604)

Each strip is transferred (phase separated) from T-60 to T-604 and then from T604 to a strip collection tank, T-40, to prevent the transfer of any entrained "pregnant organic" to the strip collection tank. The transfer route from T-60 to T604 utilizes T-734 vacuum/pressure tank on RR-7 to perform the transfer.

Cell equipment utilized: 
a. T6002 GN in Cubicle 6 right DC well—Refer to Section 11.2.5.a for discussion of T6002 GN

b. T73402 GN on RR-7-Standard Ta GN (Previously discussed in Section 1.6.1.d.) with polyethylene line connected to T6002 GN

c. PS-733 on RR-7-Refer to Section 1.6.1.e for discussion of PS-733.

d. HV-T734-2 position on RR-7-Refer to Section 1.6.1.f for discussion of HV-T734-2.

e. T-734 on RR-7-Refer to Section 1.6.1.g for discussion of T-734.

f. HV-T734-3 position on RR-7-Refer to Section 1.6.1.h for discussion of HV-T734-3.

g. T73404 GN on RR-7-Standard Ta GN outlet from T-734 with polyethylene line connected to C7403 GN to T-21. Refer to Section 1.6.1.i for discussion of T73404 GN.

h. C7405 GN in Cubicle 7 left DC well—Standard Zr GN inlet into T-604 (Previously discussed in Section 2.3.7.h.) with polyethylene line connected to T73404 GN on RR-7 in Cubicle 7; fabricated in house. Typically spares are available

i. HV-T734-1 position on RR-7-Refer to Section 1.6.1.k for discussion of HV-T734-1.

j. $\quad$ KP-735 on R-7-Refer to Section 1.6.1.l for discussion of KP-735.

k. CU7AJ-In-cell air jet used for vacuum source. Previously discussed in Section 1.4.1.p.

l. Polyethylene tubing - For vacuum lines, pressure lines, demineralized water lines, and portions of the transfer route. Male ball joints and plastic compression fittings are readily available. Previously discussed in Section

1.4.1.q.

11.2.8 Miscellaneous CLEANEX Strip Intermediate Receiving Tank, Evaporator T-604 Refer to Sections 2.3.8 and 2.3.9 for discussion of evaporator T-604.

\subsubsection{Miscellaneous CLEANEX Strip Transfer (T-604 to T-40)}

Once the strip solution is received into T-604, each strip is sparged and settled in T-604. Each strip is transferred (phase separated) from T-604 to a strip collection tank, T-40. The transfer route from T-604 to T-40 utilizes P-653 vacuum/pressure diaphragm pump on BR-6 to perform the transfer.

Cell equipment utilized:

a. 7PDV-604-1 (Type D set to 7) on BR-6-Previously discussed in Section 2.3.10.a.

b. PS-604 on BR-6-Previously discussed in Section 2.3.10.b.

c. 2-line block at HCV-P604-1 position on BR-6-With PVC valve and polyethylene line connected to the right side; fabricated in house. Typically spares are available. Previously discussed in Section 2.3.10.c.

d. P65301 GN on BR-6-Previously discussed in Section 2.3.10.d. 
e. P-653 on BR-6-Previously discussed in Section 2.3.10.e.

f. P65302 GN on BR-6-Previously discussed in Section 2.3.10.f.

g. Special FDV at FDV-T604 position on BR-6 (set with GN at top )-Used with polyethylene line to C4C601 GN. Refer to Section 2.3.10.g for discussion of FDV-T604.

h. C4C601 GN in Cubicle 6 left DC well-With polyethylene line from special FDV at FDV-T604 to hard piped jumper between Cubicle 6 DC well and Cubicle 4 DC well, standard Zr GN. Previously discussed in Section 3.6.2.h.

i. C4C601 jumper-Hard piped jumper between Cubicle 6 left DC well and Cubicle 4 left DC well. Previously described in Section 3.6.2.i above.

j. C4C601 jumper-Hard piped jumper located in Cubicle 4, from Cubicle 4 left DC well to C4C601 GN on BR-4. Previously discussed in Section 3.6.2.j.

k. C4C601 GN on BR-4-Standard Zr GN with polyethylene line connected to T4003 GN in Cubicle 4 left DC well. Previously discussed in Section 3.6.2.k.

l. T4003 GN in Cubicle 4 left DC well (teed with T4013 line in Cubicle 6 left DC well)—Standard Zr GN. Typically, spares are readily available. Previously discussed in Section 3.6.2.k.

m. T4013 in Cubicle 6 left DC well (teed to T4003 line in Cubicle 4 left DC well) - Plugged with a male plug at the DC position in the well. Previously discussed in Section 3.6.2.I.

n. 7PDV-604-2 (Type D set to 2) on BR-6-Previously discussed in Section 2.3.10.h.

o. 2-line block at HCV-P604-2 position on BR-6-Previously discussed in Section 2.3.10.i.

p. Polyethylene tubing-For vacuum lines, pressure lines, demineralized water lines, and portions of the transfer route. Male ball joints and plastic compression fittings are readily available. Previously discussed in Section

\subsection{1.q.}

\subsubsection{Miscellaneous CLEANEX Confirming Strip}

a. Once the stripping of T-60 is confirmed complete from the T-60 strip sample analysis, a confirming strip (flush) is added to T-604 from M-70 or equivalent tank as the scrubs and regular strip additions as discussed in Sections 2.1.4 and 11.2.4.a.

b. The confirming flush is sparged, settled, and sampled in T-604. T-604 sampling is discussed in Section 2.3.8.e.

c. The confirming flush is transferred from T-604 to T-40 as described in Section 11.2.9.

d. Once the confirming flush sample analysis is determined to meet the activity specification to terminate stripping, the organic phase in T-604 is sampled. $\mathrm{T}-604$ sampling is discussed in Section 2.3.8.e. 
e. T-40 strip product evaporations are routinely performed during the stripping operations. Refer to Sections 9.2.11 and 9.2.12 above for a discussion of the Tank T-40 evaporator.

f. The CLEANEX strip product in T-40 is routinely sampled after the final confirming flush has been received from T-604. Refer to Section 9.2.11.e above for a discussion of the tank T-40 sampling.

\subsubsection{Miscellaneous CLEANEX Organic Cleanout}

Once stripping operations have been confirmed complete based on T-60 and T604 sample results, organic cleanouts of these tanks may be performed.

a. Addition of a specified volume of low concentration $(<5 M) \mathrm{NaOH}$, is made to T-60 and to T-604 to neutralize the acidic aqueous heel and acidic organic for transfer to F-126 waste tank via routes already discussed.

b. The tanks are sparged and sampled to confirm that the solutions are neutralized or slightly basic. Sampling of T-60 and T-604 is discussed in Section 6.2.1.e and Section 2.3.8.e, respectively.

c. T-60 and T-604 contents are transferred to F-126 utilizing T-734 vacuum/pressure tank on RR-7 to perform the transfer. The transfer routes are discussed below in Sections 11.2.12 and 11.2.13.

d. Both T-60 and T-604 are flushed to F-126 with diluent and water using the T-734 vacuum/pressure tank on RR-7 to perform the transfer. The diluent is obtained for flushing by utilizing the M-703 vacuum/pressure transfer system previously discussed in Section 2.1.3.

e. At times when the waste jets from T-60 and T-604 to F-115 waste tank are operating, water flushes are added to T-60 and T-604, sparged, and jetted to F-115. Otherwise the flushes are transferred to F-126 via the routes outlined in Section 11.2.12 and 11.2.13. If there is a steam outage, then the steam jets cannot be utilized for transfers. Steam jets can fail, and because they are located in the hot cell tank pits, it is very difficult to replace them, even with the availability of spare jets. At times, the jets can get plugged with debris, and it can require significant effort to unplug it causing operational delays. This is why the route to F-126 waste tank is often utilized.

f. Any remaining diluent in T-60 and T-604 is steam distilled by adding water and evaporating off a specified amount of water to T-34, the evaporator condensate collection tank. T-60 evaporator operation is discussed in Sections 6.2.1 and 6.2.2, and T-604 evaporator operation is discussed in Sections 2.3.8 and 2.3.9.

g. The remainder of the water in the tanks after the steam distillation is complete is jetted to F-115 or transferred to F-126 per previously discussed routes. Refer to Section 9.2.15.e for discussion of T-60 and T-604 steam jets.

11.2.12 T-60 Miscellaneous CLEANEX Waste Organic Transfer 
Once T-60 has been confirmed to be basic (or neutral) based on T-60 sample results, transfer of the waste organic may be performed.

NOTE: Typically, the portion of the transfer route from T-60 to T-734 will not be changed, but it is discussed again below in Sections 11.2.14.a-i. Only the discharge line from $T-734$ will be required to be rerouted to the appropriate collection tanks.

Cell equipment utilized:

a. T6002 GN in Cubicle 6 right DC well—Standard Ta GN outlet from T-60 with polyethylene line connected to T73402 GN on RR-7 in Cubicle 7. Refer to Section 11.2.5.a for discussion of T6002 GN.

b. T73402 GN on RR-7-Standard Ta GN (Section 1.6.1.d) with polyethylene line connected to T6002 GN.

c. $\quad$ PS-733 on RR-7-Refer to Section 1.6.1.e for discussion of PS-733.

d. HV-T734-2 position on RR-7-Refer to Section 1.6.1.f for discussion of HV-T734-2.

e. T-734 on RR-7-Refer to Section 1.6.1.g for discussion of T-734.

f. HV-T734-3 position on RR-7-Refer to Section 1.6.1.h for discussion of HV-T734-3.

g. HV-T734-1 position on RR-7-Refer to Section 1.6.1.k for discussion of HV-T734-1.

h. T73404 GN on RR-7-Standard Ta GN (Previously discussed in Section 1.6.1.i.) with polyethylene line connected to 1-line block at SA-F126 position.

i. 1-line polyethylene block at SA-F126 needle block position on BR-7-Refer to Section 9.2.16.i for discussion of 1-line block at SA-F126 position.

j. $\quad$ KP-735 on RR-7-Refer to Section 1.6.1.I for discussion of KP-735.

k. CU7AJ-in-cell air jet used for vacuum source. Previously discussed in Section 1.4.1.p.

l. Polyethylene tubing-For vacuum lines, pressure lines, demineralized water lines, and portions of the transfer route. Male ball joints and plastic compression fittings are readily available.

\subsubsection{T-604 Miscellaneous CLEANEX Waste Organic Transfer}

Once T-604 has been confirmed to be basic (or neutral) based on T-604 sample results, transfer of the waste organic may be performed.

NOTE: Typically, the portion of the transfer route from T-604 to T-734 is the only thing required to be set because the T-734 system was previously used to dispose of organic from T-60, but it is discussed again below in Sections 11.2.15.a-i.

Cell equipment utilized: 
a. 7PDV-604-1 (Type D set to 7) on BR-6-Previously discussed in Section 2.3.10.a.

b. Special 2-line block at PS-604 position on BR-6-With polyethylene tubing connected to the left side Previously discussed in Section 2.3.17.b.

c. T73402 GN on RR-7-Standard Ta GN (Previously discussed in Section 1.6.1.d.) with polyethylene line connected to the left side of the 2-line block at PS-604.

d. PS-733on RR-7-Refer to Section 1.6.1.e for discussion of PS-733.

e. HV-T734-2 position on RR-7-Refer to Section 1.6.1.f for discussion of HV-T734-2.

f. $\quad$ T-734 on RR-7-Refer to Section 1.6.1.g for discussion of T-734.

g. HV-T734-3 position on RR-7-Refer to Section 1.6.1.h for discussion of HV-T734-3.

h. HV-T734-1 position on RR-7-Refer to Section 1.6.1.k for discussion of HV-T734-1.

i. T73404 GN on RR-7-Polyethylene line connected from T73404 GN (Previously discussed in Section 1.6.1.i) to 1-line block at SA-F126 position.

j. 1-line polyethylene block at SA-F126 needle block position on BR-6-Used with a polyethylene line connected from T73404 GN. Previously discussed in Section 9.2.16.i.

k. KP-735 on RR-7-Refer to Section 1.6.1.I for discussion of KP-735.

l. CU7AJ-In-cell air jet used for vacuum source. Previously discussed in Section 1.4.1.p.

m. Polyethylene tubing-For vacuum lines, pressure lines, demineralized water lines, and portions of the transfer route. Male ball joints and plastic compression fittings are readily available. Previously discussed in Section

\subsection{1.q.}

\subsection{BERKEX Operations}

The BERKEX operations are performed utilizing equipment located on RR-4 in Cubicle 4. The feed solution is composited from selected ${ }^{249} \mathrm{Bk}$ cuts from the cation exchange operations previously performed in Cubicle 4. This feed solution is loaded on to the resin in the C-402 Loading Column. The column is flushed with a small amount of demineralized water. The Bk product is stripped off with $2 \times 200 \mathrm{ml}$ of $8 \mathrm{MHNO}_{3}$ into a $1 \mathrm{~L}$ polyethylene bottle to reduce the volume and adjust the acid of the feed solution. The feed is allowed to "age" overnight because better extraction, scrubbing, and stripping of the Bk have been observed. The Bk feed solution is extracted into $500 \mathrm{ml}$ of $0.5 \mathrm{M} \mathrm{HDEHP}$ in dodecane (prepared by washing with $6.8 \mathrm{M} \mathrm{HNO}_{3}$ and $0.32 \mathrm{M} \mathrm{NaBrO}_{3}$ ), scrubbed ( $2 \mathrm{M} \mathrm{NaBrO}_{3}$ ), and stripped (strip solution is $30 \mathrm{ml}$ of $30 \% \mathrm{H}_{2} \mathrm{O}_{2}$ and $250 \mathrm{ml}$ and $8 \mathrm{M} \mathrm{HNO}_{3}$ ) to the final product bottle. Once the Bk product is collected, the extraction equipment is flushed to T-45. The raffinate, scrubs, product, and wash solutions are sampled at the end. 


\subsection{Chemical Make Ups}

12.1.1 Eight $1 \mathrm{~L}$ polyethylene bottles and six $250 \mathrm{ml}$ polyethylene bottles are labeled for use during the BERKEX operations. An adequate inventory of $1 \mathrm{~L}$ polyethylene bottles is typically maintained.

12.1.2 $1,000 \mathrm{ml}$ of $2.0 \mathrm{M} \mathrm{NaBrO}_{3}$ is made up and used for washing the organic extractant before use and as a scrub solution during the scrubbing operations. It is poured up in appropriately labeled polyethylene bottles. An adequate inventory of $\mathrm{NaBrO}_{3}$ is typically maintained for process operation requirements.

12.1.3 5.5 $\mathrm{L}$ of $8 \mathrm{M} \mathrm{HNO}_{3}$ is made up and used for washing the organic extractant before use and as the strip solution during the stripping operations. It is poured up in appropriately labeled polyethylene bottles. An adequate inventory of concentrated $\mathrm{HNO}_{3}$ is typically maintained for chemical make ups for process operation requirements.

12.1.4 $600 \mathrm{ml}$ of $0.5 \mathrm{M}$ HDEHP in dodecane is made up and washed with $6.8 \mathrm{M} \mathrm{HNO}_{3}$ and $0.32 \mathrm{M} \mathrm{NaBrO}_{3}$ before use. It is poured up in an appropriately labeled polyethylene bottle. An adequate inventory of HDEHP and dodecane is typically maintained for process operation requirements.

12.1.5 Two $30 \mathrm{ml}$ volumes of $30 \% \mathrm{H}_{2} \mathrm{O}_{2}$ are measured out and poured up in appropriately labeled polyethylene bottles for use in the stripping operations. An adequate inventory of $30 \% \mathrm{H}_{2} \mathrm{O}_{2}$ is typically maintained for process operation requirements.

12.1.6 Sample thief pipettes-A small glass sample thief (small volume $\sim 5 \mathrm{ml}$ pipette) and small rubber bulb is used for sampling during the BERKEX operations, typically sample thief pipettes and rubber bulbs, are readily available. As warranted, small plastic pipettes are used for sampling as well. These pipettes are readily available, and an ample inventory is usually on hand.

\subsection{BERKEX Feed Adjustment}

\subsubsection{Preparations}

a. Several polyethylene bottles, previously used in the cation exchange operations are used as well as several new polyethylene bottles labeled for these BEKEX operations.

b. Alpha monitoring instrumentation—Previously described in Section 3.4.9.a above.

c. Neutron monitoring instrumentation-Previously described in Section 3.4.9.b above.

d. Alpha and neutron monitoring instrumentation support equipment and materials-Previously described in Section 3.4.9.c and 3.4.9.d above. 
12.2.2 Pre-run PVC-T404 Diaphragm Test

NOTE: All valves, polyethylene lines, polyethylene bottles, and alpha probes are typically maintained in adequate supply to support these operations.

Cell equipment utilized:

a. V-13 and V-14-Hoke ${ }^{\circledR}$ ball valves that are procured and then modified in house. Typically spares are available. Previously discussed in Section 4.2.3.i above.

b. V-T408-1, V-T408-2, V-T408-3, V-T404-1, V-T404-2, V-T404-3, and VT404-4-Bonnet needle valves. Previously described in Section 4.2.3.n above.

Control Room equipment utilized:

c. PIC-T404 (Previously described in Section 4.2.3.k above.)

MUA equipment utilized:

d. PCV-T404-Previously described in Section 4.2.3.I above.

e. PI-T404-2-Previously described in Section 4.2.3.m above.

f. Miscellaneous equipment and supplies such as funnels, polyethylene bottles, polyethylene tubing, bottle holders, etc. are typically available. Previously discussed in Section 4.2.1.h above.

\subsubsection{Pre-run System Water Test}

Cell equipment utilized:

a. Tank T-404-Previously described in Section 4.2.3.g above.

b. Polyethylene line $-1 / 4$ in. diameter polyethylene line with male ball joint connected to female ball joint at V-15 on RR-4, long enough to reach the cubicle floor. Typically, an ample supply of these polyethylene lines is available.

c. V-13 and V-14-Hoke ${ }^{\circledR}$ ball valves, procured and then modified in house. Typically spares are available. Previously discussed in Section 4.2.3.i above.

d. PI-T404, pressure gauge (-0-30 in. water vacuum and 0-100 psig pressure) for T-404 located on RR-4. Previously described in Section 4.2.3.h above.

e. CU4AJ-Cubicle 4 vacuum. Previously described in Section 4.2.3.j above.

f. RE-401-Previously described in Section 4.2.1.f above.

Control Room equipment utilized:

g. PIC-T404-Previously described in Section 4.2.3.k above.

MUA equipment utilized:

h. PCV-T404-Previously described in Section 4.2.3.l above.

i. PI-T404-2-Previously described in Section 4.2.3.m above.

j. V-T408-1, V-T408-2, V-T408-3, V-T404-1, V-T404-2, V-T404-3, and VT404-4-Bonnet needle valves. Previously described in Section 4.2.3.n above. 
k. Miscellaneous equipment and supplies such as funnels, polyethylene bottles, polyethylene tubing, bottle holders, etc. are typically available.

12.2.4 Load Bk onto C-402 Loading Column

a. Bk feed solution transfer from the feed bottle to T-404 through C-402 to raffinate collection bottle(s) using the route detailed in Section 12.2.3 above.

b. Raffinate collection in polyethylene bottles from Section 12.1.1 above.

c. Column flush the loaded column with water from T-404 through C-402 using the same route as detailed in Section 12.2.3 above.

d. Water flush collection in polyethylene bottles from Section 12.1.1 above.

12.2.5 Strip Bk Product off C-402 Loading Column

a. Strip solution $\left(2 \times 200 \mathrm{ml}\right.$ and $1 \times 100 \mathrm{ml}$ of $\left.8 \mathrm{MHNO}_{3}\right)$ transfers through C402 to strip the Bk off the resin in C-402 using the route detailed in Section 12.2.3 above.

b. Bk strip solutions collection in the polyethylene bottle for Bk feed from Section 12.1.1 above.

c. Column flush with water from T-404 through C-402 using the same route as detailed in Section 12.2.3 above.

d. Collect the flush water in a specified polyethylene bottle for flush solution from Section 12.1.1 above.

12.2.6 Acidify, Sparge, and Sample the Adjusted Bk Feed

a. $100 \mathrm{ml}$ of concentrated $\mathrm{HNO}_{3},-$ An adequate inventory of concentrated $\mathrm{HNO}_{3}$ is typically maintained for use in process operation requirements.

b. Sample of the acidified/adjusted Bk feed using a clean thief pipette (from Section 12.1.6 above).

\subsection{BERKEX System Setup}

\subsubsection{Preparations}

a. Pinch clamp (V-25)_Located on RR-4. Flexible tubing with a pinch clamp is typically used in routine laboratory work. The clamp has not been replaced but has needed lubricating from time to time. A suitable replacement should be easily obtained in case of a failure.

b. Tank T-411 (Bk extraction pot) located on RR-4-A $4.5 \mathrm{~L}$ glass tank with a flanged top, integrated shallow line, and a drain off the bottom of the tank. This tank has been in service for $>35$ years. The flange gasket is the most common failure of flanged glass tanks. Removal of this tank from the rack has never been attempted, but removal of similar tanks has been performed successfully in the past. The glass should be replaced if the tank is ever removed from the rack. There are currently no spare flanges. If the flange 
fails, it may take up to a week to have a flange fabricated and another week to install it.

c. $1 / 2$ in. JACO fitting with a section of $1 / 2$ in. polyethylene line with a female ball joint that attaches to the T-411 drain line-Spare $1 / 2$ in. JACOs with a female ball joint attached are also needed. Typically, spares are readily available.

d. Phase separator-Connected to T-411 drain line that is made by connecting a section of Tygon tubing to the top of the pipette. Typically, spares are readily available.

e. Funnel with adapter-For installation on T4503 GN in Cubicle 4 right DC. Typically, spares are readily available.

f. Tank T-409 (vacuum KOP) located on RR-4-A 4.5 L glass tank with a flanged top, integrated shallow line, and a drain off the bottom of the tank. There are currently no spare flanges. If the flange fails, it may take up to a week to have a flange fabricated and another week to install it. Typical failure would be the flange gasket.

g. Specially prepared 3/8 in. polyethylene tubing with a male ball joint on one end and a female ball joint on the other end-Used as an extension from the vacuum connection for T-409. Typically, spares are readily available.

h. Specially prepared 3/8 in. polyethylene tubing with male ball joints on both ends-Used to connect T-409 dip line to T-411 vacuum connection. Typically, spares are readily available.

i. Calibrated and labeled 1 L polyethylene bottle labeled "SR-1" — Typically kept in stock in ample supply and readily available. This bottle is described in Section 12.1.1.a above.

j. $\quad 250 \mathrm{ml}$ of $2.0 \mathrm{M} \mathrm{NaBrO}_{3}$ (made up per Section 12.1.2 above) in a calibrated $250 \mathrm{ml}$ polyethylene bottle (bottle from per Section 12.1.1.a above)— Typically kept in stock in ample supply and readily available.

k. Concentrated $\mathrm{HNO}_{3}$-Approximately $1 \mathrm{~L}$ in a calibrated and labeled polyethylene bottle (Refer to Section 12.1.1.a above.). $\mathrm{HNO}_{3}$ is typically readily available in ample supply.

l. Calibrated $250 \mathrm{ml}$ polyethylene bottle-Used for measuring. Typically kept in ample supply and readily available. Sometimes an unused "cut bottle" from the cation exchange operations is used for this.

m. $600 \mathrm{ml}$ of hot feed-In a $1 \mathrm{~L}$ polyethylene bottle labeled “FEED/RAFFINATE.” Refer to Section 12.2.4 above.

n. $500 \mathrm{ml}$ of $0.5 \mathrm{M}$ HDEHP in dodecane in a $1 \mathrm{~L}$ calibrated polyethylene bottle-Refer to Sections 12.1.4 and 12.1.1.a above.

o. $1,000 \mathrm{ml}$ of $8 \mathrm{M} \mathrm{HNO}_{3}$ - Refer to Section 12.1.3 above.

p. Specially sleeved $1 \mathrm{~L}$ calibrated polyethylene bottle labeled "PRODUCT"$1 \mathrm{~L}$ polyethylene bottles and sleeving are typically readily available.

q. $3 \times 1,000 \mathrm{ml}$ quantities of $8 \mathrm{MHNO}_{3}$ in $1 \mathrm{~L}$ calibrated polyethylene bottles. Refer to Section 12.1.3 above.

r. $\quad 3 \times 250 \mathrm{ml}$ quantities of $2.0 \mathrm{M} \mathrm{NaBrO}_{3}$-Refer to Section 12.1.2 above. 
s. $2 \times 250 \mathrm{ml}$ quantities of $30 \% \mathrm{H}_{2} \mathrm{O}_{2}$ - Refer to Section $\mathbf{1 2 . 1 . 5}$ above.

\subsubsection{Equipment Setup and Testing}

a. Pinch clamp (V-25)—On the Bk extraction pot, T-411 discharge line. Refer to Section 12.3.1.a above.

b. Tank T-411 on RR-4-Refer to Section 12.3.1.b above for a discussion of T411.

c. Cubicle 4 vacuum (CU4AJ)—Refer to Section 4.2.3.j above.

d. Funnel with adapter on T4503 GN-Refer to Section 12.3.1.e above.

e. Tank T-409 on RR-4-Refer to Section 12.3.1.f above for a discussion of T409.

f. Specially prepared 3/8 in. polyethylene tubing-Used as an extension from the vacuum connection for T-409 KOP. Refer to Section 12.3.1.g above.

g. Specially prepared 3/8 in. polyethylene tubing-Connects the T-409 dip line to T-411 vacuum connection. Refer to Section 12.3.1.h above.

h. V-23 on RR-4-Vent line to T-411, Hoke ${ }^{\circledR}$ ball valve that was procured and then modified in house. Typically spares are available. Currently this valve has a broken handle, is in the open position, and the vent line is plugged with a male ball joint plug. This particular valve is located in a location on RR-4 and would be very difficult to replace.

i. Male ball joint plug-Installed on the line to V-23 on RR-4 (vent line on the Bk extraction pot, T-411). Fabricated in house. Typically spares are available

j. Vacuum gauge-Used for measuring in-cell vacuum. Typically spare gauges are readily available.

\subsection{BERKEX Extraction Pot Wash}

12.4.1 Wash solution transferred into T-411 per the route described in Section 12.3.2 above.
a. $2.5 \mathrm{~L}$ water
b. $1.0 \mathrm{~L}$ concentrated $\mathrm{HNO}_{3}$
c. $200 \mathrm{ml} 2.0 \mathrm{M} \mathrm{NaBrO}_{3}$

12.4.2 Wash solution sparged and drained from T-411 to T-45 per the route described in Section 12.3.2 above.

\subsection{BERKEX Extraction}

12.5.1 Feed transfer into T-411 per the route described in Section 12.3.2 above.

12.5.2 Transfer $90 \mathrm{ml} 2.0 \mathrm{M} \mathrm{NaBrO}_{3}$ and $10 \mathrm{ml}$ water flush into T-411 per the route described in Section 12.3.2 above.

12.5.3 Transfer $500 \mathrm{ml}$ of $0.5 \mathrm{M}$ HDEHP in dodecane into T-411 per the route described in Section 12.3.2 above. 
12.5.4 Mix for 30 min using vacuum on tank while the pinch clamp (V-25) on the drain line is open. Refer to Section 12.3.1.a above.

12.5.5 Settle solution by closing the pinch clamp (V-25) on the drain line. Refer to Section 12.3.1.a above.

12.5.6 Open the pinch clamp (V-25) on the drain line and drain the aqueous raffinate to the $1 \mathrm{~L}$ polyethylene bottle for collecting the raffinate. Refer to Section 12.3.2 above.

\subsection{BERKEX Scrubbing}

12.6.1 Transfer $90 \mathrm{ml} 2.0 \mathrm{M} \mathrm{NaBrO}_{3}$ into T-411 per the route described in Section 12.3.2 above.

12.6.2 Transfer $500 \mathrm{ml}$ 8.0 $\mathrm{M} \mathrm{HNO}_{3}$ into T-411 per the route described in Section 12.3.2 above.

12.6.3 Mix first scrub for 20 min using the vacuum on the tank while the pinch clamp (V-25) on the drain line is open. Refer to Section 12.5.4 above.

12.6.4 Settle solution by closing the pinch clamp (V-25) on the drain line. Refer to Section 12.5.5 above.

12.6.5 Open the pinch clamp (V-25) on the drain line and drain the aqueous scrub solution to the $1 \mathrm{~L}$ polyethylene bottle for collecting the first scrub. Refer to Section 12.3.2 above.

12.6.6 Repeat Sections 12.6.1-12.6.5 for a second scrub.

\subsection{BERKEX Stripping}

12.7.1 Transfer $30 \mathrm{ml}$ of $30 \% \mathrm{H}_{2} \mathrm{O}_{2}$ into T-411 per the route described in Section 12.3.2 above.

12.7.2 Transfer $250 \mathrm{ml} 8.0 \mathrm{M} \mathrm{HNO}_{3}$ into T-411 per the route described in Section 12.3.2 above.

12.7.3 Mix first strip for 30 min using vacuum on tank while the pinch clamp (V-25) on the drain line is open. Refer to Section 12.5.4 above.

12.7.4 Settle solution by closing the pinch clamp (V-25) on the drain line. Refer to Section 12.5.5 above.

12.7.5 Open the pinch clamp (V-25) on the drain line and drain the aqueous strip solution to the $1 \mathrm{~L}$ polyethylene bottle for collecting the Bk product. Refer to Section 12.3.2 above.

12.7.6 Repeat Sections 12.7.1-12.7.5 for a second strip. 
12.7.7 Open the pinch clamp (V-25) on the drain line and drain the waste organic solution to the $1 \mathrm{~L}$ polyethylene bottle for collecting the waste organic.

\subsection{BERKEX Solution Sampling}

12.8.1 Each scrub, raffinate, waste organic, and product collection is mixed and sampled using a small glass sample thief (small volume pipette) and small rubber bulb, and then the samples are submitted for analysis. An ample supply of sample thieves and rubber bulbs are kept on hand for use.

\subsection{BERKEX Extraction Pot (T-411) and Vacuum KOP (T-409) Flush}

12.9.1 Transfer $2.0 \mathrm{~L}$ of water, $1.5 \mathrm{~L}$ of concentrated $\mathrm{HNO}_{3}$, and enough water $(>1 \mathrm{~L})$ into T-411 per the route described in Section 12.3.2 above to fill T-411 and overflow $\sim 200 \mathrm{ml}$ of solution into T-409.

12.9.2 Sparge vigorously by leaving the valves open and leaving the vacuum on.

12.9.3 Drain the solution from T-411 to T-45 per the route described in Section 12.3.2 above. The solution from T-409 should siphon out during the draining.

12.9.4 During draining, sample the flush solution by collecting a sample from the PS. An ample supply of sample bottles is kept on hand for use.

\subsection{Other Miscellaneous Equipment}

\subsection{Additional Equipment}

There are a number of other tank pit tanks, not previously discussed, that can be utilized for various purposes in support of the processing operations. Some of these tanks act as product storage tanks until the products are processed at a later time. These tanks can provide temporary storage for recycle materials until a specific series of process operations occur, at which time the solution can be recycled back into the process. In some cases, the additional tanks can be used to replace a tank that is normally used in a particular process and has become unavailable for various reasons. This versatility helps to ensure that process operations can continue without significant delays in the overall process schedule.

13.1.1 The table below lists the additional tanks available for miscellaneous uses to support the Cf Production Program. The table includes tank location, nominal maximum volume, and construction material of the tank components and tubes (process and instrument lines).

\begin{tabular}{|l|c|c|c|c|c|c|l|}
\hline \multirow{2}{*}{ TANK ID } & \multirow{2}{*}{ LOCATION } & \multirow{2}{*}{$\begin{array}{c}\text { VOLUME } \\
\text { (liters) }\end{array}$} & \multicolumn{2}{|c|}{ CONSTRUCTION MATERIAL } & \multirow{2}{*}{ SAMPLE ACCESS } \\
\cline { 4 - 7 } & & Shell & Liner & Head & Tubes & \\
\hline T-22 $^{\mathbf{1}}$ & Tank Pit 2 & 68.7 & HC & - & - & $\mathrm{Zr}$ & $\begin{array}{l}\text { GN in Cubicle 6 right DC } \\
\text { well }\end{array}$ \\
\hline T-41 $^{\mathbf{2}}$ & Tank Pit 4 & 22.1 & HC & Ta & $\mathrm{Zr}$ & $\mathrm{Ta}$ & 1-line block at SA-T41 \\
\hline
\end{tabular}




\begin{tabular}{|c|c|c|c|c|c|c|c|}
\hline$T-42^{2}$ & Tank Pit 4 & 22.1 & $\mathrm{HC}$ & $\mathrm{Ta}$ & $\mathrm{Zr}$ & $\mathrm{Ta}$ & 1-line block at SA-T42 \\
\hline $\mathrm{T}-47^{3}$ & Tank Pit 4 & 67.3 & $\mathrm{Zr}$ & - & - & $\mathrm{Zr}$ & 1-line block at SA-T47 \\
\hline$T-48^{4}$ & Tank Pit 4 & 22.2 & $\mathrm{HC}$ & Та & $\mathrm{Zr}$ & Та & $\begin{array}{l}\text { GN in Cubicle } 4 \text { right DC } \\
\text { well }\end{array}$ \\
\hline T-67 & Tank Pit 6 & 22.6 & $\mathrm{Zr}$ & - & - & $\mathrm{Zr}$ & $\begin{array}{l}\text { GN in Cubicle } 6 \text { left DC } \\
\text { well }\end{array}$ \\
\hline T-71A ${ }^{2,5}$ & Tank Pit 7 & 64.3 & $\mathrm{Zr}$ & - & - & $\mathrm{Zr}$ & 1-line block at SA-T71 \\
\hline T-73A $A^{6,7}$ & Tank Pit 7 & 22.1 & $\mathrm{HC}$ & Ta & $\mathrm{Ta}$ & Тa & $\begin{array}{l}\text { GN in Cubicle } 7 \text { left DC } \\
\text { well }\end{array}$ \\
\hline T-300² & Tank Pit 3 & 22.0 & $\mathrm{HC}$ & $\mathrm{Ta}$ & $\mathrm{Ta}$ & $\mathrm{Ta}$ & 1-line block at SA-T300 \\
\hline T-301 ${ }^{3}$ & Tank Pit 3 & 22.4 & $\mathrm{HC}$ & Та & $\mathrm{Ta}$ & Тa & 1-line block at SA-T301 \\
\hline T-302 ${ }^{2}$ & Tank Pit 3 & 22.1 & $\mathrm{HC}$ & Та & $\mathrm{Ta}$ & Та & 1-line block at SA-T302 \\
\hline T-200 ${ }^{8}$ & Tank Pit 2 & 22.3 & $\mathrm{HC}$ & Та & $\mathrm{Ta}$ & Та & N/A \\
\hline $\mathbf{T}-32^{8}$ & Tank Pit 3 & 68.3 & $\mathrm{Zr}$ & - & - & $\mathrm{Zr}$ & N/A \\
\hline
\end{tabular}

${ }^{1} \mathrm{~T}$-22 vacuum accumulator and needle block position are OOS.

${ }^{2}$ Vacuum accumulator and needle block position are still functional.

${ }^{3}$ Has functional needle block position, but no vacuum accumulator.

${ }^{4}$ Has no vacuum accumulator or needle block position.

${ }^{5} \mathrm{~T}-71 \mathrm{~A}$ has a damaged liquid level probe.

${ }^{6} \mathrm{~T}-73 \mathrm{~A}$ is an evaporator.

${ }^{7}$ T-73A vacuum accumulator and needle block position are OOS.

${ }^{8}$ Tanks are out of service due to leaks.

13.1.2 Each tank above is equipped with the following instrumentation:

a. Foxboro pneumatic instrument-The liquid level, density, and pressure (as applicable) are read out and recorded on an original Foxboro pneumatic instrument (over 40 years old). This instrument is typically reliable and is routinely calibrated. Broken polyethylene lines supplying the pneumatic signals to the transmitters and instrument can present some problems, but in recent years, UV-resistant polyethylene has decreased potential breaking of lines due to age and exposure to UV light. Plugged lines can be a problem if the plug cannot be removed easily.

b. Tank sparger-An electric switch activates a solenoid that opens and allows air to flow into a deep leg in the tank for solution mixing in the tank. The air flow into the tank is regulated by an orifice plate in the sparge line. The orifice plate can plug requiring the suspension of sparging operations in the tank. The orifice plate would have to be removed and cleaned. This typically only requires no more than $4 \mathrm{~h}$. Plugs in the sparge line downstream of the orifice or flow meter can occasionally occur. This can sometimes require as many as $12-24 \mathrm{~h}$ to clear. The sparger solenoid has failed, even though failure is not frequent. Rebuilds and replacements are readily available and cause very minimal down time for repairs. If necessary an alternate method 
of sparging the tank is by using the in-cell air and applying air pressure down a deep leg in the tank.

c. Processing lines-These are the lines that are used to transfer process solutions into and out of the tank. Typically, these lines are not an issue, though at times some lines are preferred over others, and plugged lines can be a problem if the plug cannot be removed easily or an alternate line cannot be used.

d. Sampler-A $1 \mathrm{~L}$ glass bottle with a 2-line rubber stopper for sampling in the cubicle is connected to a 1-line polyethylene block installed in a needle block position on the sample rack (if there is a functional needle block position for the particular tank being sampled) or a metal GN located in the appropriate cubicle disconnect well using polyethylene tubing. Glass bottles, stoppers, polyethylene tubing with female ball joints, polyethylene tubing, and plastic compression fittings are kept in stock and typically readily available.

\subsection{Equipment Exclusions from Cf Production Program}

13.2.1 Tank T-10 is not included in this discussion. T-10 is located in Tank Pit 1 . Tank Pit 1 also houses storage wells for special nuclear materials and has special requirements and controls that prohibit any solutions from being transferred into $\mathrm{T}-10$ without special permission.

13.2.2 With the exception of T-54 (previously discussed in Sections 7.2.1 and 7.2.2), tanks located in Tank Pit 5 and the equipment located in Cubicle 5 are not listed because they are not currently utilized in any of the process activities associated with processing HFIR Cm target rods for the ${ }^{252} \mathrm{Cf}$ Production Program.

13.2.3 Tanks T-740, T-742, C-743 and other equipment located on Cubicle 7 left rack, are not listed since they are part of the dissolver off gas treatment system (LR-7) used for removal and retention of ${ }^{131}$ I during HFIR target rod dissolution operations. As of 2003, this equipment is no longer required to operate in support of the Cf Production Program because the ${ }^{131} \mathrm{I}$ is allowed to decay to levels below release limits. If LR-7 was ever required to operate for the removal and retention of ${ }^{131} \mathrm{I}$, it would require several weeks, if not several months, to return the rack to full operation. 


\section{APPENDIX A. EQUIPMENT EVALUATION TABLE}



APPENDIX A. EQUIPMENT EVALUATION TABLE

\subsection{Target Rod Dissolution}

\begin{tabular}{|c|c|c|c|c|c|}
\hline ITEM & LOCATION & DESCRIPTION & \begin{tabular}{|} 
REFEREN \\
CE
\end{tabular} & IMPACT & COMMENTS \\
\hline Slitter rail & CUB 7 & Used to secure target rod for target rod cooling shroud (hex can removal). & 1.1 .1 & LOW & 3 \\
\hline Cutter assembly & CUB 7 & Guided cutter for cutting hex can longitudinally for removal from target rod. & 1.1 .1 & LOW & 1,2 \\
\hline Screwdriver & CUB 7 & Modified for in-cell use. & 1.1 .2 & LOW & 1 \\
\hline Miscellaneous tools & CUB 7 & Most tools support replacement of the cutter assembly blade, all modified for in-cell use. & 1.1 .2 & LOW & 1 \\
\hline Tank M-1 & MUA & Nominal $50 \mathrm{~L}$ PVC tank used for cold chemical $\mathrm{NaNO}_{3}$ make up. & 1.2 .1 & LOW & 4 \\
\hline V-M1-1 & MUA & Hayward PVC ball valve, vent line to vessel off gas (VOG) system. & 1.2.1.a & LOW & 1,2 \\
\hline V-M1-2 & MUA & Hayward PVC ball valve, solution addition line through the funnel. & 1.2.1.b & LOW & 1,2 \\
\hline V-M1-3 & MUA & Hayward PVC ball valve, 5 psig air pressure supply line. & 1.2.1.C & LOW & 1,2 \\
\hline V-M1-4 & MUA & Hayward PVC ball valve, outlet line/drain. & 1.2.1.d & LOW & 1,2 \\
\hline V-M1-6 & MUA & Hayward PVC ball valve, solution addition line. & 1.2.1.e & LOW & 1,2 \\
\hline V-M1-7 & MUA & Hayward PVC ball valve, outlet line. & 1.2.1.f & LOW & 1,2 \\
\hline V-M1-11 & MUA & Hayward PVC ball valve, sight glass valve at bottom of sight glass. & 1.2.1.g & LOW & 1,2 \\
\hline V-M1-13 & MUA & Hayward PVC ball valve, outlet line. & 1.2.1.h & LOW & 1,2 \\
\hline Tank M-2 & MUA & Nominal $50 \mathrm{~L}$ PVC tank used for cold chemical $\mathrm{NaNO}_{3}$ make up. & 1.2 .2 & LOW & 4 \\
\hline V-M2-1 & MUA & Hayward PVC ball valve, vent line to VOG system. & 1.2.2.a & LOW & 1,2 \\
\hline V-M2-2 & MUA & Hayward PVC ball valve, solution addition line through the funnel. & 1.2.2.b & LOW & 1,2 \\
\hline V-M2-3 & MUA & Hayward PVC ball valve, 5 psig air pressure supply line. & 1.2.2.c & LOW & 1,2 \\
\hline V-M2-4 & MUA & Hayward PVC ball valve, outlet line/drain. & 1.2.2.d & LOW & 1,2 \\
\hline V-M2-6 & MUA & Hayward PVC ball valve, solution addition line. & 1.2.2.e & LOW & 1,2 \\
\hline V-M2-7 & MUA & Hayward PVC ball valve, outlet line. & 1.2.2.f & LOW & 1,2 \\
\hline V-M2-11 & MUA & Hayward PVC ball valve, sight glass valve at bottom of sight glass. & 1.2.2.g & LOW & 1,2 \\
\hline V-M2-13 & MUA & Hayward PVC ball valve, outlet line. & 1.2.2.h & LOW & 1,2 \\
\hline Tank T-70 & CUB 7 & 171 L Zr dissolver tank accessible from removable lid in Cubicle 7 floor. & 1.3.1.a & HIGH & 11,12 \\
\hline $\begin{array}{l}\text { T-70 LL, DR, and PR } \\
\text { instr. }\end{array}$ & $\begin{array}{l}\text { CR, MUA, } \\
\text { CUB } 7\end{array}$ & $\begin{array}{l}\text { Foxboro pneumatic instrument is located in the CR and the differential pressure (DP) cell } \\
\text { and associated polyethylene tubing is located in the MUA. The LL, DR, and PR lines are } \\
\text { constructed of Zr and originate in T-70 below Cubicle } 7 \text { floor, travel through the Limited } \\
\text { Access Area (LAA) and are tied to the polyethylene tubing in the MUA. }\end{array}$ & 1.3.1.b & HIGH & $9,10,11,12$ \\
\hline T-70 sparger & $\begin{array}{l}\text { MUA, CR, } \\
\text { CUB } 7\end{array}$ & $\begin{array}{l}\text { Selector switch in CR for high or low flow rate of air, push button switch in CR to activate } \\
\text { solenoid in MUA. In-line orifice controls air flow for low setting. High flow rate is set } \\
\text { manually with a needle valve located in the MUA, and flow rate is indicated on a flow } \\
\text { meter located in the MUA. }\end{array}$ & 1.3.1.c & MED & 5,6 \\
\hline T-70 process lines & CUB 7 & Lines are constructed of $\mathrm{Zr}$ and provide solution transfer routes into and out of T-70. & 1.3.1.d & MED & $4,5,6$ \\
\hline
\end{tabular}




\begin{tabular}{|c|c|c|c|c|c|}
\hline ITEM & LOCATION & DESCRIPTION & Reference & IMPACT & COMMENTS \\
\hline T-70 thermocouple & CUB 7 & Specially fabricated thermocouple that is inserted into the thermo-well in T-70. & 1.3.1.e & HIGH & $9,10,11,12$ \\
\hline P-78 heating loop pump & MUA, CR & $\begin{array}{l}\text { Centrifugal type water pump located in the MUA. There is also an ON/OFF switch in the } \\
\text { CR (Replaced May 2014). }\end{array}$ & 1.3.2.a & HIGH & 9,10 \\
\hline V-M74-1 & MUA & Ball valve, vent line to VOG. & 1.3.2.b & LOW & 1,2 \\
\hline V-M74-2 & MUA & Gate valve, from RCW supply. & 1.3.2.b & LOW & 1,2 \\
\hline V-M74-3 & MUA & Ball valve, 100 psig air supply. & 1.3.2.b & LOW & 1,2 \\
\hline V-M74-10 & MUA & Gate valve, top sight glass valve. & 1.3.2.b & LOW & 1,2 \\
\hline V-M74-12 & MUA & Gate valve, bottom sight glass valve. & 1.3.2.b & LOW & 1,2 \\
\hline V-T555-2 & MUA & Ball valve, P-78 heating/cooling water loop supply to T-555. & 1.3.2.b & LOW & 1,2 \\
\hline V-T555-3 & MUA & Ball valve, P-78 heating/cooling water loop return from T-555. & 1.3.2.b & LOW & 1,2 \\
\hline V-T555-15 & MUA & Ball valve, P-78 heating/cooling water loop drain valve for T-555 loop water. & 1.3.2.b & LOW & 1,2 \\
\hline V-H78-1 & MUA & Gate valve, to RCW return from H-78A heat exchanger. & 1.3.2.b & LOW & 1,2 \\
\hline V-H78-2 & MUA & Gate valve, from RCW supply to H-78A heat exchanger. & 1.3.2.b & LOW & 1,2 \\
\hline V-T70-2 & MUA & Gate valve, P-78 heating/cooling water loop supply to T-70. & 1.3.2.b & LOW & 1,2 \\
\hline V-T70-3 & MUA & Gate valve, P-78 heating/cooling water loop pump discharge to T-70. & 1.3.2.b & LOW & 1,2 \\
\hline V-T70-9 & MUA & Gate valve, P-78 heating/cooling water loop return from T-70. & 1.3.2.b & LOW & 1,2 \\
\hline V-T70-10 & MUA & Gate valve, spare (blanked). & 1.3.2.b & LOW & 1,2 \\
\hline V-T70-11 & MUA & Ball valve, spare (blanked). & 1.3.2.b & LOW & 1,2 \\
\hline V-T70-15 & MUA & Gate valve, P-78 heating/cooling water loop drain valve for T-70 loop water. & 1.3.2.b & LOW & 1,2 \\
\hline PRV-P78-5 & MUA & Pressure relief valve for the P-78 heating/cooling water loop. & 1.3.2.b & LOW & 1,2 \\
\hline Tank M-74 & MUA & Stainless steel tank reservoir for the P-78 the P-78 heating/cooling water loop. & 1.3.2.c & MED & 7 \\
\hline H-78A heat exchanger & MUA & Heat exchanger for the P-78 heating/cooling water loop. & 1.3.2.d & LOW & $2,3,4$ \\
\hline H-78B heater & MUA & Electric heater for heating the water in the P-78 the P-78 heating/cooling water loop. & 1.3.2.d & HIGH & 9,10 \\
\hline TRC-T70 & CR & $\begin{array}{l}\text { Temperature recorder/controller located in the CR controls the operation of TCV-T70 } \\
\text { (Replaced May 2014). }\end{array}$ & 1.3.2.e & MED & $5,6,8$ \\
\hline TCV-T70 & MUA & $\begin{array}{l}\text { Control valve operated by TRC-T70 to control the amount of water cooling allowed into } \\
\text { the water loop to control the heat up and cool down of T-70. }\end{array}$ & 1.3.2.f & LOW & 1,2 \\
\hline FI-T70-2 & MUA & Flow indicator for the P-78 heating/cooling water loop. & 1.3.2.g & LOW & 1,2 \\
\hline FI-T70-3 & MUA & Flow indicator for the RCW water to H-78A. & 1.3.2.g & LOW & 1,2 \\
\hline H-78 thermocouples & MUA & Type K thermocouples measuring the temperature of the P-78 heating/cooling water loop. & 1.3.2.h & LOW & 1,2 \\
\hline Tank M-573 & MUA & $\begin{array}{l}\text { Nominal 9.5 L volume, flanged glass column tank used to deliver } \mathrm{NaOH} \text { solution to T-70 } \\
\text { for dissolving Al HFIR target rods. }\end{array}$ & 1.3.3 & LOW & 1,2 \\
\hline
\end{tabular}




\begin{tabular}{|c|c|c|c|c|c|}
\hline ITEM & LOCATION & DESCRIPTION & Reference & IMPACT & COMMENTS \\
\hline V-M573-1 & MUA & Jamesbury ball valve, vent line to VOG. & 1.3.3.a & LOW & 1,2 \\
\hline V-M573-2 & MUA & Jamesbury ball valve, solution addition line through the funnel. & 1.3.3.b & LOW & 1,2 \\
\hline V-M573-3 & MUA & Jamesbury ball valve, 5 psig air pressure supply line. & 1.3.3.c & LOW & 1,2 \\
\hline V-M573-4 & MUA & Jamesbury ball valve, drain line. & 1.3.3.d & LOW & 1,2 \\
\hline V-M573-6 & MUA & Jamesbury ball valve, 25 psig air pressure supply line. & 1.3.3.e & LOW & 1,2 \\
\hline V-M573-7 & MUA & Jamesbury ball valve, outlet to FCV-M573. & 1.3.3.f & LOW & 1,2 \\
\hline V-M573-9 & MUA & Jamesbury ball valve, discharge from FCV to LR 5. & 1.3.3.g & LOW & 1,2 \\
\hline V-M573-10 & MUA & Jamesbury ball valve, FCV bypass. & 1.3.3.h & LOW & 1,2 \\
\hline V-M573-11 & MUA & Jamesbury ball valve, outlet line from FCV. & 1.3.3.i & LOW & 1,2 \\
\hline V-M573-12 & MUA & Jamesbury ball valve, outlet line from FCV. & 1.3.3.j & LOW & 1,2 \\
\hline V-M573-13 & MUA & Jamesbury ball valve, sample line. & 1.3.3.k & LOW & 1,2 \\
\hline FCV-M573 & MUA & Research Control Valve for flow control during $\mathrm{NaOH}$ addition to T-70. & 1.3.3.1 & MED & 8 \\
\hline FT-M573 & MUA & Differential pressure transmitter for measuring flow rate through FCV-M573. & 1.3.3.m & MED & 8 \\
\hline FIC-M573 & CR & Flow indicator and controller for the $\mathrm{NaOH}$ addition to $\mathrm{T}-70$. & 1.3.3.n & MED & 8 \\
\hline $\begin{array}{l}\text { T7009 gooseneck } \\
\text { (GN), Nozzle L } \\
\end{array}$ & CUB 7 & $\begin{array}{l}\text { T7009 GN (Nozzle L), a standard Zr GN, is an uptake line for T-70 and is located on top of } \\
\text { T-70. }\end{array}$ & 1.4.1.a & LOW & 3,4 \\
\hline T7014 GN & CUB 7 & $\begin{array}{l}\text { T7014 GN, standard Zr GN, is on BR-7 and goes to the Seven position diversion valve } \\
\text { (7PDV)-T70-1. }\end{array}$ & 1.4.1.b & LOW & 3,4 \\
\hline \begin{tabular}{|l|} 
7PDV-T70-1 \\
\end{tabular} & CUB 7 & 7PDV Type B polyethylene block located on BR-7. & 1.4.1.c & LOW & 1,2 \\
\hline F-70 & CUB 7 & Sintered metal filter mounted in position on BR-7. & 1.4.1.d & HIGH & 9,10 \\
\hline FDV-F70 & CUB 7 & $\begin{array}{l}\text { Special polyethylene block for flow diversion valve (FDV) with 3/8 in. ZR tube installed at } \\
\text { blank position in polyethylene' block located on BR-7. }\end{array}$ & 1.4.1.e & LOW & 1,2 \\
\hline PVC valve & CUB 7 & $\begin{array}{l}\text { Haywood PCV ball valves equipped with JACO fitting on each end for use in the hot cell } \\
\text { cubicles. }\end{array}$ & 1.4.1.f & LOW & 1,2 \\
\hline P72010 GN & CUB 7 & T72010 GN, standard Zr GN, is on BR-7 and goes to 7PDV-P720-1. & 1.4.1.f & LOW & 3,4 \\
\hline 7PDV-P720-1 & CUB 7 & 7PDV with Type D polyethylene block located on BR-7. & 1.4.1.g & LOW & 1,2 \\
\hline \begin{tabular}{|l|l} 
Tank T-737 \\
\end{tabular} & CUB 7 & Nominal 7.5 L Ta tank integral to BR-7. & 1.4.1.h & MED & 7 \\
\hline HV-T737-1 position & CUB 7 & Special 1-line block made for HV position located on BR-7. & 1.4.1.i & LOW & 1,2 \\
\hline SA-F111 position & CUB 7 & $\begin{array}{l}\text { Special 1-line polyethylene block with 3/8 in. Zr tubing on one side installed at SA-F111 } \\
\text { position on CUB } 7 \text { sample rack for accessing F-111. }\end{array}$ & 1.4.1.j & LOW & 1,2 \\
\hline V-P70-3 & MUA & Haywood PCV ball valves equipped with JACO fitting on each end for use in the MUA. & 1.4.1.k & LOW & 1,2 \\
\hline HCV-P70-1 & CUB 7 & PVC block toggle hand valve made for HCV positions located on BR-7. & 1.4.1.1 & LOW & 1,2 \\
\hline FDV-P70-1 & CUB 7 & FDV polyethylene block located on BR-7. & 1.4.1.m & LOW & 1,2 \\
\hline Waste tank F-111 & $\begin{array}{l}\text { WASTE } \\
\text { TANK PIT }\end{array}$ & Discussed in Reference 9.4. & 1.4.1.n & $\begin{array}{l}\text { See Ref. } \\
9.2\end{array}$ & See Ref. 9.2 \\
\hline
\end{tabular}




\begin{tabular}{|c|c|c|c|c|c|}
\hline ITEM & LOCATION & DESCRIPTION & Reference & IMPACT & COMMENTS \\
\hline KP-738 & CUB 7 & $\begin{array}{l}\text { Located on RR-7, } 3 \text { in. diameter } \times 6 \text { in. long flanged glass pipe ( } \sim 1 \mathrm{~L} \text { volume) with } \\
\text { fabricated Zr top and bottom plates, KOP for T-737 Transfer System. }\end{array}$ & 1.4.1.o & LOW & 1,2 \\
\hline CU7AJ & CUB 7 & Cubicle 7 air jet used for vacuum source. & 1.4.1.p & LOW & 1,2 \\
\hline Miscellaneous supplies & $\begin{array}{l}\text { CR, MUA, } \\
\text { CUB } 7\end{array}$ & $\begin{array}{l}\text { Polyethylene tubing, male polyethylene ball joints, and plastic compression fittings } \\
\text { (JACOs). }\end{array}$ & 1.4.1.q & LOW & 1,2 \\
\hline Tank M-704 & MUA & $\begin{array}{l}\text { Nominal 5.5 L volume, flanged } 3 \text { in. } \times 48 \text { in. glass column tank used to deliver solutions to } \\
\text { T-70. }\end{array}$ & 1.4 .2 & LOW & 1,2 \\
\hline V-M704-1 & MUA & Jamesbury ball valve, vent line to VOG. & 1.4.2.a & LOW & 1,2 \\
\hline V-M704-2 & MUA & Jamesbury ball valve, solution addition line through the funnel. & 1.4.2.b & LOW & 1,2 \\
\hline V-M704-3 & MUA & Jamesbury ball valve, 5 psig air pressure supply line. & 1.4.2.c & LOW & 1,2 \\
\hline V-M704-4 & MUA & Jamesbury ball valve, drain line/solution outlet. & 1.4.2.d & LOW & 1,2 \\
\hline V-M704-7 & MUA & Jamesbury ball valve, demineralized water addition line. & 1.4.2.e & LOW & 1,2 \\
\hline $\begin{array}{l}\text { Tank M-704 solution } \\
\text { addition system }\end{array}$ & & $\begin{array}{l}\text { Equipment utilized for solution additions to Tank T-70 were previously discussed above } \\
\text { per References 1.4.1.and 1.4.2 above. }\end{array}$ & 1.5 .1 & LOW & 1,2 \\
\hline P-78 heating loop & & $\begin{array}{l}\text { Operation of P-78 heating/cooling water loop was previously discussed above per } \\
\text { Reference 1.3.2 above. }\end{array}$ & 1.5 .2 & \begin{tabular}{|l|} 
See Ref. \\
1.3 .2 \\
\end{tabular} & See Ref. 1.3 .2 \\
\hline $\begin{array}{l}\text { T-70 vacuum } \\
\text { accumulator }\end{array}$ & CUB 7 & $\begin{array}{l}3 \text { in. diameter } \times 6 \text { in. long flanged glass pipe ( } \sim 1 \mathrm{~L} \text { volume) with specially fabricated } \mathrm{Zr} \text { top } \\
\text { and bottom plates. }\end{array}$ & 1.5 .3 & LOW & 1,2 \\
\hline SA-T70 needle block & CUB 7 & Specially fabricated Zr block with two needles for pulling samples from T-70. & 1.5 .3 & LOW & 1,2 \\
\hline $\begin{array}{l}\text { Auxiliary glass sample } \\
\text { flask }\end{array}$ & CUB 7 & $\begin{array}{l}1 \mathrm{~L} \text { glass bottle with 2-line stopper outfitted with polyethylene tubing connected to SA-T70 } \\
\text { position on CUB } 7 \text { sample rack for sampling T-70 and for connection to vacuum source. }\end{array}$ & 1.5 .3 & LOW & 1,2 \\
\hline $\begin{array}{l}\text { SA-T70 special 1-line } \\
\text { block }\end{array}$ & CUB 7 & $\begin{array}{l}\text { Special 1-line polyethylene block with } 3 / 8 \text { in. metal tubing on one side installed at SA-T70 } \\
\text { position on CUB } 7 \text { sample rack for sampling T-70 using auxiliary glass sample flask. }\end{array}$ & 1.5 .3 & LOW & 1,2 \\
\hline T7009 GN—Nozzle L & CUB 7 & $\begin{array}{l}\text { T7009 GN (Nozzle L), standard Zr GN, is an uptake line for T-70 and is located on top of } \\
\text { T-70. }\end{array}$ & 1.6.1.a & LOW & 3,4 \\
\hline F-720 Filter & CUB 7 & $\begin{array}{l}3 \text { in. diameter } \times 10 \text { in. long flanged glass pipe with specially fabricated } \mathrm{Zr} \text { top and bottom } \\
\text { plates, with micron filter element. }\end{array}$ & 1.6.1.b & HIGH & $1,2,3$ \\
\hline F-720 floor base/holder & CUB 7 & Specially fabricated Zr base to hold F-720 filter assembly. & 1.6.1.c & HIGH & $1,2,3$ \\
\hline T-73402 GN & CUB 7 & $\begin{array}{l}\text { Standard Ta GN on RR-7, inlet for T-734 with polyethylene tubing connected to outlet } \\
\text { from F-720. }\end{array}$ & 1.6.1.d & LOW & 1,2 \\
\hline PS-733 position & CUB 7 & Special 2-line block for PS position with polyethylene tubing connecting the two lines. & 1.6.1.e & & \\
\hline HV-T734-2 & CUB 7 & $\begin{array}{l}\text { Special 2-line block (lines are Zr) for HV position with PVC valve and polyethylene tubing } \\
\text { connecting the two lines. }\end{array}$ & 1.6.1.f & LOW & 1,2 \\
\hline
\end{tabular}




\begin{tabular}{|c|c|c|c|c|c|}
\hline ITEM & LOCATION & DESCRIPTION & Reference & IMPACT & COMMENTS \\
\hline Tank T-734 & CUB 7 & Nominal 7.5 L Ta tank integral to BR-7. & 1.6.1.g & MED & 3,4 \\
\hline HV-T734-3 & CUB 7 & $\begin{array}{l}\text { Special 2-line block (lines are Zr) for HV position with PVC valve and polyethylene tubing } \\
\text { connecting the two lines. }\end{array}$ & 1.6.1.h & LOW & 1,2 \\
\hline T73404 GN & CUB 7 & $\begin{array}{l}\text { Standard Ta GN on RR-7, outlet for T-734 with polyethylene tubing connected to T7202 } \\
\text { GN in Cubicle } 7 \text { right DC well. }\end{array}$ & 1.6.1.i & LOW & 1,2 \\
\hline T7202 GN & CUB 7 & Standard Ta GN, is an uptake line for T-72 and is located in Cubicle 7 right DC well. & 1.6.1.j & LOW & 1,2 \\
\hline HV-T734-1 & CUB 7 & $\begin{array}{l}\text { Zr hand valve utilizing a circle seal valve stem fabricated especially for use for use on RR- } \\
7 .\end{array}$ & 1.6.1.k & LOW & 1,2 \\
\hline $\begin{array}{l}\begin{array}{l}\text { KP-735 knock out pot } \\
\text { (KOP) }\end{array} \\
\end{array}$ & CUB 7 & $\begin{array}{l}\text { Located on RR-7, } 3 \text { in. diameter } \times 6 \text { in. long flanged glass pipe ( } \sim 1 \text { L volume) with } \\
\text { specially fabricated Zr top and bottom plates, KOP for T-734 Transfer System. }\end{array}$ & 1.6.1.1 & LOW & 1,2 \\
\hline CU7AJ & CUB 7 & Previously discussed in Reference 1.4.1.p above. & 1.6.1.m & LOW & 1,2 \\
\hline Miscellaneous supplies & $\begin{array}{l}\text { CR, MUA, } \\
\text { CUB } 7\end{array}$ & Previously discussed in Reference 1.4.1.q above. & 1.6.1.n & LOW & 1,2 \\
\hline T-70 Nozzle L & CUB 7 & Previously discussed in Reference 1.6.1.a above. & 1.6.1.0 & LOW & 3,4 \\
\hline F-720 Filter & CUB 7 & Previously discussed in Reference 1.6.1.b above. & 1.6.1.p & HIGH & $9,10,11$ \\
\hline $\begin{array}{l}\text { F-720 floor } \\
\text { base/holder }\end{array}$ & CUB 7 & Previously discussed in Reference 1.6.1.c above. & 1.6.1.q & HIGH & $9,10,11$ \\
\hline F-70 position & CUB 7 & $\begin{array}{l}\text { Special 2-line Zr block [with (2) 3/8 in. diameter Zr lines] in place of F-70 filter to access } \\
\text { the inlet and outlet of F-70 position located on BR-7. }\end{array}$ & 1.6.1.r & LOW & 1,2 \\
\hline FDV-F70 & & Previously discussed in Reference 1.4.1.c above. & 1.6.1.s & LOW & 1,2 \\
\hline P-70 pump & CUB 7 & $\begin{array}{l}\text { Specially designed and fabricated vacuum/pressure diaphragm pump (transuranium [TRU] } \\
\text { pump) located on BR-7 for use in Building } 7920 \text { hot cell cubicles. }\end{array}$ & 1.6.1.t & MED & $5,6,8$ \\
\hline HCV-P70-1 & CUB 7 & Previously discussed in Reference 1.4.1.I above. & 1.6.1.u & LOW & 1,2 \\
\hline FDV-P70-2 & CUB 7 & Previously discussed in Reference 1.4.1.m above. & 1.6.1.v & LOW & 1,2 \\
\hline 7PDV-P730-1 & CUB 7 & Type A polyethylene block located on BR-7. & 1.6.1.w & LOW & 1,2 \\
\hline MDV-P720-2 & CUB 7 & Polyethylene block located on BR-7. & 1.6.1.x & LOW & 1,2 \\
\hline 7PDV-P710-2 & CUB 7 & Type B polyethylene block located on BR-7. & 1.6.1.y & LOW & 1,2 \\
\hline Miscellaneous supplies & $\begin{array}{l}\text { CR, MUA, } \\
\text { CUB } 7\end{array}$ & Previously discussed in Reference 1.4.1.q above. & 1.6.1.z & LOW & 1,2 \\
\hline
\end{tabular}




\begin{tabular}{|c|c|c|c|c|c|}
\hline ITEM & LOCATION & DESCRIPTION & Reference & IMPACT & COMMENTS \\
\hline Tank T-72 & TK PIT 7 & $\begin{array}{l}\text { 74 L Hastelloy C (HC) shell with Ta liner evaporator tank. T-72 has an annulus filled with } \\
\text { Hg for increased heat transfer capability. }\end{array}$ & 1.6.2.a & HIGH & 11,12 \\
\hline $\begin{array}{l}\text { T-72 LL, DR, and PR } \\
\text { instr. }\end{array}$ & $\begin{array}{l}\text { CR, MUA, TK } \\
\text { PT } 7\end{array}$ & $\begin{array}{l}\text { Foxboro pneumatic instrument is located in the CR and the DP cell and associated } \\
\text { polyethylene tubing is located in the MUA. The LL, DR, and PR lines are constructed of Ta } \\
\text { and originate in T-72 in Tank Pit 7, travel through the LAA, and are tied to the } \\
\text { polyethylene tubing that goes to the DP transmitters in the MUA. }\end{array}$ & 1.6.2.b & HIGH & $9,10,11,12$ \\
\hline T-72 sparger & $\begin{array}{l}\text { MUA, CR, TK } \\
\text { PIT } 7\end{array}$ & $\begin{array}{l}\text { There's a push button switch in CR to activate a solenoid in the MUA. An in-line orifice } \\
\text { controls air flow into the tank. }\end{array}$ & 1.6.2.c & LOW & $1,2,4$ \\
\hline $\begin{array}{l}\text { T-72 process and } \\
\text { instrument lines }\end{array}$ & TK PIT 7 & Lines are constructed of Ta and provide solution transfer routes into and out of T-72. & 1.6.2.d & HIGH & $9,10,11,12$ \\
\hline $\begin{array}{l}\text { T-72 vacuum } \\
\text { accumulator }\end{array}$ & CUB 7 & $\begin{array}{l}3 \text { in. diameter } \times 6 \text { in. long flanged glass pipe ( } 1 \mathrm{~L} \text { volume) with specially fabricated } \mathrm{Zr} \text { top } \\
\text { and bottom plates. }\end{array}$ & 1.6.2.e & OSS & \\
\hline SA-T72 needle block & CUB 7 & Specially fabricated Zr block with two needles for pulling samples. & 1.6.2.e & OSS & \\
\hline $\begin{array}{l}\text { Auxiliary glass sample } \\
\text { flask }\end{array}$ & CUB 7 & $\begin{array}{l}1 \mathrm{~L} \text { glass bottle with 2-line stopper outfitted with polyethylene tubing connected to SA-T72 } \\
\text { position on CUB } 7 \text { sample rack for sampling T-72 and for connection to vacuum source. }\end{array}$ & 1.6.2.e & LOW & 1,2 \\
\hline T7202 GN & CUB 7 & Previously discussed in Reference 1.6.1.j above. & 1.6.1.e & LOW & 1,2 \\
\hline T-72 thermocouples & $\begin{array}{l}\text { CR, MUA, TK } \\
\text { PIT } 7\end{array}$ & Thermocouples, leads, and jumpers to Control Room instruments. & 1.6.3.a & HIGH & $9,10,11,12$ \\
\hline $\begin{array}{l}\text { T-72 Steam lines and } \\
\text { valves }\end{array}$ & \begin{tabular}{|l|} 
MUA, CR, TK \\
PIT 7 \\
\end{tabular} & $\begin{array}{l}\text { Steam lines to the tanks, manual Valves in MUA, and electrically operated solenoid valve } \\
\text { in LAA. }\end{array}$ & 1.6.3.b & HIGH & $9,10,11,12$ \\
\hline PIC-T72 & CR & Pressure controller for PCV-T72 that controls steam supply to T-72 heating/cooling jacket. & 1.6.3.c & LOW & 1,2 \\
\hline PCV-T-72 & MUA & Pressure control valve that controls steam supply to T-72 heating/cooling jacket. & 1.6.3.c & LOW & 1,2 \\
\hline $\begin{array}{l}\text { T-72 RCW lines and } \\
\text { valves }\end{array}$ & CR, TK PIT 7 & Manual valves in MUA and lines in LAA. & 1.6.3.d & HIGH & $9,10,11,12$ \\
\hline $\mathrm{H}-72 \mathrm{~A}$ and $\mathrm{H}-72 \mathrm{~B}$ & \begin{tabular}{|l|} 
CR, \\
TK PIT 7
\end{tabular} & Manual valves in MUA and electrically operated solenoid valve in LAA. & 1.6.3.e & HIGH & $9,10,11,12$ \\
\hline T-72 VOG & TK PIT 7 & Condensate moves through VOG line and collects in a condensate collection tank. & 1.6.3.f & LOW & 3 \\
\hline
\end{tabular}


2.0 CLEANEX Operations

\begin{tabular}{|c|c|c|c|c|c|}
\hline ITEM & LOCATION & DESCRIPTION & Reference & IMPACT & COMMENTS \\
\hline Tank M-1 & MUA & Previously discussed in Reference 1.2.1 above. & 2.1 .1 & LOW & 3,4 \\
\hline Tank M-2 & MUA & Previously discussed in Reference 1.2.2 above. & 2.1 .1 & LOW & 3,4 \\
\hline Tank M-3 & MUA & $\begin{array}{l}\text { Nominal } 70 \text { L Penton tank used for cold chemical CLEANEX extractant make up }\{1 M \\
\text { HDEHP[ di-(2-ethylhexyl) phosphoric acid] in NPH }\} \text {. }\end{array}$ & 2.1 .2 & HIGH & 9,10 \\
\hline M-3 agitator & MUA & Greater than 40 year old Graham motor, $90^{\circ}$ gear box, and stirrer. & 2.1 .2 & MED & 5,6 \\
\hline V-M3-1 & MUA & Hayward PVC ball valve, vent line to VOG system. & 2.1.2.a & LOW & 1,2 \\
\hline V-M3-2 & MUA & Hayward PVC ball valve, solution addition line through the funnel. & 2.1.2.b & LOW & 1,2 \\
\hline V-M3-4 & MUA & Hayward PVC ball valve, outlet line/drain from bottom of bull’s eye. & 2.1.2.c & LOW & 1,2 \\
\hline V-M3-13 & MUA & $\begin{array}{l}\text { Hayward PVC ball valve, outlet line/drain from between bottom of the tank and top of the } \\
\text { bull's eye. }\end{array}$ & 2.1.2.d & LOW & 1,2 \\
\hline Tank M-703 & MUA & $\begin{array}{l}\text { Nominal 14.5 L volume, } 4 \text { in. diameter } \times 72 \text { in. tall glass column used for vacuum/transfer } \\
\text { of organic solutions from storage drums to make up tanks. }\end{array}$ & 2.1 .3 & LOW & $1,2,3$ \\
\hline V-M703-1 & MUA & Hayward PVC ball valve, vent line to VOG. & 2.1.3.a & LOW & 1,2 \\
\hline V-M703-2 & MUA & Hayward PVC ball valve, vacuum supply. & 2.1.3.b & LOW & 1,2 \\
\hline V-M703-3 & MUA & Hayward PVC ball valve, 5 psig air pressure supply line. & 2.1.3.c & LOW & 1,2 \\
\hline V-M703-4 & MUA & Hayward PVC ball valve, outlet line/drain from bottom of tank. & 2.1.3.d & LOW & 1,2 \\
\hline V-M703-9 & MUA & Hayward PVC ball valve, HDEHP inlet. & 2.1.3.e & LOW & 1,2 \\
\hline V-M703-10 & MUA & $\begin{array}{l}\text { Hayward PVC ball valve, inlet from HDEHP drum inside flammable storage cabinet in } \\
\text { Room } 212 \text { (drum room). }\end{array}$ & 2.1.3.f & LOW & 1,2 \\
\hline V-C705-1 & MUA & Hayward PVC ball valve, solvent treatment column vent line to VOG. & 2.1.3.g & LOW & 1,2 \\
\hline V-C705-2 & MUA & $\begin{array}{l}\text { Hayward PVC ball valve, inlet from solvent drum inside flammable storage cabinet in } \\
\text { Room } 212 \text { (drum room). }\end{array}$ & 2.1.3.h & LOW & 1,2 \\
\hline V-C705-4 & MUA & Hayward PVC ball valve, outlet from solvent treatment column. & 2.1.3.i & LOW & 1,2 \\
\hline Tank M-70 & MUA & $\begin{array}{l}\text { Nominal } 20 \mathrm{~L} \text { volume, } 6 \text { in. diameter } \times 48 \text { in. tall glass column used for cold ( } \\
\text { nonradiological) chemical additions. }\end{array}$ & 2.1 .4 & LOW & 1,2 \\
\hline V-M70-1 & MUA & Hayward PVC ball valve, vent line to VOG. & 2.1.4.a & LOW & 1,2 \\
\hline V-M70-2 & MUA & Hayward PVC ball valve, solution addition line through funnel. & 2.1.4.b & LOW & 1,2 \\
\hline V-M70-3 & MUA & Hayward PVC ball valve, 5 psig air pressure supply line. & 2.1.4.C & LOW & 1,2 \\
\hline V-M70-4 & MUA & Hayward PVC ball valve, outlet line/drain from bottom of tank. & 2.1.4.d & LOW & 1,2 \\
\hline V-M70-5 & MUA & Hayward PVC ball valve, demineralized water addition line. & 2.1.4.e & LOW & 1,2 \\
\hline V-M70-13 & MUA & Hayward PVC ball valve, outlet line. & 2.1.4.f & LOW & 1,2 \\
\hline
\end{tabular}




\begin{tabular}{|c|c|c|c|c|c|}
\hline ITEM & LOCATION & DESCRIPTION & Reference & IMPACT & COMMENTS \\
\hline Tank M-71 & MUA & $\begin{array}{l}\text { Nominal } 52 \text { L volume, Penton coated tank used for cold (nonradiological) organic chemical } \\
\text { additions. }\end{array}$ & 2.1 .5 & LOW & 1,2 \\
\hline V-M71-1 & MUA & Hayward PVC ball valve, vent line to VOG. & 2.1.5.a & LOW & 1,2 \\
\hline V-M71-2 & MUA & Hayward PVC ball valve, solution addition line through funnel. & 2.1.5.b & LOW & 1,2 \\
\hline V-M71-3 & MUA & Hayward PVC ball valve, 5 psig air pressure supply line. & 2.1.5.c & LOW & 1,2 \\
\hline V-M71-4 & MUA & Hayward PVC ball valve, outlet line/drain from bottom of tank. & 2.1.5.d & LOW & 1,2 \\
\hline V-M71-6 & MUA & Hayward PVC ball valve, inlet line. & 2.1.5.e & LOW & 1,2 \\
\hline V-M71-13 & MUA & Hayward PVC ball valve, outlet line. & 2.1.5.f & LOW & 1,2 \\
\hline Tank M-75 & MUA & $\begin{array}{l}\text { Nominal } 71 \text { L volume, Penton coated tank used for cold (nonradiological) organic chemical } \\
\text { additions. }\end{array}$ & 2.1 .6 & LOW & 1,2 \\
\hline V-M75-1 & MUA & Hayward PVC ball valve, vent line to VOG. & 2.1.6.a & LOW & 1,2 \\
\hline V-M75-2 & MUA & Hayward PVC ball valve, solution addition line through funnel. & 2.1.6.b & LOW & 1,2 \\
\hline V-M75-3 & MUA & Hayward PVC ball valve, 5 psig air pressure supply line. & 2.1.6.c & LOW & 1,2 \\
\hline V-M75-4 & MUA & Hayward PVC ball valve, outlet line/drain from bottom of tank. & 2.1.6.d & LOW & 1,2 \\
\hline V-M75-5 & MUA & Hayward PVC ball valve, demineralized water addition line. & 2.1.6.e & LOW & 1,2 \\
\hline V-M75-10 & MUA & Hayward PVC ball valve, sight glass isolation valve. & 2.1.6.f & LOW & 1,2 \\
\hline V-M75-12 & MUA & Hayward PVC ball valve, inlet line. & 2.1.6.g & LOW & 1,2 \\
\hline V-M75-13 & MUA & Hayward PVC ball valve, outlet line. & 2.1.6.h & LOW & 1,2 \\
\hline Tank T-72 & $\begin{array}{l}\text { CR, MUA, } \\
\text { CUB 7, TK } \\
\text { PIT } 7\end{array}$ & Previously discussed in References 1.6.2 and 1.6.3 above. & 2.2 .1 & $\begin{array}{l}\text { See Ref. } \\
1.6 .2 \text { and } \\
1.6 .3\end{array}$ & $\begin{array}{l}\text { See Ref. 1.6.2 } \\
\text { and 1.6.3 }\end{array}$ \\
\hline Tank M-764 & MUA & $\begin{array}{l}\text { Nominal 9.7 L volume, } 4 \text { in. diameter } \times 48 \text { in. tall glass column used for metering water } \\
\text { into T-72 at a controlled rate. }\end{array}$ & 2.2 .2 & LOW & $1,2,3,4$ \\
\hline V-M764-1 & MUA & Jamesbury or similar style ball valve, vent line to VOG. & 2.2.2.a & LOW & 1,2 \\
\hline V-M764-2 & MUA & Jamesbury or similar style ball valve, solution addition line through the funnel. & 2.2.2.b & LOW & 1,2 \\
\hline V-M764-3 & MUA & Jamesbury or similar style ball valve,- 5-10 psig air pressure supply line. & 2.2.2.c & LOW & 1,2 \\
\hline V-M764-4 & MUA & Jamesbury or similar style ball valve5 drain line/solution outlet. & 2.2.2.d & LOW & 1,2 \\
\hline V-M764-6 & MUA & Jamesbury or similar style ball valve, spare inlet. & 2.2.2.e & LOW & 1,2 \\
\hline V-M764-7 & MUA & Jamesbury or similar style ball valve, outlet to FCV-M764. & 2.2.2.f & LOW & 1,2 \\
\hline V-M764-10 & MUA & Jamesbury or similar style ball valve, FCV bypass. & 2.2.2.g & LOW & 1,2 \\
\hline V-M764-11 & MUA & Jamesbury or similar style ball valve, outlet line. & 2.2.2.h & LOW & 1,2 \\
\hline V-M764-12 & MUA & Jamesbury or similar style ball valve, discharge from FCV-M764 to LR-7. & 2.2.2.i & LOW & 1,2 \\
\hline V-M764-13 & MUA & Jamesbury or similar style ball valve, spare outlet line. & 2.2.2.j & LOW & 1,2 \\
\hline Tank M-704 & MUA & Previously discussed in Reference 1.4.2 above. & 2.2 .3 & LOW & 1,2 \\
\hline
\end{tabular}




\begin{tabular}{|c|c|c|c|c|c|}
\hline ITEM & LOCATION & DESCRIPTION & Reference & IMPACT & COMMENTS \\
\hline Tank M-706 & MUA & $\begin{array}{l}\text { Nominal } 2.4 \mathrm{~L} \text { volume, flanged } 2 \text { in. } \times 48 \text { in. glass column tank used to deliver solutions to } \\
\text { T-70. }\end{array}$ & 2.2 .3 & LOW & 1,2 \\
\hline V-M706-1 & MUA & Jamesbury ball valve, vent line to VOG. & 2.2.3.a & LOW & 1,2 \\
\hline V-M706-2 & MUA & Jamesbury ball valve, solution addition line through the funnel. & 2.2.3.b & LOW & 1,2 \\
\hline V-M706-3 & MUA & \begin{tabular}{|l} 
Jamesbury ball valve- 5-10 psig air pressure supply line. \\
\end{tabular} & 2.2.3.c & LOW & 1,2 \\
\hline V-M706-4 & MUA & Jamesbury ball valve, drain line/solution outlet. & 2.2.3.d & LOW & 1,2 \\
\hline V-M706-7 & MUA & Jamesbury ball valve, demineralized water addition line. & 2.2.3.e & LOW & 1,2 \\
\hline CLEANEX extractant & MUA & $1 M$ HDEHP make up. & 2.3.1.a & LOW & 1,2 \\
\hline $\mathrm{NaOH}$ & MUA & $\mathrm{NaOH}$ make up for acid adjustments. & 2.3.1.a & LOW & 1,2 \\
\hline Tanks M-704 or M-706 & MUA & Previously discussed in References 1.4.2 and 2.2.3 above. & 2.3.1.a & \begin{tabular}{|l|} 
See Ref. \\
1.4 .2 and \\
2.2 .3 \\
\end{tabular} & $\begin{array}{l}\text { See Ref. 1.4.2 } \\
\text { and 2.2.3 }\end{array}$ \\
\hline T-72 sample system & CUB 7 & Previously discussed in Reference 1.6.2.e above. & 2.3.1.b & \begin{tabular}{|l|} 
See Ref. \\
1.6.2.e
\end{tabular} & See Ref. 1.6.2.e \\
\hline FDV-T70-2 & CUB 7 & $\begin{array}{l}\text { Special polyethylene block with 3/8 in. ZR tube installed at blank position on polyethylene } \\
\text { block located on BR-7. }\end{array}$ & 2.3.2.a & LOW & 1,2 \\
\hline T73402 GN & & Previously discussed in Reference 1.6.1.d above. & 2.3.2.b & LOW & 1,2 \\
\hline PS-733 & CUB 7 & $\begin{array}{l}\text { Phase separator is } 3 \text { in. diameter } \times 6 \text { in. long flanged glass pipe ( } \sim 1 \mathrm{~L} \text { volume) with } \\
\text { specially fabricated } \mathrm{Zr} \text { top and bottom plates installed on BR-7. }\end{array}$ & 2.3.2.d & LOW & 1,2 \\
\hline HV-T734-2 & CUB 7 & $\begin{array}{l}\text { Special 2-line block (lines are Zr) for HV position with PVC valve and polyethylene tubing } \\
\text { connecting the two lines. Previously discussed in Reference 1.6.1.f above. }\end{array}$ & 2.3.2.c & LOW & 1,2 \\
\hline Tank T-734 & CUB 7 & Previously discussed in Reference 1.6.1.g above. & 2.3.2.e & MED & 3,4 \\
\hline HV-T734-3 & CUB 7 & $\begin{array}{l}\text { Zr hand valve utilizing a circle seal valve stem fabricated especially for use for use on RR- } \\
\text { 7. Previously discussed in Reference 1.6.1.h above. }\end{array}$ & 2.3.2.f & LOW & 1,2 \\
\hline T73404 GN & CUB 7 & Previously discussed in Reference 1.6.1.i above. & 2.3.2.g & LOW & 1,2 \\
\hline C4C601 GN & CUB 6 & $\begin{array}{l}\text { Standard Zr GN in Cubicle } 6 \text { left DC well with polyethylene tubing connected from } \\
\text { T73404 GN on RR-7. }\end{array}$ & 2.3.2.h & LOW & 1,2 \\
\hline C4C601 jumper & $\begin{array}{l}\text { CUB 6, TK } \\
\text { PIT 6, TK PIT } \\
\text { 5, TK PIT 4, } \\
\text { CUB } 4 \\
\end{array}$ & Hard piped Zr jumper from Cubicle 6 left DC well to Cubicle 4 left DC well. & 2.3.2.i & LOW & $4,11,12$ \\
\hline C4C601 jumper & CUB 4 & Hard piped Zr jumper from Cubicle 4 left DC well to BR-4. & 2.3.2.j & LOW & 4,5 \\
\hline
\end{tabular}




\begin{tabular}{|c|c|c|c|c|c|}
\hline ITEM & LOCATION & DESCRIPTION & Reference & IMPACT & COMMENTS \\
\hline C4C601 GN & CUB 4 & $\begin{array}{l}\text { Standard Zr GN on BR-4 with polyethylene tubing connected to T2302 GN in Cubicle } 4 \\
\text { right DC well. }\end{array}$ & 2.3.2.k & LOW & 1,2 \\
\hline T2302 GN & CUB 4 & $\begin{array}{l}\text { Standard Zr GN in Cubicle } 4 \text { right DC well with polyethylene tubing connected to C4C601 } \\
\text { GN on BR-4. }\end{array}$ & 2.3.2.1 & LOW & 1,2 \\
\hline C7403 GN & CUB 7 & $\begin{array}{l}\text { Standard Zr GN in Cubicle } 7 \text { left DC well with polyethylene tubing connected from } \\
\text { T73404 GN on RR-7. }\end{array}$ & 2.3.2.m & LOW & 1,2 \\
\hline C7403/T2102 jumper & $\begin{array}{l}\text { TK PIT 7/TK } \\
\text { PIT } 6\end{array}$ & C7403 line is teed to T2103 line to T-21 in the Tank Pit. & 2.3.2.m & MED & $4,11,12$ \\
\hline T2103 GN & CUB 6 & Standard Zr GN in Cubicle 6 right DC well with JACO and polyethylene plug. & 2.3.2.n & LOW & 1,2 \\
\hline HV-T734-1 & CUB 7 & Previously discussed in Reference 1.6.1.k above. & 2.3.2.0 & LOW & 1,2 \\
\hline KP-735 KOP & CUB 7 & Previously discussed in Reference 1.6.1.I above. & 2.3.2.p & LOW & 1,2 \\
\hline \begin{tabular}{|l|l} 
CU7AJ \\
\end{tabular} & CUB 7 & Previously discussed in Reference 1.4.1.p above. & 2.3.2.q & LOW & 1,2 \\
\hline Miscellaneous supplies & $\begin{array}{l}\text { CR, MUA, } \\
\text { CUB } 7\end{array}$ & Previously discussed in Reference 1.4.1.q above. & 2.3.2.r & LOW & 1,2 \\
\hline Tank T-21 & \begin{tabular}{|l|} 
TK PIT 2 \\
\end{tabular} & $\sim 155$ L HC tank & 2.3.3.a & HIGH & 11,12 \\
\hline $\begin{array}{l}\text { T-21 instrument and } \\
\text { process lines }\end{array}$ & TK PIT 2 & $\begin{array}{l}\text { Instrument and process lines are constructed of } \mathrm{HC} \text { with the exception of the uptake lines } \\
\text { that are constructed of } \mathrm{Zr} \text {. }\end{array}$ & 2.3.3.b & LOW & 1,2 \\
\hline $\begin{array}{l}\text { T-21 LL, DR, and PR } \\
\text { instr. }\end{array}$ & $\begin{array}{l}\text { CR, MUA, TK } \\
\text { PIT } 2\end{array}$ & $\begin{array}{l}\text { Foxboro pneumatic instrument is located in the CR and the DP cell and associated } \\
\text { polyethylene tubing is located in the MUA. The LL, DR, and PR lines are constructed of Ta } \\
\text { and originate in T-21 in Tank Pit 2, travel through the LAA, and are tied to the } \\
\text { polyethylene tubing that goes to the DP transmitters in the MUA. }\end{array}$ & 2.3.3.c & HIGH & $9,10,11,12$ \\
\hline T-21 sparger & $\begin{array}{l}\text { MUA, CR, TK } \\
\text { PIT } 2\end{array}$ & $\begin{array}{l}\text { There's a push button switch in CR to activate a solenoid in the MUA. An in-line orifice } \\
\text { controls air flow into the tank. }\end{array}$ & 2.3.3.d & LOW & $1,2,4$ \\
\hline $\begin{array}{l}\text { T-21 process and } \\
\text { instrument lines }\end{array}$ & $\begin{array}{l}\text { TK PIT 2, TK } \\
\text { PIT } 6\end{array}$ & $\begin{array}{l}\text { Inlet lines are constructed of } \mathrm{Zr} \text {, and the remainder of the lines are constructed of HC. } \\
\text { These lines provide solution transfer routes into and out of T-21. }\end{array}$ & 2.3.3.e & HIGH & $9,10,11,12$ \\
\hline $\begin{array}{l}\text { T-21 vacuum } \\
\text { accumulator }\end{array}$ & CUB 6 & $\begin{array}{l}3 \text { in. diameter } \times 6 \text { in. long flanged glass pipe ( } \sim 1 \text { L volume) with specially fabricated Zr top } \\
\text { and bottom plates. }\end{array}$ & 2.3.3.f & NCBU & NCBU \\
\hline
\end{tabular}




\begin{tabular}{|c|c|c|c|c|c|}
\hline ITEM & LOCATION & DESCRIPTION & Reference & IMPACT & COMMENTS \\
\hline SA-T21 needle block & CUB 6 & Specially fabricated Zr block with two needles for pulling samples. & 2.3.3.f & NCBU & NCBU \\
\hline $\begin{array}{l}\text { Auxiliary glass sample } \\
\text { flask }\end{array}$ & CUB 6 & $\begin{array}{l}1 \text { L glass bottle with 2-line stopper outfitted with polyethylene tubing connected to SA-T21 } \\
\text { position on CUB } 6 \text { sample rack for sampling T-21 and for connection to vacuum source. }\end{array}$ & 2.3.3.f & LOW & 1,2 \\
\hline $\begin{array}{l}\text { SA-T21 special 1-line } \\
\text { block }\end{array}$ & CUB 6 & $\begin{array}{l}\text { Special 1-line polyethylene block with } 3 / 8 \text { in. metal tubing on one side installed at SA-T21 } \\
\text { position on CUB } 6 \text { sample rack for sampling T-21 using auxiliary glass sample flask. }\end{array}$ & 2.3.3.f & LOW & 1,2 \\
\hline Tank T-23 & TK PIT 2 & $\sim 151 \mathrm{~L}(\mathrm{HC})$ tank. & 2.3.4.a & HIGH & 11,12 \\
\hline $\begin{array}{l}\text { T-23 instrument and } \\
\text { process lines }\end{array}$ & TK PIT 2 & $\begin{array}{l}\text { Instrument and process lines are constructed of HC with the exception of the uptake lines } \\
\text { that are constructed of Zr. }\end{array}$ & 2.3.3.b & LOW & 1,2 \\
\hline $\begin{array}{l}\text { T-23 LL, DR, and PR } \\
\text { instr. }\end{array}$ & $\begin{array}{l}\text { CR, MUA, } \\
\text { CUB } 7\end{array}$ & $\begin{array}{l}\text { Foxboro pneumatic instrument is located in the CR and the DP cell and associated } \\
\text { polyethylene tubing is located in the MUA. The LL, DR, and PR lines are constructed of Ta } \\
\text { and originate in T-23 in Tank Pit 2, travel through the LAA, and are tied to the } \\
\text { polyethylene tubing that goes to the DP transmitters in the MUA. }\end{array}$ & 2.3.4.c & HIGH & $9,10,11,12$ \\
\hline T-23 sparger & $\begin{array}{l}\text { MUA, CR, TK } \\
\text { PIT } 2\end{array}$ & $\begin{array}{l}\text { There's a push button switch in CR to activate a solenoid in the MUA. An in-line orifice } \\
\text { controls air flow into the tank. }\end{array}$ & 2.3.4.d & LOW & $1,2,4$ \\
\hline $\begin{array}{l}\text { T-23 process and } \\
\text { instrument lines }\end{array}$ & \begin{tabular}{|l|} 
TK PIT 2, TK \\
PIT 6 \\
\end{tabular} & $\begin{array}{l}\text { Inlet lines are constructed of } \mathrm{Zr} \text {, and the remainder of the lines are constructed of HC. } \\
\text { These lines provide solution transfer routes into and out of T-23. }\end{array}$ & 2.3.4.e & HIGH & $9,10,11,12$ \\
\hline $\begin{array}{l}\text { T-23 vacuum } \\
\text { accumulator }\end{array}$ & CUB 6 & $\begin{array}{l}3 \text { in. diameter } \times 6 \text { in. long flanged glass pipe ( } \sim 1 \mathrm{~L} \text { volume) with specially fabricated } \mathrm{Zr} \text { top } \\
\text { and bottom plates. }\end{array}$ & 2.3.4.f & OSS & \\
\hline SA-T23 needle block & CUB 6 & Specially fabricated Zr block with 2 needles for pulling samples. & 2.3.4.f & OSS & \\
\hline $\begin{array}{l}\text { Auxiliary glass sample } \\
\text { flask }\end{array}$ & CUB 6 & $\begin{array}{l}1 \text { L glass bottle with 2-line stopper outfitted with polyethylene tubing connected to SA-T23 } \\
\text { position on CUB } 6 \text { sample rack for sampling T-23 and for connection to vacuum source. }\end{array}$ & 2.3.4.f & LOW & 1,2 \\
\hline V-T72-4 & MUA & Haywood PVC ball valve for solution additions into T-72. & 2.3.5.a & LOW & 1,2 \\
\hline $\begin{array}{l}\text { T-72 to T-21/T-23 } \\
\text { Transfer }\end{array}$ & CUB 7, CUB 4 & Previously discussed in Reference 2.3.2 above. & 2.3.5.c & LOW & 1,2 \\
\hline $\begin{array}{l}\text { Tank M-703 transfer } \\
\text { system }\end{array}$ & MUA & Previously discussed in Reference 2.1.3 above. & 2.3.5.d & \begin{tabular}{|l|} 
See Ref. \\
2.1 .3
\end{tabular} & See Ref. 2.1.3 \\
\hline $\begin{array}{l}\text { Tank M-71 phase } \\
\text { modifier addition }\end{array}$ & MUA & $\begin{array}{l}\text { Phase modifier (0.2 M Basic Alamine } 336 \text { in Exxon Aromatic 150) is made up and added to } \\
\text { T-72 from M-71 via V-M71-9 valve. }\end{array}$ & 2.3.5.d & \begin{tabular}{|l|} 
See \\
Ref.2.1.5
\end{tabular} & See Ref.2.1.5 \\
\hline V-T72-4 & MUA & Previously discussed in Reference 2.3.5 above. & 2.3.6.a & \begin{tabular}{|l|} 
See Ref. \\
2.3.5.a
\end{tabular} & See Ref. 2.3.5.a \\
\hline T-72 sample system & CUB 7 & Previously discussed in Reference 1.6.2.e above. & \begin{tabular}{|l|} 
2.3.6.b, \\
2.3.6.c
\end{tabular} & \begin{tabular}{|l} 
See Ref. \\
1.6 .4
\end{tabular} & See Ref. 1.6.4 \\
\hline
\end{tabular}




\begin{tabular}{|c|c|c|c|c|c|}
\hline ITEM & LOCATION & DESCRIPTION & Reference & IMPACT & COMMENTS \\
\hline FDV-T70-2 & CUB 7 & Previously discussed in Reference 2.3.2.a above. & 2.3.7.a & LOW & 1,2 \\
\hline T73402 GN & CUB 7 & $\begin{array}{l}\text { Gooseneck on RR-7, inlet for T-734 with polyethylene tubing connected to left side of } \\
\text { special 2-line block at PS-604 position on BR-6. Reference 1.6.1.d above. }\end{array}$ & 2.3.7.b & LOW & 1,2 \\
\hline PS-733 & CUB 7 & Previously discussed in Reference 2.3.2.c above. & 2.3.7.c & LOW & 1,2 \\
\hline HV-T734-2 & CUB 7 & Previously discussed in Reference 1.6.1.f above. & 2.3.7.c & LOW & 1,2 \\
\hline Tank T-734 & CUB 7 & Previously discussed in Reference 1.6.1.g above. & 2.3.7.e & MED & 3,4 \\
\hline HV-T734-3 & CUB 7 & Previously discussed in Reference 1.6.1.h above. & 2.3.7.f & LOW & 1,2 \\
\hline T73404 GN & CUB 7 & $\begin{array}{l}\text { Gooseneck on RR-7, outlet for T-734 with polyethylene tubing connected to C7405 GN to } \\
\text { T-604. Reference 1.6.1.i above. }\end{array}$ & 2.3.7.g & LOW & 1,2 \\
\hline C7405 GN & CUB 7 & $\begin{array}{l}\text { Standard Ta GN in Cubicle } 7 \text { left DC well (to T-604) with polyethylene tubing connected to } \\
\text { T73404 GN. }\end{array}$ & 2.3.7.h & LOW & 1,2 \\
\hline HV-T734-1 & CUB 7 & Previously discussed in Reference 1.6.1.k above. & 2.3.7.i & LOW & 1,2 \\
\hline KP-735 & CUB 7 & Previously discussed in Reference 1.6.1.I above. & 2.3.7.j & LOW & 1,2 \\
\hline CU7AJ & CUB 7 & Previously discussed in Reference 1.4.1.p above. & 2.3.7.k & LOW & 1,2 \\
\hline Miscellaneous supplies & $\begin{array}{l}\text { CR, MUA, } \\
\text { CUB } 7\end{array}$ & Previously discussed in Reference 1.4.1.q above. & 2.3.7.1 & LOW & 1,2 \\
\hline Tank T-604 & TK PIT 6 & $\begin{array}{l}\text { 22 L HC shell with Ta liner evaporator tank. T-604 has an annulus filled with Hg for } \\
\text { increases heat transfer capability }\end{array}$ & 2.3.8.a & HIGH & 11,12 \\
\hline $\begin{array}{l}\text { T-604 LL, DR, and PR } \\
\text { instr. }\end{array}$ & $\begin{array}{l}\text { CR, MUA, } \\
\text { CUB } 6\end{array}$ & $\begin{array}{l}\text { Foxboro pneumatic instrument is located in the CR and the DP cell and associated } \\
\text { polyethylene tubing is located in the MUA. The LL, DR, and PR lines are constructed of Ta } \\
\text { and originate in T-604 in Tank Pit } 6 \text {, travel through the LAA, and are tied to the } \\
\text { polyethylene tubing that goes to the DP transmitters in the MUA. }\end{array}$ & 2.3.8.b & HIGH & $9,10,11,12$ \\
\hline T-604 sparger & $\begin{array}{l}\text { MUA, CR, TK } \\
\text { PIT } 6\end{array}$ & $\begin{array}{l}\text { There's a push button switch in CR to activate a solenoid in the MUA. An in-line orifice } \\
\text { controls air flow into the tank. }\end{array}$ & 2.3.8.c & LOW & $1,2,4$ \\
\hline $\begin{array}{l}\text { T-604 process and } \\
\text { instrument lines }\end{array}$ & TK PIT 6 & Lines are constructed of Ta and provide solution transfer routes into and out of T-604. & 2.3.8.d & HIGH & $9,10,11,12$ \\
\hline $\begin{array}{l}\text { T-604 vacuum } \\
\text { accumulator }\end{array}$ & CUB 6 & $\begin{array}{l}3 \text { in. diameter } \times 6 \text { in. long flanged glass pipe ( } \sim 1 \mathrm{~L} \text { volume) with specially fabricated } \mathrm{Zr} \text { top } \\
\text { and bottom plates. }\end{array}$ & 2.3.8.e & NCBU & NCBU \\
\hline SA-T604 needle block & CUB 6 & Specially fabricated Zr block with 2 needles for pulling samples. & 2.3.8.e & NCBU & NCBU \\
\hline $\begin{array}{l}\text { Auxiliary glass sample } \\
\text { flask }\end{array}$ & CUB 6 & $\begin{array}{l}1 \text { L glass bottle with 2-line stopper outfitted with polyethylene tubing connected to SA- } \\
\text { T604 position on CUB } 6 \text { sample rack for sampling T-604 and for connection to vacuum } \\
\text { source. }\end{array}$ & 2.3.8.e & LOW & 1,2 \\
\hline
\end{tabular}




\begin{tabular}{|c|c|c|c|c|c|}
\hline ITEM & LOCATION & DESCRIPTION & Reference & IMPACT & COMMENTS \\
\hline $\begin{array}{l}\text { SA-T604 special 1-line } \\
\text { block }\end{array}$ & CUB 6 & $\begin{array}{l}\text { Special 1-line polyethylene block with 3/8 in. metal tubing on one side installed at SA- } \\
\text { T604 position on CUB } 6 \text { sample rack for sampling T-604 using auxiliary glass sample } \\
\text { flask. }\end{array}$ & 2.3.8.e & LOW & 1,2 \\
\hline T-604 thermocouples & $\begin{array}{l}\text { CR, MUA, TK } \\
\text { PIT } 6\end{array}$ & Thermocouples, leads, and jumpers to Control Room instruments. & 2.3.9.a & HIGH & $9,10,11,12$ \\
\hline $\begin{array}{l}\text { T-604 steam lines and } \\
\text { valves }\end{array}$ & $\begin{array}{l}\text { MUA, CR, TK } \\
\text { PIT 6 }\end{array}$ & $\begin{array}{l}\text { Steam lines to the tanks, manual valves in MUA, and electrically operated solenoid valve in } \\
\text { LAA. }\end{array}$ & 2.3.9.b & HIGH & $9,10,11,12$ \\
\hline PIC-T604 & CR & $\begin{array}{l}\text { Pressure controller for PCV-T604 that controls steam supply to T-604 heating/cooling } \\
\text { jacket. }\end{array}$ & 2.3.9.c & LOW & 1,2 \\
\hline PCV-T-604 & MUA & Pressure control valve that controls steam supply to T-604 heating/cooling jacket. & 2.3.9.c & LOW & 1,2 \\
\hline $\begin{array}{l}\text { T-604 RCW lines and } \\
\text { valves }\end{array}$ & CR, TK PIT 6 & Manual Valves in MUA and lines in LAA. & 2.3.9.d & HIGH & $9,10,11,12$ \\
\hline H-604 & CR, TK PIT 6 & Manual Valves in MUA and electrically operated solenoid valve in LAA. & 2.3.9.e & HIGH & $9,10,11,12$ \\
\hline T-604 VOG & TK PIT 6 & Condensate moves through VOG line and collects in a condensate collection tank. & 2.3.9.f & LOW & 3 \\
\hline 7PDV-604-1 & CUB 6 & Type D polyethylene block located on BR-6 (set to 7). & 2.3.10.a & LOW & 1,2 \\
\hline PS-604 & CUB 6 & $\begin{array}{l}\text { Phase separator is } 3 \text { in. diameter } \times 6 \text { in. long flanged glass pipe ( } \sim 1 \mathrm{~L} \text { volume) with } \\
\text { specially fabricated } \mathrm{Zr} \text { top and bottom plates installed on BR-6. }\end{array}$ & 2.3.10.b & LOW & 1,2 \\
\hline HCV-P604-1 & CUB 6 & $\begin{array}{l}\text { 2-line polyethylene block with polyethylene tubing and a PVC ball valve connecting the } \\
\text { two lines. (Polyvinyl chloride block toggle hand valve made for HCV positions may be } \\
\text { installed if desired.) }\end{array}$ & 2.3.10.c & LOW & 1,2 \\
\hline P65301 GN & CUB 6 & $\begin{array}{l}\text { Standard Zr GN on BR-6, inlet for P-653 with polyethylene tubing connected from 2-line } \\
\text { block at HCV-P604-1 position on BR-6. }\end{array}$ & 2.3.10.d & LOW & 1,2 \\
\hline P-653 Pump & CUB 6 & $\begin{array}{l}\text { Specially designed and fabricated vacuum/pressure diaphragm pump (TRU pump) located } \\
\text { on BR-6 for use in Building } 7920 \text { hot cell cubicles. }\end{array}$ & 2.3.10.e & MED & $5,6,8$ \\
\hline P65302 GN & CUB 6 & $\begin{array}{l}\text { Standard Zr GN on BR-6, outlet for P-653 with PVC ball valve and polyethylene tubing } \\
\text { connected to special FDV-T604 on BR-6. }\end{array}$ & 2.3.10.f & LOW & 1,2 \\
\hline FDV-T604 & CUB 6 & FDV Standard polyethylene block located on BR-6. & 2.3.10.g & LOW & 1,2 \\
\hline \begin{tabular}{|l|} 
7PDV-604-2 \\
\end{tabular} & CUB 6 & Seven position diversion valve (7PDV) Type D polyethylene block located on BR-6. & 2.3.10.h & LOW & 1,2 \\
\hline HCV-P604-2 & CUB 6 & $\begin{array}{l}\text { 2-line polyethylene block with polyethylene tubing and a PVC ball valve connecting the } \\
\text { two lines. (Polyvinyl chloride block toggle hand valve made for HCV positions may be } \\
\text { installed if desired.) }\end{array}$ & 2.3.10.i & LOW & 1,2 \\
\hline
\end{tabular}




\begin{tabular}{|c|c|c|c|c|c|}
\hline ITEM & LOCATION & DESCRIPTION & Reference & IMPACT & COMMENTS \\
\hline Miscellaneous supplies & $\begin{array}{l}\text { CR, MUA, } \\
\text { CUB } 6\end{array}$ & Previously discussed in Reference 1.4.1.q above. & 2.3.10.j & LOW & 1,2 \\
\hline Tank T-43 & TK PIT 4 & $\begin{array}{l}\text { 21 L HC shell with Ta liner evaporator tank. T-43 has an annulus filled with Hg for } \\
\text { increases heat transfer capability. }\end{array}$ & 2.3.11.a & HIGH & 11,12 \\
\hline $\begin{array}{l}\text { T-43 LL, DR, and PR } \\
\text { instr. }\end{array}$ & CR, MUA & $\begin{array}{l}\text { Foxboro pneumatic instrument is located in the CR and the DP cell and associated } \\
\text { polyethylene tubing is located in the MUA. The LL, DR, and PR lines are constructed of Ta } \\
\text { and originate in T- } 43 \text { in Tank Pit 4, travel through the LAA, and are tied to the } \\
\text { polyethylene tubing that goes to the DP transmitters in the MUA. }\end{array}$ & 2.3.11.b & HIGH & $9,10,11,12$ \\
\hline T-43 sparger & $\begin{array}{l}\text { MUA, CR, TK } \\
\text { PIT } 4\end{array}$ & $\begin{array}{l}\text { There's a push button switch in CR to activate a solenoid in the MUA. An in-line orifice } \\
\text { controls air flow into the tank. }\end{array}$ & 2.3.11.c & LOW & $1,2,4$ \\
\hline $\begin{array}{l}\text { T-43 process and } \\
\text { instrument lines }\end{array}$ & TK PIT 4 & Lines are constructed of Ta and provide solution transfer routes into and out of T- 43 . & 2.3.11.d & HIGH & $9,10,11,12$ \\
\hline $\begin{array}{l}\text { T-43 vacuum } \\
\text { accumulator }\end{array}$ & CUB 4 & $\begin{array}{l}3 \text { in. diameter } \times 6 \text { in. long flanged glass pipe ( } \sim 1 \mathrm{~L} \text { volume) with specially fabricated } \mathrm{Zr} \text { top } \\
\text { and bottom plates. }\end{array}$ & 2.3.11.e & NCBU & NCBU \\
\hline SA-T43 needle block & CUB 4 & Specially fabricated Zr block with two needles for pulling samples. & 2.3.11.e & NCBU & NCBU \\
\hline $\begin{array}{l}\text { Auxiliary glass sample } \\
\text { flask }\end{array}$ & CUB 4 & $\begin{array}{l}1 \text { L glass bottle with 2-line stopper outfitted with polyethylene tubing connected to SA-T43 } \\
\text { position on CUB } 4 \text { sample rack for sampling T- } 43 \text { and for connection to vacuum source. }\end{array}$ & 2.3.11.e & LOW & 1,2 \\
\hline $\begin{array}{l}\text { SA-T43 special 1-line } \\
\text { block }\end{array}$ & CUB 4 & $\begin{array}{l}\text { Special 1-line polyethylene block with } 3 / 8 \text { in. metal tubing on one side installed at SA-T43 } \\
\text { position on CUB } 4 \text { sample rack for sampling T-43 using auxiliary glass sample flask. }\end{array}$ & 2.3.11.e & LOW & 1,2 \\
\hline T-43 thermocouples & $\begin{array}{l}\text { CR, MUA, TK } \\
\text { PIT } 4\end{array}$ & Thermocouples, leads, and jumpers to Control Room Instruments. & 2.3.12.a & HIGH & $9,10,11,12$ \\
\hline $\begin{array}{l}\text { T-43 steam lines and } \\
\text { valves }\end{array}$ & $\begin{array}{l}\text { MUA, CR, TK } \\
\text { PIT } 4\end{array}$ & $\begin{array}{l}\text { Steam lines to the tanks, manual valves in MUA, and electrically operated solenoid valve in } \\
\text { LAA. }\end{array}$ & 2.3.12.b & HIGH & $9,10,11,12$ \\
\hline PIC-T43 & CR & Pressure controller for PCV-T43 that controls steam supply to T-43 heating/cooling jacket. & 2.3.12.c & LOW & 1,2 \\
\hline PCV-T-43 & MUA & Pressure control valve that controls steam supply to T-43 heating/cooling jacket. & 2.3.12.c & LOW & 1,2 \\
\hline $\begin{array}{l}\text { T-43 RCW lines and } \\
\text { valves }\end{array}$ & CR, TK PIT 4 & Manual valves in MUA and lines in LAA. & 2.3.12.d & HIGH & $9,10,11,12$ \\
\hline $\mathrm{H}-43$ & CR,TK PIT 4 & Manual valves in MUA and electrically operated solenoid valve in LAA. & 2.3.12.e & HIGH & $9,10,11,12$ \\
\hline
\end{tabular}




\begin{tabular}{|c|c|c|c|c|c|}
\hline ITEM & LOCATION & DESCRIPTION & Reference & IMPACT & COMMENTS \\
\hline T-43 VOG & TK PIT 4 & Condensate moves through VOG line and collects in a condensate collection tank. & 2.3.12.f & LOW & 3 \\
\hline $\begin{array}{l}\text { Cubicle } 7 \text { solution } \\
\text { addition line }\end{array}$ & $\begin{array}{l}\text { CUB 7, TK } \\
\text { PIT 7, TK PIT } \\
6\end{array}$ & $\begin{array}{l}\text { Polyethylene tubing from Cubicle } 7 \text { solution addition line connected to C7405 line to T- } \\
604 .\end{array}$ & 2.3.13.a & LOW & 1,2 \\
\hline T-604 sample system & CUB 6 & Previously discussed in Reference 2.3.8.e above. & 2.3.13.b & LOW & 1,2 \\
\hline $\begin{array}{l}\text { T-604 to T-43 transfer } \\
\text { route }\end{array}$ & $\begin{array}{l}\text { CUB 6, TK } \\
\text { PIT 4, TK PIT } \\
6\end{array}$ & Previously discussed in Reference 2.3 .10 above. & 2.3.13.c & LOW & 1,2 \\
\hline T-604 sample system & CUB 6 & Previously discussed in Reference 2.3.8.e above. & 2.3.13.d & LOW & 1,2 \\
\hline T-43 evaporation & $\begin{array}{l}\text { CUB 4, MUA, } \\
\text { TK PIT } 4\end{array}$ & Previously discussed in References 2.3.11 and 2.3.12 above. & 2.3.13.e & $\begin{array}{l}\text { See Ref. } \\
2.3 .11 \text { and } \\
2.3 .12\end{array}$ & $\begin{array}{l}\text { See Ref. 2.3.11 } \\
\text { and 2.3.12 }\end{array}$ \\
\hline T-43 sample system & CUB 4 & Previously discussed in Reference 2.3.11.e above. & 2.3.14.a & LOW & 1,2 \\
\hline $\begin{array}{l}\text { Manometer readings on } \\
\text { T-43 }\end{array}$ & $\begin{array}{l}\text { MUA, TK PIT } \\
4\end{array}$ & $\begin{array}{l}\text { Accountability sampling using a manometer for accurate liquid level and density readings. } \\
\text { Liquid level and density instruments were previously discussed in Reference 2.3.11.b } \\
\text { above. }\end{array}$ & 2.3.14.b & LOW & 1,2 \\
\hline $\begin{array}{l}\mathrm{NaOH} \text { addition to T-72 } \\
\text { and T-604 }\end{array}$ & $\begin{array}{l}\text { MUA, TK PIT } \\
\text { 7, TK PIT } 6\end{array}$ & $\begin{array}{l}\text { M-704 or M-706 is typically used to make the } \mathrm{NaOH} \text { additions via V-M71-9. Previously } \\
\text { discussed in References } \mathbf{1 . 4 . 2} \text { and } \mathbf{2 . 2 . 3} \text { above, respectively. }\end{array}$ & 2.3.15.a & $\begin{array}{l}\text { See Ref. } \\
1.4 .2 \text { and } \\
2.2 .3\end{array}$ & $\begin{array}{l}\text { See Ref. 1.4.2 } \\
\text { and 2.2.3 }\end{array}$ \\
\hline T-72 sample system & CUB 7 & Previously discussed in Reference 1.6.2.e above. & 2.3.15.b & LOW & 1,2 \\
\hline T-604 sample system & CUB 6 & Previously discussed in Reference 2.3.8.e above. & 2.3.15.b & LOW & 1,2 \\
\hline $\begin{array}{l}\text { Tank M-703 } \\
\text { vacuum/pressure } \\
\text { transfer system }\end{array}$ & MUA & Previously discussed in Reference 2.1.3 above. & 2.3.15.d & LOW & 1,2 \\
\hline T-72 waste jet to F-115 & MUA, CR & $\begin{array}{l}\text { T-72 to F-115 waste jet utilizes steam to transfer material to the F-115 tank by opening } \\
\text { manual valves located in the MUA. }\end{array}$ & 2.3.15.e & LOW & 4 \\
\hline
\end{tabular}




\begin{tabular}{|c|c|c|c|c|c|}
\hline ITEM & LOCATION & DESCRIPTION & Reference & IMPACT & COMMENTS \\
\hline $\begin{array}{l}\text { T-604 waste jet to } \\
\text { F-115 }\end{array}$ & MUA, CR & T-604 to F-115 waste jet utilizes steam to transfer material to the F-115 Tank. & 2.3.15.e & LOW & 4 \\
\hline T-72 evaporator & CR, MUA & Previously discussed in References 1.6.2 and 1.6.3 above. & 2.3.15.f & $\begin{array}{l}\text { See Ref. } \\
1.6 .2 \text { and } \\
1.6 .3 \\
\end{array}$ & $\begin{array}{l}\text { See Ref. 1.6.2 } \\
\text { and 1.6.3 }\end{array}$ \\
\hline T-604 evaporator & CR, MUA & Previously discussed in References 2.3.8 and 2.3.9 above. & 2.3.15.f & $\begin{array}{l}\text { See Ref. } \\
2.3 .8 \text { and } \\
2.3 .9\end{array}$ & $\begin{array}{l}\text { See Ref. 2.3.8 } \\
\text { and 2.3.9 }\end{array}$ \\
\hline $\begin{array}{l}\text { T-72 and T-604 waste } \\
\text { jets }\end{array}$ & MUA, CR & Previously discussed in Reference 2.3.15.e above. & 2.3.15.g & LOW & 4 \\
\hline FDV-T70-2 & CUB 7 & Previously discussed in Reference 2.3.2.a above. & 2.3.16.a & LOW & 1,2 \\
\hline T73402 GN & CUB 7 & $\begin{array}{l}\text { Gooseneck on RR-7, inlet for T-734 with polyethylene tubing connected to left side of } \\
\text { special 2-line block at PS-604 position on BR-6. Reference 1.6.1.d above. }\end{array}$ & 2.3.16.b & LOW & 1,2 \\
\hline PS-733 & CUB 7 & Previously discussed in Reference 2.3.2.d above. & 2.3.16.d & LOW & 1,2 \\
\hline HV-T734-2 & CUB 7 & Previously discussed in Reference 1.6.1.f above. & 2.3.16.c & LOW & 1,2 \\
\hline Tank T-734 & CUB 7 & Previously discussed in Reference 1.6.1.g above. & 2.3.16.e & MED & 3,4 \\
\hline HV-T734-3 & CUB 7 & Previously discussed in Reference 1.6.1.h above. & 2.3.16.f & LOW & 1,2 \\
\hline T73404 GN & CUB 7 & $\begin{array}{l}\text { Gooseneck on RR-7, outlet for T-734 with polyethylene tubing connected to 1-line block at } \\
\text { SA-F126 position. Previously discussed in Reference 1.6.1.i above. }\end{array}$ & 2.3.16.g & LOW & 1,2 \\
\hline $\begin{array}{l}\text { SA-F126 special 1-line } \\
\text { block }\end{array}$ & CUB 7 & $\begin{array}{l}\text { Special 1-line polyethylene block with 3/8 in. metal tubing on one side installed at SA-F126 } \\
\text { position on CUB } 7 \text { sample rack for accessing F-126. }\end{array}$ & 2.3.16.h & LOW & 1,2 \\
\hline HV-T734-1 & CUB 7 & Previously discussed in Reference 1.6.1.k above. & 2.3.16.i & LOW & 1,2 \\
\hline KP-735 & CUB 7 & Previously discussed in Reference 1.6.1.I above. & 2.3.16.j & LOW & 1,2 \\
\hline CU7AJ & CUB 7 & Previously discussed in Reference 1.4.1.p above. & 2.3.16.k & LOW & 1,2 \\
\hline Miscellaneous supplies & $\begin{array}{l}\text { CR, MUA, } \\
\text { CUB } 7\end{array}$ & Previously discussed in Reference 1.4.1.q above. & 2.3.16.1 & LOW & 1,2 \\
\hline 7PDV-604-1 & CUB 6 & Previously discussed in Reference 2.3.10.a above & 2.3.17.a & LOW & 1,2 \\
\hline PS-604 position & CUB 6 & $\begin{array}{l}\text { Special 2-line block at PS-604 position with polyethylene tubing connecting the left line to } \\
\text { T73402 GN on RR-7. }\end{array}$ & 2.3.17.b & LOW & 1,2 \\
\hline T73402 GN & CUB 7 & Previously discussed in Reference 1.6.1.d above. & 2.3.17.c & LOW & 1,2 \\
\hline PS-733 & CUB 7 & Previously discussed in Reference 2.3.2.c above. & 2.3.17.e & LOW & 1,2 \\
\hline HV-T734-2 & CUB 7 & Previously discussed in Reference 1.6.1.f above. & 2.3.17.d & LOW & 1,2 \\
\hline
\end{tabular}




\begin{tabular}{|c|c|c|c|c|c|}
\hline ITEM & LOCATION & DESCRIPTION & Reference & IMPACT & COMMENTS \\
\hline Tank T-734 & CUB 7 & Previously discussed in Reference 1.6.1.g above. & 2.3.17.f & MED & 3,4 \\
\hline HV-T734-3 & CUB 7 & Previously discussed in Reference 1.6.1.h above. & 2.3.17.g & LOW & 1,2 \\
\hline T73404 GN & CUB 7 & Previously discussed in Reference 1.6.1.i above. & 2.3.17.h & LOW & 1,2 \\
\hline $\begin{array}{l}\text { SA-F126 special 1-line } \\
\text { block }\end{array}$ & CUB 7 & Previously discussed in Reference 2.3.16.h above. & 2.3.17.i & LOW & 1,2 \\
\hline HV-T734-1 & CUB 7 & Previously discussed in Reference 1.6.1.k above. & 2.3.17.j & LOW & 1,2 \\
\hline KP-735 & CUB 7 & Previously discussed in Reference 1.6.1.I above. & 2.3.17.k & LOW & 1,2 \\
\hline CU7AJ & CUB 7 & Previously discussed in Reference 1.4.1p above. & 2.3.17.1 & LOW & 1,2 \\
\hline Miscellaneous supplies & $\begin{array}{l}\text { CR, MUA, } \\
\text { CUB } 7\end{array}$ & Previously discussed in Reference 1.4.1.q above. & 2.3.17.m & LOW & 1,2 \\
\hline
\end{tabular}


3.0 Anion Exchange Operations

\begin{tabular}{|c|c|c|c|c|c|}
\hline ITEM & LOCATION & DESCRIPTION & Reference & IMPACT & COMMENTS \\
\hline Tank M-600 & MUA & $\begin{array}{l}\text { Nominal } 14.0 \mathrm{~L} \text { volume, flanged glass column tank used to transfer LiCl solution from } \\
\text { drums into make up containers (typically } 25 \mathrm{~L} \text { polyethylene carboys). }\end{array}$ & 3.1 .1 & LOW & 1,2 \\
\hline KOP M-602 & MUA & $\begin{array}{l}2 \text { in. diameter } \times 18 \text { in. long glass column that serves as a KOP to protect the vacuum source } \\
\text { from overfilling M- } 600 \text {. }\end{array}$ & 3.1 .1 & LOW & 1,2 \\
\hline V-M602-1 & MUA & Haywood PVC ball valve, vent line to VOG. & 3.1.1.a & LOW & 1,2 \\
\hline V-M602-2 & MUA & Haywood PVC ball valve, vacuum supply. & 3.1.1.b & LOW & 1,2 \\
\hline V-M602-3 & MUA & Haywood PVC ball valve, 5 psig air pressure supply line. & 3.1.1.c & LOW & 1,2 \\
\hline V-M602-4 & MUA & Haywood PVC ball valve, outlet line/drain line from the bottom of KOP (M-602). & 3.1.1.d & LOW & 1,2 \\
\hline V-M600-4 & MUA & Haywood PVC ball valve, outlet line/drain line from the bottom of the tank (M-600). & 3.1.1.e & LOW & 1,2 \\
\hline V-M600-7 & MUA & Haywood PVC ball valve, inlet line to M-600. & 3.1.1.f & LOW & 1,2 \\
\hline Tank M-63 & MUA & Nominal 1.4 L stainless steel tank used for resin addition to and removal from C-636. & 3.2 .1 & LOW & $1,2,3$ \\
\hline V-M63-1 & MUA & Jamesbury ball valve, vent line to VOG. & 3.2.1.a & LOW & 1,2 \\
\hline V-M63-2 & MUA & Jamesbury ball valve, solution addition line through the funnel. & 3.2.1.b & LOW & 1,2 \\
\hline V-M63-3 & MUA & Jamesbury ball valve, 20 psig air pressure supply line. & 3.2.1.c & LOW & 1,2 \\
\hline V-M63-4 & MUA & Jamesbury ball valve, outlet line/drain. & 3.2.1.d & LOW & 1,2 \\
\hline V-M63-5 & MUA & Jamesbury ball valve, outlet line from FI-M63 to Tank M-63. & 3.2.1.e & LOW & 1,2 \\
\hline V-M63-6 & MUA & Jamesbury ball valve, inlet line to FI-M63. & 3.2.1.f & LOW & 1,2 \\
\hline V-M63-7 & MUA & Jamesbury ball valve, outlet line to the L11 disconnect position in Cubicle 6. & 3.2.1.g & LOW & 1,2 \\
\hline Column C-636 & CUB 6 & $\begin{array}{l}\text { Zr column (Nominal } 2 \text { in. ID } \times 30 \text { in. long) with water jacket for heating/cooling mounted } \\
\text { on RR-6. Typically holds } \sim 1.4 \text { Ls of anion exchange resin for chromatographic separation } \\
\text { of transuranium isotopes. }\end{array}$ & 3.2.1.h & HIGH & $9,10,12$ \\
\hline $\begin{array}{l}\text { Gooseneck at L11 } \\
\text { position in Cubicle } 6\end{array}$ & CUB 6 & Gooseneck with male ferule installed in TRU disconnect. & 3.2.1.i & LOW & 1,2 \\
\hline FDV-C636 & CUB 6 & $\begin{array}{l}\text { Special polyethylene block for FDV with } 3 / 8 \text { in. Zr tube installed at blank position on } \\
\text { polyethylene block located on RR-6. }\end{array}$ & 3.2.1.j & LOW & 1,2 \\
\hline HCV-C636-1 & CUB 6 & $\begin{array}{l}\text { Special } 2 \text { line polyethylene block in the HCV position with (2) 3/8 in. Zr tubes, located on } \\
\text { RR-6. }\end{array}$ & 3.2.1.k & LOW & 1,2 \\
\hline $\begin{array}{l}\text { Cubicle } 6 \\
\text { Demineralized Water }\end{array}$ & CUB 6 & $\begin{array}{l}\text { For resin removal: Cubicle } 6 \text { demineralized water line connected to line on special FDV at } \\
\text { FDV-C636 position on RR-6. }\end{array}$ & 3.2.1.1 & LOW & 1,2 \\
\hline $\begin{array}{l}\text { Polyline from L11 } \\
\text { position in Cubicle } 6\end{array}$ & CUB 6 & $\begin{array}{l}\text { For resin addition: Connect polyethylene line from L11 GN to right side of special 2-line } \\
\text { polyethylene block at HCV-C636-1positon on RR-6. }\end{array}$ & 3.2.1.m & LOW & 1,2 \\
\hline
\end{tabular}




\begin{tabular}{|c|c|c|c|c|c|}
\hline ITEM & LOCATION & DESCRIPTION & Reference & IMPACT & COMMENTS \\
\hline Floor filter assembly & CUB 6 & $\begin{array}{l}\text { Includes standard filter housing for mounting on equipment racks but installed into a floor } \\
\text { mount for versatility of use. }\end{array}$ & 3.2.1.n & LOW & 1,2 \\
\hline $\begin{array}{l}\text { SA-F115 special 1-line } \\
\text { block }\end{array}$ & CUB 7 & $\begin{array}{l}\text { Special 1-line polyethylene block with 3/8 in. Zr metal tubing on one side installed at SA- } \\
\text { F115 position on CUB } 7 \text { sample rack for transferring solution into F-115. }\end{array}$ & 3.2.1.0 & LOW & 1,2 \\
\hline Miscellaneous supplies & $\begin{array}{l}\text { CR, MUA, } \\
\text { CUB 6, } 7\end{array}$ & Previously discussed in Reference 1.4.1.q above. & 3.2.1.p & LOW & 1,2 \\
\hline Tank T-43 & TK PIT 4 & Previously discussed in Reference 2.3.11 and 2.3.12 above. & 3.3 .1 & $\begin{array}{l}\text { See Ref. } \\
2.3 .11 \text {, } \\
2.3 .12 \\
\end{array}$ & \begin{tabular}{|l|} 
See Ref. \\
2.3.11, 2.3.12
\end{tabular} \\
\hline Tanks M-704/M-706 & MUA & Previously discussed in Reference 1.4.2 and 2.2.3 respectively above. & 3.3 .2 & LOW & 1,2 \\
\hline FDV-435 & CUB 4 & $\begin{array}{l}\text { Special polyethylene block for FDV with } 3 / 8 \text { in. Zr tube installed at blank position on } \\
\text { polyethylene block located on BR-4. }\end{array}$ & 3.3.3.a & LOW & 1,2 \\
\hline Floor filter assembly & CUB 6 & $\begin{array}{l}\text { Includes standard filter housing for mounting on equipment racks, but installed into a floor } \\
\text { mount for versatility of use. }\end{array}$ & 3.3.3.b & LOW & 1,2 \\
\hline $\begin{array}{l}\text { Three-prong glass } \\
\text { bottle }\end{array}$ & CUB 6 & 1 L volume glass bottle fitted with a special three-prong stopper assembly. & 3.3.3.c & LOW & 1,2 \\
\hline PVC ball valve & CUB 6 & $\begin{array}{l}\text { Haywood PCV ball valves equipped with JACO fitting on each end for use in the hot cell } \\
\text { cubicles. }\end{array}$ & 3.3.3.c & LOW & 1,2 \\
\hline P65301 GN & CUB 6 & Previously discussed in Reference 2.3.10.d above. & 3.3.3.d & LOW & 1,2 \\
\hline P-653 & CUB 6 & Previously discussed in Reference 2.3.10.e above. & 3.3.3.e & MED & $5,6,8$ \\
\hline P65302 GN & CUB 6 & Previously discussed in Reference 2.3.10.f above. & 3.3.3.f & LOW & 1,2 \\
\hline FDV-T604 & CUB 6 & $\begin{array}{l}\text { Special polyethylene block for FDV with 3/8 in. Zr tube installed at blank position on } \\
\text { polyethylene block located on BR- } 6 \text {. }\end{array}$ & 3.3.3.g & LOW & 1,2 \\
\hline T6503 jumper & CUB 6 & $\begin{array}{l}\text { Zr jumper from BR-6 to Cubicle } 6 \text { left DC well teed with T6509 line from RR-6 and has a } \\
\text { line stub (gooseneck). }\end{array}$ & 3.3.3.h & MED & $4,, 5$ \\
\hline T6503 line stub plug & CUB 6 & $\begin{array}{l}\text { JACO and 3/8 in. polyethylene tubing heat sealed on one end to provide a plug on the } \\
\text { T6503 line stub in Cubicle } 6 \text { floor }\end{array}$ & 3.3.3.i & LOW & 1,2 \\
\hline T6509 line & CUB 6 & Teed to T6503 jumper with a HV in line just prior to the tee into T6503 jumper. & 3.3.3.j & MED & 4,5 \\
\hline HV-T65-2 & CUB 6 & Circle seal type hand valve located in Cubicle 6 floor in line on the T6509 line. & 3.3.3.k & LOW & 1,2 \\
\hline Miscellaneous supplies & $\begin{array}{l}\text { CR, MUA, } \\
\text { CUB 4, } 6\end{array}$ & Previously discussed in Reference 1.4.1.q above. & 3.3.3.1 & LOW & 1,2 \\
\hline
\end{tabular}




\begin{tabular}{|c|c|c|c|c|c|}
\hline ITEM & LOCATION & DESCRIPTION & Reference & IMPACT & COMMENTS \\
\hline Tank M-67 & MUA & $\begin{array}{l}\text { Nominal 9.5 L volume, 4-diameter } \times 48 \text { in. long flanged glass column tank used to transfer } \\
\mathrm{LiCl} \text { solution into Tank Pit tanks and cubicle tanks/columns. }\end{array}$ & 3.4 .1 & LOW & 1,2 \\
\hline V-M67-1 & MUA & Jamesbury ball valve, vent line to VOG. & 3.4.1.a & LOW & 1,2 \\
\hline V-M67-2 & MUA & Jamesbury ball valve, solution addition line through the funnel. & 3.4.1.b & LOW & 1,2 \\
\hline V-M67-3 & MUA & Jamesbury ball valve, 5 psig air pressure supply line. & 3.4.1.C & LOW & 1,2 \\
\hline V-M67-4 & MUA & Jamesbury ball valve, outlet line/drain from the bottom of the tank. & 3.4.1.d & LOW & 1,2 \\
\hline V-M67-6 & MUA & Jamesbury ball valve, 25 psig air pressure supply line. & 3.4.1.e & LOW & 1,2 \\
\hline V-M67-7 & MUA & $\begin{array}{l}\text { Jamesbury ball valve, outlet line to a tee to FCV-M67-8 and V-M67-9 (FCV-M67-8 bypass } \\
\text { line). }\end{array}$ & 3.4.1.f & LOW & 1,2 \\
\hline FCV-M67-8 & MUA & Flow control valve (hardly ever used flow control system). & 3.4.1.g & LOW & 1,2 \\
\hline V-M67-9 & MUA & Jamesbury ball valve, FCV-M67-8 bypass line. & 3.4.1.h & LOW & 1,2 \\
\hline V-M67-11 & MUA & Jamesbury ball valve, outlet line to T-635 inlet. & 3.4.1.i & LOW & 1,2 \\
\hline V-M67-12 & MUA & Jamesbury ball valve, outlet line to T-647 inlet on BR-6. & 3.4.1.j & LOW & 1,2 \\
\hline V-M67-15 & MUA & Jamesbury ball valve, inlet line to FCV-M67-8. & 3.4.1.k & LOW & 1,2 \\
\hline V-M67-16 & MUA & Jamesbury ball valve, outlet line from FCV-M67-8. & 3.4.1.1 & LOW & 1,2 \\
\hline V-M67-17 & MUA & Jamesbury ball valve, outlet line from FCV-M67-8. & 3.4.1.m & LOW & 1,2 \\
\hline Tank T-65 & TK PIT 6 & $\begin{array}{l}\text { 22 L HC shell with Ta liner evaporator tank. T-65 has an annulus filled with Hg for } \\
\text { increased heat transfer capability. }\end{array}$ & 3.4.2.a & HIGH & 11,12 \\
\hline $\begin{array}{l}\text { T-65 LL, DR, and PR } \\
\text { instr. }\end{array}$ & CR, MUA & $\begin{array}{l}\text { Foxboro pneumatic instrument is located in the CR and the DP cell and associated } \\
\text { polyethylene tubing is located in the MUA. The LL, DR, and PR lines are constructed of Ta } \\
\text { and originate in T-65 in Tank Pit } 6 \text {, travel through the LAA, and are tied to the } \\
\text { polyethylene tubing that goes to the DP transmitters in the MUA. }\end{array}$ & 3.4.2.b & HIGH & $9,10,11,12$ \\
\hline T-65 sparger & $\begin{array}{l}\text { MUA, CR, TK } \\
\text { PIT } 6\end{array}$ & $\begin{array}{l}\text { There's a push button switch in CR to activate a solenoid in the MUA. An in-line orifice } \\
\text { controls air flow into the tank. }\end{array}$ & 3.4.2.c & LOW & $1,2,4$ \\
\hline $\begin{array}{l}\text { T-65 process and } \\
\text { instrument lines }\end{array}$ & TK PIT 6 & Lines are constructed of Ta and provide solution transfer routes into and out of T-65. & 3.4.2.d & HIGH & $9,10,11,12$ \\
\hline $\begin{array}{l}\text { T-65 vacuum } \\
\text { accumulator }\end{array}$ & CUB 6 & $\begin{array}{l}3 \text { in. diameter } \times 6 \text { in. long flanged glass pipe ( } \sim 1 \text { L volume) with specially fabricated } \mathrm{Zr} \text { top } \\
\text { and bottom plates. }\end{array}$ & 3.4.2.e & NCBU & NCBU \\
\hline SA-T65 needle block & CUB 6 & Specially fabricated Zr block with 2 needles for pulling samples. & 3.4.2.e & NCBU & NCBU \\
\hline $\begin{array}{l}\text { Auxiliary glass sample } \\
\text { flask }\end{array}$ & CUB 6 & $\begin{array}{l}1 \text { L glass bottle with 2-line stopper outfitted with polyethylene tubing connected to SA-T65 } \\
\text { position on CUB } 6 \text { sample rack for sampling T-65 and for connection to vacuum source. }\end{array}$ & 3.4.2.e & LOW & 1,2 \\
\hline
\end{tabular}




\begin{tabular}{|c|c|c|c|c|c|}
\hline ITEM & LOCATION & DESCRIPTION & Reference & IMPACT & COMMENTS \\
\hline $\begin{array}{l}\text { SA-T65 special 1-line } \\
\text { block }\end{array}$ & CUB 6 & $\begin{array}{l}\text { Special 1-line polyethylene block with 3/8-in metal tubing on one side installed at SA-T65 } \\
\text { position on CUB } 6 \text { sample rack for sampling T-65 using auxiliary glass sample flask. }\end{array}$ & 3.4.2.e & LOW & 1,2 \\
\hline T-65 thermocouples & $\begin{array}{l}\text { CR, MUA, TK } \\
\text { PIT } 6 \\
\end{array}$ & Thermocouples, leads, and jumpers to Control Room instruments. & 3.4.3.a & HIGH & $9,10,11,12$ \\
\hline $\begin{array}{l}\text { T-65 steam lines and } \\
\text { valves }\end{array}$ & $\begin{array}{l}\text { MUA, CR, TK } \\
\text { PIT } 6\end{array}$ & $\begin{array}{l}\text { Steam lines to the tanks, manual valves in MUA, and electrically operated solenoid valve in } \\
\text { LAA. }\end{array}$ & 3.4.3.b & HIGH & $9,10,11,12$ \\
\hline PIC-T65 & CR & Pressure controller for PCV-T65 that controls steam supply to T-65 heating/cooling jacket. & 3.4.3.c & LOW & 1,2 \\
\hline PCV-T-65 & MUA & Pressure control valve that controls steam supply to T-65 heating/cooling jacket. & 3.4.3.c & LOW & 1,2 \\
\hline $\begin{array}{l}\text { T-65 RCW lines and } \\
\text { valves }\end{array}$ & CR, TK PIT 6 & Manual Valves in MUA and lines in LAA. & 3.4.3.d & HIGH & $9,10,11,12$ \\
\hline H-65 & CR,TK PIT 65 & Manual Valves in MUA and electrically operated solenoid valve in LAA. & 3.4.3.e & HIGH & $9,10,11,12$ \\
\hline T-65 VOG & TK PIT 6 & Condensate moves through VOG line and collects in a condensate collection tank. & 3.4.3.f & LOW & 3 \\
\hline P-69 heating loop pump & MUA, CR & $\begin{array}{l}\text { Centrifugal type water pump located in the MUA. There is also an ON/OFF switch in the } \\
\text { CR. }\end{array}$ & 3.4.4.a & HIGH & 9,10 \\
\hline V-P69-1 & MUA & Gate valve, pump discharge to heater inlet. & 3.4.4.b & LOW & 1,2 \\
\hline V-P69-2 & MUA & Gate valve, heater discharge. & 3.4.4.b & LOW & 1,2 \\
\hline V-P69-5 & MUA & Gate valve, return loop water to P-69. & 3.4.4.b & LOW & 1,2 \\
\hline V-P69-6 & MUA & Gate valve, from recirculating cooling water (RCW) supply. & 3.4.4.b & LOW & 1,2 \\
\hline V-P69-7 & MUA & Gate valve, to RCW return. & 3.4.4.b & LOW & 1,2 \\
\hline V-C636-3 & MUA & Gate valve, heater outlet to inlet to C-636. & 3.4.4.b & LOW & 1,2 \\
\hline V-C636-4 & MUA & Gate valve, C-636 outlet to return to pump. & 3.4.4.b & LOW & 1,2 \\
\hline V-C648-3 & MUA & Gate valve, heater outlet to inlet to C-648. & 3.4.4.b & LOW & 1,2 \\
\hline V-C648-4 & MUA & Gate valve, C-648 outlet to return to pump. & 3.4.4.b & LOW & 1,2 \\
\hline V-C56B-14 & MUA & Gate valve, heater outlet to inlet to C56B. & 3.4.4.b & LOW & 1,2 \\
\hline V-C56B-15 & MUA & Gate valve, C56B outlet to return to pump. & 3.4.4.b & LOW & 1,2 \\
\hline V-C56C-14 & MUA & Gate valve, heater outlet to inlet to C56C. & 3.4.4.b & LOW & 1,2 \\
\hline V-C56C-15 & MUA & Gate valve, C56C outlet to return to pump. & 3.4.4.b & LOW & 1,2 \\
\hline PRV-P69-8 & MUA & Pressure relief valve for the P-69 heating/cooling water loop. & 3.4.4.b & LOW & 1,2 \\
\hline H-66 heater & MUA & Electric heaters for heating the water in the P-69 the P-69 heating/cooling water loop. & 3.4.4.c & HIGH & 9,10 \\
\hline
\end{tabular}




\begin{tabular}{|c|c|c|c|c|c|}
\hline ITEM & LOCATION & DESCRIPTION & Reference & IMPACT & COMMENTS \\
\hline TC-T66 & CR & $\begin{array}{l}\text { Temperature recorder and controller located in the CR controls amount of cooling water } \\
\text { allowed into the loop for controlling the heat up and cool down of C-636. }\end{array}$ & 3.4.4.d & MED & $5,6,8$ \\
\hline FI-P69 & MUA & Flow indicator for the P-69 heating/cooling water loop. & 3.4.4.e & LOW & 1,2 \\
\hline H-66 thermocouples & MUA & Type K thermocouples measuring the temperature of the P-69 heating/cooling water loop. & 3.4.4.f & LOW & 1,2 \\
\hline HCV-T635-2 & CUB 6 & PVC block toggle hand valve made for HCV positions located on RR-6. & 3.4.5.a & LOW & 1,2 \\
\hline Tank T-635 & CUB 6 & $\begin{array}{l}3 \text { in. diameter } \times 3 \mathrm{ft} \text { tall flanged glass column located on RR- } 6 \text {. T-635 is used as the feed } \\
\text { tank for the anion exchange column operations. }\end{array}$ & 3.4.5.b & MED & 5,6 \\
\hline P-638 pump & CUB 6 & $\begin{array}{l}\text { Specially designed and fabricated vacuum/pressure diaphragm pump (TRU pump) located } \\
\text { on RR-6, used exclusively for the anion exchange operations. }\end{array}$ & 3.4.5.c & MED & $5,6,8$ \\
\hline HCV-C636-1 & CUB 6 & PVC block toggle hand valve made for HCV positions located on RR-6. & 3.4.5.d & LOW & 1,2 \\
\hline Column C-636 & CUB 6 & Previously discussed in Reference 3.2.1.h above. & 3.4.5.e & HIGH & $9,10,11$ \\
\hline FDV-636 & CUB 6 & FDV polyethylene block located on RR- 6 . & 3.4.5.f & LOW & 1,2 \\
\hline RE-C636-2 & CUB 6 & Position for in-line alpha probe. & 3.4.5.g & LOW & 1,2 \\
\hline HCV-T61 position & CUB 6 & PVC block toggle hand valve made for HCV positions located on RR-6. & 3.4.5.h & LOW & 1,2 \\
\hline HCV-T630 position & CUB 6 & PVC block toggle hand valve made for HCV positions located on RR-6. & 3.4.5.i & LOW & 1,2 \\
\hline HCV-T631 position & CUB 6 & PVC block toggle hand valve made for HCV positions located on RR-6. & 3.4.5.j & LOW & 1,2 \\
\hline HCV-T632 position & CUB 6 & PVC block toggle hand valve made for HCV positions located on RR-6. & 3.4.5.k & LOW & 1,2 \\
\hline HCV-T633 position & CUB 6 & PVC block toggle hand valve made for HCV positions located on RR-6. & 3.4.5.1 & LOW & 1,2 \\
\hline HCV-T634 position & CUB 6 & PVC block toggle hand valve made for HCV positions located on RR-6. & 3.4.5.m & LOW & 1,2 \\
\hline HCV-T65 position & CUB 6 & $\begin{array}{l}\text { Position is out of service due to a leak in the line from C-636 outlet header. The line is } \\
\text { plugged with a special plug to enable the use of the remainder of the header and valves. }\end{array}$ & 3.4.5.n & OOS & OOS \\
\hline HV-T635-1 & CUB 6 & PVC block toggle hand valve made for HV positions located on RR-6. & 3.4.5.0 & LOW & 1,2 \\
\hline RE-C636-1 & CUB 6 & Position for external $\mathrm{n}_{\mathrm{f}}$ probe. & 3.4.5.p & LOW & 1,2 \\
\hline FDV-639 & CUB 6 & FDV polyethylene block located on BR-6. & 3.4.5.q & LOW & 1,2 \\
\hline HCV-T635-2 & CUB 6 & PVC block toggle hand valve made for HCV positions located on RR-6. & 3.4.5.r & LOW & 1,2 \\
\hline HCV-C636-2 & CUB 6 & PVC block toggle hand valve made for HCV positions located on RR-6. & 3.4.5.s & LOW & 1,2 \\
\hline HCV-T635-3 & CUB 6 & PVC block toggle hand valve made for HCV positions located on RR-6. & 3.4.5.t & LOW & 1,2 \\
\hline HV-660 & CUB 6 & PVC block toggle hand valve made for HV positions located on RR-6. & 3.4.5.u & LOW & 1,2 \\
\hline HV-T65-2 & CUB 6 & Previously discussed in Reference 3.3.3.I above. & 3.4.5.v & LOW & 1,2 \\
\hline Miscellaneous supplies & $\begin{array}{l}\text { CR, MUA, } \\
\text { CUB } 6\end{array}$ & Previously discussed in Reference 1.4.1.q above. & 3.4.5.w & LOW & 1,2 \\
\hline Tank T-630 & CUB 6 & Anion Exchange Elution Collection Tank located on RR-6. & 3.4 .6 & HIGH & 11,12 \\
\hline Tank T-631 & CUB 6 & Anion Exchange Elution Collection Tank located on RR-6. & 3.4 .6 & HIGH & 11,12 \\
\hline
\end{tabular}




\begin{tabular}{|c|c|c|c|c|c|}
\hline ITEM & LOCATION & DESCRIPTION & Reference & IMPACT & COMMENTS \\
\hline Tank T-632 & CUB 6 & Anion Exchange Elution Collection Tank located on RR-6. & 3.4 .6 & HIGH & 11,12 \\
\hline Tank T-633 & CUB 6 & Anion Exchange Elution Collection Tank located on RR-6. & 3.4 .6 & HIGH & 11,12 \\
\hline Tank T-634 & CUB 6 & Anion Exchange Elution Collection Tank located on RR-6. & 3.4 .6 & HIGH & 11,12 \\
\hline Tank T-660 & CUB 6 & Knock out tank located on RR-6. & 3.4 .6 & HIGH & 11,12 \\
\hline Tank T-61 & TK PIT 6 & Nominal 67.5 L tank constructed of $\mathrm{Zr}$ located in Tank Pit 6. & 3.4.7.a & HIGH & 11,12 \\
\hline $\begin{array}{l}\text { T-61 LL, DR, and PR } \\
\text { instr. }\end{array}$ & $\begin{array}{l}\text { CR, MUA, } \\
\text { TK PIT } 6\end{array}$ & $\begin{array}{l}\text { Foxboro pneumatic instrument is located in the CR and the DP cell and associated } \\
\text { polyethylene tubing is located in the MUA. The LL, DR, and PR lines are constructed of Ta } \\
\text { and originate in T-61 in Tank Pit } 6 \text {, travel through the LAA, and are tied to the } \\
\text { polyethylene tubing that goes to the DP transmitters in the MUA. }\end{array}$ & 3.4.7.b & HIGH & $9,10,11,12$ \\
\hline T-61 sparger & $\begin{array}{l}\text { MUA, CR, TK } \\
\text { PIT } 6\end{array}$ & $\begin{array}{l}\text { There's a push button switch in CR to activate a solenoid in the MUA. An in-line orifice } \\
\text { controls air flow into the tank. }\end{array}$ & 3.4.7.c & LOW & $1,2,4$ \\
\hline $\begin{array}{l}\text { T-61 process and } \\
\text { instrument lines } \\
\end{array}$ & TK PIT 6 & Lines are constructed of Ta and provide solution transfer routes into and out of T-61. & 3.4.7.d & HIGH & $9,10,11,12$ \\
\hline $\begin{array}{l}\text { T-61 vacuum } \\
\text { accumulator }\end{array}$ & CUB 6 & $\begin{array}{l}3 \text { in. diameter } \times 6 \text { in. long flanged glass pipe ( } \sim 1 \text { L volume) with specially fabricated } \mathrm{Zr} \text { top } \\
\text { and bottom plates. }\end{array}$ & 3.4.7.e & NCBU & NCBU \\
\hline SA-T61 needle block & CUB 6 & Specially fabricated Zr block with two needles for pulling samples. & 3.4.7.e & NCBU & NCBU \\
\hline $\begin{array}{l}\text { Auxiliary glass sample } \\
\text { flask }\end{array}$ & CUB 6 & $\begin{array}{l}1 \text { L glass bottle with 2-line stopper outfitted with polyethylene tubing connected to SA-T61 } \\
\text { position on CUB } 6 \text { sample rack for sampling T-61 and for connection to vacuum source. }\end{array}$ & 3.4.7.e & LOW & 1,2 \\
\hline $\begin{array}{l}\begin{array}{l}\text { SA-T61 special 1-line } \\
\text { block }\end{array} \\
\end{array}$ & CUB 6 & $\begin{array}{l}\text { Special 1-line polyethylene block with 3/8-in metal tubing on one side installed at SA-T61 } \\
\text { position on CUB } 6 \text { sample rack for sampling T-61 using auxiliary glass sample flask. }\end{array}$ & 3.4.7.e & LOW & 1,2 \\
\hline Tank T-72 & $\begin{array}{l}\text { CR, MUA, } \\
\text { CUB 7, TK } \\
\text { PIT 7 } \\
\end{array}$ & Previously discussed in Reference 1.6.2 and 1.6.3 above. & 3.4 .8 & $\begin{array}{l}\text { See Ref. } \\
1.6 .2 \text { and } \\
1.63 \\
\end{array}$ & $\begin{array}{l}\text { See Ref. } 1.6 .2 \\
\text { and } 1.63\end{array}$ \\
\hline Alpha instrumentation & CR & $\begin{array}{l}\text { Utilizes signal from in-cell alpha probe (at RE-C636-2 position) to provide readout and } \\
\text { recorder output for analysis of anion separation operations. }\end{array}$ & 3.4.9.a & HIGH & 9,10 \\
\hline $\begin{array}{l}\text { Neutron } \\
\text { instrumentation }\end{array}$ & CR & $\begin{array}{l}\text { Utilizes signal from in-cell } \mathrm{n}_{\mathrm{f}} \text { probe scan of C-636 column to provide readout and recorder } \\
\text { output for analysis of anion separation progress. }\end{array}$ & 3.4.9.b & HIGH & 9,10 \\
\hline $\begin{array}{l}\text { Miscellaneous jumpers, } \\
\text { connectors, etc. for } \\
\text { alpha and neutron } \\
\text { instrumentation }\end{array}$ & CR & $\begin{array}{l}\text { Various connectors and jumpers required to connect in-cell radiation detectors to } \\
\text { instrumentation in the Control Room. }\end{array}$ & 3.4.9.c & LOW & 1,2 \\
\hline Cubicle drop leads & CUB 6 & $\begin{array}{l}\begin{array}{l}\text { Leads from the cubicle ceiling used to connect the in-cell probes to the instrumentation in } \\
\text { the Control Room. }\end{array} \\
\end{array}$ & 3.4.9.d & MED & 4,6 \\
\hline Tank M-704 & MUA & Previously discussed in Reference 1.4.2 above. & 3.5 .1 & LOW & 1,2 \\
\hline
\end{tabular}




\begin{tabular}{|c|c|c|c|c|c|}
\hline ITEM & LOCATION & DESCRIPTION & Reference & IMPACT & COMMENTS \\
\hline Tank M-706 & MUA & Previously discussed in Reference 2.2.3 above. & 3.5 .1 & LOW & 1,2 \\
\hline Tank T-632 & CUB 6 & Previously discussed in Reference 3.4.6 above. & 3.5 .2 & HIGH & 11,12 \\
\hline HV-639 & CUB 6 & PVC block toggle hand valve made for HV positions located on RR-6. & 3.5.3a & LOW & 1,2 \\
\hline FDV-639 & CUB 6 & Previously discussed in Reference 3.4.5.q above. & 3.5.3.b & LOW & 1,2 \\
\hline T63905 GN & CUB 6 & $\begin{array}{l}\text { Standard Zr GN on RR-6, addition from M-704/M-706 via FDV-639 with polyethylene line } \\
\text { connected to T63207 GN on RR-6. }\end{array}$ & 3.5.3.c & LOW & 1,2 \\
\hline T63207 GN & CUB 6 & $\begin{array}{l}\text { Standard Zr GN on RR-6, uptake line from T-632 with polyline connected to T63905 GN } \\
\text { on RR-6. }\end{array}$ & 3.5.3.c & LOW & 1,2 \\
\hline MDV-637 & CUB 6 & Multiposition diversion valve (MDV) polyethylene block located on RR-6 (set to 2). & 3.5.4.a & LOW & 1,2 \\
\hline HV-637 & CUB 6 & PVC block toggle hand valve made for HV positions located on RR-6. & 3.5.4.b & LOW & 1,2 \\
\hline T63702 GN & CUB 6 & $\begin{array}{l}\text { Standard Zr GN on RR-6, uptake from MDV-637 (from all RR-6 tanks based on MDV } \\
\text { setting) with polyline connected to left side of 2-line block at HCV-P604-1. }\end{array}$ & 3.6.2.c & LOW & 1,2 \\
\hline $\begin{array}{l}\text { Glass frit filter } \\
\text { assembly }\end{array}$ & CUB 6 & Special order medium porosity glass frit filter mounted in special holder. & 3.5.4.c & LOW & 1,2 \\
\hline 1 L KIMEX glass bottle & CUB 6 & $\begin{array}{l}\text { Special } 1 \text { L KIMEX (or PYREX) glass bottle for product collection equipped with rubber } \\
\text { stopper and polyethylene lines. }\end{array}$ & 3.5.4.d & LOW & 1,2 \\
\hline $\begin{array}{l}\text { 1 L KIMEX glass } \\
\text { Bottle }\end{array}$ & CUB 6 & $\begin{array}{l}\text { Special } 1 \text { L KIMEX (or PYREX) glass bottle for intermediate transfer function with rubber } \\
\text { stopper and polyethylene lines. }\end{array}$ & 3.5.4.e & LOW & 1,2 \\
\hline P65301 GN & CUB 6 & Previously discussed in Reference 2.3.10.d above. & 3.5.4.f & LOW & 1,2 \\
\hline P-653 & CUB 6 & Previously discussed in Reference 2.3.10.e above. & 3.5.4.g & MED & $5,6,8$ \\
\hline P65302 GN & CUB 6 & Previously discussed in Reference 2.3.10.f above. & 3.5.4.h & LOW & 1,2 \\
\hline FDV-T604 & CUB 6 & Previously discussed in Reference 3.3.3.g above. & 3.5.4.i & LOW & 1,2 \\
\hline T6503 jumper & CUB 6 & Previously discussed in Reference 3.3.3.h above. & 3.5.4.j & MED & $4,, 5$ \\
\hline T6503 line stub plug & CUB 6 & Previously discussed in Reference 3.3.3.i above. & 3.5.4.k & LOW & 1,2 \\
\hline T6509 line & CUB 6 & Previously discussed in Reference 3.3.3.j above. & 3.5.4.1 & MED & 4,5 \\
\hline HV-T65-2 & CUB 6 & Previously discussed in Reference 3.3.3.k above. & 3.5.4.m & LOW & 1,2 \\
\hline \begin{tabular}{|l|} 
7PDV-604-2 \\
\end{tabular} & CUB 6 & Previously discussed in Reference 2.3.10.h above. & 3.5.4.n & & \\
\hline HCV-P604-2 & CUB 6 & Previously discussed in Reference 2.3.10.i above. & 3.5.4.0 & LOW & 1,2 \\
\hline Miscellaneous supplies & $\begin{array}{l}\text { CR, MUA, } \\
\text { CUB } 6\end{array}$ & Previously discussed in Reference 1.4.1.q above. & 3.5.4.p & LOW & 1,2 \\
\hline Tank T-65 & \begin{tabular}{|l|} 
CR, MUA, \\
CUB 6, TK \\
PIT 6
\end{tabular} & Previously discussed in Reference 3.4.2 and 3.4.3 above. & 3.5 .5 & \begin{tabular}{|l|} 
See Ref. \\
3.4 .2 and \\
3.4 .3 \\
\end{tabular} & $\begin{array}{l}\text { See Ref. 3.4.2 } \\
\text { and 3.4.3 }\end{array}$ \\
\hline MDV-637 & CUB 6 & Previously discussed in Reference 3.5.4.a above (set as required). & 3.6.2.a & LOW & 1,2 \\
\hline HV-637 & CUB 6 & Previously discussed in Reference 3.5.4.b above. & 3.6.2.b & LOW & 1,2 \\
\hline T63702 GN & CUB 6 & Previously discussed in Reference 3.5.4.c above. & 3.6.2.c & LOW & 1,2 \\
\hline P65301 GN & CUB 6 & Previously discussed in Reference 2.3.10.d above. & 3.6.2.d & LOW & 1,2 \\
\hline P-653 & CUB 6 & Previously discussed in Reference 2.3.10.d above. & 3.6.2.e & MED & $5,6,8$ \\
\hline P65302 GN & CUB 6 & Previously discussed in Reference 2.3.10.f above. & 3.6.2.f & LOW & 1,2 \\
\hline
\end{tabular}




\begin{tabular}{|c|c|c|c|c|c|}
\hline ITEM & LOCATION & DESCRIPTION & Reference & IMPACT & COMMENTS \\
\hline C4C601 GN & CUB 6 & Previously discussed in Reference 2.3.2.h above. & 3.6.2.g & LOW & 1,2 \\
\hline C4C601 jumper & $\begin{array}{l}\text { TK PIT 4, 5, } \\
\text { and } 6\end{array}$ & Previously discussed in Reference 2.3.2.i above. & 3.6.2.h & LOW & 1,2 \\
\hline C4C601 jumper & CUB 4 & Previously discussed in Reference 2.3.2.j above. & 3.6.2.i & LOW & 1,2 \\
\hline C4C601 GN & CUB 4 & $\begin{array}{l}\text { Standard Zr GN on BR-4 (previously discussed in Reference 2.3.2.j above) with } \\
\text { polyethylene line connected to T4003 GN. }\end{array}$ & 3.6.2.j & LOW & 1,2 \\
\hline T4003 GN & CUB 4 & $\begin{array}{l}\text { Standard Ta GN in Cubicle } 4 \text { left DC well, discharge to T-40 (also teed to T4013 line in } \\
\text { Cubicle 6) with polyethylene line connected to C4C601 GN. } \\
\end{array}$ & 3.6.2.k & LOW & 1,2 \\
\hline T4013 GN & CUB 6 & $\begin{array}{l}\text { Gooseneck position in Cubicle } 6 \text { left DC well (also teed to T4003 line in Cubicle 6) that is } \\
\text { plugged with a male plug in the DC position in the well. }\end{array}$ & 3.6.2.1 & LOW & 1,2 \\
\hline 7PFDV-604-2 & CUB 6 & Previously discussed in Reference $2.3 .10 . g$ above. & 3.6.2.m & LOW & 1,2 \\
\hline HCV-P604-2 & CUB 6 & Previously discussed in Reference 2.3.10.h above. & 3.6.2.n & LOW & 1,2 \\
\hline Miscellaneous supplies & $\begin{array}{l}\text { CR, MUA, } \\
\text { CUB 4, } 6\end{array}$ & Previously discussed in Reference 1.4.1.q above. & 3.6.2.0 & LOW & 1,2 \\
\hline
\end{tabular}


4.0 Cation Exchange Operations

\begin{tabular}{|c|c|c|c|c|c|}
\hline ITEM & LOCATION & DESCRIPTION & Reference & IMPACT & COMMENTS \\
\hline $\begin{array}{l}\text { Alpha-hydroxyisobuteric } \\
\text { acid (AHIB) }\end{array}$ & $\begin{array}{l}\text { MUA, LAB } \\
110\end{array}$ & Primary elutriate for these operations. Ample quantities are typically keep on hand. & 4.1 .1 & LOW & 3 \\
\hline $\begin{array}{l}\text { Ammonium nitrate } \\
\left(\mathrm{NH}_{4} \mathrm{NO}_{3}\right)\end{array}$ & MUA & Used to treat column resin and miscellaneous adjustments to solutions. & 4.1 .2 & LOW & 3 \\
\hline $\begin{array}{l}\text { Ammonium hydroxide } \\
\left(\mathrm{NH}_{4} \mathrm{OH}\right)\end{array}$ & MUA & Used to adjust $\mathrm{pH}$ of AHIB solutions. & 4.1 .3 & LOW & 3 \\
\hline Polyethylene containers & MUA, LAB & $\begin{array}{l}\text { Several sizes of polyethylene bottles/carboys are used for the chemical make ups for the } \\
\text { cation exchange operations ( } 1 \mathrm{~L}, 8 \mathrm{~L}, 25 \mathrm{~L} \text {, etc.). }\end{array}$ & \begin{tabular}{|l|l|l}
4.1 .1 and \\
4.1 .2 \\
\end{tabular} & LOW & 1,2 \\
\hline Loading column C-402 & CUB 4 & $\begin{array}{l}\text { 5/8 in. diameter } \times 8-3 / 4 \text { in. long stainless steel (SS) tube with Swagelock }{ }^{\circledR} \text { type fitting at } \\
\text { the top and bottom and a SS frit for retaining resin. Located on RR-4. }\end{array}$ & 4.21.a & LOW & 1,2 \\
\hline $\mathrm{P}-407$ & CUB 4 & Positive displacement pump. & 4.2.1.b & LOW & 1,2 \\
\hline $\begin{array}{l}1 / 4 \text { in. polyethylene } \\
\text { tubing with male ball } \\
\text { joint }\end{array}$ & CUB 4 & $1 / 4$ in. diameter polyethylene tubing pre-made in house with male ball joint on one end. & 4.2.1.c & LOW & 1,2 \\
\hline $\begin{array}{l}\text { V-1, V-2, V-3, V-4, V-5, } \\
\text { V-6, V-7, V-8, V-9, V- } \\
10, V-11 \text {, and V-12 }\end{array}$ & CUB 4 & Hoke ${ }^{\circledR}$ brand ball valves, modified specifically for installation and use on RR-4. & 4.2.1.d & LOW & 1,2 \\
\hline PI-407 & CUB 4 & Pressure gauge for P-407 (0-2,000 psig). & 4.2.1.e & LOW & 1,2 \\
\hline RE-401 & CUB 4 & In house fabricated pass through in the alpha detector position on RR-4. & 4.2.1.f & LOW & 1,2 \\
\hline Column wrench & CUB 4 & I in. open end wrench to fit the bottom fitting on Column C-402. & 4.2.1.g & LOW & 1,2 \\
\hline Miscellaneous supplies & $\begin{array}{l}\text { CR, MUA, } \\
\text { CUB } 4\end{array}$ & $\begin{array}{l}\text { Miscellaneous equipment and supplies such as funnels, polyethylene bottles, polyethylene } \\
\text { tubing, bottle holders, etc. }\end{array}$ & 4.2.1.h & LOW & 1,2 \\
\hline DOWEX 50W-X8 resin & CUB 4, MUA & Loading Column C-402 resin. Resin is classified and washed after receipt from vendor. & 4.2 .2 & LOW & 1,2 \\
\hline Loading column C-402 & CUB 4 & Previously discussed in Reference 4.2.1.a above. & 4.2.3.a & LOW & 1,2 \\
\hline Tank T-403 & CUB 4 & $\sim 275 \mathrm{ml}$ volume SS tank equipped with a small funnel located on RR-4. & 4.2.3.b & MED & 5,6 \\
\hline $\begin{array}{l}1 / 4 \text { in. polyethylene } \\
\text { tubing with male ball } \\
\text { joint }\end{array}$ & CUB 4 & Previously discussed in Reference 4.2.1.c above. & 4.2.3.c & LOW & 1,2 \\
\hline $\begin{array}{l}\text { V-1, V-2, V-3, V-4, V-5, } \\
\text { V-6, V-7, V-8, V-9, V- } \\
10, \mathrm{~V}-11 \text {, and V-12 }\end{array}$ & CUB 4 & Previously discussed in Reference 4.2.1.d above. & 4.2.3.d & LOW & 1,2 \\
\hline P-407 & CUB 4 & Previously discussed in Reference 4.2.1.b above. & 4.2.3.e & LOW & 1,2 \\
\hline PI-407 & CUB 4 & Previously discussed in Reference 4.2.1.e above. & 4.2.3.f & LOW & 1,2 \\
\hline Tank T-404 & CUB 4 & $\sim 275$ ml volume SS tank located on RR-4. & 4.2.3.g & MED & 5,6 \\
\hline
\end{tabular}




\begin{tabular}{|c|c|c|c|c|c|}
\hline ITEM & LOCATION & DESCRIPTION & Reference & IMPACT & COMMENTS \\
\hline PI-T404 & CUB 4 & $\begin{array}{l}\text { Pressure/vacuum gauge for T-404 on RR } 4 \text { (range } 0-30 \text { in. water vacuum, } 0-100 \text { psig } \\
\text { pressure). }\end{array}$ & 4.2.3.h & LOW & 1,2 \\
\hline $\begin{array}{l}\text { V-13, V-14, V-15, V- } \\
16 \text {, and V-17 }\end{array}$ & CUB 4 & Hoke ${ }^{\circledR}$ brand ball valves, modified specifically for installation and use on RR-4. & 4.2.3.i & LOW & 1,2 \\
\hline Cubicle 4 vacuum & CUB 4 & Cubicle 4 Air Jet (CU4AJ) for vacuum supply. & 4.2.3.j & LOW & 1,2 \\
\hline PIC-T404 & CR & Pressure controller for PCV-T404 that controls N2pressure to T-404. & 4.2.3.k & LOW & 1,2 \\
\hline PCV-T404 & MUA & Pressure control valve that controls air pressure to T-404. & 4.2.3.1 & LOW & 1,2 \\
\hline PI-T404-2 & MUA & Pressure gauge for pressure supply from PCV-T404 in MUA (range 0-2,000 psig). & 4.2.3.m & LOW & 1,2 \\
\hline $\begin{array}{l}\text { V-T408-1, V-T408-2, } \\
\text { V-T408-3, V-T404-1, } \\
\text { V-T404-2, V-T404-3, } \\
\text { and V-T404-4, }\end{array}$ & MUA & Bonnet needle valves for the $\mathrm{N}_{2}$ pressure station in the MUA. & 4.2.3.n & LOW & 1,2 \\
\hline Miscellaneous supplies & $\begin{array}{l}\text { CR, MUA, } \\
\text { CUB } 4\end{array}$ & Previously discussed in Reference 4.2.1.h above. & 4.2.3.0 & LOW & 1,2 \\
\hline Elution Column C-401 & CUB 4 & $\begin{array}{l}\text { 5/8 in. diameter } \times 48-1 / 8 \text { in. long stainless steel (SS) tubing with Swagelock }{ }^{\circledR} \text { type fitting } \\
\text { at the top and bottom and a SS frit for retaining resin. Column is jacketed with a } 1 \text { in. } \\
\text { diameter } \times 44 \text { in stainless steel tube for heating and cooling water loop. Located on RR-4. }\end{array}$ & 4.3.1.a & HIGH & $9,10,11$ \\
\hline $\begin{array}{l}\text { V-1, V-2, V-3, V-4, V- } \\
\text { 5, V-6, V-7, V-8, V-9, } \\
\text { V-10, V-11, and V-12 }\end{array}$ & CUB 4 & Previously discussed in Reference 4.2.1.d above. & 4.3.1.b & LOW & 1,2 \\
\hline $\begin{array}{l}1 / 4 \text { in. polyethylene } \\
\text { tubing with male ball } \\
\text { joint }\end{array}$ & CUB 4 & Previously discussed in Reference 4.2.1.c above. & 4.3.1.c & & 1,2 \\
\hline $\mathrm{P}-407$ & CUB 4 & Previously discussed in Reference 4.2.1.b above. & 4.3.1.d & LOW & 1,2 \\
\hline PI-407 & CUB 4 & Previously discussed in Reference 4.2.1.e above. & 4.3.1.e & LOW & 1,2 \\
\hline RE-401 & CUB 4 & Previously discussed in Reference 4.2.1.f above. & 4.3.1.f & LOW & 1,2 \\
\hline Column wrench & CUB 4 & Previously discussed in Reference 4.2.1.g above. & 4.3.1.g & LOW & 1,2 \\
\hline Miscellaneous supplies & $\begin{array}{l}\text { CR, MUA, } \\
\text { CUB } 4 \\
\end{array}$ & Previously discussed in Reference 4.2.1.h above. & 4.3.1.h & LOW & 1,2 \\
\hline DOWEX 50W-X8 resin & CUB 4, MUA & Previously discussed in Reference 4.2.2 above. & 4.3 .2 & LOW & 1,2 \\
\hline Elution Column C-401 & CUB 4 & Previously discussed in Reference 4.3.1.a above. & 4.3.3.a & HIGH & $9,10,11$ \\
\hline
\end{tabular}




\begin{tabular}{|c|c|c|c|c|c|}
\hline ITEM & LOCATION & DESCRIPTION & Reference & IMPACT & COMMENTS \\
\hline Tank T-403 & CUB 4 & Previously discussed in Reference 4.2.3.b above. & 4.3.3.b & MED & 5,6 \\
\hline $\begin{array}{l}1 / 4 \text { in. polyethylene } \\
\text { tubing with male ball } \\
\text { joint }\end{array}$ & CUB 4 & Previously discussed in Reference 4.2.1.c above. & 4.3.3.c & LOW & 1,2 \\
\hline $\begin{array}{l}\text { V-1, V-2, V-3, V-4, V- } \\
\text { 5, V-6, V-7, V-8, V-9, } \\
\text { V-10, V-11, and V-12 }\end{array}$ & CUB 4 & Previously discussed in Reference 4.2.1.d above. & 4.3.3.d & LOW & 1,2 \\
\hline $\mathrm{P}-407$ & CUB 4 & Previously discussed in Reference 4.2.1.b above. & 4.3.3.e & LOW & 1,2 \\
\hline PI-407 & CUB 4 & Previously discussed in Reference 4.2.1.e above. & 4.3.3.f & LOW & 1,2 \\
\hline Tank T-404 & CUB 4 & Previously discussed in Reference 4.2.3.g above. & 4.3.3.h & MED & 5,6 \\
\hline PI-T404 & CUB 4 & Previously discussed in Reference 4.2.3.h above. & 4.3.3.i & LOW & 1,2 \\
\hline \begin{tabular}{|l|}
$\mathrm{V}-13, \mathrm{~V}-14, \mathrm{~V}-15, \mathrm{~V}-$ \\
16 , and V-17 \\
\end{tabular} & CUB 4 & Previously discussed in Reference 4.2.3.i above. & 4.3.3.j & LOW & 1,2 \\
\hline Cubicle 4 vacuum & CUB 4 & Previously discussed in Reference 4.2.3.j above. & 4.3.3.k & LOW & 1,2 \\
\hline \begin{tabular}{|l|} 
PIC-T404 \\
\end{tabular} & CR & Previously discussed in Reference 4.2.3.k above. & 4.3.3.1 & LOW & 1,2 \\
\hline PCV-T404 & MUA & Previously discussed in Reference 4.2.3.I above. & 4.3.3.m & LOW & 1,2 \\
\hline PI-T404-2 & MUA & Previously discussed in Reference 4.2.3.m above. & 4.3.3.n & LOW & 1,2 \\
\hline \begin{tabular}{|l|} 
V-T408-1, V-T408-2, \\
V-T408-3, V-T404-1, \\
V-T404-2, V-T404-3, \\
and V-T404-4 \\
\end{tabular} & MUA & Previously discussed in Reference 4.2.3.n above. & 4.3.3.0 & LOW & 1,2 \\
\hline Miscellaneous supplies & $\begin{array}{l}\text { CR, MUA, } \\
\text { CUB } 4 \\
\end{array}$ & Previously discussed in Reference 4.2.1.h above. & 4.3.3.p & LOW & 1,2 \\
\hline $0.3 \mathrm{M} \mathrm{NH}_{4} \mathrm{NO}_{3}$ & MUA, CUB 4 & Stock $\mathrm{NH}_{4} \mathrm{NO}_{3}$, previously made up per Section 4.1.2. & 4.3 .4 & LOW & 1,2 \\
\hline V-H410-1 & MUA & Whitey ball valve, from process water supply. & 4.4.1.a & LOW & 1,2 \\
\hline V-H410-2 & MUA & Whitey ball valve, inlet to $\mathrm{H}-410$. & 4.4.1.a & LOW & 1,2 \\
\hline V-H410-3 & MUA & Whitey ball valve, to drain. & 4.4.1.a & LOW & 1,2 \\
\hline V-H410-4 & MUA & Whitey ball valve, outlet from $\mathrm{H}-410$. & 4.4.1.a & LOW & 1,2 \\
\hline V-H410-5 & MUA & Whitey ball valve, inlet to C-401. & 4.4.1.a & LOW & 1,2 \\
\hline V-P460-6 & MUA & Whitey ball valve, from RCW supply. & 4.4.1.a & LOW & 1,2 \\
\hline PRV-H410 & MUA & Pressure relief valve. & 4.4.1.a & LOW & 1,2 \\
\hline HV-C401-26 & CUB 4 & Gate valve on discharge of H-410 water loop as it exits C-401. & 4.4.1.a & LOW & 1,2 \\
\hline $\mathrm{H}-410$ & MUA & Electric heaters for heating the water in the P-69 the P-69 heating/cooling water loop. & 4.4.1.b & MED & 5,6 \\
\hline
\end{tabular}




\begin{tabular}{|c|c|c|c|c|c|}
\hline ITEM & LOCATION & DESCRIPTION & Reference & IMPACT & COMMENTS \\
\hline TC-H410 & CR & $\begin{array}{l}\text { Temperature Controller located in the CR controls the ON/OFF time to the heater in the } \\
\text { loop for controlling the heat up and cool down of C- } 401 \text {. }\end{array}$ & 4.4.1.c & MED & $5,6.8$ \\
\hline FI-H410 & MUA & Flow indicator for the P-78 heating/cooling water loop. & 4.4.1.d & LOW & 1,2 \\
\hline H-410 thermocouples & MUA & Type K thermocouples measuring the temperature of the $\mathrm{H}-410$ heating/cooling water loop. & 4.4.1.e & MED & 5,6 \\
\hline Tank T-404 & CUB 4 & Previously discussed in Reference 4.2.3.g above. & 4.4.2.a & MED & 5,6 \\
\hline $\begin{array}{l}1 / 4 \text { in. polyethylene } \\
\text { tubing with male ball } \\
\text { joint }\end{array}$ & CUB 4 & Previously discussed in Reference 4.2.1.c above. & 4.4.2.b & LOW & 1,2 \\
\hline $\begin{array}{l}\text { V-1, V-2, V-3, V-4, V- } \\
\text { 5, V-6, V-7, V-8, V-9, } \\
\text { V-10, V-11, and V-12 }\end{array}$ & CUB 4 & Previously discussed in Reference 4.2.1.d above. & 4.4.2.c & LOW & 1,2 \\
\hline $\begin{array}{l}\text { V-13, V-14, V-15, V- } \\
16 \text {, and V-17 }\end{array}$ & CUB 4 & Previously discussed in Reference 4.2.3.i above. & 4.4.2.d & LOW & 1,2 \\
\hline Cubicle 4 vacuum & CUB 4 & Previously discussed in Reference 4.2.1.j above. & 4.4.2.e & LOW & 1,2 \\
\hline RE-401 & CUB 4 & Previously discussed in Reference 4.2.1.f above. & 4.4.2.f & LOW & 1,2 \\
\hline PIC-T404 & CR & Previously discussed in Reference 4.2.3.k above. & 4.4.2.g & LOW & 1,2 \\
\hline PCV-T404 & MUA & Previously discussed in Reference 4.2.3.I above. & 4.4.2.h & LOW & 1,2 \\
\hline PI-T404-2 & MUA & Previously discussed in Reference 4.2.3.m above. & 4.4.2.i & LOW & 1,2 \\
\hline $\begin{array}{l}\text { V-T408-1, V-T408-2, } \\
\text { V-T408-3, V-T404-1, } \\
\text { V-T404-2, V-T404-3, } \\
\text { and V-T404-4 }\end{array}$ & MUA & Previously discussed in Reference 4.2.3.n above. & 4.4.2.j & LOW & 1,2 \\
\hline Miscellaneous supplies & $\begin{array}{l}\text { CR, MUA, } \\
\text { CUB } 4\end{array}$ & Previously discussed in Reference 4.2.1.h above. & 4.4.2.k & LOW & 1,2 \\
\hline $\begin{array}{l}\mathrm{H}_{2} \mathrm{O} \text { flush and } 0.3 \mathrm{M} \\
\mathrm{NaNO}_{3} \text { conditioning }\end{array}$ & CUB 4, MUA & $\begin{array}{l}0.3 M \mathrm{NaNO}_{3} \text { solution and route previously discussed in Sections } 4.1 .2 \text { and 4.4.2 } \\
\text { respectively above. }\end{array}$ & 4.4 .3 & LOW & 1,2 \\
\hline Loading column C-402 & CUB 4 & Previously discussed in Reference 4.2.1.a above. & 4.5.1.a & LOW & 1,2 \\
\hline Elution Column C-401 & CUB 4 & Previously discussed in Reference 4.3.1.a above. & 4.5.1.b & HIGH & $9,10,11$ \\
\hline $\begin{array}{l}1 / 4 \text { in. polyethylene } \\
\text { tubing with male ball } \\
\text { joint }\end{array}$ & CUB 4 & Previously discussed in Reference 4.4.2.b above. & 4.5.1.c & LOW & 1,2 \\
\hline $\begin{array}{l}\text { V-1, V-2, V-3, V-4, V- } \\
\text { 5, V-6, V-7, V-8, V-9, } \\
\text { V-10, V-11, and V-12 }\end{array}$ & CUB 4 & Previously discussed in Reference 4.2.1.d above. & 4.5.1.d & LOW & 1,2 \\
\hline
\end{tabular}




\begin{tabular}{|c|c|c|c|c|c|}
\hline ITEM & LOCATION & DESCRIPTION & Reference & IMPACT & COMMENTS \\
\hline $\mathrm{P}-407$ & CUB 4 & Previously discussed in Reference 4.2.1.b above. & 4.5.1.e & LOW & 1,2 \\
\hline PI-407 & CUB 4 & Previously discussed in Reference 4.2.1.e above. & 4.5.1.f & LOW & 1,2 \\
\hline RE-401 & CUB 4 & Previously discussed in Reference 4.2.1.f above. & 4.5.1.g & LOW & 1,2 \\
\hline $\begin{array}{l}\text { Special Tygon } \\
\text { discharge line }\end{array}$ & CUB 4 & Tygon ${ }^{\circledR}$ discharge line with SS end piece bent into a hook. & 4.5.1.h & LOW & 1,2 \\
\hline $\begin{array}{l}250 \mathrm{ml} \text { polyethylene } \\
\text { bottles }\end{array}$ & CUB 4 & Approximately 25 calibrated and labeled polyethylene bottles to collect product cuts. & 4.5.1.i & LOW & 1,2 \\
\hline $\begin{array}{l}\text { 1/8 in. stainless steel } \\
\text { dip line }\end{array}$ & CUB 4 & $\begin{array}{l}1 / 8 \text { in. stainless steel tubing with female ball joint attached to one end, used for solution } \\
\text { transfers from bottles. }\end{array}$ & 4.5.1.j & LOW & 1,2 \\
\hline Miscellaneous supplies & $\begin{array}{l}\text { CR, MUA, } \\
\text { CUB } 4\end{array}$ & Previously discussed in Reference 4.2.1.h above. & 4.5.1.k & LOW & 1,2 \\
\hline Alpha instrumentation & CR & $\begin{array}{l}\text { Utilizes signal from in-cell alpha probe (at RE-C401 position) to provide readout and } \\
\text { recorder output for analysis of cation separation operations. }\end{array}$ & 4.5.2.a & HIGH & 9,10 \\
\hline $\begin{array}{l}\text { Neutron } \\
\text { instrumentation }\end{array}$ & CR & $\begin{array}{l}\text { Utilizes signal from in-cell } \mathrm{n}_{\mathrm{f}} \text { probe scan of C- } 401 \text { and } \mathrm{C}-402 \text { columns to provide readout } \\
\text { and recorder output for analysis of cation separation progress. }\end{array}$ & 4.5.2.b & HIGH & 9,10 \\
\hline $\begin{array}{l}\text { Miscellaneous jumpers, } \\
\text { connectors, etc. for } \\
\text { alpha and neutron } \\
\text { instrumentation }\end{array}$ & CR & $\begin{array}{l}\text { Various connectors and jumpers required to connect in-cell radiation detectors to } \\
\text { instrumentation in the Control Room. }\end{array}$ & 4.5.2.c & LOW & 1,2 \\
\hline Cubicle drop leads & CUB 6 & $\begin{array}{l}\text { Leads from the cubicle ceiling used to connect the in-cell probes to the instrumentation in } \\
\text { the Control Room. }\end{array}$ & 4.5.2.d & MED & 4,6 \\
\hline $\begin{array}{l}\text { Small volume glass } \\
\text { pipettes and rubber } \\
\text { bulbs }\end{array}$ & CUB 4 & Approximately $5 \mathrm{ml}$ glass pipettes with rubber bulbs to fit the size of the pipette. & $\begin{array}{l}4.6 .1 \text { and } \\
4.6 .2\end{array}$ & LOW & 1,2 \\
\hline $\begin{array}{l}\text { Loading column C-402 } \\
\text { resin removal }\end{array}$ & CUB 4 & Discussed in Section 4.2. & 4.7.1 & $\begin{array}{l}\text { See Ref. } \\
4.2\end{array}$ & See Ref. 4.2 \\
\hline $\begin{array}{l}\text { Elution Column C-401 } \\
\text { resin removal }\end{array}$ & CUB 4 & Discussed in Section 4.3. & 4.7 .2 & $\begin{array}{l}\text { See Ref. } \\
4.3\end{array}$ & See Ref. 4.3 \\
\hline 2 L polyethylene bottle & CUB 4 & $\begin{array}{l}\text { Calibrated and labeled } 2 \mathrm{~L} \text { polyethylene bottle used for compositing selected }{ }^{252} \mathrm{Cf} \text { products } \\
\text { cuts. }\end{array}$ & 4.7 .3 & LOW & 1,2 \\
\hline Small glass funnel & CUB 4 & $\begin{array}{l}\text { Typically a PYREX or KIMEX glass funnel with a stem sized to fit into the mouth of the } \\
2 \text { L product composite bottle. }\end{array}$ & 4.7 .3 & LOW & 1,2 \\
\hline $\begin{array}{l}\text { Recycle cuts } \\
\text { disposition }\end{array}$ & $\begin{array}{l}\text { CUB } 4, \text { TK } \\
\text { PIT } 4, \text { TK PIT } \\
6 \text { or } 7\end{array}$ & $\begin{array}{l}\text { Routes for disposition of recycle cuts discussed in Sections 9.2.8, 10.2.1, 10.2.2, 2.3.3, and } \\
\text { 2.3.4. }\end{array}$ & 4.7 .4 & \begin{tabular}{l|} 
See Ref. \\
$9.2 .8,10.2$. \\
$1,10.3 .1,2$ \\
$2.3,2.3 .4$ \\
\end{tabular} & $\begin{array}{l}\text { See Ref. } \\
9.2 \cdot 8,10.2 \cdot 1,10 . \\
3.1,2.2 .3,2.3 .4\end{array}$ \\
\hline $\begin{array}{l}\text { Waste solution } \\
\text { disposition }\end{array}$ & $\begin{array}{l}\text { CUB } 4, \text { TK } \\
\text { PIT } 4, \text { TK PIT } \\
6 \text { or } 7 \\
\end{array}$ & $\begin{array}{l}\text { Routes for disposition of waste solutions discussed previously in Sections 9.2.8, 10.2.1, } \\
\text { and 10.2.2. }\end{array}$ & 4.7 .5 & \begin{tabular}{l|} 
See Ref. \\
$9.2 .8,10.2$ \\
$1,10.3 .1$ \\
\end{tabular} & $\begin{array}{l}\text { See Ref. } \\
9.2 .8,10.2 .1,10 . \\
3.1 \\
\end{array}$ \\
\hline
\end{tabular}


5.0 Californium Packaging

\begin{tabular}{|c|c|c|c|c|c|}
\hline ITEM & LOCATION & DESCRIPTION & Reference & IMPACT & COMMENTS \\
\hline Pt capsules & \begin{tabular}{|l|} 
STORAGE, \\
CUB 4
\end{tabular} & $\begin{array}{l}\text { 3/8 in. diameter } \times 1-3 / 16 \text { in. long Pt tubes with Pt frits on each end loaded with ion } \\
\text { exchange resin. }\end{array}$ & 5.1.1.a & LOW & 1,2 \\
\hline Cf nut packages & CUB 4 & Swagelock® nuts welded in a configuration that captures the Pt capsule. & 5.1.1.b & LOW & 1,2 \\
\hline Tank T-408 & CUB 4 & $\begin{array}{l}\sim 275 \mathrm{ml} \text { volume stainless steel tank on RR-4 is the feed tank for the Cf Package loading } \\
\text { operations. }\end{array}$ & 5.1.2.a & MED & 5,6 \\
\hline Tank T-405 & CUB 4 & $\begin{array}{l}\sim 500 \mathrm{ml} \text { volume stainless steel tank on RR-4 is the vacuum tank used as a vacuum source } \\
\text { for T- } 408 \text { for making feed transfers. }\end{array}$ & 5.1.2.b & MED & 5,6 \\
\hline $\begin{array}{l}1 / 4 \text { in. polyethylene } \\
\text { tubing with male ball } \\
\text { joint }\end{array}$ & CUB 4 & 1/4 in. diameter polyethylene tubing pre-made in house with male ball joint on one end. & 5.1.2.c & LOW & 5,6 \\
\hline $\begin{array}{l}\text { V-13, V-14, V-15, V- } \\
16 \text {, and V-17 }\end{array}$ & CUB 4 & Previously discussed in Reference 4.2.3.i above. & 5.1.2.d & LOW & 1,2 \\
\hline PI-T408-1 & CUB 4 & Pressure gauge for T-408 (range 0-30 in. Hg vacuum; 0-500 psi pressure). & 5.1.2.e & LOW & 1,2 \\
\hline Cubicle 4 vacuum & CUB 4 & Previously discussed in Reference 4.2.1.j above. & 5.1.2.f & LOW & 1,2 \\
\hline PIC-T404 & CR & Previously discussed in Reference 4.2.3.k above. & 5.1.2.g & LOW & 1,2 \\
\hline PCV-T404 & MUA & Previously discussed in Reference 4.2.3.I above. & 5.1.2.h & LOW & 1,2 \\
\hline PI-T404-2 & MUA & Previously discussed in Reference 4.2.3.m above. & 5.1.2.i & LOW & 1,2 \\
\hline $\begin{array}{l}\text { HS-T408-4 and } \\
\text { HS-T408-5 }\end{array}$ & CR & $\begin{array}{l}\text { Switches located on Control Room panel board MB-23 that respectively increase and } \\
\text { decrease the pressure supply to T- } 408 \text {. }\end{array}$ & 5.1.2.j & LOW & 1,2 \\
\hline PCV-T408 & MUA & Pressure control valve that controls air pressure to T-408. & 5.1.2.k & LOW & 1,2 \\
\hline PI-T408-2 & MUA & Pressure gauge for pressure supply from PCV-T408 in MUA (range $0-2,000$ psig). & 5.1.2.1 & LOW & 1,2 \\
\hline $\begin{array}{l}\text { V-T408-1, V-T408-2, } \\
\text { V-T408-3, V-T404-1, } \\
\text { V-T404-2, V-T404-3, } \\
\text { and V-T404-4 }\end{array}$ & MUA & Previously discussed in Reference 4.2.3.n above. & 5.1.2.m & LOW & 1,2 \\
\hline Miscellaneous supplies & $\begin{array}{l}\text { CR, MUA, } \\
\text { CUB } 4\end{array}$ & Previously discussed in Reference 4.2.1.h above. & 5.1.2.n & LOW & 1,2 \\
\hline $\begin{array}{l}\text { Modified wrench for Cf } \\
\text { package installation on } \\
\text { RR-4 }\end{array}$ & CUB 4 & Cf packages are installed in position on RR-4 using a modified in-cell wrench. & 5.2 .1 & LOW & 1,2 \\
\hline $\begin{array}{l}\text { Cf package loading } \\
\text { system leak test }\end{array}$ & CUB 4 & Previously discussed in Reference 5.1.2 above. & 5.2 .2 & $\begin{array}{l}\text { See Ref. } \\
5.1 .2\end{array}$ & See Ref. 5.1.2 \\
\hline
\end{tabular}




\begin{tabular}{|c|c|c|c|c|c|}
\hline ITEM & LOCATION & DESCRIPTION & Reference & IMPACT & COMMENTS \\
\hline $\begin{array}{l}\text { Hot feed transfer to T- } \\
408\end{array}$ & CUB 4 & Cubicle 4 vacuum onT-405 to pull hot feed into T-408. & 5.2 .3 & LOW & 1,2 \\
\hline \begin{tabular}{|l|} 
Hot feed loaded from \\
T-408 onto Cf package
\end{tabular} & CUB 4, MUA & Previously discussed in Reference 5.1.2 above. & $5.2 .4,5.2 .5$ & \begin{tabular}{|l|} 
See Ref. \\
5.1 .2 \\
\end{tabular} & See Ref. 5.1.2 \\
\hline Cf firing ward & CUB 4 & $\begin{array}{l}\text { 1/8 in. diameter stainless steel tubing with female ball joint on one end and 3/8 in. } \\
\text { compression fitting for holding the Pt capsule. }\end{array}$ & 5.3 .1 & LOW & 1,2 \\
\hline $\begin{array}{l}1 / 4 \text { in. polyethylene } \\
\text { tubing with male ball } \\
\text { joints }\end{array}$ & CUB 4 & $\begin{array}{l}\text { (2) lengths of } 1 / 4 \text { in. diameter polyethylene tubing pre-made in house with male ball joints } \\
\text { on each end. }\end{array}$ & 5.3 .2 & LOW & 1,2 \\
\hline 1 L glass bottles & CUB 4 & (2) 1 L glass bottles. & 5.3 .2 & LOW & 1,2 \\
\hline H-520 furnace & CUB 4 & Thermolyne 48010 Series furnace. & 5.3 .3 & LOW & 1,2 \\
\hline TIC-H520 & CR & $\begin{array}{l}\text { Eurotherm Model } 808 \text { programmable temperature controller located on MB } 26 \text { in the } \\
\text { Control Room. }\end{array}$ & 5.3 .4 & MED & $4,5,6$ \\
\hline
\end{tabular}


6.0 HYDROXIDE Precipitation of the Actinide Product

\begin{tabular}{|c|c|c|c|c|c|}
\hline ITEM & LOCATION & DESCRIPTION & Reference & IMPACT & COMMENTS \\
\hline $\mathrm{NaOH}$ & MUA & Stock solution of $\mathrm{NaOH}$ used for chemical make ups is typically on hand. & 6.1 .1 & LOW & 1,2 \\
\hline Nitric acid $\left(\mathrm{HNO}_{3}\right)$ & MUA & Stock solution of $\mathrm{HNO}_{3}$ used for chemical make ups is typically on hand. & 6.1 .1 & LOW & 1,2 \\
\hline Polyethylene containers & MUA, LAB & $\begin{array}{l}\text { Several sizes of polyethylene bottles/carboys are used for the chemical make ups for the } \\
\text { hydroxide precipitation operations ( } 1 \mathrm{~L}, 8 \mathrm{~L}, 25 \mathrm{~L} \text {, etc.). }\end{array}$ & 6.1 .2 & LOW & 1,2 \\
\hline Tank T-60 & TK PIT 6 & $\begin{array}{l}\text { Nominal } 63 \text { L HC shell with Ta liner evaporator tank. T-65 has an annulus filled with Hg } \\
\text { for increases heat transfer capability. }\end{array}$ & 6.2.1.a & HIGH & 11,12 \\
\hline $\begin{array}{l}\text { T-60 LL, DR, and PR } \\
\text { instr. }\end{array}$ & CR, MUA & $\begin{array}{l}\text { Foxboro pneumatic instrument is located in the CR and the DP cell and associated } \\
\text { polyethylene tubing is located in the MUA. The LL, DR, and PR lines are constructed of Ta } \\
\text { and originate in T-60 in Tank Pit 6, travel through the LAA, and are tied to the } \\
\text { polyethylene tubing that goes to the DP transmitters in the MUA. }\end{array}$ & 6.2.1.b & HIGH & $9,10,11,12$ \\
\hline T-60 sparger & $\begin{array}{l}\text { MUA, CR, TK } \\
\text { PIT } 6\end{array}$ & $\begin{array}{l}\text { There's a push button switch in CR to activate a solenoid in the MUA. An in-line orifice } \\
\text { controls air flow into the tank. }\end{array}$ & 6.2.1.c & LOW & $1,2,4$ \\
\hline $\begin{array}{l}\text { T-60 process and } \\
\text { instrument lines }\end{array}$ & TK PIT 6 & Lines are constructed of Ta and provide solution transfer routes into and out of T-60. & 6.2.1.d & HIGH & $9,10,11,12$ \\
\hline $\begin{array}{l}\text { T-60 vacuum } \\
\text { accumulator }\end{array}$ & CUB 6 & $\begin{array}{l}3 \text { in. diameter } \times 6 \text { in. long flanged glass pipe ( } \sim 1 \mathrm{~L} \text { volume) with specially fabricated } \mathrm{Zr} \text { top } \\
\text { and bottom plates. }\end{array}$ & 6.2.1.e & NCBU & \\
\hline SA-T60 needle block & CUB 6 & Specially fabricated Zr block with 2 needles for pulling samples. & 6.2.1.e & NCBU & \\
\hline $\begin{array}{l}\text { Auxiliary glass sample } \\
\text { flask }\end{array}$ & CUB 6 & $\begin{array}{l}1 \mathrm{~L} \text { glass bottle with 2-line stopper outfitted with polyethylene tubing connected to SA-T60 } \\
\text { position on CUB } 6 \text { sample rack for sampling T-60 and for connection to vacuum source. }\end{array}$ & 6.2.1.e & LOW & 1,2 \\
\hline $\begin{array}{l}\text { SA-T60 special 1-line } \\
\text { Block }\end{array}$ & CUB 6 & $\begin{array}{l}\text { Special 1-line polyethylene block with 3/8-in metal tubing on one side installed at SA-T60 } \\
\text { position on CUB } 6 \text { sample rack for sampling T-60 using auxiliary glass sample flask. }\end{array}$ & 6.2.1.e & LOW & 1,2 \\
\hline T-60 thermocouples & $\begin{array}{l}\text { CR, MUA, TK } \\
\text { PIT } 6\end{array}$ & Thermocouples, leads, and jumpers to Control Room Instruments. & 6.2.2.a & HIGH & $9,10,11,12$ \\
\hline $\begin{array}{l}\text { T-60 steam lines and } \\
\text { valves }\end{array}$ & $\begin{array}{l}\text { MUA, CR, TK } \\
\text { PIT } 6\end{array}$ & $\begin{array}{l}\text { Steam lines to the tanks, manual valves in MUA, and electrically operated solenoid valve in } \\
\text { LAA. }\end{array}$ & 6.2.2.b & HIGH & $9,10,11,12$ \\
\hline PIC-T60 & CR & Pressure controller for PCV-T60 that controls steam supply to T-60 heating/cooling jacket. & 6.2.2.c & LOW & 1,2 \\
\hline PCV-T-60 & MUA & Pressure control valve that controls steam supply to T-60 heating/cooling jacket. & 6.2.2.c & LOW & 1,2 \\
\hline $\begin{array}{l}\text { T-60 RCW lines and } \\
\text { valves }\end{array}$ & TK PIT 6, CR & Manual Valves in MUA and electrically operated solenoid valve in LAA. & 6.2.2.d & HIGH & $9,10,11,12$ \\
\hline $\mathrm{H}-60$ & CR,TK PIT 6 & Manual Valves in MUA and electrically operated solenoid valve in LAA. & 6.2.2.e & HIGH & $9,10,11,12$ \\
\hline T-60 VOG & TK PIT 6 & Condensate moves through VOG line and collects in a condensate collection tank. & 6.2.2.f & LOW & 3 \\
\hline
\end{tabular}




\begin{tabular}{|c|c|c|c|c|c|}
\hline ITEM & LOCATION & DESCRIPTION & Reference & IMPACT & COMMENTS \\
\hline Tank M-704 & MUA & \begin{tabular}{|l|l} 
Previously discussed in Reference 1.4.2 above. \\
\end{tabular} & 6.2 .3 & LOW & 3,4 \\
\hline V-T60-4 & MUA & PVC ball valve that accesses the decon line to T- 60 . & 6.2.3.a & LOW & 1,2 \\
\hline V-CU6-1 & MUA & $\begin{array}{l}\text { Solution addition line valve in MUA that goes to Cubicle } 6 \text { with a GN located in the service } \\
\text { lines on the east wall of the cubicle. }\end{array}$ & 6.2.3.b & LOW & 1,2 \\
\hline $\begin{array}{l}\text { Cubicle } 6 \text { solution } \\
\text { addition line }\end{array}$ & CUB6 & Zr goose neck located in the service lines on the east wall in Cubicle 6. & 6.2.3.c & LOW & 1,2 \\
\hline T6002 GN & CUB 6 & Standard Zr GN in left DC well. & 6.2.3.c & LOW & 1,2 \\
\hline T6007 GN & CUB 6 & Standard Zr GN in left DC well. & 6.2.4.a & & \\
\hline C4C601 GN & CUB 6 & Previously discussed in Reference 2.3.2.h above. & 6.2.4.b & LOW & 1,2 \\
\hline C4C601 jumper & $\begin{array}{l}\text { TK PIT 4, 5, } \\
\text { and } 6\end{array}$ & Previously discussed in Reference 2.3.2.i above. & 6.2.4.c & LOW & 1,2 \\
\hline C4C601 jumper & CUB 4 & Previously discussed in Reference 2.3.2.j above. & 6.2.4.d & LOW & 1,2 \\
\hline C4C601 GN & CUB 4 & Standard Zr GN on BR-4 (previously discussed in Reference 2.3.2.k above). & 6.2.4.e & LOW & 1,2 \\
\hline PS-431 position & CUB 4 & 2-line block at PS-431 position on BR-4. & 6.2.4.f & LOW & 1,2 \\
\hline FDV-440 & CUB 4 & FDV Polyethylene block located on BR-4. & 6.2.4.g & LOW & 1,2 \\
\hline Tank T-430 & CUB 4 & $\begin{array}{l}3 \text { in. diameter } \times 3 \mathrm{ft} \text { tall flanged glass column located on BR-4. T- } 430 \text { is used as a transfer } \\
\text { tank for the hydroxide precipitation operations. }\end{array}$ & 6.2.4.h & MED & $3,4,5,6$ \\
\hline HCV-T430-2 & CUB 4 & $\begin{array}{l}\text { Special } 2 \text { line polyethylene block in the HCV position with (2) 3/8 in. Zr tubes, located on } \\
\text { BR-4. }\end{array}$ & 6.2.4.i & LOW & 1,2 \\
\hline T6107 GN & CUB 6 & Standard Zr GN in Cubicle 6 left DC well. & 6.2.4.j & LOW & 1,2 \\
\hline $\begin{array}{l}\text { Cubicle } 6 \text { solution } \\
\text { addition line }\end{array}$ & CUB 6 & $\begin{array}{l}\text { Polyethylene tubing from Cubicle } 6 \text { solution addition line connected to T6107 GN in } \\
\text { Cubicle } 6 .\end{array}$ & 6.2.4.1 & & 1,2 \\
\hline Cubicle 4 vacuum & CUB 4 & Previously discussed in Reference 4.2.3.j above. & 6.2.4.m & LOW & 1,2 \\
\hline Miscellaneous supplies & $\begin{array}{l}\text { CR, MUA, } \\
\text { CUB } 4\end{array}$ & Previously discussed in Reference 1.4.1.q above. & 6.2.4.n & LOW & 1,2 \\
\hline Tank T-61 & $\begin{array}{l}\text { CR, MUA, } \\
\text { CUB 6, TK } \\
\text { PIT 6 } \\
\end{array}$ & Previously discussed in Reference 3.4.7 above. & 6.2 .5 & $\begin{array}{l}\text { See } \\
\text { Ref.3.4.7 }\end{array}$ & See Ref.3.4.7 \\
\hline Tank M-421 & MUA & $\begin{array}{l}\text { Nominal 5.5 L volume, flanged } 3 \text { in. } \times 48 \text { in. glass column tank used to deliver solutions to } \\
\text { T-54, T-43, T-45, and the LR } 4 \text { tanks. }\end{array}$ & 6.2 .6 & LOW & 3,4 \\
\hline V-M421-1 & MUA & Jamesbury ball valve, vent line to VOG. & 6.2.6.a & LOW & 1,2 \\
\hline V-M421-2 & MUA & Jamesbury ball valve, solution addition line through the funnel. & 6.2.6.b & LOW & 1,2 \\
\hline V-M421-3 & MUA & Jamesbury ball valve, 10 psig air pressure supply line. & 6.2.6.c & LOW & 1,2 \\
\hline V-M421-4 & MUA & Jamesbury ball valve, drain line/solution outlet. & 6.2.6.d & LOW & 1,2 \\
\hline V-M421-7 & MUA & Jamesbury ball valve, demineralized water addition line. & 6.2.6.e & LOW & 1,2 \\
\hline Tank M-421 & MUA & Previously discussed in Reference 6.2.6 above. & 6.2 .7 & LOW & 1,2 \\
\hline
\end{tabular}




\begin{tabular}{|c|c|c|c|c|c|}
\hline ITEM & LOCATION & DESCRIPTION & Reference & IMPACT & COMMENTS \\
\hline T6107 GN & CUB 6 & Standard Zr GN in left DC sell. & 6.2.8.a & LOW & 1,2 \\
\hline C4C601 GN & CUB 6 & Previously discussed in Reference 2.3.2.h above. & 6.2.8.b & LOW & 1,2 \\
\hline C4C601 jumper & $\begin{array}{l}\text { TK PIT 4, 5, } \\
\text { and } 6\end{array}$ & Previously discussed in Reference 2.3.2.i above. & 6.2.8.c & LOW & 1,2 \\
\hline C4C601 jumper & CUB 4 & Previously discussed in Reference 2.3.2.j above. & 6.2.8.d & LOW & 1,2 \\
\hline C4C601 GN & CUB 4 & Previously discussed in Reference 2.3.2.k above. & 6.2.8.e & LOW & 1,2 \\
\hline F-445 position & CUB 4 & $\begin{array}{l}\text { Specially fabricated Zr block [with (2) 3/8 in. diameter Zr lines] in place of the filter to } \\
\text { access the inlet to T444 on BR-4. }\end{array}$ & 6.2.8.f & LOW & 1,2 \\
\hline Tank T-444 & CUB 4 & $\begin{array}{l}3 \text { in. diameter } \times 3 \mathrm{ft} \text { tall flanged glass column located on LR } 4 . \mathrm{T}-444 \text { is used as a transfer } \\
\text { tank for the hydroxide precipitation operations. }\end{array}$ & 6.2.8.g & MED & $3,4,5,6$ \\
\hline HCV-T444-2 & CUB 4 & $\begin{array}{l}\text { Special } 2 \text { line polyethylene block in the HCV position with (2) 3/8 in. Zr tubes, located on } \\
\text { LR } 4 .\end{array}$ & 6.2.8.h & LOW & 1,2 \\
\hline Deep bed filter & CUB 4 & Specially fabricated filter with stainless steel sintered metal filters & 6.2.8.i & LOW & 1,2 \\
\hline PS-431 position & CUB 4 & $\begin{array}{l}\text { Specially fabricated Zr block [with (2) 3/8 in. diameter Zr lines] in place of a phase } \\
\text { separator to access the inlet and outlet of PS-431 position on BR-4. }\end{array}$ & 6.2.8.j & LOW & 1,2 \\
\hline FDV-440 & CUB 4 & Previously discussed in Reference 6.2.4.g above. & 6.2.8.k & LOW & 1,2 \\
\hline Tank T-430 & CUB 4 & Previously discussed in Reference 6.2.4.h above. & 6.2.8.1 & MED & $3,4,5,6$ \\
\hline HCV-T430-2 & CUB 4 & $\begin{array}{l}\text { Special } 2 \text { line polyethylene block in the HCV position with (2) 3/8 in. Zr tubes, located on } \\
\text { BR-4. }\end{array}$ & 6.2.8.m & LOW & 1,2 \\
\hline T2003 GN & CUB 6 & Standard Zr GN in Cubicle 6 DC well (T2003 line is teed to P71004 line). & 6.2.8.n & LOW & 1,2 \\
\hline HCV-T430-1 & CUB 4 & $\begin{array}{l}\text { Special } 2 \text { line polyethylene block in the HCV position with (2) 3/8 in. Zr tubes, located on } \\
\text { BR-4. }\end{array}$ & 6.2.8.0 & LOW & 1,2 \\
\hline 7PDV-P710-2 & CUB 7 & $\begin{array}{l}\text { 7PDV located on BR-7 with Type B polyethylene block (set to } 2 \text { to block P71004 line teed } \\
\text { to T2003 line to T-20). }\end{array}$ & 6.2.8.p & LOW & 1,2 \\
\hline Cubicle 4 vacuum & CUB 4 & Previously discussed in Reference 4.2.3.j above. & 6.2.8.q & LOW & 1,2 \\
\hline Miscellaneous supplies & $\begin{array}{l}\text { CR, MUA, } \\
\text { CUB } 4\end{array}$ & Previously discussed in Reference 1.4.1.q above. & 6.2.8.r & LOW & 1,2 \\
\hline Tank T-20 & TK PIT 2 & Nominal 74 L tank constructed of HC located in Tank Pit 2. & 6.2.9.a & HIGH & 11,12 \\
\hline $\begin{array}{l}\text { T-20 LL, DR, and PR } \\
\text { instr. }\end{array}$ & CR, MUA & $\begin{array}{l}\text { Foxboro pneumatic instrument is located in the CR and the DP cell and associated } \\
\text { polyethylene tubing is located in the MUA. The LL, DR, and PR lines are constructed of Ta } \\
\text { and originate in T-20 in Tank Pit 2, travel through the LAA, and are tied to the } \\
\text { polyethylene tubing that goes to the DP transmitters in the MUA. }\end{array}$ & 6.2.9.b & HIGH & $9,10,11,12$ \\
\hline T-20 sparger & $\begin{array}{l}\text { MUA, CR, TK } \\
\text { PIT } 2\end{array}$ & $\begin{array}{l}\text { There's a push button switch in CR to activate a solenoid in the MUA. An in-line orifice } \\
\text { controls air flow into the tank. }\end{array}$ & 6.2.9.c & LOW & $1,2,4$ \\
\hline $\begin{array}{l}\text { T-20 process and } \\
\text { instrument lines }\end{array}$ & TK PIT 2 & Lines are constructed of $\mathrm{Zr}$ and provide solution transfer routes into and out of T-20. & 6.2.9.d & HIGH & $9,10,11,12$ \\
\hline $\begin{array}{l}\text { T-20 vacuum } \\
\text { accumulator }\end{array}$ & CUB 6 & $\begin{array}{l}3 \text { in. diameter } \times 6 \text { in. long flanged glass pipe ( } \sim 1 \mathrm{~L} \text { volume) with specially fabricated } \mathrm{Zr} \text { top } \\
\text { and bottom plates. }\end{array}$ & 6.2.9.e & NCBU & NCBU \\
\hline
\end{tabular}




\begin{tabular}{|c|c|c|c|c|c|}
\hline ITEM & LOCATION & DESCRIPTION & Reference & IMPACT & COMMENTS \\
\hline SA-T20 needle block & CUB 6 & Specially fabricated Zr block with 2 needles for pulling samples. & 6.2.9.e & NCBU & NCBU \\
\hline $\begin{array}{l}\text { Auxiliary glass sample } \\
\text { flask }\end{array}$ & CUB 6 & $\begin{array}{l}1 \text { L glass bottle with 2-line stopper outfitted with polyethylene tubing connected to SA-T20 } \\
\text { position on CUB } 6 \text { sample rack for sampling T-20 and for connection to vacuum source. }\end{array}$ & 6.2.9.e & LOW & 1,2 \\
\hline $\begin{array}{l}\text { SA-T20 special 1-line } \\
\text { block }\end{array}$ & CUB 6 & $\begin{array}{l}\text { Special 1-line polyethylene block with 3/8 in. metal tubing on one side installed at SA-T20 } \\
\text { position on CUB } 6 \text { sample rack for sampling T-20 using auxiliary glass sample flask. }\end{array}$ & 6.2.9.e & LOW & 1,2 \\
\hline Tank M-421 & MUA & Previously discussed in Reference 6.2.6 above. & 6.2 .10 & LOW & 1,2 \\
\hline Tank T-43 & TK PIT 4 & $\begin{array}{l}\text { Precipitate is dissolved and product is collected in T-43. Applicable transfer route } \\
\text { equipment and set up previously discussed in Reference 6.2.6 and 6.28. Previously } \\
\text { discussed in Reference } 2.3 .11 \text { and } \mathbf{2 . 3 . 1 2} \text { above. }\end{array}$ & 6.2 .11 & $\begin{array}{l}\text { See Ref. } \\
2.3 .13 \\
2,3,14\end{array}$ & $\begin{array}{l}\text { See Ref. } \\
2.3 .13,2,3,14\end{array}$ \\
\hline Tank M-421 & MUA & Previously discussed in Reference 6.2.6 above. & 6.2 .12 & LOW & 1,2 \\
\hline T-43 sampler & CUB 4 & Previously discussed in Reference 2.3.13.e above. & 6.2 .13 & LOW & 1,2 \\
\hline T-20 sampler & CUB 6 & Previously discussed in Reference 6.2.9.e above. & 6.2 .14 & LOW & 1,2 \\
\hline Tank M-704 & MUA & Previously discussed in Reference 1.4.2 above. & 6.2 .15 & LOW & 1,2 \\
\hline T-61 sampler & CUB 6 & Previously discussed in Reference 3.4.7.e above. & 6.2 .15 & LOW & 1,2 \\
\hline Tank M-704 & MUA & Previously discussed in Reference 1.4.2 above. & 6.2 .16 & LOW & 1,2 \\
\hline Tank T-60 & $\begin{array}{l}\text { TK PIT 6, } \\
\text { CUB } 6\end{array}$ & Previously discussed in Reference 6.2.1 and 6.2.2 above. & 6.2 .16 & \begin{tabular}{|l|} 
See Ref. \\
$6.2 .1-2$ \\
\end{tabular} & $\begin{array}{l}\text { See Ref. } \\
6.2 .1-2 \\
\end{array}$ \\
\hline
\end{tabular}


7.0 OXALATE Precipitation Operations

\begin{tabular}{|c|c|c|c|c|c|}
\hline ITEM & LOCATION & DESCRIPTION & Reference & IMPACT & COMMENTS \\
\hline Oxalic acid, nitric acid & MUA & A supply of oxalic acid and $\mathrm{HNO}_{3}$ is typically kept on hand for chemical make ups. & $\begin{array}{l}\text { 7.1.1.a, } \\
\text { 7.1.1.b, } \\
\text { 7.1.1.c }\end{array}$ & LOW & 1,2 \\
\hline Polyethylene containers & MUA, LAB & $\begin{array}{l}\text { Several sizes of polyethylene bottles/carboys are used for the chemical make ups for the } \\
\text { hydroxide precipitation operations }(1 \mathrm{~L}, 8 \mathrm{~L}, 25 \mathrm{~L} \text {, etc.). }\end{array}$ & $\begin{array}{l}\text { 7.1.1.a, } \\
\text { 7.1.1.b, } \\
\text { 7.1.1.c }\end{array}$ & LOW & 1,2 \\
\hline Tank T-54 & TK PIT 5 & $\begin{array}{l}\text { Nominal } 22 \text { L HC shell with Ta liner evaporator tank. T-54 has an annulus filled with Hg } \\
\text { for increases heat transfer capability. }\end{array}$ & 7.2.1.a & HIGH & 11,12 \\
\hline $\begin{array}{l}\text { T-54 LL, DR, and PR } \\
\text { instr. }\end{array}$ & CR, MUA & $\begin{array}{l}\text { Foxboro pneumatic instrument is located in the CR and the DP cell and associated } \\
\text { polyethylene tubing is located in the MUA. The LL, DR, and PR lines are constructed of Ta } \\
\text { and originate in T-54 in Tank Pit 5, travel through the LAA, and are tied to the } \\
\text { polyethylene tubing that goes to the DP transmitters in the MUA. }\end{array}$ & 7.2.1.b & HIGH & $9,10,11,12$ \\
\hline T-54 sparger & $\begin{array}{l}\text { MUA, CR, TK } \\
\text { PIT } 5\end{array}$ & $\begin{array}{l}\text { There's a push button switch in CR to activate a solenoid in the MUA. An in-line orifice } \\
\text { controls air flow into the tank. }\end{array}$ & 7.2.1c & LOW & $1,2.4$ \\
\hline $\begin{array}{l}\text { T-54 process and } \\
\text { instrument lines }\end{array}$ & TK PIT 5 & Lines are constructed of Ta and provide solution transfer routes into and out of T-54. & 7.2.1.d & HIGH & $9,10,11,12$ \\
\hline $\begin{array}{l}\text { T-54 vacuum } \\
\text { accumulator }\end{array}$ & CUB 4 & $\begin{array}{l}3 \text { in. diameter } \times 6 \text { in. long flanged glass pipe ( } \sim 1 \mathrm{~L} \text { volume) with specially fabricated } \mathrm{Zr} \text { top } \\
\text { and bottom plates. }\end{array}$ & 7.2.1.e & NCBU & \\
\hline SA-T54 needle block & CUB 4 & Specially fabricated Zr block with two needles for pulling samples. & 7.2.1.e & NCBU & \\
\hline $\begin{array}{l}\text { Auxiliary glass sample } \\
\text { flask }\end{array}$ & CUB 4 & $\begin{array}{l}1 \text { L glass bottle with 2-line stopper outfitted with polyethylene tubing connected to SA-T54 } \\
\text { position on CUB } 4 \text { sample rack for sampling T-54 and for connection to vacuum source. }\end{array}$ & 7.2.1.e & LOW & 1,2 \\
\hline $\begin{array}{l}\text { SA-T54 special 1-line } \\
\text { block }\end{array}$ & CUB 4 & $\begin{array}{l}\text { Special 1-line polyethylene block with 3/8 in. metal tubing on one side installed at SA-T54 } \\
\text { position on CUB } 4 \text { sample rack for sampling T-54 using auxiliary glass sample flask. }\end{array}$ & 7.2.1.e & LOW & 1,2 \\
\hline T-54 thermocouples & $\begin{array}{l}\text { CR, MUA, TK } \\
\text { PIT } 5\end{array}$ & Thermocouples, leads, and jumpers to Control Room Instruments. & 7.2.2.a & HIGH & $9,10,11,12$ \\
\hline $\begin{array}{l}\text { T-54 steam lines and } \\
\text { valves }\end{array}$ & $\begin{array}{l}\text { MUA, CR, TK } \\
\text { PIT 5 }\end{array}$ & $\begin{array}{l}\text { Steam lines to the tanks, manual valves in MUA, and electrically operated solenoid valve in } \\
\text { LAA. }\end{array}$ & 7.2.2.b & HIGH & $9,10,11,12$ \\
\hline PIC-T54 & CR & Pressure controller for PCV-54 that controls steam supply to T-54 heating/cooling jacket. & 7.2.2.c & LOW & 1,2 \\
\hline
\end{tabular}




\begin{tabular}{|c|c|c|c|c|c|}
\hline ITEM & LOCATION & DESCRIPTION & Reference & IMPACT & COMMENTS \\
\hline PCV-T-54 & MUA & Pressure control valve that controls steam supply to T-54 heating/cooling jacket. & 7.2.2.c & LOW & 1,2 \\
\hline $\begin{array}{l}\text { T-54 RCW lines and } \\
\text { valves }\end{array}$ & CR, TK PIT 5 & Manual Valves in MUA and electrically operated solenoid valve in LAA. & 7.2.2.d & HIGH & $9,10,11,12$ \\
\hline $\mathrm{H}-54$ & CR,TK PIT 5 & Manual Valves in MUA and electrically operated solenoid valve in LAA. & 7.2.2.e & HIGH & 9,10,11,12 \\
\hline T-54 VOG & TK PIT 5 & Condensate moves through VOG line and collects in a condensate collection tank. & 7.2.2.f & LOW & 3 \\
\hline Tank M-421 & MUA & Previously discussed in Reference 6.2.6 above. & 7.2 .3 & LOW & $1,2,3,4$ \\
\hline Tank M-450 & MUA & $\begin{array}{l}\text { Nominal 5.5 L volume, flanged } 3 \text { in. } \times 48 \text { in. glass column tank used to deliver solutions to } \\
\text { T-54, T-43, T-45, and the LR } 4 \text { tanks. }\end{array}$ & 7.2.4 & LOW & $1,2,3,4$ \\
\hline V-M450-1 & MUA & Jamesbury ball valve, vent line to VOG. & 7.2.4.a & LOW & 1,2 \\
\hline V-M450-2 & MUA & Jamesbury ball valve, solution addition line through the funnel. & 7.2.4.b & LOW & 1,2 \\
\hline V-M450-3 & MUA & Jamesbury ball valve, 5 psig air pressure supply line. & 7.2.4.c & LOW & 1,2 \\
\hline V-M450-4 & MUA & Jamesbury ball valve, drain line/solution outlet. & 7.2.4.d & LOW & 1,2 \\
\hline V-M450-7 & MUA & Jamesbury ball valve, demineralized water addition line. & 7.2.4.e & LOW & 1,2 \\
\hline $\begin{array}{l}\text { P-460 heating loop } \\
\text { pump }\end{array}$ & MUA, CR & $\begin{array}{l}\text { Centrifugal type water pump located in the MUA. There is also an ON/OFF switch in the } \\
\text { CR. }\end{array}$ & 7.2.5.a & MED & 5,6 \\
\hline V-P460-1 & MUA & Gate valve, pump discharge to heater inlet. & 7.2.5.b & LOW & 1,2 \\
\hline V-P460-2 & MUA & Gate valve, heater discharge. & 7.2.5.b & LOW & 1,2 \\
\hline V-P460-5 & MUA & Gate valve, return loop water to P-69. & 7.2.5.b & LOW & 1,2 \\
\hline V-P460-6 & MUA & Gate valve, from RCW supply. & 7.2.5.b & LOW & 1,2 \\
\hline V-P460-7 & MUA & Gate valve, to RCW return. & 7.2.5.b & LOW & 1,2 \\
\hline V-CAL260-1 & MUA & Ball valve, outlet from RCW supply to FI-CAL260. & 7.2.5.b & LOW & 1,2 \\
\hline V-CAL260-2 & MUA & Ball valve, outlet from FI-CAL260 to calorimeter in Cubicle 2. & 7.2.5.b & LOW & 1,2 \\
\hline V-CAL260-3 & MUA & Ball valve, outlet from Calorimeter in Cubicle 2 to RCW Return. & 7.2.5.b & LOW & 1,2 \\
\hline V-CAL260-4 & MUA & Ball valve, outlet from H-460 heater to V-CAL260-5. & 7.2.5.b & LOW & 1,2 \\
\hline V-CAL260-5 & MUA & Ball valve, outlet V-CAL260-4 to H-460. & 7.2.5.b & LOW & 1,2 \\
\hline V-CAL260-6 & MUA & Ball valve, outlet from Calorimeter to V-CAL260-7. & 7.2.5.b & LOW & 1,2 \\
\hline V-CAL260-7 & MUA & Ball valve, outlet to RCW Return. & 7.2.5.b & LOW & 1,2 \\
\hline PRV-P460-3 & MUA & Pressure relief valve for the P-460 heating/cooling water loop. & 7.2.5.b & LOW & 1,2 \\
\hline H-460 heater & MUA & Electric heaters for heating the water in the P-460 heating/cooling water loop. & 7.2.5.c & MED & 5,6 \\
\hline
\end{tabular}




\begin{tabular}{|c|c|c|c|c|c|}
\hline ITEM & LOCATION & DESCRIPTION & Reference & IMPACT & COMMENTS \\
\hline TC-H460 & CR & $\begin{array}{l}\text { Temperature Recorder and Controller located in the CR controls amount of cooling water } \\
\text { allowed into the loop for controlling the heat up and cool down of T-451. }\end{array}$ & 7.2.5.d & MED & 5,6 \\
\hline FI-P460 & MUA & Flow indicator for the P-460 heating/cooling water loop. & 7.2.5.e & LOW & 1,2 \\
\hline H-460 thermocouples & MUA & Type K thermocouples measuring the temperature of the P-460 heating/cooling water loop. & 7.2.5.f & LOW & 1,2 \\
\hline T5407 GN & CUB 4 & $\begin{array}{l}\text { Standard Zr GN in Cubicle } 4 \text { DC well with polyethylene line connected to line on special } \\
\text { FDV at FDV-440 position on BR-4. }\end{array}$ & 7.3.1.a & LOW & 1,2 \\
\hline FDV-440 & CUB 4 & $\begin{array}{l}\text { Special polyethylene block for FDV with 3/8 in. ZR tube installed at blank position on } \\
\text { polyethylene block located on BR-4. }\end{array}$ & 7.3.1.b & LOW & 1,2 \\
\hline Tank T-430 & CUB 4 & Previously discussed in Reference 6.2.4.h above. & 7.3.1.c & LOW & $1,2,4$ \\
\hline HCV-T430-1 & CUB 4 & Special HCV type PVC toggle valve. & 7.3.1.d & LOW & 1,2 \\
\hline F-432 position & CUB 4 & Standard filter element in specially fabricate filter assembly located on BR-4. & 7.3.1.e & LOW & 1,2 \\
\hline HCV-F432-2 & CUB 4 & Special HCV type PVC toggle valve. & 7.3.1.f & LOW & 1,2 \\
\hline F43202 GN & CUB 4 & $\begin{array}{l}\text { Standard Zr GN on BR-4 in Cubicle } 4 \text { with polyethylene line connected to T45105 GN on } \\
\text { LR-4. }\end{array}$ & 7.3.1.g & LOW & 1,2 \\
\hline 7PDV-T451-1 & CUB 4 & Type D polyethylene block located on LR-4. & 7.3.1.h & LOW & 1,2 \\
\hline HCV-T451-1 & CUB 4 & Special HCV type PVC toggle valve. & 7.3.1.i & LOW & 1,2 \\
\hline $\begin{array}{l}\text { Tank T-451 and T-451 } \\
\text { agitator }\end{array}$ & CUB 4 & $\begin{array}{l}\text { Specially modified nominal } 5 \text { L glass precipitator tank with agitator for mixing located on } \\
\text { LR } 4 \text {. }\end{array}$ & 7.3.1.j & HIGH & $9,10,12$ \\
\hline Tank T-456 & CR, CUB 4 & $\begin{array}{l}\text { Nominal } 18 \mathrm{~L} \text { volume } \mathrm{Zr} \text { collection tank located on LR } 4 \text { used to collect raffinate and } \\
\text { recycle product. }\end{array}$ & 7.3.2 & HIGH & $9,10,12$ \\
\hline Tank T-457 & CR, CUB 4 & $\begin{array}{l}\text { Nominal } 18 \mathrm{~L} \text { volume } \mathrm{Zr} \text { collection tank located on LR } 4 \text { used to collect raffinate and } \\
\text { recycle product. }\end{array}$ & 7.3 .2 & HIGH & $9,10,12$ \\
\hline Tank T-458 & CR, CUB 4 & $\begin{array}{l}\text { Nominal } 18 \mathrm{~L} \text { volume Zr collection tank located on LR } 4 \text { used to collect raffinate and } \\
\text { recycle product. }\end{array}$ & 7.3.2 & HIGH & $9,10,12$ \\
\hline Tank T-459 & CR, CUB 4 & $\begin{array}{l}\text { Nominal } 10 \mathrm{~L} \text { volume Zr tank located on LR } 4 \text { used as a knock tank for T-456, T457, and } \\
\text { T458. }\end{array}$ & 7.3 .2 & HIGH & $9,10,12$ \\
\hline P-460 heating loop & CR, CUB 4 & Previously discussed in Reference 7.2.5 above. & 7.3.3.a & \begin{tabular}{|l|} 
See Ref. \\
7.2 .5 \\
\end{tabular} & See Ref. 7.2 .5 \\
\hline T-451 agitator & CR, CUB 4 & Previously discussed in Reference 7.3.1. $\mathbf{j}$ above. & 7.3.3.b, d & \begin{tabular}{|l|} 
See Ref. \\
7.3.1.j \\
\end{tabular} & See Ref. 7.3.1.j \\
\hline Tank M-450 & MUA & Previously discussed in Reference 7.3.2 above. & 7.3.3.c & LOW & 1,2 \\
\hline Tank T-456 & CR, CUB 4 & Previously discussed in Reference 7.3.2 above. & 7.3.3.e & \begin{tabular}{|l|} 
See Ref. \\
7.3 .2 \\
\end{tabular} & See Ref. 7.3.2 \\
\hline $\begin{array}{l}\text { Tank T-451 and T-451 } \\
\text { agitator }\end{array}$ & CUB 4 & Previously discussed in Reference 7.3.1.j above. & 7.3.4.a & \begin{tabular}{|l|} 
See Ref. \\
7.3.1.j \\
\end{tabular} & See Ref. 7.3.1.j \\
\hline
\end{tabular}




\begin{tabular}{|c|c|c|c|c|c|}
\hline ITEM & LOCATION & DESCRIPTION & Reference & IMPACT & COMMENTS \\
\hline HV-T451-2 position & CUB 4 & Special 1-line polyethylene block installed in HV position. & 7.3.4.b & LOW & 1,2 \\
\hline 7PDV-T451-2 position & CUB 4 & Special 1-line polyethylene block installed at the 7PDV position. & 7.3.4.c & LOW & 1,2 \\
\hline F-454 Filter & CUB 4 & Metal filter housing with sintered metal filters and manual stirrer located on LR 4. & 7.3.4.d & HIGH & 9,10 \\
\hline FDV-F454 & CUB 4 & Polyethylene block FDV located on LR 4. & 7.3.4.e & LOW & 1,2 \\
\hline HCV-P452-1 & CUB 4 & $\begin{array}{l}\text { Special } 2 \text { line polyethylene block in the HCV position with (2) 3/8 in. Zr tubes, located on } \\
\text { LR } 4 .\end{array}$ & 7.3.4.f & LOW & 1,2 \\
\hline $\mathrm{P}-452$ & CUB 4 & $\begin{array}{l}\text { Specially designed and fabricated vacuum/pressure diaphragm pump (TRU pump) located } \\
\text { on LR } 4 \text { for use with for the oxalate precipitation operations. }\end{array}$ & 7.3.4.g & MED & $5,6,8$ \\
\hline FDV-P452 & CUB 4 & Polyethylene block FDV located on LR 4. & 7.3.4.h & LOW & 1,2 \\
\hline HCV-T456 & CUB 4 & Special HCV type PVC toggle valve located on LR 4. & 7.3.4.i & LOW & 1,2 \\
\hline HCV-T457 & CUB 4 & Special HCV type PVC toggle valve located on LR 4. & 7.3.4.j & LOW & 1,2 \\
\hline HCV-T458 & CUB 4 & Special HCV type PVC toggle valve located on LR 4. & 7.3.4.k & LOW & 1,2 \\
\hline Tank T-456 & CR, CUB 4 & Previously discussed in Reference 7.3.2 above. & 7.3.4.1 & HIGH & $9,10,12$ \\
\hline Tank M-450 & MUA & Previously discussed in Reference 7.2.4 above. & 7.3.4.m & LOW & $1,2,3,4$ \\
\hline $\begin{array}{l}\text { Tank T-451 and T-451 } \\
\text { agitator }\end{array}$ & CUB 4 & Previously discussed in Reference 7.3.1.j above. & 7.3.5.a & HIGH & $9,10,12$ \\
\hline Tank M-450 & MUA & Previously discussed in Reference 7.2.4 above. & 7.3.5.a & LOW & $1,2,3,4$ \\
\hline $\begin{array}{l}\text { T-451 to T-458 transfer } \\
\text { route }\end{array}$ & CR, CUB 4 & Previously discussed in Reference 7.3.4.a-k above. & 7.3.5.c & $\begin{array}{l}\text { See Ref. } \\
\text { 7.2.4.a-j }\end{array}$ & \begin{tabular}{|l|} 
See Ref. \\
7.2.4.a-j \\
\end{tabular} \\
\hline Tank T-458 & CR, CUB 4 & Previously discussed in Reference 7.3.2 above. & 7.3.5.c & HIGH & $9,10,12$ \\
\hline 7PDV-T451-1 & CUB 4 & Previously discussed in Reference 7.3.1.h above. & 7.3.6.a & LOW & 1,2 \\
\hline $\begin{array}{l}\text { Tank T-451 and T-451 } \\
\text { agitator }\end{array}$ & CUB 4 & Previously discussed in Reference 7.3.1.j above. & 7.3.6.a & HIGH & $9,10,12$ \\
\hline Tank T-458 and T-451 & CR, CUB 4 & Previously discussed in Reference 7.3.1.j and 7.3.2 above. & 7.3.6.a & HIGH & $9,10,12$ \\
\hline Tank M-450 & MUA & Previously discussed in Reference 7.2.4 above. & 7.3.6.b & LOW & $1,2,3,4$ \\
\hline $\begin{array}{l}\text { P-460 heating loop } \\
\text { system }\end{array}$ & $\begin{array}{l}\text { MUA, CR, } \\
\text { CUB 4 } \\
\end{array}$ & Previously discussed in Reference 7.2.5 above. & 7.3.7.a & \begin{tabular}{|l|} 
See Ref. \\
7.2 .5 \\
\end{tabular} & See Ref. 7.2 .5 \\
\hline Tank M-450 & MUA & Previously discussed in Reference 7.2.4 above. & 7.3.7.b & LOW & $1,2,3,4$ \\
\hline $\begin{array}{l}\text { Tank T-451 and T-451 } \\
\text { Agitator }\end{array}$ & CR, CUB 4 & Previously discussed in Reference 7.3.1.j above. & 7.3.7.c, d & HIGH & $9,10,11,12$ \\
\hline Tank M-450 & MUA & Previously discussed in Reference 7.2.4 above. & 7.3.7.e & LOW & $1,2,3,4$ \\
\hline $\begin{array}{l}\text { P-460 heating loop } \\
\text { system }\end{array}$ & $\begin{array}{l}\text { MUA, CR, } \\
\text { CUB 4 }\end{array}$ & Previously discussed in Reference 7.2.5 above. & 7.3.7.f & $\begin{array}{l}\text { See Ref. } \\
7.2 .5\end{array}$ & See Ref. 7.2 .5 \\
\hline Tank M-450 & MUA & Previously discussed in Reference 7.2.4 above. & 7.3.7.g & LOW & $1,2,3,4$ \\
\hline
\end{tabular}




\begin{tabular}{|c|c|c|c|c|c|}
\hline ITEM & LOCATION & DESCRIPTION & Reference & IMPACT & COMMENTS \\
\hline $\begin{array}{l}\text { Tank T-451 and T-451 } \\
\text { agitator }\end{array}$ & CR, CUB 4 & Previously discussed in Reference 7.3.1.j above. & 7.3.7.h & HIGH & $9,10,11,12$ \\
\hline $\begin{array}{l}\text { P-460 heating loop } \\
\text { system }\end{array}$ & $\begin{array}{l}\text { MUA, CR, } \\
\text { CUB } 4 \\
\end{array}$ & Previously discussed in Reference 7.2.5 above. & 7.3.7.i & \begin{tabular}{|l|} 
See Ref. \\
7.2 .5 \\
\end{tabular} & See Ref. 7.2 .5 \\
\hline Tank T-458 & CR, CUB 4 & Previously discussed in Reference 7.3.2 above. & 7.3.7.i & HIGH & $9,10,11,12$ \\
\hline $\begin{array}{l}\text { T-451 to T-458 transfer } \\
\text { route }\end{array}$ & CR, CUB 4 & Previously discussed in Reference 7.3.4.a-k above. & 7.3.8.a & \begin{tabular}{|l|} 
See Ref. \\
7.2.4. $\mathrm{a}-\mathrm{j}$ \\
\end{tabular} & \begin{tabular}{|l} 
See Ref. \\
7.2.4. $\mathrm{a}-\mathrm{j}$ \\
\end{tabular} \\
\hline Tank T-458 & CR, CUB 4 & Previously discussed in Reference 7.3.2 above. & 7.3.8.b & HIGH & $9,10,11,12$ \\
\hline Tank M-450 & MUA & Previously discussed in Reference 7.2.4 above. & 7.3.9.a & LOW & $1,2,3,4$ \\
\hline $\begin{array}{l}\text { Tank T-451 and T-451 } \\
\text { agitator }\end{array}$ & CUB 4 & Previously discussed in Reference 7.3.1.j above. & 7.3.9.a & HIGH & $9,10,11,12$ \\
\hline $\begin{array}{l}\text { T- } 451 \text { to } \mathrm{T}-43 \text { transfer } \\
\text { route }\end{array}$ & CR, CUB 4 & Previously discussed in Reference 7.3.4.a-k above. & 7.3.9.c & \begin{tabular}{|l|} 
See Ref. \\
7.2.4. $\mathrm{a}-\mathrm{j}$ \\
\end{tabular} & \begin{tabular}{|l|} 
See Ref. \\
7.2.4. $\mathrm{a}-\mathrm{j}$ \\
\end{tabular} \\
\hline Tank T-43 & CUB 4 & Previously discussed in Reference 2.3.11 and 2.3.12 above. & $\begin{array}{l}\text { 7.3.11.a, } \\
\text { 7.3.11.b }\end{array}$ & \begin{tabular}{|l|} 
See Ref. \\
$2.3 .11-12$ \\
\end{tabular} & $\begin{array}{l}\text { See Ref. } \\
2.3 .11-12\end{array}$ \\
\hline
\end{tabular}


8.0 Curium OXIDE Operations

\begin{tabular}{|c|c|c|c|c|c|}
\hline ITEM & LOCATION & DESCRIPTION & Reference & IMPACT & COMMENTS \\
\hline Tank T-43 evaporation & TK PIT 4, CR & Previously discussed in References 2.3.11 and 2.3.12 above. & 8.1.1, 8.1.2 & $\begin{array}{l}\text { See Ref. } \\
2.3 .11 \\
2.3 .12 \\
\end{array}$ & \begin{tabular}{|l|} 
See Ref. \\
2.3.11, 2.3.12
\end{tabular} \\
\hline Tank M-421 & MUA & Previously discussed in Reference 6.2.6 above. & 8.1 .3 & LOW & $1,2,3.4$ \\
\hline Tank T-43 & TK PIT 4, CR & Previously discussed in References 2.3.11 and 2.3.12 above. & 8.1 .4 & \begin{tabular}{|l|} 
See Ref. \\
2.3.11, \\
2.3 .12 \\
\end{tabular} & $\begin{array}{l}\text { See Ref. } \\
\text { 2.3.11, 2.3.12 }\end{array}$ \\
\hline Tank T-43 sampling & CUB 4 & Previously discussed in Reference 2.3.11.e above. & 8.1 .5 & LOW & $1,2,13$ \\
\hline Quartz column & CUB 4 & Special quartz column for installation at C-448 position on LR 4 is fabricated in house. & 8.2 .1 & MED & 5 \\
\hline Polyethylene bottles & CUB 4, MUA & $1 \mathrm{~L}$ and $250-\mathrm{ml}$ polyethylene bottles for use loading resin into the quartz column. & 8.2 .2 & LOW & 1 \\
\hline C-448 column & CUB 4 & $\begin{array}{l}\text { Position on LR } 4 \text { where the quartz column for loading the Cm solution onto resin. See } \\
\text { Reference 8.2.1 above for discussion of the quartz column. }\end{array}$ & 8.2.3.a & MED & 5 \\
\hline P44601 GN & CUB 4 & $\begin{array}{l}\text { Standard Zr GN located on LR-4 outfitted with a JACO elbow and a special male ball joint } \\
\text { elbow. }\end{array}$ & 8.2.3.b & LOW & 1,2 \\
\hline HCV-P446-1 & CUB 4 & Special pass through polyethylene block in the HCV position, located on LR 4. & 8.2.3.c & LOW & 1,2 \\
\hline $\mathrm{P}-446$ & CUB 4 & $\begin{array}{l}\text { Specially designed and fabricated vacuum/pressure diaphragm pump (TRU pump) located } \\
\text { on LR } 4 \text { for use with for the resin column loading operations. }\end{array}$ & 8.2.3.d & MED & $5,6,8$ \\
\hline RE-P446 position & CUB 4 & $\begin{array}{l}\text { Specially fabricated pass through polyethylene block installed instead of a radiation } \\
\text { element. }\end{array}$ & 8.2.3.e & LOW & 1,2 \\
\hline \begin{tabular}{|l|} 
HCV-P446-2 \\
\end{tabular} & CUB 4 & Special HCV type PVC toggle valve. & 8.2.3.f & LOW & 1,2 \\
\hline Tank T-43 & TK PIT 4, CR & Previously discussed in References 2.3.11 and 2.3.12 above. & 8.2.3.g & $\begin{array}{l}\text { See Ref. } \\
2.3 .11 \\
2.3 .12 \\
\end{array}$ & $\begin{array}{l}\text { See Ref. } \\
2.3 .11,2.3 .12\end{array}$ \\
\hline DOWEX 50-X8 resin & MUA & Supply of characterized resin with a mesh size of $60-80 \mu \mathrm{m}$. & 8.2 .4 & LOW & 1 \\
\hline Tank T-45 & TK PIT 4, CR & Column loading raffinate tanks (See Reference 8.2.6 below for discussion of T-45). & 8.2 .5 & \begin{tabular}{|l|} 
See Ref. \\
8.2 .6$,
\end{tabular} & See Ref. 8.2.6, \\
\hline Tank T-43 & TK PIT 4, CR & Previously discussed in References 2.3.11 and 2.3.12 above. & 8.2 .5 & $\begin{array}{l}\text { See Ref. } \\
2.3 .11 \\
2.3 .12\end{array}$ & $\begin{array}{l}\text { See Ref. } \\
2.3 .11,2.3 .12\end{array}$ \\
\hline Tank T-45 & TK PIT 4 & Nominal 24.7 L Zr tank with Zr probes. & 8.2.6.a & HIGH & 11,12 \\
\hline $\begin{array}{l}\text { T-45 LL, DR, and PR } \\
\text { instr. }\end{array}$ & CR, MUA & $\begin{array}{l}\text { Foxboro pneumatic instrument is located in the CR and the DP cell and associated } \\
\text { polyethylene tubing is located in the MUA. The LL, DR, and PR lines are constructed of Ta } \\
\text { and originate in T-60 in Tank Pit } 6 \text {, travel through the LAA, and are tied to the } \\
\text { polyethylene tubing that goes to the DP transmitters in the MUA. }\end{array}$ & 8.2.6.b & HIGH & $9,10,11,12$ \\
\hline
\end{tabular}




\begin{tabular}{|c|c|c|c|c|c|}
\hline ITEM & LOCATION & DESCRIPTION & Reference & IMPACT & COMMENTS \\
\hline T-45 sparger & $\begin{array}{l}\text { MUA, CR, TK } \\
\text { PIT } 4\end{array}$ & $\begin{array}{l}\text { There's a push button switch in CR to activate a solenoid in the MUA. An in-line orifice } \\
\text { controls air flow into the tank. }\end{array}$ & 8.2.6.c & LOW & $1,2,4$ \\
\hline \begin{tabular}{|l|} 
T-45 process and \\
instrument lines
\end{tabular} & TK PIT 4 & Lines are constructed of Ta and provide solution transfer routes into and out of T-45. & 8.2.6.d & HIGH & $9,10,11,12$ \\
\hline \begin{tabular}{|l|} 
T-45 vacuum \\
accumulator
\end{tabular} & CUB 4 & $\begin{array}{l}3 \text { in. diameter } \times 6 \text { in. long flanged glass pipe ( } \sim 1 \mathrm{~L} \text { volume) with specially fabricated } \mathrm{Zr} \text { top } \\
\text { and bottom plates. }\end{array}$ & 8.2.6.e & NCBU & N/A \\
\hline SA-T45 needle block & CUB 4 & Specially fabricated Zr block with two needles for pulling samples. & 8.2.6.e & NCBU & N/A \\
\hline $\begin{array}{l}\text { Auxiliary glass sample } \\
\text { flask }\end{array}$ & CUB 4 & $\begin{array}{l}1 \text { L glass bottle with 2-line stopper outfitted with polyethylene tubing connected to SA-T45 } \\
\text { position on CUB } 4 \text { sample rack for sampling T- } 45 \text { and for connection to vacuum source. }\end{array}$ & 8.2.6.e & LOW & 1,2 \\
\hline $\begin{array}{l}\begin{array}{l}\text { SA-T45 special 1-line } \\
\text { block }\end{array} \\
\end{array}$ & CUB 4 & $\begin{array}{l}\text { Special 1-line polyethylene block with } 3 / 8 \text { in. metal tubing on one side installed at SA-T45 } \\
\text { position on CUB } 4 \text { sample rack for sampling T- } 45 \text { using auxiliary glass sample flask. }\end{array}$ & 8.2.6.e & LOW & 1,2 \\
\hline FDV-435 & CUB 4 & Polyethylene block FDV located on BR-4. & 8.3.1.a & LOW & 1,2 \\
\hline \begin{tabular}{|l|} 
FDV-T444-1 \\
\end{tabular} & CUB 4 & Polyethylene block FDV located on LR 4. & 8.3.1.b & LOW & 1,2 \\
\hline F-445 position & CUB 4 & $\begin{array}{l}\text { Special pass through polyethylene block or special 2-line polyethylene block with 3/8 in. } \\
\text { polyethylene line and PVC ball valve in line. }\end{array}$ & 8.3.1.c & LOW & 1,2 \\
\hline \begin{tabular}{|l} 
Tank T-444 \\
\end{tabular} & CUB 4 & Previously discussed in Reference 6.2.8.g above. & 8.3.1.d & MED & $3,4,5,6$ \\
\hline \begin{tabular}{|l|l} 
HCV-T444-2 \\
\end{tabular} & CUB 4 & Special HCV type PVC toggle valve. & 8.3.2.a & LOW & 1,2 \\
\hline FDV-T444-3 & CUB 4 & Polyethylene block FDV located on LR-4. & 8.3.2.b & LOW & 1,2 \\
\hline C-448 column & CUB 4 & See Reference 8.2.3.a above for discussion of the quartz column. & 8.3.2.c & MED & 5 \\
\hline HCV-P446-1 & CUB 4 & Special HCV type PVC toggle valve on LR-4. & 8.3.2.d & LOW & 1,2 \\
\hline \begin{tabular}{|l|l|l|l}
$\mathrm{P}-446$ \\
\end{tabular} & CUB 4 & Previously discussed in Reference 8.2.3.c above. & 8.3.2.e & MED & $5,6,8$ \\
\hline RE-P446 position & CUB 4 & $\begin{array}{l}\text { Specially fabricated pass through polyethylene block installed instead of a radiation } \\
\text { element. }\end{array}$ & 8.3.2.f & LOW & 1,2 \\
\hline HCV-P446-2 & CUB 4 & $\begin{array}{l}\text { Special HCV type PVC toggle valve on LR-4. Previously discussed in Reference 8.2.3.f } \\
\text { above. }\end{array}$ & 8.3.2.g & & \\
\hline Tank T-43 & TK PIT 4, CR & Previously discussed in References 2.3 .11 and 2.3 .12 above. & 8.3.2.h & \begin{tabular}{|l} 
See Ref. \\
2.3 .11 , \\
2.3 .12
\end{tabular} & \begin{tabular}{|l|} 
See Ref. \\
2.3.11, 2.3.12
\end{tabular} \\
\hline \begin{tabular}{|l|l} 
Tank T-45 \\
\end{tabular} & TK PIT 4, CR & Previously discussed in Reference 8.2.6 above. & 8.3.2.i & \begin{tabular}{|l|} 
See Ref. \\
8.2 .6 \\
\end{tabular} & See Ref. 8.2.6 \\
\hline Tank M-421 & MUA & Previously discussed in Reference 6.2.6 above. & 8.3.2.j & LOW & 1,2 \\
\hline \begin{tabular}{|l|} 
FDV-T444-2 \\
\end{tabular} & CUB 4 & Polyethylene block FDV located on LR-4. & 8.3.2.k & LOW & 1,2 \\
\hline HCV-T444-1 & CUB 4 & Special HCV type PVC toggle valve on LR-4. & 8.3.2.1 & LOW & 1,2 \\
\hline Cubicle 4 vacuum & CUB 4 & Previously discussed in Reference 4.2.3.j above. & 8.3.2.m & LOW & LOW 1,2 \\
\hline Miscellaneous supplies & $\begin{array}{l}\text { CR, MUA, } \\
\text { CUB } 4\end{array}$ & Previously discussed in Reference 1.4.1.q above. & 8.3.2.n & LOW & 1,2 \\
\hline
\end{tabular}




\begin{tabular}{|c|c|c|c|c|c|}
\hline ITEM & LOCATION & DESCRIPTION & Reference & IMPACT & COMMENTS \\
\hline C-448 flushing & CUB 4 & $\begin{array}{l}\text { Water flush of T- } 444 \text { to C- } 448 \text { along with removal of stopper from C-448 and flushing C- } \\
448 \text { with in-cell demineralized water. Reference 8.3.1 and 8.3.2 above for route set up. }\end{array}$ & $\begin{array}{l}\text { 8.3.3, 8.3.4, } \\
\text { and 8.3.5 }\end{array}$ & LOW & 1,2 \\
\hline $\mathrm{P}-446$ & CUB 4 & Previously discussed in Reference 8.3.2.e above. & 8.3 .6 & MED & $5,6,8$ \\
\hline T-45 sampling & $\begin{array}{l}\text { TK PIT 4, } \\
\text { CUB } 4\end{array}$ & Previously discussed in Reference 8.2.6.e above. & 8.3 .7 & LOW & 1,2 \\
\hline T-43 evaporation & $\begin{array}{l}\text { TK PIT 4, } \\
\text { MUA, CR }\end{array}$ & Previously discussed in References 2.3.11 and 2.3.12 above. & 8.3 .8 & $\begin{array}{l}\text { See Ref. } \\
2.3 .11 \text {, } \\
2.3 .12\end{array}$ & $\begin{array}{l}\text { See Ref. } \\
\text { 2.3.11, 2.3.12 }\end{array}$ \\
\hline H-419 furnace & CUB 4, CR & $\begin{array}{l}\text { Specially fabricated "round" furnace located on LR } 4 \text { for firing resin while in the quartz } \\
\text { column. }\end{array}$ & 8.4.1.a & HIGH & $9,10,11$ \\
\hline Quartz column top & CUB 4 & Specially fitted quartz top for the quartz column equipped with a frit. & 8.4.1.b & MED & 4 \\
\hline H-419 lid & CUB 4 & Lid for H-419 is fabricated in house to fit onto H-419. & 8.4.1.c & LOW & 1,2 \\
\hline $\begin{array}{l}1 / 4 \text { in. diameter } \\
\text { polyethylene tubing }\end{array}$ & CUB 4 & $\begin{array}{l}\text { Length of } 1 / 4 \text { in. diam. Polyethylene tubing with male ball joint on one end and the other end } \\
\text { connected to GN at R } 7 \text { position in Cubicle } 4 \text { for purge of C-448 during firing. }\end{array}$ & 8.4.1.d & LOW & 1,2 \\
\hline TIC-H419 controller & CR & $\begin{array}{l}\text { Thermolyne Model } 808 \text { programmable temperature controller located on MB } 27 \text { in the } \\
\text { Control Room. }\end{array}$ & 8.4.1.e & MED & 5,6 \\
\hline Ar- $\mathrm{H}_{2}$ purge & CUB 4, MUA & Supply cylinders of Ar-H2 gas are located in RCW room in MUA. & 8.4.1.f & LOW & 1,2 \\
\hline Pt crucible & CUB 4 & $\begin{array}{l}\text { Specially fabricated Pt lined Inconel crucible made by an outside vendor. Process may no } \\
\text { longer exist for fabrication of these. }\end{array}$ & 8.4.2.a & HIGH & 9,10 \\
\hline Quartz column & CUB 4 & Previously discussed in Reference 8.2.3.a above. & 8.4.2.b & MED & 5 \\
\hline H-520 muffle furnace & CUB 4 & Previously discussed in Reference 5.3.3 above. & 8.4.2.c & LOW & 1,2 \\
\hline Pt crucible holder & CUB 4 & $\begin{array}{l}\text { Specially fabricated Inconel holder for handling the Pt crucible and placing into the muffle } \\
\text { furnace. }\end{array}$ & 8.4.2.c & MED & 5,6 \\
\hline \begin{tabular}{|l|}
$1 / 4$ in. diameter \\
polyethylene tubing
\end{tabular} & CUB 4 & $\begin{array}{l}\text { Length of } 1 / 4 \text { in. diam. polyethylene tubing with male ball joint on one end and the other end } \\
\text { connected to Cubicle } 4 \text { air supply. }\end{array}$ & 8.4.2.d & LOW & 1,2 \\
\hline $\begin{array}{l}\text { Pt crucible furnace } \\
\text { holder }\end{array}$ & CUB 4 & Previously discussed in Reference 8.4.2.c above. & 8.4.2.e & MED & 5,6 \\
\hline TIC-H520 controller & CR & $\begin{array}{l}\text { Eurotherm Model } 808 \text { programmable temperature controller located on MB } 26 \text { in the } \\
\text { Control Room. Previously discussed in Reference 5.3.4 above. }\end{array}$ & 8.4.2.f & MED & 5,6 \\
\hline Stainless steel tray & CUB 4 & $\begin{array}{l}\text { Stainless steel tray (photo tray) sometimes with master slave manipulators channels } \\
\text { installed for ease of handling in the hot cell cubicles. }\end{array}$ & 8.5.1.a & LOW & 1,2 \\
\hline $\begin{array}{l}\text { Certified/calibrated } \\
\text { balance }\end{array}$ & CUB 4 & $\begin{array}{l}\text { Certified/calibrated balance with appropriate weight range for weighing curium oxide in its } \\
\text { storage container. }\end{array}$ & 8.5.1.b & LOW & 1,2 \\
\hline $\begin{array}{l}\text { Certified standard } \\
\text { weights }\end{array}$ & CUB 4 & Typical weights for use during balance calibration verification. & 8.5.1.c & LOW & 1,2 \\
\hline $\begin{array}{l}\begin{array}{l}\text { Stainless steel screen } \\
\text { assembly }\end{array} \\
\end{array}$ & CUB 4 & $\begin{array}{l}\text { Stainless steel } 210 \mu \mathrm{m} \text { mesh size screen, bottom pan, top, and specially fabricated weight } \\
\text { for screening curium oxide. }\end{array}$ & 8.5.1.d & MED & 5,6 \\
\hline $\begin{array}{l}\text { Aluminum weighing } \\
\text { dish }\end{array}$ & CUB 4 & Small Al weighing dish for weighing curium oxide. & 8.5.1.e & LOW & \begin{tabular}{|l}
1,2 \\
\end{tabular} \\
\hline
\end{tabular}




\begin{tabular}{|l|l|l|l|l|l|}
\hline \multicolumn{1}{|c|}{ ITEM } & LOCATION & \multicolumn{1}{|c|}{ DESCRIPTION } & Reference & IMPACT & COMMENTS \\
\hline $\begin{array}{l}\text { Small glass powder } \\
\text { funnel }\end{array}$ & CUB 4 & Small glass powder funnel with wide spout for pouring curium oxide. & $8.5 .1 . f$ & LOW & 1,2 \\
\hline $\begin{array}{l}10 \mathrm{ml} \text { glass graduated } \\
\text { cylinder }\end{array}$ & CUB 4 & $\begin{array}{l}\text { Standard 10 ml glass graduated cylinder used for determining the tap density of curium } \\
\text { oxide. }\end{array}$ & $8.5 .1 . \mathrm{g}$ & LOW & 1,2 \\
\hline $250 \mathrm{ml}$ bottle holder & CUB 4 & $\begin{array}{l}\text { Specially fabricated bottle holder for 250 ml polyethylene bottles used to stabilize a 10 ml } \\
\text { glass graduated cylinder during tap density determination of curium oxide. }\end{array}$ & $8.5 .1 . \mathrm{h}$ & LOW & 1,2 \\
\hline $\begin{array}{l}\text { Stainless steel storage } \\
\text { container }\end{array}$ & CUB 4 & $\begin{array}{l}\text { Specially fabricated stainless steel containers with locking lids used for curium oxide } \\
\text { storage. }\end{array}$ & $8.5 .1 . \mathrm{i}$ & LOW & 1,2 \\
\hline $\begin{array}{l}\text { Glass sample vial with } \\
\text { lid }\end{array}$ & CUB 4 & $\begin{array}{l}\text { Small glass sample vial used to obtain a sample of curium oxide to submit to Chemical } \\
\text { Science Division for analysis. }\end{array}$ & $8.5 .1 . \mathrm{j}$ & LOW & 1,2 \\
\hline
\end{tabular}


9.0 Miscellaneous Process Support Systems and Equipment

\begin{tabular}{|c|c|c|c|c|c|}
\hline ITEM & LOCATION & DESCRIPTION & Reference & IMPACT & COMMENTS \\
\hline $\begin{array}{l}\text { Tank L-131 VOG } \\
\text { scrubber }\end{array}$ & TK PIT 7 & $\begin{array}{l}\text { Nominal } 192 \mathrm{~L} \text { tank with a HC bottom section and stainless steel top section used to scrub } \\
\text { the VOG to remove acid vapors. L-131 has an upper section filled with packing to provide } \\
\text { good liquid-gas contact for scrubbing. }\end{array}$ & 9.1.1.a & HIGH & $9,10,11,12$ \\
\hline $\begin{array}{l}\text { L-131 LL and DR } \\
\text { instrumentation }\end{array}$ & CR, MUA & $\begin{array}{l}\text { Foxboro pneumatic instrument is located in the CR and the DP cell and associated } \\
\text { polyethylene tubing is located in the MUA. The LL and DR lines are constructed of } \\
\text { stainless steel and originate in L-131 in Tank Pit 7, travel through the LAA, and are tied to } \\
\text { the polyethylene tubing that goes to the DP transmitters in the MUA. }\end{array}$ & 9.1.1.b & HIGH & $9,10,11,12$ \\
\hline $\begin{array}{l}\mathrm{L}-131 \mathrm{PR} \text { and PdR } \\
\text { instrumentation }\end{array}$ & CR, MUA & $\begin{array}{l}\text { Yokogawa pneumatic instrument ( } 10 \text { years old) is located in the CR and the DP cell and } \\
\text { associated polyethylene tubing is located in the MUA. This instrument is equipped with a } \\
\text { digital readout as well as a pen recorder. The PR and PdR lines are constructed of stainless } \\
\text { steel and originate in L- } 131 \text { in Tank Pit } 7 \text {, travel through the LAA, and are tied to the } \\
\text { polyethylene tubing that goes to the DP transmitters in the MUA. }\end{array}$ & 9.1.1.c & HIGH & $9,10,11,12$ \\
\hline $\begin{array}{l}\text { L-131 probe flush } \\
\text { down station }\end{array}$ & LAA & $\begin{array}{l}\text { An access station to the LL, DR, PR, PdR, probes that consists of isolation valves and quick } \\
\text { disconnects for periodic flush down of the probes to clear line plugs and restrictions. The } \\
\text { station is provided with a demineralize water supply line with a quick disconnect. }\end{array}$ & 9.1.1.d & LOW & 1,2 \\
\hline $\begin{array}{l}\mathrm{J}-131 \mathrm{~A} \text { and } \mathrm{J}-131 \mathrm{~B} \\
\text { recirculation pumps }\end{array}$ & $\begin{array}{l}\text { LAA (pipe } \\
\text { tunnel) }\end{array}$ & $\begin{array}{l}\text { The pumps recirculation the scrubbing liquid from the bottom section of L-131 to above the } \\
\text { packing. }\end{array}$ & 9.1.1.e & HIGH & $9,10,12$ \\
\hline L-131 process lines & TK PIT 7 & $\begin{array}{l}\text { Lines are constructed of stainless steel and provide solution transfer routes into and out of } \\
\text { L-131. }\end{array}$ & 9.1.1.f & HIGH & $9,10,11,12$ \\
\hline $\begin{array}{l}\text { L-131 liquid level } \\
\text { controller }\end{array}$ & CR, MUA & $\begin{array}{l}\text { LC-131, L-131 liquid level control instrument, maintains a constant level in L-131 by } \\
\text { adding demineralize water to the tank from the MUA. The controller is located on MB-1 in } \\
\text { the Control Room with the addition valve located in the MUA. }\end{array}$ & 9.1.1.g & MED & $4,5,6$ \\
\hline SA-L131 needle block & CUB 7 & Specially fabricated Zr block with two needles for pulling samples. & 9.1.1.h & NCBU & N/A \\
\hline $\begin{array}{l}\text { Auxiliary glass sample } \\
\text { flask }\end{array}$ & CUB 7 & $\begin{array}{l}1 \text { L glass bottle with 2-line stopper outfitted with polyethylene tubing connected to SA- } \\
\text { L131 position on CUB } 7 \text { sample rack for sampling L-131 and for connection to vacuum } \\
\text { source. }\end{array}$ & 9.1.1.h & LOW & 1,2 \\
\hline
\end{tabular}




\begin{tabular}{|c|c|c|c|c|c|}
\hline ITEM & LOCATION & DESCRIPTION & Reference & IMPACT & COMMENTS \\
\hline $\begin{array}{l}\text { SA-L131 special 1-line } \\
\text { block }\end{array}$ & CUB 7 & $\begin{array}{l}\text { Special 1-line polyethylene block with 3/8 in. metal tubing on one side installed at SA- } \\
\text { L131 position on CUB } 7 \text { sample rack for sampling L-131 using auxiliary glass sample } \\
\text { flask. }\end{array}$ & 9.1.1.h & LOW & 1,2 \\
\hline Tank M-5 & MUA & $\begin{array}{l}\begin{array}{l}\text { Nominal } 140 \text { L volume, stainless steel tank used to deliver solutions (typically } \mathrm{NaOH} \text { ) to } \\
\text { the L-131 Scrubber. }\end{array} \\
\end{array}$ & 9.1 .2 & LOW & 1,2 \\
\hline V-M5-1 & MUA & Jamesbury ball valve, vent line to VOG. & 9.1.2.a & LOW & 1,2 \\
\hline V-M5-2 & MUA & Jamesbury ball valve, solution addition line through the funnel. & 9.1.2.b & LOW & 1,2 \\
\hline V-M5-3 & MUA & Jamesbury ball valve, 10 psig air pressure supply line. & 9.1.2.c & LOW & 1,2 \\
\hline V-M5-4 & MUA & Jamesbury ball valve, drain line/solution outlet. & 9.1.2.d & LOW & 1,2 \\
\hline V-M5-7 & MUA & Jamesbury ball valve, sparger for M-5 (10 psig air pressure supply). & 9.1.2.e & LOW & 1,2 \\
\hline V-M5-8 & MUA & Ball valve, outlet to L-131 (through LCV-L131). & 9.1.2.f & LOW & 1,2 \\
\hline V-M5-9 & MUA & Ball valve, 10 psig air supply line (tied to V-M5-1 valve to vent). & 9.1.2.g & LOW & 1,2 \\
\hline V-M5-13 & MUA & Ball valve, outlet line. & 9.1.2.h & LOW & 1,2 \\
\hline Tank M-500 & MUA & $\begin{array}{l}\text { Nominal } 15.2 \mathrm{~L} \text { volume, flanged } 4 \text { in. } \times 72 \text { in. glass column tank used as a vacuum/pressure } \\
\text { transfer tank to deliver caustic and demineralize water to } \mathrm{M}-5 \text { and } \mathrm{M}-40 \text { in the MUA. }\end{array}$ & 9.1 .3 & MED & $3,5,6$ \\
\hline V-M500-1 & MUA & Jamesbury ball valve, vent line to VOG. & 9.1.3.a & LOW & 1,2 \\
\hline V-M500-2 & MUA & Jamesbury ball valve, vacuum supply. & 9.1.3.b & LOW & 1,2 \\
\hline V-M500-3 & MUA & Jamesbury ball valve, 5 psig air pressure supply line. & 9.1.3.c & LOW & 1,2 \\
\hline V-M500-4 & MUA & Jamesbury ball valve, drain line/solution outlet. & 9.1.3.d & LOW & 1,2 \\
\hline V-M500-5 & MUA & Jamesbury ball valve, outlet to M-40 (V-M40-5 and V-M500-7). & 9.1.3.e & LOW & 1,2 \\
\hline V-M500-6 & MUA & Ball valve, outlet. & 9.1.3.f & LOW & 1,2 \\
\hline V-M500-7 & MUA & Ball valve, inlet into M-5 from V-M500-5. & 9.1.3.g & LOW & 1,2 \\
\hline V-M500-8 & MUA & Ball valve, auxiliary inlet into $\mathrm{M}-500$. & 9.1.3.h & LOW & 1,2 \\
\hline V-F500-1 & MUA & Ball valve, inlet from the drum room caustic header to F-500. & 9.1.3.i & LOW & 1,2 \\
\hline V-F500-2 & MUA & Ball valve, inlet into M-500 from caustic header (bypass of M-500). & 9.1.3.j & LOW & 1,2 \\
\hline \begin{tabular}{|l|} 
V-F500-3 \\
\end{tabular} & MUA & Ball valve, drain valve for F-500. & 9.1.3.j. & \begin{tabular}{|l|l|l} 
LOW \\
\end{tabular} & \begin{tabular}{|l|l}
, 2 \\
1,2
\end{tabular} \\
\hline V-F500-5 & MUA & Ball valve, inlet line from $\mathrm{KOH}$ line into caustic header to F-500. & 9.1.3.1 & LOW & 1,2 \\
\hline V-F500-6 & MUA & Ball valve, inlet line from $\mathrm{KOH}$ line into caustic header to F-500. & 9.1.3.m & LOW & \begin{tabular}{|l|l|l}
1,2 \\
1,2
\end{tabular} \\
\hline V-F500-7 & MUA & Ball valve, inlet into M-500 from $\mathrm{NaOH}$ line (bypass of $\mathrm{M}-500$ ). & 9.1.3.n & LOW & 1,2 \\
\hline V-F500-8 & MUA & Ball valve, outlet from F-500 to M-500. & 9.1.3.0 & LOW & 1,2 \\
\hline L-131 sampler & CUB 7 & Previously discussed in Reference 9.1.1.h above. & 9.1.4.a & LOW & $\mid 1,2$ \\
\hline \begin{tabular}{|l|l} 
HIC-J131 \\
\end{tabular} & CR & Manual operator that supplies air pressure to HCV-J131A valve. & 9.1.4.b & LOW & 1,2 \\
\hline HCV-J131A & $\begin{array}{l}\text { LAA (pipe } \\
\text { tunnel) }\end{array}$ & L-131 Scrubber drain valve to F-126. & 9.1.4.b & HIGH & $9,10,112$ \\
\hline
\end{tabular}




\begin{tabular}{|c|c|c|c|c|c|}
\hline ITEM & LOCATION & DESCRIPTION & Reference & IMPACT & COMMENTS \\
\hline Tank M-5 & MUA & Previously discussed in Reference 9.1.2 above. & 9.1.4.c, d & $\begin{array}{l}\text { See Ref. } \\
9.1 .2\end{array}$ & See Ref. 9.1.2 \\
\hline Tank M-500 & MUA & Previously discussed in Reference 9.1.3 above. & 9.1.4.c, d & \begin{tabular}{|l|} 
See Ref. \\
9.1 .3 \\
\end{tabular} & See Ref. 9.1.3 \\
\hline Tank F-115 & $\begin{array}{l}\text { WASTE TK } \\
\text { PT }\end{array}$ & Nominal 1892 L tank constructed of HC with Zr probes. & 9.2.1.a & HIGH & 11,12 \\
\hline $\begin{array}{l}\text { F-115 LL, DR, and PR } \\
\text { instr. }\end{array}$ & CR, MUA & $\begin{array}{l}\text { Yokogawa instrument is located in the CR and the DP cell and associated polyethylene } \\
\text { tubing is located in the MUA. The LL, DR, and PR lines are constructed of } \mathrm{Zr} \text { and originate } \\
\text { in F-115 in the waste tank pit, travel through the LAA, and are tied to the polyethylene } \\
\text { tubing that goes to the DP transmitters in the MUA. }\end{array}$ & 9.2.1.b & HIGH & $9,10,11,12$ \\
\hline F-115 sparger & $\begin{array}{l}\text { MUA, CR, } \\
\text { WASTE TK } \\
\text { PT }\end{array}$ & $\begin{array}{l}\text { There's an electric toggle switch in CR to activate a solenoid in the MUA which in turns } \\
\text { opens HCV-F115-6. An in-line orifice controls air flow into the tank. }\end{array}$ & 9.2.1.c & LOW & $1,2,4$ \\
\hline $\begin{array}{l}\text { F-115 process and } \\
\text { instrument lines }\end{array}$ & $\begin{array}{l}\text { WASTE TK } \\
\text { PT }\end{array}$ & Lines are constructed of Zr and provide solution transfer routes into and out of F-115. & 9.2.1.d & HIGH & $9,10,11,12$ \\
\hline \begin{tabular}{|l|} 
F-115 vacuum \\
accumulator
\end{tabular} & CUB 7 & $\begin{array}{l}3 \text { in. diameter } \times 6 \text { in. long flanged glass pipe ( } \sim 1 \text { L volume) with specially fabricated } \mathrm{Zr} \text { top } \\
\text { and bottom plates. }\end{array}$ & 9.2.1.e & NCBU & N/A \\
\hline SA-F115 needle block & CUB 7 & Specially fabricated Zr block with 2 needles for pulling samples. & 9.2.1.e & NCBU & N/A \\
\hline $\begin{array}{l}\text { Auxiliary glass sample } \\
\text { flask }\end{array}$ & CUB 7 & $\begin{array}{l}1 \text { L glass bottle with 2-line stopper outfitted with polyethylene tubing connected to SA- } \\
\text { F115 position on CUB } 7 \text { sample rack for sampling F-115 and for connection to vacuum } \\
\text { source. }\end{array}$ & 9.2.1.e & LOW & 1,2 \\
\hline $\begin{array}{l}\text { SA-F115 special 1-line } \\
\text { block }\end{array}$ & CUB 7 & $\begin{array}{l}\text { Special 1-line polyethylene block with 3/8 in. metal tubing on one side installed at SA-F115 } \\
\text { position on CUB } 7 \text { sample rack for sampling F-115 using auxiliary glass sample flask. }\end{array}$ & 9.2.1.e & LOW & 1,2 \\
\hline F-115 sample & CUB 7 & Previously discussed in Reference 9.2.1.e above. & 9.2.2.a & LOW & 1,2 \\
\hline J-F115 & $\begin{array}{l}\text { WASTE TK } \\
\text { PT, MUA, CR, }\end{array}$ & Steam jet used to transfer F-115 contents to F-126. & 9.2.2.b & MED & 4,12 \\
\hline F-115 sample & CUB 7 & Previously discussed in Reference 9.2.1.e above. & 9.2.3.a & LOW & 1,2 \\
\hline J-F115 & $\begin{array}{l}\text { WASTE TK } \\
\text { PT, MUA, CR, }\end{array}$ & Previously discussed in Reference 9.2.2.c above. & 9.2.3.c & MED & 4,12 \\
\hline
\end{tabular}




\begin{tabular}{|c|c|c|c|c|c|}
\hline ITEM & LOCATION & DESCRIPTION & Reference & IMPACT & COMMENTS \\
\hline $\begin{array}{l}\text { SA-F115 special 1-line } \\
\text { block }\end{array}$ & CUB 7 & $\begin{array}{l}\text { Special 1-line polyethylene block with 3/8 in. metal tubing on one side installed at SA-F115 } \\
\text { position on CUB } 7 \text { sample rack used for transferring material out of or in to F-115. }\end{array}$ & 9.2.4.a & LOW & 1,2 \\
\hline T73402 GN & CUB 7 & $\begin{array}{l}\text { Standard Ta GN on RR-7, inlet for T-734 with polyethylene tubing connected to 1-line } \\
\text { block at SA-F115 position Reference 1.6.1.d above. }\end{array}$ & 9.2.4.b & LOW & 1,2 \\
\hline PS-733 & CUB 7 & Previously discussed in Reference 2.3.2.c above. & 9.2.4.c & LOW & 1,2 \\
\hline HV-T734-2 & CUB 7 & Previously discussed in Reference 1.6.1.f above. & 9.2.4.d & LOW & 1,2 \\
\hline Tank T-734 & CUB 7 & Previously discussed in Reference 1.6.1.g above. & 9.2.4.e & MED & 3,4 \\
\hline HV-T734-3 & CUB 7 & Previously discussed in Reference 1.6.1.h above. & 9.2.4.f & LOW & 1,2 \\
\hline HV-T734-1 & CUB 7 & Previously discussed in Reference 1.6.1.k above. & 9.2.4.g & LOW & 1,2 \\
\hline T73404 GN & CUB 7 & $\begin{array}{l}\text { Gooseneck on RR-7, outlet for T-734 with polyethylene tubing connected to T7703 GN. } \\
\text { Previously discussed in Reference 1.6.1.i above. }\end{array}$ & 9.2.4.h & LOW & 1,2 \\
\hline T7703 GN & CUB 7 & Standard Zr GN in Cubicle 7 right DC well. & 9.2.4.i & LOW & 1,2 \\
\hline T-77 & TK PT 7 & Discussed in Reference 9.2.5 below. & 9.2.4.j & LOW & 1,2 \\
\hline KP-735 & CUB 7 & Previously discussed in Reference 1.6.1.I above. & 9.2.4.k & LOW & 1,2 \\
\hline CU7AJ & CUB 7 & Previously discussed in Reference 1.4.1.p above. & 9.2.4.1 & LOW & 1,2 \\
\hline Miscellaneous supplies & $\begin{array}{l}\text { CR, MUA, } \\
\text { CUB } 7\end{array}$ & Previously discussed in Reference 1.4.1.q above. & 9.2.4.m & LOW & 1,2 \\
\hline Tank T-77 & TK PIT 7 & $\sim 295 \mathrm{~L}$ tank constructed of HC with HC lines. & 9.2.5.a & HIGH & 11,12 \\
\hline $\begin{array}{l}\text { T-77 LL, DR, and PR } \\
\text { instr. }\end{array}$ & $\begin{array}{l}\text { CR, MUA, } \\
\text { CUB } 7\end{array}$ & $\begin{array}{l}\text { Foxboro pneumatic instrument is located in the CR and the DP cell and associated } \\
\text { polyethylene tubing is located in the MUA. The LL, DR, and PR lines are constructed of Ta } \\
\text { and originate in T-77 in Tank Pit 7, travel through the LAA, and are tied to the } \\
\text { polyethylene tubing that goes to the DP transmitters in the MUA. }\end{array}$ & 9.2.5.b & HIGH & $9,10,11,12$ \\
\hline T-77 sparger & $\begin{array}{l}\text { MUA, CR, TK } \\
\text { PIT } 7\end{array}$ & $\begin{array}{l}\text { There's a push button switch in CR to activate a solenoid in the MUA. An in-line orifice } \\
\text { controls air flow into the tank. }\end{array}$ & 9.2.5.c & LOW & $1,2,4$ \\
\hline $\begin{array}{l}\text { T-77 process and } \\
\text { instrument lines }\end{array}$ & TK PIT 7 & Lines are constructed of HC. & 9.2.5.d & HIGH & $9,10,11,12$ \\
\hline $\begin{array}{l}\text { T-77 vacuum } \\
\text { accumulator }\end{array}$ & CUB 7 & $\begin{array}{l}3 \text { in. diameter } \times 6 \text { in. long flanged glass pipe ( } \sim 1 \mathrm{~L} \text { volume) with specially fabricated } \mathrm{Zr} \text { top } \\
\text { and bottom plates. }\end{array}$ & 9.2.5.e & NCBU & N/A \\
\hline SA-T77 needle block & CUB 7 & Specially fabricated Zr block with two needles for pulling samples. & 9.2.5.e & NCBU & N/A \\
\hline $\begin{array}{l}\text { Auxiliary glass sample } \\
\text { flask }\end{array}$ & CUB 7 & $\begin{array}{l}1 \text { L glass bottle with 2-line stopper outfitted with polyethylene tubing connected to SA-T77 } \\
\text { position on CUB } 6 \text { sample rack for sampling T-77 and for connection to vacuum source. }\end{array}$ & 9.2.5.e & LOW & 1,2 \\
\hline
\end{tabular}




\begin{tabular}{|c|c|c|c|c|c|}
\hline ITEM & LOCATION & DESCRIPTION & Reference & IMPACT & COMMENTS \\
\hline $\begin{array}{l}\text { SA-T77 special 1-line } \\
\text { block }\end{array}$ & CUB 7 & $\begin{array}{l}\text { Special 1-line polyethylene block with } 3 / 8 \text { in. metal tubing on one side installed at SA-T77 } \\
\text { position on CUB } 7 \text { sample rack for sampling T-77 using auxiliary glass sample flask. }\end{array}$ & 9.2.5.e & LOW & 1,2 \\
\hline T7707 GN & CUB 7 & $\begin{array}{l}\text { Standard Zr GN in Cubicle } 7 \text { right DC well with polyethylene line connected to T73402 } \\
\text { GN on RR-7. }\end{array}$ & 9.2.6.a & LOW & 1,2 \\
\hline T73402 GN & CUB 7 & $\begin{array}{l}\text { Standard Ta GN on RR-7, inlet for T-734 with polyethylene tubing connected to T7707 } \\
\text { GN. Reference 1.6.1.d above. }\end{array}$ & 9.2.6.b & LOW & 1,2 \\
\hline PS-733 & CUB 7 & Previously discussed in Reference 2.3.2.c above. & 9.2.6.C & LOW & 1,2 \\
\hline HV-T734-2 & CUB 7 & Previously discussed in Reference 1.6.1.f above. & 9.2.6.d & LOW & 1,2 \\
\hline Tank T-734 & CUB 7 & Previously discussed in Reference 1.6.1.g above. & 9.2.6.e & MED & 3,4 \\
\hline HV-T734-3 & CUB 7 & Previously discussed in Reference 1.6.1.h above. & 9.2.6.f & LOW & 1,2 \\
\hline HV-T734-1 & CUB 7 & Previously discussed in Reference 1.6.1.k above. & 9.2.6.g & LOW & 1,2 \\
\hline T73404 GN & CUB 7 & $\begin{array}{l}\text { Standard Ta GN on RR-7, outlet for T-734 with polyethylene tubing connected to 1-line } \\
\text { block at SA-F115 position. Previously discussed in Reference 1.6.1.i above. }\end{array}$ & 9.2.6.h & LOW & 1,2 \\
\hline $\begin{array}{l}\text { SA-F115 special 1-line } \\
\text { block }\end{array}$ & CUB 7 & Previously discussed in Reference 9.2.4.a above. & 9.2.6.i & LOW & 1,2 \\
\hline KP-735 & CUB 7 & Previously discussed in Reference 1.6.1.I above. & 9.2.6.j & LOW & 1,2 \\
\hline CU7AJ & CUB 7 & Previously discussed in Reference 1.4.1.p above. & 9.2.6.k & LOW & 1,2 \\
\hline Miscellaneous supplies & $\begin{array}{l}\text { CR, MUA, } \\
\text { CUB } 6\end{array}$ & Previously discussed in Reference 1.4.1.q above. & 9.2.6.1 & LOW & 1,2 \\
\hline Tanks M-1 /M-2 & MUA & Previously discussed in References 1.2.1 and 1.2.2 above. & 9.2.7.a & LOW & 3,4 \\
\hline M-70 & MUA & Previously discussed in Reference 2.1.4 above. & 9.2.7.b & LOW & 3,4 \\
\hline T-77 sampler & CUB 7 & Previously discussed in Reference 9.2.5.e above. & 9.2.7.c, d & LOW & 1,2 \\
\hline T7707 GN & CUB 7 & $\begin{array}{l}\text { Standard Zr GN in Cubicle } 7 \text { right DC well with polyethylene line connected to T60702 } \\
\text { GN on BR-6. Previously discussed in Reference 9.2.6.a above. }\end{array}$ & 9.2.8.a & LOW & 1,2 \\
\hline T760702 GN & CUB 6 & Standard Zr GN on BR-6 (inlet line to PS-607). & 9.2.8.b & LOW & 1,2 \\
\hline PCV ball valve & CUB 6 & Connected to T60702 GN and polyethylene line from T7707 GN. & 9.2.8.c & LOW & 1,2 \\
\hline PS-607 & CUB 6 & $\begin{array}{l}\text { Phase separator is } 3 \text { in. diameter } \times 6 \text { in. long flanged glass pipe ( } \sim 1 \mathrm{~L} \text { volume) with } \\
\text { specially fabricated } \mathrm{Zr} \text { top and bottom plates installed on BR-6. }\end{array}$ & 9.2.8.d & LOW & 1,2 \\
\hline T-607 & CUB 6 & $\begin{array}{l}3 \text { in. diameter } \times 3 \mathrm{ft} \text { tall flanged glass column located on BR64. T-607 is used as a transfer } \\
\text { tank for CLEANEX and other miscellaneous solutions. }\end{array}$ & 9.2.8.e & MED & $3,4,5,6$ \\
\hline HV-607 & CUB 6 & Polyethylene pass through block installed at HV-607 position. & 9.2.8.f & LOW & 1,2 \\
\hline T60703 GN & CUB 6 & Standard Zr GN on BR-6 (outlet line from T-607). & 9.2.8.g & LOW & 1,2 \\
\hline PCV ball valve & CUB 6 & Connected to T60703 GN and polyethylene line to T6002 GN. & 9.2.8.h & LOW & 1,2 \\
\hline T6002 GN & CUB 6 & Standard Ta GN located in Cubicle 6 left DC well. & 9.2.8.i & LOW & 1,2 \\
\hline
\end{tabular}




\begin{tabular}{|c|c|c|c|c|c|}
\hline ITEM & LOCATION & DESCRIPTION & Reference & IMPACT & COMMENTS \\
\hline T-60 & TK PT 6 & Previously discussed in References 6.2.1 and 6.2.2 above. & 9.2.8.j & $\begin{array}{l}\text { See Ref. } \\
6.2 .1 \\
6.2 .2\end{array}$ & $\begin{array}{l}\text { See Ref. 6.2.1, } \\
6.2 .2\end{array}$ \\
\hline KP-607 & CUB 6 & $\begin{array}{l}\text { Located on RR-7 } 3 \text { in. diameter } \times 6 \text { in. long flanged glass pipe ( } \sim 1 \mathrm{~L} \text { volume) with } \\
\text { specially fabricated } \mathrm{Zr} \text { top and bottom plates, KOP for T-734 Transfer System. }\end{array}$ & 9.2.8.k & LOW & 1,2 \\
\hline Cubicle 6 vacuum & CUB 6 & Cubicle 6 Air Jet (C6AJ) for vacuum supply. & 9.2.8.1 & LOW & 1,2 \\
\hline Miscellaneous supplies & $\begin{array}{l}\text { CR, MUA, } \\
\text { CUB } 6\end{array}$ & Previously discussed in Reference 1.4.1.q above. & 9.2.8.m & LOW & 1,2 \\
\hline T6007 GN & CUB 6 & Standard Ta GN located in Cubicle 6 left DC well. & 9.2.9.a & LOW & 1,2 \\
\hline T73402 GN & CUB 7 & $\begin{array}{l}\text { Standard Ta GN on RR-7, inlet for T-734 with polyethylene tubing connected to T6007 } \\
\text { GN. Previously discussed in Reference 1.6.1.d above. }\end{array}$ & 9.2.9.b & LOW & 1,2 \\
\hline PS-733 & CUB 7 & Previously discussed in Reference 2.3.2.c above. & 9.2.9.c & LOW & 1,2 \\
\hline HV-T734-2 & CUB 7 & Previously discussed in Reference 1.6.1.f above. & 9.2.9.d & LOW & 1,2 \\
\hline Tank T-734 & CUB 7 & Previously discussed in Reference 1.6.1.g above. & 9.2.9.e & MED & 3,4 \\
\hline HV-T734-3 & CUB 7 & Previously discussed in Reference 1.6.1.h above. & 9.2.9.f & LOW & 1,2 \\
\hline HV-T734-1 & CUB 7 & Previously discussed in Reference 1.6.1.k above. & 9.2.9.g & LOW & 1,2 \\
\hline T73404 GN & CUB 7 & $\begin{array}{l}\text { Standard Ta GN on RR-7, outlet for T-734 with polyethylene tubing connected to C7405 } \\
\text { GN. Previously discussed in Reference 1.6.1.i above. }\end{array}$ & 9.2.9.h & LOW & 1,2 \\
\hline C7405 GN & CUB 7 & Standard Ta GN located in Cubicle 7 left DC well. & 9.2.9.i & LOW & 1,2 \\
\hline KP-735 & CUB 7 & Previously discussed in Reference 1.6.1.l above. & 9.2.9.j & LOW & 1,2 \\
\hline CU7AJ & CUB 7 & Previously discussed in Reference 1.4.1.p above. & 9.2.9.k & LOW & 1,2 \\
\hline Miscellaneous supplies & $\begin{array}{l}\text { CR, MUA, } \\
\text { CUB } 6\end{array}$ & Previously discussed in Reference 1.4.1.q above. & 9.2.9.1 & LOW & 1,2 \\
\hline 7PDV-604-1 & CUB 6 & Previously discussed in Reference 2.3.10.a above. & 9.2.10.a & LOW & 1,2 \\
\hline PS-604 & CUB 6 & Previously discussed in Reference 2.3.10.b above. & 9.2.10.b & LOW & 1,2 \\
\hline HCV-P604-1 & CUB 6 & $\begin{array}{l}\text { 2-line polyethylene block with polyethylene tubing and a PVC ball valve connected from } \\
\text { the left side to P65301 GN. Previously discussed in Reference 2.3.10.c above. }\end{array}$ & 9.2.10.c & LOW & 1,2 \\
\hline P65301 GN & CUB 6 & $\begin{array}{l}\text { Standard Zr GN on BR-6, inlet for P-653 with polyethylene tubing connected from 2-line } \\
\text { block at HCV-P604-1 position on BR-6. Previously discussed in Reference } \mathbf{2 . 3 . 1 0 . d} \text { above. }\end{array}$ & 9.2.10.d & LOW & 1,2 \\
\hline P-653 pump & CUB 6 & Previously discussed in Reference 2.3.10.e above. & 9.2.10.e & MED & $5,6,8$ \\
\hline P65302 GN & CUB 6 & $\begin{array}{l}\text { Standard Zr GN on BR-6, outlet for P-653 with PVC ball valve and polyethylene tubing } \\
\text { connected to special FDV-T604 on BR-6. Previously discussed in Reference 2.3.10.f } \\
\text { above. }\end{array}$ & 9.2.10.f & LOW & 1,2 \\
\hline FDV-T604 & CUB 6 & $\begin{array}{l}\text { FDV special polyethylene block located on BR-6 with polyethylene line connected to } \\
\text { C4C601 GN. Previously discussed in Reference } \mathbf{2 . 3 . 1 0 . g} \text { above. }\end{array}$ & 9.2.10.g & LOW & 1,2 \\
\hline \begin{tabular}{|l|l|} 
C4C601 GN \\
\end{tabular} & CUB 6 & Previously discussed in Reference 2.3.2.h above. & 9.2.10.h & LOW & 1,2 \\
\hline C4C601 jumper & $\begin{array}{l}\text { TK PIT 4, 5, } \\
\text { and } 6\end{array}$ & Previously discussed in Reference 2.3.2.i above. & $9.2 .10 . \mathrm{i}$ & LOW & 1,2 \\
\hline
\end{tabular}




\begin{tabular}{|c|c|c|c|c|c|}
\hline ITEM & LOCATION & DESCRIPTION & Reference & IMPACT & COMMENTS \\
\hline C4C601 jumper & CUB 4 & Previously discussed in Reference 2.3.2.j above. & $9.2 .10 . \mathrm{j}$ & LOW & 1,2 \\
\hline C4C601 GN & CUB 4 & Previously discussed in Reference 2.3.2.k above. & $9.2 .10 . \mathrm{k}$ & LOW & 1,2 \\
\hline T4003 GN & CUB 4 & Previously discussed in Reference 3.6.2.I above. & 9.2.10.1 & LOW & 1,2 \\
\hline T4013 GN & CUB 4 & Previously discussed in Reference 3.6.2.m above. & 9.2.10.m & LOW & 1,2 \\
\hline 7PDV-604-2 & CUB 6 & Previously discussed in Reference 2.3.10.h above. & 9.2.10.n & LOW & 1,2 \\
\hline HCV-P604-2 & CUB 6 & Previously discussed in Reference 2.3.10.i above. & & LOW & 1,2 \\
\hline Miscellaneous supplies & $\begin{array}{l}\text { CR, MUA, } \\
\text { CUB } 6\end{array}$ & Previously discussed in Reference 1.4.1.q above. & 9.2.10.i & LOW & 1,2 \\
\hline Tank T-40 & TK PIT 4 & $\begin{array}{l}\text { Nominal } 63 \text { L HC shell with Ta liner evaporator tank. T-40 has an annulus filled with Hg } \\
\text { for increases heat transfer capability. }\end{array}$ & 9.2.11.a & HIGH & 11,12 \\
\hline $\begin{array}{l}\text { T-40 LL, DR, and PR } \\
\text { instr. }\end{array}$ & CR, MUA & $\begin{array}{l}\text { Foxboro pneumatic instrument is located in the CR and the DP cell and associated } \\
\text { polyethylene tubing is located in the MUA. The LL, DR, and PR lines are constructed of Ta } \\
\text { and originate in T-40 in Tank Pit 4, travel through the LAA, and are tied to the } \\
\text { polyethylene tubing that goes to the DP transmitters in the MUA. }\end{array}$ & 9.2.11.b & HIGH & $9,10,11,12$ \\
\hline T-40 sparger & $\begin{array}{l}\text { MUA, CR, TK } \\
\text { PIT } 4 \\
\end{array}$ & $\begin{array}{l}\text { There's a push button switch in CR to activate a solenoid in the MUA. An in-line orifice } \\
\text { controls air flow into the tank. }\end{array}$ & 9.2.11.c & LOW & $1,2,4$ \\
\hline $\begin{array}{l}\text { T-40 process and } \\
\text { instrument lines }\end{array}$ & TK PIT 4 & Lines are constructed of Ta and provide solution transfer routes into and out of T- 40 . & 9.2.11.d & HIGH & $9,10,11,12$ \\
\hline $\begin{array}{l}\text { T-40 vacuum } \\
\text { accumulator }\end{array}$ & CUB 4 & $\begin{array}{l}3 \text { in. diameter } \times 6 \text { in. long flanged glass pipe ( } \sim 1 \mathrm{~L} \text { volume) with specially fabricated } \mathrm{Zr} \text { top } \\
\text { and bottom plates. }\end{array}$ & 9.2.11.e & NCBU & N/A \\
\hline SA-T40 needle block & CUB 4 & Specially fabricated Zr block with 2 needles for pulling samples. & 9.2.11.e & NCBU & N/A \\
\hline $\begin{array}{l}\text { Auxiliary glass sample } \\
\text { flask }\end{array}$ & CUB 4 & $\begin{array}{l}1 \mathrm{~L} \text { glass bottle with 2-line stopper outfitted with polyethylene tubing connected to SA-T40 } \\
\text { position on CUB } 4 \text { sample rack for sampling T- } 40 \text { and for connection to vacuum source. }\end{array}$ & 9.2.11.e & LOW & 1,2 \\
\hline $\begin{array}{l}\text { SA-T40 special 1-line } \\
\text { block }\end{array}$ & CUB 4 & $\begin{array}{l}\text { Special 1-line polyethylene block with } 3 / 8 \text { in. metal tubing on one side installed at SA-T40 } \\
\text { position on CUB } 4 \text { sample rack for sampling T-40 using auxiliary glass sample flask. }\end{array}$ & 9.2.11.e & LOW & 1,2 \\
\hline T-40 thermocouples & $\begin{array}{l}\text { CR, MUA, TK } \\
\text { PIT } 4\end{array}$ & Thermocouples, leads, and jumpers to Control Room Instruments. & 9.2.12.a & HIGH & $9,10,11,12$ \\
\hline $\begin{array}{l}\text { T-40 steam lines and } \\
\text { valves }\end{array}$ & $\begin{array}{l}\text { MUA, CR, TK } \\
\text { PIT } 4 \\
\end{array}$ & $\begin{array}{l}\text { Steam lines to the tanks, manual valves in MUA, and electrically operated solenoid valve in } \\
\text { LAA. }\end{array}$ & 9.2.12.b & HIGH & $9,10,11,12$ \\
\hline PIC-T40 & CR & Pressure controller for PCV-40 that controls steam supply to T-40 heating/cooling jacket. & 9.2.12.c & LOW & 1,2 \\
\hline PCV-T-40 & MUA & Pressure control valve that controls steam supply to T-40 heating/cooling jacket. & 9.2.12.c & LOW & 1,2 \\
\hline $\begin{array}{l}\text { T-40 RCW lines and } \\
\text { valves }\end{array}$ & CR, TK PIT 4 & Manual valves in MUA and electrically operated solenoid valve in LAA. & 9.2.12.d & HIGH & $9,10,11,12$ \\
\hline $\mathrm{H}-40$ & CR,TK PIT 4 & Manual valves in MUA and electrically operated solenoid valve in LAA. & 9.2.12.e & HIGH & $9,10,11,12$ \\
\hline
\end{tabular}




\begin{tabular}{|c|c|c|c|c|c|}
\hline ITEM & LOCATION & DESCRIPTION & Reference & IMPACT & COMMENTS \\
\hline T-40 VOG & TK PIT 4 & Condensate moves through VOG line and collects in a condensate collection tank. & 9.2.12.f & LOW & 3 \\
\hline M-704 & MUA & Previously discussed in Reference 1.4.2 above. & 9.2.13.a & LOW & 1,2 \\
\hline T-60 sampling (strip) & CUB 6 & Previously discussed in Reference 6.2.1.e above. & 9.2.13.b & LOW & 1,2 \\
\hline T-60 to T-604 transfer & $\begin{array}{l}\text { CUB 6, TK } \\
\text { PIT } 6\end{array}$ & Previously discussed in Reference 9.2.9 above. & 9.2.13.c & \begin{tabular}{|l} 
See Ref. \\
9.2 .9 \\
\end{tabular} & See Ref. 9.2.9 \\
\hline T-60 sampling (org.) & CUB 6 & Previously discussed in Reference 6.2.1.e above. & 9.2.13.d & LOW & 1,2 \\
\hline Tank T-40 & TK PIT 4 & Previously discussed in References 9.2.11 and 9.2.12 above. & 9.2.13.e & \begin{tabular}{|l} 
See Ref. \\
9.2.11, \\
9.2.12
\end{tabular} & \begin{tabular}{|l|} 
See Ref. \\
9.2.11, 9.2.12
\end{tabular} \\
\hline M-704 & MUA & Previously discussed in Reference 1.4.2 above. & 9.2.14.a & LOW & 1,2 \\
\hline T-604 sampling (strip) & CUB 6 & Previously discussed in Reference 2.3.8.e above. & 9.2.14.b & LOW & 1,2 \\
\hline T-604 to T-40 transfer & $\begin{array}{l}\text { CUB 6, TK } \\
\text { PIT 6, TK PIT } \\
4 \\
\end{array}$ & Previously discussed in Reference 9.2.10 above. & 9.2.14.c & \begin{tabular}{|l} 
See Ref. \\
9.2 .10
\end{tabular} & See Ref. 9.2.10 \\
\hline T-604 sampling (org.) & CUB 6 & Previously discussed in Reference 2.3.8.e above. & 9.2.14.d & LOW & 1,2 \\
\hline Tank T-40 & TK PIT 4 & Previously discussed in References 9.2.11 and 9.2.12 above. & 9.2.14.e & \begin{tabular}{|l} 
See Ref. \\
9.2.11, \\
9.2.12 \\
\end{tabular} & \begin{tabular}{|l} 
See Ref. \\
$9.2 .11,9.2 .12$
\end{tabular} \\
\hline Tank T-77 & TK PIT 7 & Previously discussed in Reference 9.2.5 above. & 9.2.15.a, & \begin{tabular}{|l|} 
See Ref. \\
9.2 .5 \\
\end{tabular} & See Ref. 9.2.5 \\
\hline Tank T-60 & TK PIT6 & Previously discussed in References 6.2.1 and 6.2.2 above. & 9.2.15.a & $\begin{array}{l}\text { See Ref. } \\
6.2 .1 \\
6.2 .2 \\
\end{array}$ & $\begin{array}{l}\text { See Ref. 6.2.1, } \\
6.2 .2\end{array}$ \\
\hline Tank T-604 & TK PIT6 & Previously discussed in References 2.3.8 and 2.3.9 above. & 9.2.15.a & $\begin{array}{l}\text { See Ref. } \\
2.3 .8 \\
2.3 .9 \\
\end{array}$ & $\begin{array}{l}\text { See Ref. 2.3.8, } \\
\text { 2.3.9 }\end{array}$ \\
\hline T-77 sampling & CUB 7 & Previously discussed in Reference 9.2.5.e above. & 9.2.15.b & LOW & 1,2 \\
\hline T-60 sampling & CUB 6 & Previously discussed in Reference 6.2.1.e above. & 9.2.15.b & LOW & 1,2 \\
\hline T-604 sampling & CUB 6 & Previously discussed in Reference 2.3.8.e above. & 9.2.15.b & LOW & 1,2 \\
\hline $\begin{array}{l}\text { T-77, T-60, and T-604 } \\
\text { waste organic transfer } \\
\text { routes }\end{array}$ & $\begin{array}{l}\text { CUB } 6 \text { and 7, } \\
\text { TK PIT } 6 \text { and } \\
\text { 7, WASTE TK } \\
\text { PIT } \\
\end{array}$ & Discussed in References 9.2.16, 9.2.17, 1nd 9.2.18 below. & 9.2.15.c & \begin{tabular}{|l} 
See Ref. \\
9.2 .16 \\
9.2 .17 \\
9.2 .18 \\
\end{tabular} & $\begin{array}{l}\text { See Ref. } \\
9.2 .16,9.2 .17 \text {, } \\
9.2 .18\end{array}$ \\
\hline $\begin{array}{l}\text { Tank M-703 transfer } \\
\text { system }\end{array}$ & MUA & Previously discussed in Reference 2.1.3 above. & 9.2.15.d & \begin{tabular}{|l} 
See Ref. \\
2.1 .3 \\
\end{tabular} & See Ref. 2.1.3 \\
\hline
\end{tabular}




\begin{tabular}{|c|c|c|c|c|c|}
\hline ITEM & LOCATION & DESCRIPTION & Reference & IMPACT & COMMENTS \\
\hline T-77 waste jet & $\begin{array}{l}\text { MUA, TK PIT } \\
7\end{array}$ & T-77 waste jet (J-T77A) to F-115 (or alternate transfer route outlined in Reference 9.2.16). & 9.2.15.e & MED & 4,12 \\
\hline T-60 waste jet & $\begin{array}{l}\text { MUA, TK PIT } \\
6\end{array}$ & T-60 waste jet (J-T60) to F-115 (or alternate transfer route outlined in Reference 9.2.17). & 9.2.15.e & MED & 4,12 \\
\hline T-604 waste jet & $\begin{array}{l}\text { MUA, TK PIT } \\
6 \\
\end{array}$ & T-604 waste jet (J-T604) to F-115 (or alternate transfer route outlined in Reference 9.2.18). & 9.2.15.e & MED & 4,12 \\
\hline T-60 evaporation & $\begin{array}{l}\text { CR, MUA, TK } \\
\text { PIT } 6\end{array}$ & Previously discussed in References 6.2.1 and 6.2.2 above. & 9.2.15.f & $\begin{array}{l}\text { See Ref. } \\
6.2 .1 \\
6.2 .2 \\
\end{array}$ & $\begin{array}{l}\text { See Ref. 6.2.1, } \\
6.2 .2\end{array}$ \\
\hline T-604 evaporation & $\begin{array}{l}\text { CR, MUA, TK } \\
\text { PIT } 6\end{array}$ & Previously discussed in References 2.3.8 and 2.3.9 above. & 9.2.15.f & $\begin{array}{l}\text { See Ref. } \\
2.3 .8 \text {, } \\
2.3 .9\end{array}$ & $\begin{array}{l}\text { See Ref. 2.3.8, } \\
2.3 .9\end{array}$ \\
\hline $\begin{array}{l}\text { T-77, T-60, and T-604 } \\
\text { waste jets }\end{array}$ & \begin{tabular}{|l|} 
MUA, TK \\
PIT6, TK PIT 7 \\
\end{tabular} & Previously discussed in Reference 9.2.15.e above. & 9.2.15.g & MED & 4.12 \\
\hline T7707 GN & \begin{tabular}{|l|l} 
CUB 7 & \\
\end{tabular} & Previously discussed in Reference 9.2.9.a above. & 9.2.16.a & LOW & 1,2 \\
\hline T73402 GN & CUB 7 & Previously discussed in Reference 1.6.1.d above. & 9.2.16.b & LOW & 1,2 \\
\hline PS-733 & CUB 7 & Previously discussed in Reference 1.6.1.e above. & 9.2.16.c & LOW & 1,2 \\
\hline HV-T734-2 & CUB 7 & Previously discussed in Reference 1.6.1.f above. & 9.2.16.d & LOW & 1,2 \\
\hline Tank T-734 & CUB 7 & Previously discussed in Reference 1.6.1.g above. & 9.2.16.e & MED & 3,4 \\
\hline HV-T734-3 & CUB 7 & Previously discussed in Reference 1.6.1.h above. & 9.2.16.f & LOW & 1,2 \\
\hline HV-T734-1 & CUB 7 & Previously discussed in Reference 1.6.1.k above. & 9.2.16.g & LOW & 1,2 \\
\hline T73404 GN & CUB 7 & $\begin{array}{l}\text { Previously discussed in Reference 1.6.1.i above with polyethylene tubing connected to } \\
\text { C7405 GN. }\end{array}$ & 9.2.16.h & LOW & 1,2 \\
\hline $\begin{array}{l}\text { SA-F126 special 1-line } \\
\text { block }\end{array}$ & CUB 7 & $\begin{array}{l}\text { Special 1-line polyethylene block with 3/8 in. metal tubing on one side installed at SA-F126 } \\
\text { position on CUB } 7 \text { sample rack used for transferring material in to F-126. }\end{array}$ & 9.2.16.i & LOW & 1,2 \\
\hline KP-735 & CUB 7 & Previously discussed in Reference 1.6.1.I above. & 9.2.16.j & LOW & 1,2 \\
\hline CU7AJ & CUB 7 & Previously discussed in Reference 1.4.1.p above. & 9.2.16.k & LOW & 1,2 \\
\hline Miscellaneous supplies & $\begin{array}{l}\text { CR, MUA, } \\
\text { CUB } 7\end{array}$ & Previously discussed in Reference 1.4.1.q above. & 9.2.16.1 & LOW & 1,2 \\
\hline T6007 GN & CUB 6 & Previously discussed in Reference 9.2.9.a above. & 9.2.17.a & LOW & 1,2 \\
\hline T73402 GN & CUB 7 & Previously discussed in Reference 1.6.1.d above. & 9.2.17.b & LOW & 1,2 \\
\hline PS-733 & CUB 7 & Previously discussed in Reference 1.6.1.e above. & 9.2.17.c & LOW & 1,2 \\
\hline HV-T734-2 & CUB 7 & Previously discussed in Reference 1.6.1.f above. & 9.2.17.d & LOW & 1,2 \\
\hline Tank T-734 & CUB 7 & Previously discussed in Reference 1.6.1.g above. & 9.2.17.e & MED & 3,4 \\
\hline HV-T734-3 & CUB 7 & Previously discussed in Reference 1.6.1.h above. & 9.2.17.f & LOW & 1,2 \\
\hline HV-T734-1 & CUB 7 & Previously discussed in Reference 1.6.1.k above. & 9.2.17.g & LOW & 1,2 \\
\hline T73404 GN & CUB 7 & Previously discussed in Reference 1.6.1.i above. & 9.2.17.h & LOW & 1,2 \\
\hline
\end{tabular}




\begin{tabular}{|c|c|c|c|c|c|}
\hline ITEM & LOCATION & DESCRIPTION & Reference & IMPACT & COMMENTS \\
\hline $\begin{array}{l}\text { SA-F126 special 1-line } \\
\text { block }\end{array}$ & CUB 7 & Previously discussed in Reference 9.2.16.i above. & 9.2.17.i & LOW & 1,2 \\
\hline KP-735 & CUB 7 & Previously discussed in Reference 1.6.1.I above. & 9.2.17.j & LOW & 1,2 \\
\hline CU7AJ & CUB 7 & Previously discussed in Reference 1.4.1.p above. & 9.2.17.k & LOW & 1,2 \\
\hline Miscellaneous supplies & $\begin{array}{l}\text { CR, MUA, } \\
\text { CUB 6,7 } \\
\end{array}$ & Previously discussed in Reference 1.4.1.q above. & 9.2.17.1 & LOW & 1,2 \\
\hline 7PDV-604-1 & CUB 6 & Previously discussed in Reference 9.2.10.a above. & 9.2.18.a & LOW & 1,2 \\
\hline PS-604 & CUB 6 & Previously discussed in Reference 2.3.17.b above. & 9.2.18.b & LOW & 1,2 \\
\hline T73402 GN & CUB 7 & Previously discussed in Reference 1.6.1.d above. & 9.2.18.c & LOW & 1,2 \\
\hline PS-733 & CUB 7 & Previously discussed in Reference 1.6.1.e above. & 9.2.18.d & LOW & 1,2 \\
\hline HV-T734-2 & CUB 7 & Previously discussed in Reference 1.6.1.f above. & 9.2.18.e & LOW & 1,2 \\
\hline Tank T-734 & CUB 7 & Previously discussed in Reference 1.6.1.g above. & 9.2.18.f & MED & 3.4 \\
\hline HV-T734-3 & CUB 7 & Previously discussed in Reference 1.6.1.h above. & 9.2.18.g & LOW & 1,2 \\
\hline HV-T734-1 & CUB 7 & Previously discussed in Reference 1.6.1.k above. & 9.2.18.h & LOW & 1,2 \\
\hline T73404 GN & CUB 7 & Previously discussed in Reference 1.6.1.i above. & 9.2.18.i & LOW & 1,2 \\
\hline $\begin{array}{l}\text { SA-F126 special 1-line } \\
\text { block }\end{array}$ & CUB 7 & Previously discussed in Reference 9.2.16.i above. & 9.2.18.j & LOW & 1,2 \\
\hline KP-735 & CUB 7 & Previously discussed in Reference 1.6.1.I above. & 9.2.18.k & LOW & 1,2 \\
\hline CU7AJ & CUB 7 & Previously discussed in Reference 1.4.1.p above. & 9.2.17.k & LOW & 1,2 \\
\hline Miscellaneous supplies & $\begin{array}{l}\text { CR, MUA, } \\
\text { CUB 6,7 }\end{array}$ & Previously discussed in Reference 1.4.1.q above. & 9.2.18.m & LOW & 1,2 \\
\hline T-40 sampling & CUB 4 & Previously discussed in Reference 9.2.11.e above. & 9.2.19.a & LOW & 1,2 \\
\hline T-40 evaporator & $\begin{array}{l}\text { CR, MUA, TK } \\
\text { PIT } 4\end{array}$ & Previously discussed in Reference 9.2.12 above. & 9.2.19.b & $\begin{array}{l}\text { See Ref. } \\
9.2 .11 \\
9.2 .12\end{array}$ & $\begin{array}{l}\text { See Ref. } \\
9.2 .11,9.2 .12\end{array}$ \\
\hline Tank F-126 & $\begin{array}{l}\text { WASTE TK } \\
\text { PT }\end{array}$ & Nominal 3531 L tank constructed of stainless steel with stainless steel probes. & 9.3.1.a & HIGH & 11,12 \\
\hline F-126 LL instr. & CR, MUA & $\begin{array}{l}\text { Foxboro pneumatic instrument is located in the CR and the DP cell and associated } \\
\text { polyethylene tubing is located in the MUA. The LL lines are constructed of stainless steel } \\
\text { and originate in F-126 in the waste tank pit, travel through the LAA, and are tied to the } \\
\text { polyethylene tubing that goes to the DP transmitter in the MUA. }\end{array}$ & 9.3.1.b & HIGH & $9,10,11,12$ \\
\hline F-126 sparger & $\begin{array}{l}\text { MUA, CR, } \\
\text { WASTE TK } \\
\text { PT }\end{array}$ & $\begin{array}{l}\text { There's an electric toggle switch in CR to activate a solenoid in the MUA which in turns } \\
\text { opens HCV-F126-6. An in-line orifice controls air flow into the tank. }\end{array}$ & 9.3.1.c & LOW & $1,2,4$ \\
\hline
\end{tabular}




\begin{tabular}{|c|c|c|c|c|c|}
\hline ITEM & LOCATION & DESCRIPTION & Reference & IMPACT & COMMENTS \\
\hline $\begin{array}{l}\text { F-126 process and } \\
\text { instrument lines }\end{array}$ & $\begin{array}{l}\text { WASTE TK } \\
\text { PT }\end{array}$ & $\begin{array}{l}\text { Lines are constructed of stainless steel and provide solution transfer routes into and out of } \\
\text { F-126. }\end{array}$ & 9.3.1.d & HIGH & $9,10,11,12$ \\
\hline $\begin{array}{l}\text { F-126 vacuum } \\
\text { accumulator }\end{array}$ & CUB 7 & $\begin{array}{l}3 \text { in. diameter } \times 6 \text { in. long flanged glass pipe ( } \sim 1 \mathrm{~L} \text { volume) with specially fabricated } \mathrm{Zr} \text { top } \\
\text { and bottom plates. }\end{array}$ & 9.3.1.e & NCBU & N/A \\
\hline SA-F126 needle block & CUB 7 & Specially fabricated Zr block with 2 needles for pulling samples . & 9.3.1.e & NCBU & N/A \\
\hline $\begin{array}{l}\text { Auxiliary glass sample } \\
\text { flask }\end{array}$ & CUB 7 & $\begin{array}{l}1 \text { L glass bottle with 2-line stopper outfitted with polyethylene tubing connected to SA- } \\
\text { F126 position on CUB } 7 \text { sample rack for sampling F-126 and for connection to vacuum } \\
\text { source. }\end{array}$ & 9.3.1.e & LOW & 1,2 \\
\hline $\begin{array}{l}\text { SA-F126 special 1-line } \\
\text { block }\end{array}$ & CUB 7 & $\begin{array}{l}\text { Special 1-line polyethylene block with 3/8 in. metal tubing on one side installed at SA-F126 } \\
\text { position on CUB } 7 \text { sample rack for sampling F-126 using auxiliary glass sample flask. }\end{array}$ & 9.3.1.e & LOW & 1,2 \\
\hline F-126 sampling & CUB 7 & Previously discussed in Reference 9.3.1.e above. & 9.3.2.a & LOW & 1,2 \\
\hline $\begin{array}{l}\text { Thorium addition to } \\
\text { F-126 }\end{array}$ & CUB 7 & Added down F126A06 line in Cubicle 7 left DC well. & 9.3.2.g & LOW & 1,2 \\
\hline $\begin{array}{l}\mathrm{NaOH} \text { addition to } \\
\text { F-126 } \\
\end{array}$ & $\begin{array}{l}\text { TK PIT 7, } \\
\text { MUA }\end{array}$ & $\begin{array}{l}\text { Addition made from Tank M-40 in the MUA to F-126 solution addition line. M- } 40 \text { is } \\
\text { discussed in Reference } 9.6 \text { below. }\end{array}$ & 9.3.2.h & \begin{tabular}{|l|} 
See Ref \\
9.6 \\
\end{tabular} & See Ref 9.6 \\
\hline $\mathrm{J}-126 \mathrm{~B}$ & $\begin{array}{l}\text { CR, MUA, } \\
\text { LAA }\end{array}$ & Steam jet used for transfer of F-126 contents to F-1800 waste tank. & 9.3.2.j & HIGH & $9,10,12$ \\
\hline $\begin{array}{l}\text { Melton Valley Waste } \\
\text { System monitoring } \\
\text { equipment }\end{array}$ & $\begin{array}{l}\text { TA, CR, MUA, } \\
\text { LAA }\end{array}$ & $\begin{array}{l}\text { Electronic monitoring equipment with sensors located in multiple strategic locations } \\
\text { through the waste disposal system. }\end{array}$ & 9.3.2.k & MED & 5,6 \\
\hline $\begin{array}{l}\text { F-126 transfer system } \\
\text { flush }\end{array}$ & LAA & Process water system tie in to F-126 waste transfer line. & 9.3.2.1 & LOW & 1,2 \\
\hline Tank F-111 & $\begin{array}{l}\text { WASTE TK } \\
\text { PT }\end{array}$ & Nominal 1800 L tank constructed of stainless steel with stainless steel probes. & 9.4.1.a & HIGH & 11,12 \\
\hline F-111 LL instr. & CR, MUA & $\begin{array}{l}\text { Foxboro pneumatic instrument is located in the CR and the DP cell and associated } \\
\text { polyethylene tubing is located in the MUA. The LL lines are constructed of stainless steel } \\
\text { and originate in F-111 in the waste tank pit, travel through the LAA, and are tied to the } \\
\text { polyethylene tubing that goes to the DP transmitter in the MUA. }\end{array}$ & 9.4.1.b & HIGH & $9,10,11,12$ \\
\hline \begin{tabular}{|l} 
F-111 sparger \\
\end{tabular} & $\begin{array}{l}\text { MUA, CR, } \\
\text { WASTE TK } \\
\text { PT }\end{array}$ & $\begin{array}{l}\text { There's an electric toggle switch in CR to activate a solenoid in the MUA which in turns } \\
\text { opens HCV-F111-6. An in-line orifice controls air flow into the tank. }\end{array}$ & 9.4.1.c & LOW & $1,2,4$ \\
\hline $\begin{array}{l}\text { F-111 process and } \\
\text { instrument lines }\end{array}$ & $\begin{array}{l}\text { WASTE TK } \\
\text { PT }\end{array}$ & $\begin{array}{l}\text { Lines are constructed of stainless steel and provide solution transfer routes into and out of } \\
\text { F-111. }\end{array}$ & 9.4.1.d & HIGH & $9,10,11,12$ \\
\hline \begin{tabular}{|l|} 
F-111 vacuum \\
accumulator
\end{tabular} & CUB 7 & $\begin{array}{l}3 \text { in. diameter } \times 6 \text { in. long flanged glass pipe ( } \sim 1 \mathrm{~L} \text { volume) with specially fabricated } \mathrm{Zr} \text { top } \\
\text { and bottom plates. }\end{array}$ & 9.4.1.e & NCBU & N/A \\
\hline
\end{tabular}




\begin{tabular}{|c|c|c|c|c|c|}
\hline ITEM & LOCATION & DESCRIPTION & Reference & IMPACT & COMMENTS \\
\hline SA-F111 needle block & CUB 7 & Specially fabricated Zr block with two needles for pulling samples. & 9.4.1.e & NCBU & N/A \\
\hline $\begin{array}{l}\text { Auxiliary glass sample } \\
\text { flask }\end{array}$ & CUB 7 & $\begin{array}{l}1 \text { L glass bottle with 2-line stopper outfitted with polyethylene tubing connected to SA- } \\
\text { F111 position on CUB } 7 \text { sample rack for sampling F-111 and for connection to vacuum } \\
\text { source. }\end{array}$ & 9.4.1.e & LOW & 1,2 \\
\hline $\begin{array}{l}\text { SA-F111 special 1-line } \\
\text { block }\end{array}$ & CUB 7 & $\begin{array}{l}\text { Special 1-line polyethylene block with 3/8 in. metal tubing on one side installed at SA-F111 } \\
\text { position on CUB } 7 \text { sample rack for sampling F-111 using auxiliary glass sample flask. }\end{array}$ & 9.4.1.e & LOW & 1,2 \\
\hline Tank T-34 & TK PIT 3 & Nominal 154.3 L tank constructed of HC with HC probes. & 9.5.1.a & HIGH & 11,12 \\
\hline $\begin{array}{l}\text { T-34 LL, DR, and PR } \\
\text { instr. }\end{array}$ & CR, MUA & $\begin{array}{l}\text { Foxboro pneumatic instrument is located in the CR and the DP cell and associated } \\
\text { polyethylene tubing is located in the MUA. The LL, DR, and PR lines are constructed of Ta } \\
\text { and originate in T- } 34 \text { in Tank Pit 3, travel through the LAA, and are tied to the } \\
\text { polyethylene tubing that goes to the DP transmitters in the MUA. }\end{array}$ & 9.5.1.b & HIGH & $9,10,11,12$ \\
\hline T-34 sparger & $\begin{array}{l}\text { MUA, CR, TK } \\
\text { PIT } 3\end{array}$ & $\begin{array}{l}\text { There's a push button switch in CR to activate a solenoid in the MUA. An in-line orifice } \\
\text { controls air flow into the tank. }\end{array}$ & 9.5.1.c & LOW & $1,2,4$ \\
\hline $\begin{array}{l}\text { T-34 process and } \\
\text { instrument lines }\end{array}$ & TK PIT 3 & Lines are constructed of HC and provide solution transfer routes into and out of T-34. & 9.5.1.d & HIGH & $9,10,11,12$ \\
\hline $\begin{array}{l}\text { T-34 vacuum } \\
\text { accumulator }\end{array}$ & CUB 6 & $\begin{array}{l}3 \text { in. diameter } \times 6 \text { in. long flanged glass pipe ( } \sim 1 \mathrm{~L} \text { volume) with specially fabricated } \mathrm{Zr} \text { top } \\
\text { and bottom plates. }\end{array}$ & 9.5.1.e & NCBU & N/A \\
\hline SA-T34 needle block & CUB 6 & Specially fabricated Zr block with two needles for pulling samples. & 9.5.1.e & NCBU & N/A \\
\hline $\begin{array}{l}\text { Auxiliary glass sample } \\
\text { flask }\end{array}$ & CUB 6 & $\begin{array}{l}1 \mathrm{~L} \text { glass bottle with 2-line stopper outfitted with polyethylene tubing connected to SA-T34 } \\
\text { position on CUB } 6 \text { sample rack for sampling T-34 and for connection to vacuum source. }\end{array}$ & 9.5.1.e & LOW & 1,2 \\
\hline $\begin{array}{l}\text { SA-T34 special 1-line } \\
\text { block }\end{array}$ & CUB 6 & $\begin{array}{l}\text { Special 1-line polyethylene block with 3/8 in. metal tubing on one side installed at SA-T34 } \\
\text { position on CUB } 6 \text { sample rack for sampling T-34 using auxiliary glass sample flask. }\end{array}$ & 9.5.1.e & LOW & 1,2 \\
\hline Tank M-40 & MUA & $\begin{array}{l}\text { Nominal } 50 \text { L PVC tank used for cold chemical additions to T-34, F-111, F-115, and F- } \\
126 .\end{array}$ & 9.6 .1 & LOW & 1,2 \\
\hline V-M40-1 & MUA & Ball valve, vent line to VOG system. & 9.6.1.a & LOW & 1,2 \\
\hline V-M40-2 & MUA & Ball valve, solution addition line through the funnel. & 9.6.1.b & LOW & 1,2 \\
\hline V-M40-3 & MUA & Ball valve, 5-10 psig air pressure supply line. & 9.6.1.c & LOW & 1,2 \\
\hline V-M40-4 & MUA & Ball valve, outlet line/drain. & 9.6.1.d & LOW & 1,2 \\
\hline V-M40-6 & MUA & Ball valve, inlet line from M-500. & 9.6.1.e & LOW & 1,2 \\
\hline V-M40-7 & MUA & Ball valve, outlet line to header for T-34 and waste tank header. & 9.6.1.f & LOW & 1,2 \\
\hline V-M40-8 & MUA & Ball valve, outlet line from header to T-34. & 9.6.1.g & LOW & 1,2 \\
\hline V-M40-10 & MUA & Ball valve, sight glass valve at bottom of sight glass. & 9.6.1.h & LOW & 1,2 \\
\hline V-M40-13 & MUA & Ball valve, outlet line. & 9.6.1.i & LOW & 1,2 \\
\hline V-M40-14 & MUA & Ball valve, outlet line from header to waste tank header. & 9.6.1.j & LOW & 1,2 \\
\hline
\end{tabular}


10.0 Miscellaneous Process Operations

\begin{tabular}{|c|c|c|c|c|c|}
\hline ITEM & LOCATION & DESCRIPTION & Reference & IMPACT & COMMENTS \\
\hline $\begin{array}{l}\text { In-cell demineralized } \\
\text { water }\end{array}$ & $\begin{array}{l}\text { CUB 4, 5, 6, or } \\
7\end{array}$ & In-cell demineralized water supply for Cubicles $4,5,6$, or 7. & 10.1.1.b & LOW & 3,4 \\
\hline MUA tank & MUA & Any appropriate MUA tank compatible with the acid used for the flushing. & 10.1.1.e & LOW & 3,4 \\
\hline $\begin{array}{l}\text { Designated cubicle } \\
\text { solution addition line }\end{array}$ & $\begin{array}{l}\text { CUB 4, 5, 6, or } \\
7\end{array}$ & Cubicle solution addition lines for Cubicles 4, 5, 6, or 7 used as required. & 10.1.1.e & LOW & 3,4 \\
\hline Tank T-66 & TK PIT 6 & Miscellaneous solution collection tank. Discussed in Reference 10.2.1 below. & 10.1.1.f & \begin{tabular}{|l} 
See Ref. \\
10.2 .1 \\
\end{tabular} & See Ref. 10.2.1 \\
\hline Tank T-79 & TK PIT 7 & Miscellaneous solution collection tank. Discussed in Reference 10.2.2 below. & 10.1.1.f & \begin{tabular}{|l|} 
See Ref. \\
10.2 .2 \\
\end{tabular} & See Ref. 10.2.2 \\
\hline Tank T-21 & TK PIT 2 & Previously discussed in References 2.2.3 above. & 10.1.1.g & \begin{tabular}{|l} 
See Ref. \\
2.2 .3
\end{tabular} & See Ref. 2.2.3 \\
\hline Tank T-23 & TK PIT 2 & Previously discussed in References 2.3.4 above. & 10.1.1.g & \begin{tabular}{|l|} 
See Ref. \\
2.2 .4 \\
\end{tabular} & See Ref. 2.2.4 \\
\hline F-115 waste & \begin{tabular}{|l|l|} 
WASTE \\
TANK PIT
\end{tabular} & Previously discussed in Reference 9.2 above. & 10.1.1.g & \begin{tabular}{|l} 
See Ref. \\
9.2 \\
\end{tabular} & See Ref.9.2 \\
\hline MUA tank & MUA & Any appropriate MUA tank compatible with the acid used for the flushing. & 10.1.2.c & LOW & $1,2,3,4$ \\
\hline $\begin{array}{l}\text { Appropriate addition } \\
\text { line }\end{array}$ & $\begin{array}{l}\text { CUB 4, 5, 6, or } \\
7\end{array}$ & $\begin{array}{l}\text { Appropriate addition line to access the equipment to be flushed in Cubicles 4, 5, 6, or } 7 \\
\text { used as required. }\end{array}$ & 10.1.2.c & LOW & 3,4 \\
\hline Tank T-66 & TK PIT 6 & Miscellaneous solution collection tank. Discussed in Reference 10.2.1 below. & 10.1.2.d & \begin{tabular}{|l|} 
See Ref. \\
10.2 .1 \\
\end{tabular} & See Ref. 10.2.1 \\
\hline Tank T-79 & TK PIT 7 & Miscellaneous solution collection tank. Discussed in Reference 10.2.2 below. & 10.1.2.d & \begin{tabular}{|l|} 
See Ref. \\
10.2 .2 \\
\end{tabular} & See Ref. 10.2.2 \\
\hline Tank T-21 & TK PIT 2 & Previously discussed in References 2.2.3 above. & 10.1.2.e & \begin{tabular}{|l|} 
See Ref. \\
2.2 .3 \\
\end{tabular} & See Ref. 2.2.3 \\
\hline Tank T-23 & TK PIT 2 & Previously discussed in References 2.3.4 above. & 10.1.2.e & \begin{tabular}{|l|} 
See Ref. \\
2.2 .4
\end{tabular} & See Ref. 2.2.4 \\
\hline F-115 Waste & \begin{tabular}{|l} 
WASTE \\
TANK PIT \\
\end{tabular} & Previously discussed in Reference 9.2 above. & 10.1.2.e & \begin{tabular}{|l|} 
See Ref. \\
9.2 \\
\end{tabular} & See Ref.9.2 \\
\hline $\begin{array}{l}\text { In-cell demineralized } \\
\text { water }\end{array}$ & $\begin{array}{l}\text { CUB 4, 5, 6, or } \\
7\end{array}$ & Previously discussed in Reference 10.1.1.b above. & 10.1.3.b & LOW & 3,4 \\
\hline Tank T-66 & TK PIT 6 & Miscellaneous solution collection tank. Discussed in Reference 10.2.1 below. & 10.1.3.c & \begin{tabular}{|l|} 
See Ref. \\
10.2 .1 \\
\end{tabular} & See Ref. 10.2.1 \\
\hline Tank T-79 & TK PIT 7 & Miscellaneous solution collection tank. Discussed in Reference 10.2.2 below. & 10.1.3.c & \begin{tabular}{|l} 
See Ref. \\
10.2 .2 \\
\end{tabular} & See Ref. 10.2.2 \\
\hline
\end{tabular}




\begin{tabular}{|c|c|c|c|c|c|}
\hline ITEM & LOCATION & DESCRIPTION & Reference & IMPACT & COMMENTS \\
\hline Tank T-21 & TK PIT 2 & Previously discussed in References 2.2.3 above. & 10.1.3.d & \begin{tabular}{|l|} 
See Ref. \\
2.2 .3
\end{tabular} & See Ref. 2.2.3 \\
\hline Tank T-23 & TK PIT 2 & Previously discussed in References 2.3.4 above. & 10.1.3.d & \begin{tabular}{|l|} 
See Ref. \\
2.2 .4 \\
\end{tabular} & See Ref. 2.2 .4 \\
\hline F-115 waste & \begin{tabular}{|l|l|} 
WASTE \\
TANK PIT
\end{tabular} & Previously discussed in Reference 9.2 above. & 10.1.3.d & \begin{tabular}{|l|} 
See Ref. \\
9.2 \\
\end{tabular} & See Ref.9.2 \\
\hline Tank T-66 & TK PIT 6 & Nominal 62.9 L tank constructed of Zr with Zr probes located in Tank Pit 6. & 10.2.1.a & HIGH & 11,12 \\
\hline $\begin{array}{l}\text { T-66 LL, DR, and PR } \\
\text { instr. }\end{array}$ & CR, MUA & $\begin{array}{l}\text { Foxboro pneumatic instrument is located in the CR and the DP cell and associated } \\
\text { polyethylene tubing is located in the MUA. The LL, DR, and PR lines are constructed of Zr } \\
\text { and originate in T-66 in Tank Pit 6, travel through the LAA, and are tied to the } \\
\text { polyethylene tubing that goes to the DP transmitters in the MUA. }\end{array}$ & 10.2.1.b & HIGH & $9,10,11,12$ \\
\hline T-66 sparger & $\begin{array}{l}\text { MUA, CR, TK } \\
\text { PIT } 6\end{array}$ & $\begin{array}{l}\text { There's a push button switch in CR to activate a solenoid in the MUA. An in-line orifice } \\
\text { controls air flow into the tank. }\end{array}$ & 10.2.1.c & LOW & $1,2,4$ \\
\hline $\begin{array}{l}\text { T-66 process and } \\
\text { instrument lines }\end{array}$ & TK PIT 6 & Lines are constructed of $\mathrm{Zr}$ and provide solution transfer routes into and out of T-66. & 10.2.1.d & HIGH & $9,10,11,12$ \\
\hline $\begin{array}{l}\text { T-66 vacuum } \\
\text { accumulator }\end{array}$ & CUB 6 & $\begin{array}{l}3 \text { in. diameter } \times 6 \text { in. long flanged glass pipe ( } \sim 1 \mathrm{~L} \text { volume) with specially fabricated } \mathrm{Zr} \text { top } \\
\text { and bottom plates. }\end{array}$ & 10.2.1.e & NCBU & N/A \\
\hline SA-T66 needle block & CUB 6 & Specially fabricated Zr block with two needles for pulling samples. & 10.2.1.e & NCBU & N/A \\
\hline $\begin{array}{l}\text { Auxiliary glass sample } \\
\text { flask }\end{array}$ & CUB 6 & $\begin{array}{l}1 \text { L glass bottle with 2-line stopper outfitted with polyethylene tubing connected to SA-T66 } \\
\text { position on CUB } 6 \text { sample rack for sampling T-66 and for connection to vacuum source. }\end{array}$ & 10.2.1.e & LOW & 1,2 \\
\hline $\begin{array}{l}\text { SA-T66 special 1-line } \\
\text { block }\end{array}$ & CUB 6 & $\begin{array}{l}\text { Special 1-line polyethylene block with 3/8 in. metal tubing on one side installed at SA-T66 } \\
\text { position on CUB } 6 \text { sample rack for sampling T-66 using auxiliary glass sample flask. }\end{array}$ & 10.2.1.e & LOW & 1,2 \\
\hline Tank T-79 & TK PIT 7 & Nominal 68.9 L tank constructed of HC with Zr probes located in Tank Pit 7. & 10.2.2.a & HIGH & 11,12 \\
\hline $\begin{array}{l}\text { T-79 LL, DR, and PR } \\
\text { instr. }\end{array}$ & CR, MUA & $\begin{array}{l}\text { Foxboro pneumatic instrument is located in the CR and the DP cell and associated } \\
\text { polyethylene tubing is located in the MUA. The LL, DR, and PR lines are constructed of Zr } \\
\text { and originate in T-79 in Tank Pit 7, travel through the LAA, and are tied to the } \\
\text { polyethylene tubing that goes to the DP transmitters in the MUA. }\end{array}$ & 10.2.2.b & HIGH & $9,10,11,12$ \\
\hline $\begin{array}{l}\text { T-79 vacuum } \\
\text { accumulator }\end{array}$ & CUB 7 & $\begin{array}{l}3 \text { in. diameter } \times 6 \text { in. long flanged glass pipe ( } \sim 1 \text { L volume) with specially fabricated } \mathrm{Zr} \text { top } \\
\text { and bottom plates. }\end{array}$ & 10.2.2.e & NCBU & N/A \\
\hline SA-T79 needle block & CUB 7 & Specially fabricated Zr block with two needles for pulling samples. & 10.2.2.e & NCBU & N/A \\
\hline $\begin{array}{l}\text { Auxiliary glass sample } \\
\text { flask }\end{array}$ & CUB 7 & $\begin{array}{l}1 \text { L glass bottle with 2-line stopper outfitted with polyethylene tubing connected to SA-T79 } \\
\text { position on CUB } 7 \text { sample rack for sampling T-79 and for connection to vacuum source. }\end{array}$ & 10.2.2.e & LOW & 1,2 \\
\hline $\begin{array}{l}\text { SA-T79 special 1-line } \\
\text { block }\end{array}$ & CUB 7 & $\begin{array}{l}\text { Special 1-line polyethylene block with } 3 / 8 \text { in. metal tubing on one side installed at SA-T79 } \\
\text { position on CUB } 7 \text { sample rack for sampling T-79using auxiliary glass sample flask. }\end{array}$ & 10.2.2.e & LOW & 1,2 \\
\hline
\end{tabular}


11.0 Miscellaneous CLEANEX Operations

\begin{tabular}{|c|c|c|c|c|c|}
\hline ITEM & LOCATION & DESCRIPTION & Reference & IMPACT & COMMENTS \\
\hline Tank M-1 & MUA & Previously discussed in Reference 1.2.1 above. & 11.1 .1 & LOW & $1,2,3,4$ \\
\hline Tank M-2 & MUA & \begin{tabular}{|l|l} 
Previously discussed in Reference 1.2.2 above. \\
\end{tabular} & 11.1 .1 & LOW & $1,2,3,4$ \\
\hline Tank M-3 & MUA & Previously discussed in Reference 2.1.2 above. & 11.1 .2 & MED & $8,9,10$ \\
\hline \begin{tabular}{|l|} 
Tank M-703 \\
\end{tabular} & MUA & Previously discussed in Reference 2.1.3 above. & 11.1 .3 & LOW & $1,2,3,4$ \\
\hline Tank M-70 & MUA & Previously discussed in Reference 2.1.4 above. & 11.1 .4 & LOW & $1,2,3,4$ \\
\hline Tank M-71 & MUA & Previously discussed in Reference 2.1.5 above. & 11.1 .5 & LOW & $1,2,3,4$ \\
\hline \begin{tabular}{|l|} 
Tank M-704 \\
\end{tabular} & MUA & Previously discussed in Reference 1.4.2 above. & 11.2 .1 & LOW & $1,2,3,4$ \\
\hline Tank M-706 & MUA & Previously discussed in Reference 2.2.3 above. & 11.2 .1 & LOW & $1,2,3,4$ \\
\hline Tank T-23 & $\begin{array}{l}\text { CUB 4, TK } \\
\text { PIT } 2\end{array}$ & Used for CLEANEX operations. Previously discussed in Reference 2.1.5 above. & 11.2.2.a & \begin{tabular}{|l|} 
See Ref. \\
2.3 .4 \\
\end{tabular} & See Ref. 2.3 .4 \\
\hline Tank M-71 & MUA & Used to add organic extractant to T-23. Previously discussed in Reference 2.1.5 above. & 11.2.2.a & LOW & $1,2,3,4$ \\
\hline Tank M-704 & MUA & Previously discussed in Reference 1.4.2 above. & 11.2.2.a & LOW & $1,2,3,4$ \\
\hline \begin{tabular}{|l|} 
Tank M-706 \\
\end{tabular} & MUA & Previously discussed in Reference 2.2.3 above. & 11.2.2.a & LOW & $1,2,3,4$ \\
\hline T-23 sampling & CUB 4 & Previously discussed in Reference 2.3.4.f above. & 11.2.2.b & LOW & $1,2,3,4$ \\
\hline T2302 GN & & $\begin{array}{l}\text { Standard Zr GN in Cubicle } 4 \text { right DC well, uptake from T-23 with polyethylene line } \\
\text { connected to C4C601 GN. }\end{array}$ & 11.2.3.a & LOW & 1,2 \\
\hline C4C601 GN & CUB 4 & $\begin{array}{l}\text { Standard GN on BR-4 to C4C601 jumper between Cubicle } 6 \text { and Cubicle } 4 \text { with } \\
\text { polyethylene line connected to T2302 GN. Previously discussed in Reference 3.6.2.k } \\
\text { above. }\end{array}$ & 11.2.3.b & LOW & $1,2,4$ \\
\hline C4C601 jumper & CUB 4 & $\begin{array}{l}\text { Hard piped jumper between Cubicle } 4 \text { left DC well to BR-4 Previously discussed in } \\
\text { Reference 3.6.2.j above. }\end{array}$ & 11.2.3.c & LOW & $1,2,4$ \\
\hline C4C601 jumper & $\begin{array}{l}\text { TK PIT 4, 5, } \\
\text { and } 6\end{array}$ & $\begin{array}{l}\text { Hard piped jumper between Cubicle } 6 \text { and Cubicle } 4 \text { Previously discussed in Reference } \\
\text { 3.6.2.i above. }\end{array}$ & 11.2.3.d & LOW & $1,2,4$ \\
\hline C4C601 GN & CUB 6 & $\begin{array}{l}\text { Gooseneck in Cubicle } 6 \text { left DC well to C4C601 jumper between Cubicle } 6 \text { and Cubicle } 4 \\
\text { with polyethylene line connected to T60702 GN Previously discussed in Reference 3.6.2.h } \\
\text { above. }\end{array}$ & 11.2.3.e & LOW & $1,2.4$ \\
\hline T60702 GN & CUB 6 & \begin{tabular}{|l} 
Standard Zr GN on BR-6 with polyethylene line connected to C4C601 GN Previously \\
discussed in Reference 9.2.8.b above. \\
\end{tabular} & 11.2.3.f & LOW & 1,2 \\
\hline PS-607 & CUB 7 & Previously discussed in Reference 9.2.8.d above. & 11.2.8.g & LOW & 1,2 \\
\hline T-607 & CUB 6 & Previously discussed in Reference 9.2.8.e above. & 11.2.3.h & MED & $3,4,5,6$ \\
\hline
\end{tabular}




\begin{tabular}{|c|c|c|c|c|c|}
\hline ITEM & LOCATION & DESCRIPTION & Reference & IMPACT & COMMENTS \\
\hline HV-607 & CUB 6 & Previously discussed in Reference 9.2.8.f above. & 11.2.3.i & LOW & 1,2 \\
\hline T60703 GN & CUB 6 & $\begin{array}{l}\text { Gooseneck on BR-6 with polyethylene line connected to T2103 G. Previously discussed in } \\
\text { Reference 9.2.8.g above. }\end{array}$ & 11.2.3.j & LOW & 1,2 \\
\hline T2103 & CUB 6 & $\begin{array}{l}\text { Standard Zr GN in Cubicle } 6 \text { right DC well with polyethylene line connected to T60703 } \\
\text { GN. }\end{array}$ & 11.2.3.k & LOW & 1,2 \\
\hline C7403 GN & CUB 7 & $\begin{array}{l}\text { Standard Zr GN in Cubicle } 7 \text { left DC well with polyethylene plug installed (teed to T2103 } \\
\text { line). }\end{array}$ & 11.2.3.l & LOW & 1,2 \\
\hline KP-607 & CUB 6 & Previously discussed in Reference 9.2.8.k. & 11.2.3.m & LOW & 1,2 \\
\hline Cubicle 6 vacuum & CUB 6 & Cubicle 6 Air Jet (C6AJ) for vacuum supply. Previously discussed in Reference 9.2.8.l. & 11.2.3.n & LOW & $1,2,3$ \\
\hline Miscellaneous supplies & $\begin{array}{l}\text { CR, MUA, } \\
\text { CUB 4,6,7 }\end{array}$ & Previously discussed in Reference 1.4.1.q above. & 11.2.3.0 & LOW & $1,2,3$ \\
\hline Tank M-70 (scrub add.) & MUA & Previously discussed in Reference 2.1.4 above. & 11.2.4.a & LOW & $1,2,3,4$ \\
\hline T-23 scrub sampling & CUB 4 & Previously discussed in Reference 2.3.4.e above. & 11.2.4.b & LOW & 1,2 \\
\hline $\begin{array}{l}\text { Scrub transfer from T- } \\
23 \text { to T-21 }\end{array}$ & $\begin{array}{l}\text { CUB 6, TK } \\
\text { PIT 2, TK PIT } \\
6 \\
\end{array}$ & Previously discussed in Reference 11.2.3 above. & 11.2.4.c & $\begin{array}{l}\text { See Ref. } \\
11.2 .3\end{array}$ & See Ref. 11.2.3 \\
\hline $\begin{array}{l}\text { Transfer of organic } \\
\text { from T-23 to T-60 }\end{array}$ & $\begin{array}{l}\text { CUB 6, TK } \\
\text { PIT 2, TK PIT } \\
6 \\
\end{array}$ & Previously discussed in Reference 11.2.3 above. & 11.2.4.d & $\begin{array}{l}\text { See Ref. } \\
11.2 .3 \text {, } \\
11.2 .4 . d \\
\end{array}$ & $\begin{array}{l}\text { See Ref. } \\
11.2 .3,11.2 .4 . \mathrm{d}\end{array}$ \\
\hline T6007 GN & CUB 6 & Standard Ta GN in Cubicle 6 left DC well with polyethylene line connected to T60703 GN. & 11.2.4.d & LOW & 1,2 \\
\hline $\begin{array}{l}\text { Scrub Transfer from T- } \\
23 \text { to T-60 }\end{array}$ & $\begin{array}{l}\text { CUB 6, TK } \\
\text { PIT 2, TK PIT } \\
6 \\
\end{array}$ & Previously discussed in Reference 11.2.3 and 11.2.4.d above. & 11.2.4.e & $\begin{array}{l}\text { See Ref. } \\
11.2 .5\end{array}$ & See Ref. 11.2.5 \\
\hline T-60 scrub sampling & CUB 6 & Previously discussed in Reference 6.2.1.e above. & 11.2.4.f & LOW & 1,2 \\
\hline $\begin{array}{l}\text { Scrub transfer from T- } \\
60 \text { to T-21 }\end{array}$ & $\begin{array}{l}\text { CUB } 6 \text { and } \\
\text { CUB } 7\end{array}$ & Discussed in Reference 11.2.5 below. & 11.2.4.g & \begin{tabular}{|l|} 
See Ref. \\
11.2 .5 \\
\end{tabular} & See Ref. 11.2 .5 \\
\hline T6002 GN & CUB 6 & $\begin{array}{l}\text { Standard Ta GN in Cubicle } 6 \text { right DC well with polyethylene line connected to T73402 } \\
\text { GN in Cubicle } 7 .\end{array}$ & 11.2.5.a & LOW & 1,2 \\
\hline T73402 GN & CUB 7 & $\begin{array}{l}\text { Previously discussed in Reference 1.6.1.d above with polyethylene line connected to } \\
\text { T6002 GN in Cubicle 6. }\end{array}$ & 11.2.5.b & LOW & 1,2 \\
\hline PS-733 & CUB 7 & Previously discussed in Reference 1.6.1.e above. & 11.2.5.c & LOW & 1,2 \\
\hline HV-T734-2 & CUB 7 & Previously discussed in Reference 1.6.1.f above. & 11.2.5.d & LOW & 1,2 \\
\hline
\end{tabular}




\begin{tabular}{|c|c|c|c|c|c|}
\hline ITEM & LOCATION & DESCRIPTION & Reference & IMPACT & COMMENTS \\
\hline Tank T-734 & CUB 7 & Previously discussed in Reference 1.6.1.g above. & 11.2.5.e & MED & 3,4 \\
\hline HV-T734-3 & CUB 7 & Previously discussed in Reference 1.6.1.h above. & 11.2.5.f & LOW & 1,2 \\
\hline HV-T734-1 & CUB 7 & Previously discussed in Reference 1.6.1.k above. & 11.2.5.g & LOW & 1,2 \\
\hline T73404 GN & CUB 7 & $\begin{array}{l}\text { Previously discussed in Reference 1.6.1.i above with polyethylene tubing connected to } \\
\text { C7403 GN. }\end{array}$ & 11.2.5.h & LOW & 1,2 \\
\hline C7403 GN & CUB 7 & $\begin{array}{l}\text { Standard Zr GN in Cubicle } 7 \text { left DC well with polyethylene line connected to T73404 GN } \\
\text { in Cubicle } 7 \text { (tees with T2103 line). }\end{array}$ & 11.2.5.i & LOW & 1,2 \\
\hline T2103 GN & CUB 6 & $\begin{array}{l}\text { Standard Zr GN in Cubicle } 6 \text { right DC well with polyethylene plug installed (tees with } \\
\text { C7403 line). }\end{array}$ & 11.2.5.j & LOW & 1,2 \\
\hline KP-735 & CUB 7 & Previously discussed in Reference 1.6.1.I above & 11.2.5.k & LOW & 1,2 \\
\hline CU7AJ & CUB 7 & Previously discussed in Reference 1.4.1.p above. & 11.2.5.1 & LOW & $1,2,4$ \\
\hline Miscellaneous supplies & $\begin{array}{l}\text { CR, MUA, } \\
\text { CUB 6,7 }\end{array}$ & Previously discussed in Reference 1.4.1.q above. & 11.2.5.m & LOW & 1,2 \\
\hline Tank M-71 & MUA & $\begin{array}{l}\text { Make up and addition of } 0.2 \mathrm{M} \text { Alamine } 336 \text { in Exxon Aromatic } 150 \text { (Phase Modifier) to T- } \\
60 \text { from M-71. Previously discussed in References 2.1.5 and 11.2.4.b above. }\end{array}$ & 11.2.6.a & LOW & $1,2,3,4$ \\
\hline Tank T-60 & $\begin{array}{l}\text { CR, MUA, } \\
\text { CUB 6,TK PIT } \\
6\end{array}$ & $\begin{array}{l}\text { Addition of Phase Modifier toT-60. Previously discussed in References 6.2.1, 6.2.2 and } \\
\text { 1.2.4.b above. }\end{array}$ & 11.2.6.a & \begin{tabular}{|l} 
See Ref. \\
6.2.1, \\
6.2.2, \\
11.2.4.b
\end{tabular} & $\begin{array}{l}\text { See Ref. 6.2.1, } \\
6.2 .2,11.2 .4 . b\end{array}$ \\
\hline Tank M-70 (strip add.) & MUA & Previously discussed in References 2.1.4 and 11.2.4.a above. & 11.2.6.b & LOW & $1,2,3,4$ \\
\hline T-60 strip sampling & CUB 6 & Previously discussed in Reference 6.2.1.e above. & 11.2.6.C & LOW & 1,2 \\
\hline T-60 organic sampling & CUB 6 & Previously discussed in Reference 6.2.1.e above. & 11.2.6.d & LOW & 1,2 \\
\hline T6002 GN & CUB 6 & Previously discussed in Reference 11.2.5.a above. & 11.2.7.a & LOW & 1,2 \\
\hline T73402 GN & CUB 7 & Previously discussed in Reference 1.6.1.d above. & 11.2.7.b & LOW & \begin{tabular}{|l|l|}
1,2 \\
1,2
\end{tabular} \\
\hline PS-733 & CUB 7 & Previously discussed in Reference 1.6.1.e above. & 11.2.7.c & LOW & 1,2 \\
\hline HV-T734-2 & CUB 7 & Previously discussed in Reference 1.6.1.f above. & 11.2.7.d & LOW & 1,2 \\
\hline Tank T-734 & CUB 7 & Previously discussed in Reference 1.6.1.g above. & 11.2.7.e & MED & 3,4 \\
\hline HV-T734-3 & CUB 7 & Previously discussed in Reference 1.6.1.h above. & 11.2.7.f & LOW & 1,2 \\
\hline T73404 GN & CUB 7 & $\begin{array}{l}\text { Previously discussed in Reference 1.6.1.i above with polyethylene tubing connected to } \\
\text { C7405 GN. }\end{array}$ & 11.2.7.g & LOW & 1,2 \\
\hline C7405 GN & CUB 7 & $\begin{array}{l}\text { Previously discussed in Reference } 2 \text {.3.7.h above with polyethylene line connected to } \\
\text { T73404 GN in Cubicle } 7 .\end{array}$ & 11.2.7.h & LOW & 1,2 \\
\hline HV-T734-1 & CUB 7 & Previously discussed in Reference 1.6.1.k above. & 11.2.7.i & LOW & 1,2 \\
\hline KP-735 & CUB 7 & Previously discussed in Reference 1.6.1.I above. & 11.2.7.j & LOW & 1,2 \\
\hline CU7AJ & CUB 7 & Previously discussed in Reference 1.4.1.p above. & 11.2.7.k & LOW & $1,2,4$ \\
\hline Miscellaneous supplies & $\begin{array}{l}\text { CR, MUA, } \\
\text { CUB 6,7 }\end{array}$ & Previously discussed in Reference 1.4.1.q above. & 11.2.7.l & LOW & 1,2 \\
\hline Tank T-604 & $\begin{array}{l}\text { CR, MUA, } \\
\text { CUB 6,TK PIT } \\
6 \\
\end{array}$ & Previously discussed in References 2.3.8 and 2.3.9 above. & 11.2 .8 & $\begin{array}{l}\text { See Ref. } \\
2.3 .8 \\
2.3 .9 \\
\end{array}$ & $\begin{array}{l}\text { See Ref. 2.3.8, } \\
2.3 .9\end{array}$ \\
\hline
\end{tabular}




\begin{tabular}{|c|c|c|c|c|c|}
\hline ITEM & LOCATION & DESCRIPTION & Reference & IMPACT & COMMENTS \\
\hline 7PDV-604-1 & CUB 6 & Previously discussed in Reference 2.3.10.a above. & $11.2 .9 \mathrm{a}$ & LOW & 1,2 \\
\hline PS-604 & CUB 6 & Previously discussed in Reference 2.3.10.b above. & 11.2.9.b & LOW & 1,2 \\
\hline HCV-P604-1 & CUB 6 & Previously discussed in Reference 2.3.10.c above. & 11.2.9.c & LOW & 1,2 \\
\hline P65301 GN & CUB 6 & Previously discussed in Reference $2.3 .10 . d$ above. & 11.2.9.d & LOW & 1,2 \\
\hline P-653 & CUB 6 & Previously discussed in Reference 2.3.10.e above. & 11.2.9.e & MED & $5,6,8$ \\
\hline P65302 GN & CUB 6 & Previously discussed in Reference 2.3.10.f above. & 11.2.9.f & LOW & 1,2 \\
\hline FDV-T604 & CUB 6 & Previously discussed in Reference 2.3.10.g above. & 11.2.9.g & LOW & 1,2 \\
\hline C4C601 GN & CUB 6 & Previously discussed in Reference 3.6.2.h above. & 11.2.9.h & LOW & 1,2 \\
\hline C4C601 jumper & $\begin{array}{l}\text { TK PIT 4, 5, } \\
\text { and } 6 \\
\end{array}$ & Previously discussed in Reference 3.6.2.i above. & 11.2.9.i & LOW & 1,2 \\
\hline C4C601 jumper & CUB 4 & Previously discussed in Reference 3.6.2.j above. & 11.2.9.j & LOW & 1,2 \\
\hline C4C601 GN & CUB 4 & Previously discussed in Reference 3.6.2.k above. & 11.2.9.k & LOW & 1,2 \\
\hline T4003 GN & CUB 4 & Previously discussed in Reference 3.6.2.I above. & 11.2.9.1 & LOW & 1,2 \\
\hline T4013 GN & CUB 6 & \begin{tabular}{|l} 
Previously discussed in Reference 3.6.2.m above. \\
\end{tabular} & 11.2.9.m & LOW & 1,2 \\
\hline 7PFDV-604-2 & CUB 6 & Previously discussed in Reference 2.3.10.h above. & 11.2.9.n & LOW & 1,2 \\
\hline HCV-P604-2 & CUB 6 & Previously discussed in Reference 2.3.10.i above. & 11.2.9.0 & LOW & 1,2 \\
\hline Miscellaneous supplies & $\begin{array}{l}\text { CR, MUA, } \\
\text { CUB 4, } 6 \\
\end{array}$ & Previously discussed in Reference 1.4.1.q above. & 11.2.9.p & LOW & 1,2 \\
\hline $\begin{array}{l}\text { Tank M-70 (confirming } \\
\text { strip add.) }\end{array}$ & MUA & Previously discussed in References 2.1.4 and 11.2.4.a above. & 11.2.10.a & LOW & $1,2,3,4$ \\
\hline $\begin{array}{l}\text { T-604 confirming strip } \\
\text { sampling }\end{array}$ & CUB 6 & Previously discussed in Reference 2.3.8.e above. & 11.2.10.b & LOW & 1,2 \\
\hline $\begin{array}{l}\text { Transfer of confirming } \\
\text { strip from T-604 to } \\
\text { T-40 }\end{array}$ & $\begin{array}{l}\text { CUB 4,CUB 6, } \\
\text { TK PIT 4, 5, } \\
\text { and } 6\end{array}$ & Previously discussed in Reference 11.2.9 above. & 11.2.10.c & \begin{tabular}{|l} 
See Ref. \\
11.2 .9
\end{tabular} & See Ref. 11.2.9 \\
\hline T-604 organic sampling & CUB 6 & Previously discussed in Reference 2.3.8.e above. & 11.2.10.d & LOW & 1,2 \\
\hline T-40 evaporation & $\begin{array}{l}\text { CR, MUA, TK } \\
\text { PIT } 4\end{array}$ & Previously discussed in References 9.2.11 and 9.2.12 above. & 11.2.10.e & $\begin{array}{l}\text { See Ref. } \\
9.2 .11 \\
9.2 .12 \\
\end{array}$ & $\begin{array}{l}\text { See Ref. } \\
9.2 .11,9.2 .12\end{array}$ \\
\hline T-40 sampling & CUB 4 & Previously discussed in Reference 9.2.11.e above. & 11.2.10.f & LOW & 1,2 \\
\hline $\begin{array}{l}\text { Tank T-60, NaOH } \\
\text { addition }\end{array}$ & \begin{tabular}{|l|} 
MUA,TK PIT \\
6 \\
\end{tabular} & Caustic addition to neutralize the acid heel and waste organic. & 11.2.11.a, & LOW & 1,2 \\
\hline $\begin{array}{l}\text { Tank T-604, NaOH } \\
\text { addition }\end{array}$ & \begin{tabular}{|l|} 
MUA,TK PIT \\
6
\end{tabular} & Caustic addition to neutralize the acid heel and waste organic. & 11.2.11.a, & LOW & 1,2 \\
\hline
\end{tabular}




\begin{tabular}{|c|c|c|c|c|c|}
\hline ITEM & LOCATION & DESCRIPTION & Reference & IMPACT & COMMENTS \\
\hline Tank T-60 sampling & TK PIT 6 & Previously discussed in Reference 6.2.1.e above. & 11.2.11.b & LOW & 1,2 \\
\hline Tank T-604 sampling & TK PIT 6 & Previously discussed in Reference 2.3.8.e above. & 11.2.11.b & LOW & 1,2 \\
\hline $\begin{array}{l}\text { T-60 and T-604 waste } \\
\text { organic transfers }\end{array}$ & $\begin{array}{l}\text { CUB 7, TK } \\
\text { PIT } 6\end{array}$ & Discussed in Reference 11.2.12 and Reference 11.2.13 below, respectively. & 1.2.11.c & \begin{tabular}{|l} 
See Ref. \\
11.2 .12$, \\
11.2 .13 \\
\end{tabular} & $\begin{array}{l}\text { See Ref. } \\
11.2 .12, \\
11.2 .13 \\
\end{array}$ \\
\hline $\begin{array}{l}\text { Tank M-703 transfer } \\
\text { system }\end{array}$ & MUA & Previously discussed in Reference 2.1.3 above. & 11.2.11.d & LOW & $1,2,3,4$ \\
\hline T-60 waste jet & \begin{tabular}{|l|} 
MUA, TK PIT \\
6
\end{tabular} & $\begin{array}{l}\text { T-60 waste jet (J-T60) to F-115. Previously discussed in Reference 9.2.15.e above or } \\
\text { Reference 11.2.12 if the jet does not work. }\end{array}$ & 11.2.11.e. & MED & 4,7 \\
\hline T-604 waste jet & $\begin{array}{l}\text { MUA, TK PIT } \\
6\end{array}$ & $\begin{array}{l}\text { T-604 waste jet (J-T604) to F-115. Previously discussed in Reference 9.2.15.e above or } \\
\text { Reference 11.2.13 if the jet does not work. }\end{array}$ & 11.2.11.e & MED & 4,7 \\
\hline T-60 evaporation & $\begin{array}{l}\text { CR, MUA, TK } \\
\text { PIT } 6\end{array}$ & Previously discussed in References 6.2.1 and 6.2.2 above. & 11.2.11.f & \begin{tabular}{|l} 
See Ref. \\
6.2.1, \\
6.2.2 \\
\end{tabular} & $\begin{array}{l}\text { See Ref. 6.2.1, } \\
6.2 .2\end{array}$ \\
\hline T-604 evaporation & $\begin{array}{l}\text { CR, MUA, TK } \\
\text { PIT } 6\end{array}$ & Previously discussed in References 2.3.8 and 2.3.9 above. & 11.2.11. f & \begin{tabular}{|l} 
See Ref. \\
2.3 .8 , \\
2.3 .8 \\
\end{tabular} & $\begin{array}{l}\text { See Ref. 2.3.8, } \\
2.3 .8\end{array}$ \\
\hline $\begin{array}{l}\text { T-60 and T-604 waste } \\
\text { jets }\end{array}$ & $\begin{array}{l}\text { MUA, TK } \\
\text { PIT6 }\end{array}$ & Previously discussed in Reference 9.2.15.e above. & 11.2.11.g & MED & 4,7 \\
\hline T6002 GN & CUB 6 & Previously discussed in Reference 11.2.5.a above. & 11.2.12.a & LOW & 1,2 \\
\hline T73402 GN & CUB 7 & Previously discussed in Reference 1.6.1.d above. & 11.2.12.b & LOW & 1,2 \\
\hline PS-733 & CUB 7 & Previously discussed in Reference 1.6.1.e above. & 11.2.12.c & LOW & 1,2 \\
\hline HV-T734-2 & CUB 7 & Previously discussed in Reference 1.6.1.f above. & 11.2.12.d & LOW & 1,2 \\
\hline Tank T-734 & CUB 7 & Previously discussed in Reference 1.6.1.g above. & 11.2.12.e & MED & 3,4 \\
\hline HV-T734-3 & CUB 7 & Previously discussed in Reference 1.6.1.h above. & 11.2.12.f & LOW & 1,2 \\
\hline HV-T734-1 & CUB 7 & Previously discussed in Reference 1.6.1.k above. & 11.2.12.g & LOW & 1,2 \\
\hline T73404 GN & CUB 7 & Previously discussed in Reference 1.6.1.i above. & 11.2.12.h & LOW & 1,2 \\
\hline $\begin{array}{l}\text { SA-F126 special 1-line } \\
\text { block }\end{array}$ & CUB 7 & Previously discussed in Reference 9.2.16.i above. & 11.2.12.i & LOW & 1,2 \\
\hline KP-735 & CUB 7 & Previously discussed in Reference 1.6.1.I above. & 11.2.12.j & LOW & 1,2 \\
\hline CU7AJ & CUB 7 & Previously discussed in Reference 1.4.1.p above. & $11.2 .12 . \mathrm{k}$ & LOW & 1,2 \\
\hline Miscellaneous supplies & $\begin{array}{l}\text { CR, MUA, } \\
\text { CUB 6,7 }\end{array}$ & Previously discussed in Reference 1.4.1.q above. & 11.2.12.l & LOW & 1,2 \\
\hline \begin{tabular}{|l|} 
7PDV-604-1 \\
\end{tabular} & CUB 6 & Previously discussed in Reference 2.3.10. above. & 11.2.13.a & LOW & 1,2 \\
\hline PS-604 position & CUB 6 & Previously discussed in Reference 2.3.17.b above. & 11.2.13.b & LOW & 1,2 \\
\hline T73402 GN & CUB 7 & Previously discussed in Reference 1.6.1.d above. & 11.2.13.c & LOW & 1,2 \\
\hline \begin{tabular}{|l|} 
PS-733 \\
\end{tabular} & CUB 7 & Previously discussed in Reference 1.6.1.e above. & 11.2.13.d & LOW & 1,2 \\
\hline HV-T734-2 & CUB 7 & Previously discussed in Reference 1.6.1.f above. & 11.2.12.e & LOW & 1,2 \\
\hline
\end{tabular}




\begin{tabular}{|c|c|c|c|c|c|}
\hline ITEM & LOCATION & DESCRIPTION & Reference & IMPACT & COMMENTS \\
\hline Tank T-734 & CUB 7 & Previously discussed in Reference 1.6.1.g above. & 11.2.13.f & MED & 3,4 \\
\hline HV-T734-3 & CUB 7 & Previously discussed in Reference 1.6.1.h above. & 11.2.13.g & LOW & 1,2 \\
\hline HV-T734-1 & CUB 7 & Previously discussed in Reference 1.6.1.k above. & 11.2.13.h & LOW & 1,2 \\
\hline T73404 GN & CUB 7 & Previously discussed in Reference 1.6.1.i above. & 11.2.13.i & LOW & 1,2 \\
\hline $\begin{array}{l}\text { SA-F126 special 1-line } \\
\text { block }\end{array}$ & CUB 7 & Previously discussed in Reference 9.2.16.i above. & 11.2.13.j & LOW & 1,2 \\
\hline KP-735 & CUB 7 & Previously discussed in Reference 1.6.1.I above. & 11.2.13.k & LOW & 1,2 \\
\hline CU7AJ & CUB 7 & Previously discussed in Reference 1.4.1.p above. & 11.2.13.1 & LOW & 1,2 \\
\hline Miscellaneous supplies & $\begin{array}{l}\text { CR, MUA, } \\
\text { CUB 6,7 }\end{array}$ & Previously discussed in Reference 1.4.1.q above. & $11.2 .13 . \mathrm{m}$ & LOW & 1,2 \\
\hline
\end{tabular}


12.0 BERKEX Operations

\begin{tabular}{|c|c|c|c|c|c|}
\hline ITEM & LOCATION & DESCRIPTION & Reference & IMPACT & COMMENTS \\
\hline Polyethylene bottles & MUA & $\begin{array}{l}\text { Standard } 1 \mathrm{~L} \text { and } 250 \mathrm{ml} \text { polyethylene bottles used for cold reagents, raffinates, and product } \\
\text { collection. }\end{array}$ & 12.1 .1 & LOW & 1,2 \\
\hline $\mathrm{NaBrO}_{3}$ & MUA & $2.0 \mathrm{M} \mathrm{NaBrO}_{3}$ is made up from stock $\mathrm{NaBrO}_{3}$ usually kept on hand for make ups. & 12.1 .2 & LOW & 1,2 \\
\hline $\mathrm{HNO}_{3}$ & MUA & 8.0 $\mathrm{M} \mathrm{HNO}_{3}$ is made up from stock concentrated $\mathrm{HNO}_{3}$ usually kept on hand for make ups. & 12.1 .3 & LOW & 1,2 \\
\hline HDEHP in dodecane & MUA & $\begin{array}{l}0.5 M \text { HDEHP in dodecane is made up from stock HDEHP and stock dodecane usually } \\
\text { kept on hand for make ups. }\end{array}$ & 12.1.4 & LOW & 1,2 \\
\hline $\mathrm{H}_{2} \mathrm{O}_{2}$ & MUA & $30 \% \mathrm{H}_{2} \mathrm{O}_{2}$ is poured up from the stock $\mathrm{H}_{2} \mathrm{O}_{2}$ solution usually kept on hand for make ups. & 12.1 .5 & LOW & 1,2 \\
\hline $\begin{array}{l}\text { Small volume glass } \\
\text { pipettes and rubber } \\
\text { bulbs/plastic pipettes }\end{array}$ & CUB 4 & $\begin{array}{l}\text { Approximately } 5 \mathrm{ml} \text { glass pipettes with rubber bulbs to fit the size of the pipette used } \\
\text { sampling solutions. Plastic pipettes are substituted for the glass pipettes and rubber bulbs } \\
\text { when warranted. }\end{array}$ & 12.1.6 & LOW & 1,2 \\
\hline Polyethylene bottles & MUA & $\begin{array}{l}\text { Standard } 1 \mathrm{~L} \text { and } 250 \mathrm{ml} \text { polyethylene bottles used for cold reagents, raffinates, and product } \\
\text { collection. Previously discussed in Reference 12.1.1 above. }\end{array}$ & 12.2.1.a & LOW & 1,2 \\
\hline Alpha instrumentation & CR, CUB 4 & $\begin{array}{l}\text { Specially fabricated detector for use in detecting alpha radiation in solutions. Installed at } \\
\text { RE-C401 position on RR-4. Previously discussed in Reference 3.4.9.a above. }\end{array}$ & 12.2.1.b & HIGH & 9,10 \\
\hline $\begin{array}{l}\text { Neutron } \\
\text { instrumentation }\end{array}$ & CR, CUB 4 & $\begin{array}{l}\text { Specially fabricated detector for use in detecting neutron radiation. Used for monitoring } \\
\text { radiation in solutions and equipment to track and evaluate process efficiency. Previously } \\
\text { discussed in Reference 3.4.9.b above. }\end{array}$ & 12.2.1.c & HIGH & 9,10 \\
\hline $\begin{array}{l}\text { Support equipment and } \\
\text { materials }\end{array}$ & CR, CUB 4 & Previously discussed in Reference 3.4.9.c and 3.4.9.d above. . & 12.2.1.d & HIGH & 9,10 \\
\hline $\mathrm{V}-13$ and $\mathrm{V}-14$ & CUB 4 & Previously discussed in Reference 4.2.3.i above. & 12.2.2.a & LOW & 1,2 \\
\hline $\begin{array}{l}\text { V-T408-1, V-T408-2, } \\
\text { V-T408-3, V-T404-1, } \\
\text { V-T404-2, V-T404-3, } \\
\text { and V-T404-4 }\end{array}$ & MUA & Previously discussed in Reference 4.2.3.n above. & 12.2.2.b & LOW & 1,2 \\
\hline PIC T404 & CR & Previously described in Section 4.2.3.k above. & 12.2.2.c & LOW & 1,2 \\
\hline PCV-T404 & MUA & Previously described in Section 4.2.3.I above. & 12.2.2.d & LOW & 1,2 \\
\hline
\end{tabular}




\begin{tabular}{|c|c|c|c|c|c|}
\hline ITEM & LOCATION & DESCRIPTION & Reference & IMPACT & COMMENTS \\
\hline PI-T404-2 & MUA & Previously described in Section 4.2.3.m above & 12.2.2.e & LOW & 1,2 \\
\hline Miscellaneous supplies & $\begin{array}{l}\text { CR, MUA, } \\
\text { CUB } 4\end{array}$ & $\begin{array}{l}\text { Miscellaneous equipment and supplies such as funnels, polyethylene bottles, polyethylene } \\
\text { tubing, bottle holders, etc. Previously discussed in Section 4.2.1.h above. }\end{array}$ & 12.2.2.f & LOW & 1,2 \\
\hline Tank T-404 & CUB 4 & Previously described in Section 4.2.3.g above. & 12.2.3.a & & \\
\hline $\begin{array}{l}1 / 4 \text { in. polyethylene } \\
\text { tubing with male ball } \\
\text { joint }\end{array}$ & CUB 4 & $\begin{array}{l}1 / 4 \text { in. diameter polyethylene tubing pre-made in house with male ball joint on one end } \\
\text { connected to female ball joint at V-15. }\end{array}$ & 12.2.3.b & LOW & 1,2 \\
\hline $\mathrm{V}-13$ and $\mathrm{V}-14$ & CUB 4 & Previously discussed in Reference 4.2.3.i above. & 12.2.3.c & LOW & 1,2 \\
\hline PI-T404 & CUB 4 & Previously described in Section 4.2.3.h above. & 12.2.3.d & LOW & 1,2 \\
\hline Cubicle 4 vacuum & CUB 4 & $\begin{array}{l}\text { Cubicle } 4 \text { Air Jet (CU4AJ) for vacuum supply. Previously described in Section 4.2.3.j } \\
\text { above. }\end{array}$ & 12.2.3.e & LOW & 1,2 \\
\hline RE-401 & CUB 4 & Previously described in Section 4.2.1.f above. & 12.2.3.f & LOW & 1,2 \\
\hline PIC T404 & CR & Previously described in Section 4.2.3.k above. & 12.2.3.g & LOW & 1,2 \\
\hline PCV-T404 & MUA & Previously described in Section 4.2.3.I above. & 12.2.3.h & LOW & 1,2 \\
\hline PI-T404-2 & MUA & Previously described in Section 4.2.3.m above. & 12.2.3.i & LOW & 1,2 \\
\hline $\begin{array}{l}\text { V-T408-1, V-T408-2, } \\
\text { V-T408-3, V-T404-1, } \\
\text { V-T404-2, V-T404-3, } \\
\text { and V-T404-4 }\end{array}$ & MUA & Previously discussed in Reference 4.2.3.n above. & 12.2.3.j & LOW & 1,2 \\
\hline Miscellaneous supplies & $\begin{array}{l}\text { CR, MUA, } \\
\text { CUB } 4\end{array}$ & $\begin{array}{l}\text { Miscellaneous equipment and supplies such as funnels, polyethylene bottles, polyethylene } \\
\text { tubing, bottle holders, etc. Previously discussed in Section 4.2.1.h above. }\end{array}$ & 12.2.3.k & LOW & 1,2 \\
\hline $\begin{array}{l}\text { Bk feed loading of C- } \\
402\end{array}$ & $\begin{array}{l}\text { CR, MUA, } \\
\text { CUB } 4 \\
\end{array}$ & Previously discussed in Reference 12.2.3 above. & 12.2.4.a & \begin{tabular}{|l|} 
See Ref. \\
12.2 .3 \\
\end{tabular} & See Ref. 12.2.3 \\
\hline Raffinate collection & CUB 4 & $\begin{array}{l}\text { Raffinate is collected in labeled and calibrated } 1 \mathrm{~L} \text { polyethylene bottle. Previously } \\
\text { discussed in Reference 12.1.1 above. }\end{array}$ & 12.2.4.b & LOW & 1,2 \\
\hline Column flush & $\begin{array}{l}\text { CR, MUA, } \\
\text { CUB } 4\end{array}$ & Previously discussed in Reference 12.2.3 above. & 12.2.4.c & \begin{tabular}{|l|} 
See Ref. \\
12.2 .3 \\
\end{tabular} & See Ref. 12.2 .3 \\
\hline Flush collection & CUB 4 & $\begin{array}{l}\text { Flushes are collected in a labeled and calibrated } 1 \text { L polyethylene bottle. Previously } \\
\text { discussed in Reference } \mathbf{1 2 . 1 . 1} \text { above. }\end{array}$ & 12.2.4.b & LOW & 1,2 \\
\hline Bk stripping of C-402 & $\begin{array}{l}\text { CR, MUA, } \\
\text { CUB } 4\end{array}$ & Previously discussed in Reference 12.2.3 above. & 12.2.5.a & \begin{tabular}{|l} 
See Ref. \\
12.2 .3 \\
\end{tabular} & See Ref. 12.2.3 \\
\hline
\end{tabular}




\begin{tabular}{|c|c|c|c|c|c|}
\hline ITEM & LOCATION & DESCRIPTION & Reference & IMPACT & COMMENTS \\
\hline $\begin{array}{l}\text { Strip (Bk Product) } \\
\text { collection }\end{array}$ & CUB 4 & $\begin{array}{l}\text { Strips are collected in a labeled and calibrated } 1 \mathrm{~L} \text { polyethylene bottle. Previously discussed } \\
\text { in Reference 12.1.1 above. }\end{array}$ & 12.2.5.b & LOW & 1,2 \\
\hline Column flush & $\begin{array}{l}\text { CR, MUA, } \\
\text { CUB } 4 \\
\end{array}$ & Previously discussed in Reference 12.2.3 above. & 12.2.5.c & \begin{tabular}{|l|} 
See Ref. \\
12.2 .3 \\
\end{tabular} & See Ref. 12.2.3 \\
\hline Flush collection & CUB 4 & $\begin{array}{l}\text { Flushes are collected in labeled and calibrated } 1 \mathrm{~L} \text { polyethylene bottles (Previously } \\
\text { discussed in Reference 12.1.1 above.). }\end{array}$ & 12.2.5.d & LOW & 1,2 \\
\hline $\begin{array}{l}\text { Acidification of } \\
\text { raffinates and flushes }\end{array}$ & CUB 4, MUA & Concentrated $\mathrm{HNO}_{3}$ is used for acidification. & 12.2.6.a & LOW & 1,2 \\
\hline Sampling equipment & CUB 4 & Previously discussed in Reference 12.1.6 above. & 12.2.6.b & LOW & 1,2 \\
\hline V-25 (pinch clamp) & CUB 4 & A standard type laboratory pinch clamp for rubber tubing mounted on RR-4. & 12.3.1.a & LOW & 1,2 \\
\hline Tank T-411 & CUB 4 & $\begin{array}{l}\text { Bk extraction pot, } \sim 4.5 \text { L glass tank located on RR-4 with drain line off the bottom of the } \\
\text { tank. }\end{array}$ & 12.3.1.b & MED & $5,6,11,14$ \\
\hline $\begin{array}{l}1 / 2 \text {-in JACO with } 1 / 2 \text {-in } \\
\text { polyethylene line with } \\
\text { female ball joint }\end{array}$ & CUB 4 & Specially fabricated line and JACO combination to fit onto the drain of Tank T-411. & 12.3.1.c & LOW & 1,2 \\
\hline Phase separator & CUB 4 & $\begin{array}{l}\text { Specially fabricated equipment for performing phase separations of organic and aqueous } \\
\text { solutions. Fabricated using a } 25 \mathrm{ml} \text { volumetric glass pipette with Tygon type tubing } \\
\text { connected to the top of the pipette and a male ball joint on the other end of the Tygon } \\
\text { tubing. }\end{array}$ & 12.3.1.d & LOW & 1,2 \\
\hline Funnel & CUB 4 & Special funnel with adapter for connection to T4503 GN in Cubicle 4 right DC well. & 12.3.1.e & LOW & 1,2 \\
\hline Tank T-409 & CUB 4 & Vacuum KOP, 4.5 L glass tank located on RR-4 with dip line into tank. & 12.3.1.f & MED & $5,6,11,14$ \\
\hline $\begin{array}{l}\text { Special section of } \\
3 / 8 \text { in. polyethylene } \\
\text { tubing }\end{array}$ & CUB 4 & $\begin{array}{l}\text { Specially fabricated section of } 3 / 8 \text { in. polyethylene tubing heated and formed into shape for } \\
\text { use as a vacuum extension for T-409. A male ball joint is on the short end and a female ball } \\
\text { joint is on the long end. }\end{array}$ & 12.3.1.g & LOW & 1,2 \\
\hline $\begin{array}{l}\text { Special section of } \\
3 / 8 \text { in. polyethylene } \\
\text { tubing }\end{array}$ & CUB 4 & $\begin{array}{l}\text { Specially fabricated section of } 3 / 8 \text { in. polyethylene tubing heated and formed into shape for } \\
\text { use as a vacuum source from T- } 409 \text { dip line to T- } 411 \text {. Male ball joints are on both ends. }\end{array}$ & 12.3.1.h & LOW & 1,2 \\
\hline $1 \mathrm{~L}$ polyethylene bottle & CUB 4 & Used for collection of scrub raffinate. Previously discussed in Reference 12.1.1 above. & 12.3.1.i & LOW & 1,2 \\
\hline $2.0 \mathrm{M} \mathrm{NaBrO}_{3}$ & CUB 4 & Previously discussed in Reference 12.1.2 above. & 12.3.1.j & LOW & 1,2 \\
\hline
\end{tabular}




\begin{tabular}{|c|c|c|c|c|c|}
\hline ITEM & LOCATION & DESCRIPTION & Reference & IMPACT & COMMENTS \\
\hline Concentrated $\mathrm{HNO}_{3}$ & CUB 4 & Used for acidification of solutions. & 12.3.1.k & LOW & 1,2 \\
\hline $\begin{array}{l}\text { Miscellaneous } \\
\text { calibrated } 250 \mathrm{ml} \\
\text { polyethylene bottle }\end{array}$ & CUB 4 & Previously discussed in Reference 12.1.1 above. & 12.3.1.1 & LOW & 1,2 \\
\hline Hot feed & CUB 4 & $600 \mathrm{ml}$ of solution prepared from operations in Reference 12.2.1-12.2.6 above. & 12.3.1.m & LOW & 1,2 \\
\hline $\begin{array}{l}0.5 M \text { HDEHP in } \\
\text { dodecane }\end{array}$ & CUB 4 & $500 \mathrm{ml}$ of organic extractant made up per Reference 12.1.4 above. & 12.3.1.n & LOW & 1,2 \\
\hline $8 \mathrm{M} \mathrm{HNO}_{3}$ & CUB 4 & $8 \mathrm{M} \mathrm{HNO}_{3}$ made up per Reference 12.1.3 above. & 12.3.1.o & LOW & 1,2 \\
\hline $\begin{array}{l}\text { Specially sleeved } 1 \mathrm{~L} \\
\text { polyethylene bottle for } \\
\text { the Bk product }\end{array}$ & CUB 4 & $\begin{array}{l}\text { A calibrated and labelled } 1 \mathrm{~L} \text { polyethylene bottle used to collect the Bk Product. The bottle } \\
\text { is specially sleeved with polyethylene sleeving to keep it as clean as possible for removal } \\
\text { from the hot cell bank. }\end{array}$ & 12.3.1.p & LOW & 1,2 \\
\hline $8 \mathrm{M} \mathrm{HNO}_{3}$ & CUB 4 & $8 \mathrm{M} \mathrm{HNO}_{3}$ made up per Reference 12.1.3 above. & 12.3.1.q & LOW & 1,2 \\
\hline $2.0 \mathrm{M} \mathrm{NaBrO}_{3}$ & CUB 4 & Previously discussed in Reference 12.1.2 above. & 12.3.1.r & LOW & 1,2 \\
\hline $30 \% \mathrm{H}_{2} \mathrm{O}_{2}$ & MUA & Previously discussed in Reference 12.1.5 above. & 12.3.1.s & LOW & 1,2 \\
\hline V-25 (pinch clamp) & CUB 4 & Previously discussed in Reference 12.3.1 a above. & 12.3.2.a & LOW & 1,2 \\
\hline Tank T-411 & CUB 4 & Previously discussed in Reference 12.3.1 b above. & 12.3.2.b & MED & $5,6,11,14$ \\
\hline Cubicle 4 vacuum & CUB 4 & Previously described in Section 4.2.3.j above. & 12.3.2.c & LOW & 1,2 \\
\hline Funnel & CUB 4 & Previously discussed in Reference 12.3.1 e above. & 12.3.2.d & LOW & 1,2 \\
\hline Tank T-409 & CUB 4 & Previously discussed in Reference 12.3.1 f above. & 12.3.2.e & MED & $5,6,11,14$ \\
\hline $\begin{array}{l}\text { Special section of } \\
3 / 8 \text { in. polyethylene } \\
\text { tubing }\end{array}$ & CUB 4 & Previously discussed in Reference 12.3.1 g above. & 12.3.2.f & LOW & 1,2 \\
\hline $\begin{array}{l}\text { Special section of } \\
3 / 8 \text { in. polyethylene } \\
\text { tubing }\end{array}$ & CUB 4 & Previously discussed in Reference 12.3.1 h above. & 12.3.2.g & LOW & 1,2 \\
\hline $\mathrm{V}-23$ & CUB 4 & $\begin{array}{l}\text { Hoke }{ }^{\circledR} \text { brand ball valve, modified specifically for installation and use on RR-4. This is the } \\
\text { vent vale on T- } 411 \text { and is currently in the open position with a broken handle. }\end{array}$ & 12.3.2.h & LOW & 1,2 \\
\hline Male ball joint plug & CUB 4 & Installed on the line to V-23 for T-411. & 12.3.2.i & LOW & 1,2 \\
\hline Vacuum gauge & CUB 4 & Installed on vacuum source in Cubicle 4. & 12.3.2.j & LOW & 1,2 \\
\hline $\begin{array}{l}\text { Wash solution transfer } \\
\text { into T-411 }\end{array}$ & CUB 4 & Transferred into T-411 per the route in Reference 12.3.2 above. & 12.4 .1 & $\begin{array}{l}\text { See Ref. } \\
12.3 .2\end{array}$ & See Ref. 12.3 .2 \\
\hline
\end{tabular}




\begin{tabular}{|c|c|c|c|c|c|}
\hline ITEM & LOCATION & DESCRIPTION & Reference & IMPACT & COMMENTS \\
\hline $\begin{array}{l}\text { Wash solution transfer } \\
\text { out of T-411 }\end{array}$ & CUB 4 & Transferred out of T-411 into T-45 per the route in Reference 12.3.2 above. & 12.4 .2 & \begin{tabular}{|l|} 
See Ref. \\
12.3 .2
\end{tabular} & See Ref. 12.3 .2 \\
\hline \begin{tabular}{|l|} 
Hot feed transfer into \\
T-411
\end{tabular} & CUB 4 & Transferred into T-411 per the route in Reference 12.3.2 above. & 12.5 .1 & \begin{tabular}{|l|} 
See Ref. \\
12.3 .2 \\
\end{tabular} & See Ref. 12.3 .2 \\
\hline \begin{tabular}{|l|}
$2.0 \mathrm{M} \mathrm{NaBrO}_{3}$ \\
TRANSFER into T-411 \\
\end{tabular} & CUB 4 & Transferred into T-411 per the route in Reference 12.3.2 above. & 12.5 .2 & \begin{tabular}{|l|} 
See Ref. \\
12.3 .2 \\
\end{tabular} & See Ref. 12.3 .2 \\
\hline $\begin{array}{l}0.5 M \text { HDEHP in } \\
\text { dodecane transfer into } \\
\text { T-411 }\end{array}$ & CUB 4 & Transferred into T-411 per the route in Reference 12.3.2 above. & 12.5 .3 & \begin{tabular}{|l} 
See Ref. \\
12.3 .2
\end{tabular} & See Ref. 12.3 .2 \\
\hline Mix in T-411 & CUB 4 & $\begin{array}{l}\text { While pulling vacuum on T-411, the pinch valve, V-25, is left slightly to achieve the } \\
\text { desired mixing in T-411. }\end{array}$ & 12.5 .4 & LOW & 1,2 \\
\hline Settle in T-411 & CUB 4 & $\begin{array}{l}\text { The pinch valve, V-25, is closed and the vacuum turned off of T-411. Reference Section } \\
\text { 12.3.1.a above. }\end{array}$ & 12.5 .5 & LOW & 1,2 \\
\hline $\begin{array}{l}\text { Phase separate the } \\
\text { raffinate from T- } 411\end{array}$ & CUB 4 & $\begin{array}{l}\text { The aqueous solution is transferred out ofT- } 411 \text { into a polyethylene bottle per the route in } \\
\text { Reference 12.3.2 above. }\end{array}$ & 12.5 .6 & \begin{tabular}{|l|} 
See Ref. \\
12.3 .2 \\
\end{tabular} & See Ref. 12.3 .2 \\
\hline $\begin{array}{l}2.0 \mathrm{M} \mathrm{NaBrO}_{3} \text { transfer } \\
\text { into T-411 }\end{array}$ & CUB 4 & Transferred into T-411 per the route in Reference 12.3.2 above (1st Scrub). & 12.6.1 & \begin{tabular}{|l|} 
See Ref. \\
12.3 .2 \\
\end{tabular} & See Ref. 12.3 .2 \\
\hline $\begin{array}{l}8 \mathrm{M} \mathrm{HNO}_{3} \text { transfer into } \\
\mathrm{T}-411\end{array}$ & CUB 4 & Transferred into T-411 per the route in Reference 12.3.2 above (1st Scrub). & 12.6 .2 & \begin{tabular}{|l|} 
See Ref. \\
12.3 .2
\end{tabular} & See Ref. 12.3.2 \\
\hline Mix in T-411 & CUB 4 & Previously discussed in Reference 12.5.4 above (1st Scrub). & 12.6 .3 & \begin{tabular}{|l|} 
See Ref. \\
12.5 .4 \\
\end{tabular} & See Ref. 12.5 .4 \\
\hline Settle in T-411 & CUB 4 & Previously discussed in Reference 12.5.5 above (1st Scrub). & 12.6 .4 & \begin{tabular}{|l|} 
See Ref. \\
12.5 .5 \\
\end{tabular} & See Ref. 12.5 .5 \\
\hline $\begin{array}{l}\text { Phase separate the } \\
\text { scrub from T-411 }\end{array}$ & CUB 4 & Previously discussed in Reference 12.3.2 above (1st Scrub). & 12.6 .5 & \begin{tabular}{|l} 
See Ref. \\
12.3.2
\end{tabular} & See Ref. 12.3 .2 \\
\hline $\begin{array}{l}2.0 \mathrm{M} \mathrm{NaBrO}_{3} \text { transfer } \\
\text { into T-411 }\end{array}$ & CUB 4 & Transferred into T-411 per the route in Reference 12.3.2 above (2nd Scrub). & 12.6.6 & \begin{tabular}{|l|} 
See Ref. \\
12.3 .2 \\
\end{tabular} & See Ref. 12.3 .2 \\
\hline $\begin{array}{l}8 \mathrm{M} \mathrm{HNO}_{3} \text { transfer into } \\
\mathrm{T}-411\end{array}$ & CUB 4 & Transferred into T-411 per the route in Reference 12.3.2 above (2nd Scrub). & 12.6 .6 & \begin{tabular}{|l|} 
See Ref. \\
12.3 .2 \\
\end{tabular} & See Ref. 12.3 .2 \\
\hline Mix in T-411 & CUB 4 & Previously discussed in Reference 12.5.4 above (2nd Scrub). & 12.6 .6 & \begin{tabular}{|l|} 
See Ref. \\
12.5 .4 \\
\end{tabular} & See Ref. 12.5.4 \\
\hline Settle in T-411 & CUB 4 & Previously discussed in Reference 12.5.5 above (2nd Scrub). & 12.6 .6 & \begin{tabular}{|l} 
See Ref. \\
12.5 .5 \\
\end{tabular} & See Ref. 12.5 .5 \\
\hline $\begin{array}{l}\text { Phase separate the } \\
\text { scrub from T-411 }\end{array}$ & CUB 4 & Previously discussed in Reference 12.3.2 above (2nd Scrub). & 12.6 .6 & \begin{tabular}{|l} 
See Ref. \\
12.3 .2 \\
\end{tabular} & See Ref. 12.3 .2 \\
\hline
\end{tabular}




\begin{tabular}{|c|c|c|c|c|c|}
\hline ITEM & LOCATION & DESCRIPTION & Reference & IMPACT & COMMENTS \\
\hline $\begin{array}{l}30 \% \mathrm{H}_{2} \mathrm{O}_{2} \text { transfer into } \\
\mathrm{T}-411\end{array}$ & CUB 4 & Transferred into T-411 per the route in Reference 12.3.2 above (1st Strip). & 12.7 .1 & $\begin{array}{l}\text { See Ref. } \\
12.3 .2\end{array}$ & See Ref. 12.3.2 \\
\hline $\begin{array}{l}8 \mathrm{M} \mathrm{HNO}_{3} \text { transfer into } \\
\mathrm{T}-411\end{array}$ & CUB 4 & Transferred into T-411 per the route in Reference 12.3.2 above (1st Strip). & 12.7 .2 & \begin{tabular}{|l|} 
See Ref. \\
12.3 .2 \\
\end{tabular} & See Ref. 12.3 .2 \\
\hline Mix in T-411 & CUB 4 & Previously discussed in Reference 12.5.4 above (1st Strip). & 12.7 .3 & $\begin{array}{l}\text { See Ref. } \\
12.5 .4 \\
\end{array}$ & See Ref. 12.5 .4 \\
\hline Settle in T-411 & CUB 4 & Previously discussed in Reference 12.5.5 above (1st Strip). & 12.7 .4 & $\begin{array}{l}\text { See Ref. } \\
12.5 .5 \\
\end{array}$ & See Ref. 12.5 .5 \\
\hline $\begin{array}{l}\text { Phase separate the } \\
\text { scrub from T-411 }\end{array}$ & CUB 4 & Previously discussed in Reference 12.3.2 above (1st Strip). & 12.7 .5 & \begin{tabular}{|l} 
See Ref. \\
12.3 .2 \\
\end{tabular} & See Ref. 12.3 .2 \\
\hline $\begin{array}{l}30 \% \mathrm{H}_{2} \mathrm{O}_{2} \text { transfer into } \\
\mathrm{T}-411\end{array}$ & CUB 4 & Transferred into T-411 per the route in Reference 12.3.2 above (2nd Strip). & 12.7 .6 & $\begin{array}{l}\text { See Ref. } \\
12.3 .2 \\
\end{array}$ & See Ref. 12.3 .2 \\
\hline $\begin{array}{l}8 \mathrm{M} \mathrm{HNO}_{3} \text { transfer into } \\
\mathrm{T}-411\end{array}$ & CUB 4 & Transferred into T-411 per the route in Reference 12.3.2 above (2nd t Strip). & 12.7 .6 & \begin{tabular}{l|} 
See Ref. \\
12.3 .2 \\
\end{tabular} & See Ref. 12.3 .2 \\
\hline Mix in T-411 & CUB 4 & Previously discussed in Reference 12.5.4 above (2nd t Strip). & 12.7 .6 & $\begin{array}{l}\text { See Ref. } \\
12.5 .4 \\
\end{array}$ & See Ref. 12.5.4 \\
\hline Settle in T-411 & CUB 4 & Previously discussed in Reference 12.5.5 above (2nd t Strip). & 12.7 .6 & \begin{tabular}{l|} 
See Ref. \\
12.5 .5 \\
\end{tabular} & See Ref. 12.5 .5 \\
\hline $\begin{array}{l}\text { Phase separate the } \\
\text { scrub from T-411 }\end{array}$ & CUB 4 & Previously discussed in Reference 12.3.2 above (2nd Strip). & 12.7 .6 & $\begin{array}{l}\text { See Ref. } \\
12.3 .2 \\
\end{array}$ & See Ref. 12.3.2 \\
\hline $\begin{array}{l}\text { Waste organic from T- } \\
411\end{array}$ & CUB 4 & $\begin{array}{l}\text { Waste organic is drained to a } 1 \mathrm{~L} \text { polyethylene bottle from Reference 12.1.1 above via the } \\
\text { route described in Reference 12.3.2 above. }\end{array}$ & 12.7 .7 & $\begin{array}{l}\text { See Ref. } \\
12.3 .2 \\
\end{array}$ & See Ref. 12.3 .2 \\
\hline Solution sampling & CUB 4 & $\begin{array}{l}\text { The raffinate solution, scrub solutions, waste organic solution, and product solution are } \\
\text { mixed and sampled using small volume pipettes. }\end{array}$ & 12.8 .1 & LOW & 1,2 \\
\hline $\begin{array}{l}\text { T-411 and T-409 flush } \\
\text { solution }\end{array}$ & CUB 4 & $\begin{array}{l}\text { Addition of prescribed concentrated } \mathrm{HNO}_{3} \text { acid and water to } \mathrm{T}-411 \text { to over flow into T-409 } \\
\text { per the route described in Reference 12.3.2 above. }\end{array}$ & 12.9 .1 & $\begin{array}{l}\text { See Ref. } \\
\text { 12.3.1.k } \\
\text { and 12.3.2 } \\
\end{array}$ & $\begin{array}{l}\text { See Ref. } \\
\text { 12.3.1.k and } \\
12.3 .2 \\
\end{array}$ \\
\hline Mix in T-411 & CUB 4 & Previously discussed in Reference 12.5.4 above. & 12.9 .2 & LOW & 1,2 \\
\hline $\begin{array}{l}\text { Drain flush solution } \\
\text { from T-411/T-409 to T- } \\
45\end{array}$ & $\begin{array}{l}\text { CUB 4, TK } \\
\text { PIT } 4\end{array}$ & Previously discussed in Reference 12.3.2 above. & 12.9 .3 & $\begin{array}{l}\text { See Ref. } \\
12.3 .2\end{array}$ & See Ref. 12.3 .2 \\
\hline Flush solution sample & CUB 4 & Grab sample pulled from end of T-411 drain line. & 12.9 .4 & LOW & 1,2 \\
\hline
\end{tabular}


13.0 Other Miscellaneous Equipment

\begin{tabular}{|c|c|c|c|c|c|}
\hline ITEM & LOCATION & DESCRIPTION & Reference & IMPACT & COMMENTS \\
\hline T-22 & $\begin{array}{l}\text { TK PIT 2, } \\
\text { MUA, CR }\end{array}$ & $\begin{array}{l}\text { Nominal } 68.7 \text { L tank, typically not frequently utilized for Cf Production Program } \\
\text { operations. }\end{array}$ & 13.1.1. & \begin{tabular}{|l|} 
See Ref. \\
13.1.2.a-e
\end{tabular} & $\begin{array}{l}\text { See Ref. } \\
\text { 13.1.2.a-e }\end{array}$ \\
\hline T-41 & $\begin{array}{l}\text { TK PIT 4, } \\
\text { MUA, CR }\end{array}$ & $\begin{array}{l}\text { Nominal 22.1 L tank, typically not frequently utilized for Cf Production Program } \\
\text { operations. }\end{array}$ & 13.1.1. & \begin{tabular}{|l|} 
See Ref. \\
13.1.2.a-e
\end{tabular} & \begin{tabular}{|l|} 
See Ref. \\
13.1.2.a-e
\end{tabular} \\
\hline T-42 & $\begin{array}{l}\text { TK PIT 4, } \\
\text { MUA, CR }\end{array}$ & $\begin{array}{l}\text { Nominal 22.1 L tank, typically not frequently utilized for Cf Production Program } \\
\text { operations. }\end{array}$ & 13.1.1. & \begin{tabular}{|l|} 
See Ref. \\
13.1.2.a-e
\end{tabular} & $\begin{array}{l}\text { See Ref. } \\
\text { 13.1.2.a-ee }\end{array}$ \\
\hline T-47 & $\begin{array}{l}\text { TK PIT 4, } \\
\text { MUA, CR } \\
\end{array}$ & $\begin{array}{l}\text { Nominal } 67.3 \text { L tank, typically not frequently utilized for Cf Production Program } \\
\text { operations. }\end{array}$ & 13.1.1. & \begin{tabular}{|l|} 
See Ref. \\
13.1.2.a-e \\
\end{tabular} & \begin{tabular}{|l|} 
See Ref. \\
13.1.2.a-e \\
\end{tabular} \\
\hline T-48 & $\begin{array}{l}\text { TK PIT 4, } \\
\text { MUA, CR }\end{array}$ & $\begin{array}{l}\text { Nominal 22.2 L tank, typically not frequently utilized for Cf Production Program } \\
\text { operations. }\end{array}$ & 13.1.1. & \begin{tabular}{|l|} 
See Ref. \\
13.1.2.a-e
\end{tabular} & \begin{tabular}{|l|} 
See Ref. \\
13.1.2.a-e
\end{tabular} \\
\hline T-67 & $\begin{array}{l}\text { TK PIT 6, } \\
\text { MUA, CR } \\
\end{array}$ & $\begin{array}{l}\text { Nominal 22.6 L tank, typically not frequently utilized for Cf Production Program } \\
\text { operations. }\end{array}$ & 13.1.1. & \begin{tabular}{|l|} 
See Ref. \\
13.1.2.a-e \\
\end{tabular} & \begin{tabular}{|l|} 
See Ref. \\
13.1.2.a-e \\
\end{tabular} \\
\hline T-71A & $\begin{array}{l}\text { TK PIT 7, } \\
\text { MUA, CR } \\
\end{array}$ & $\begin{array}{l}\text { Nominal } 64.3 \text { L tank, typically not frequently utilized for Cf Production Program } \\
\text { operations. }\end{array}$ & 13.1.1. & \begin{tabular}{|l|} 
See Ref. \\
13.1.2.a-e \\
\end{tabular} & \begin{tabular}{|l|} 
See Ref. \\
13.1.2.a-e \\
\end{tabular} \\
\hline T-73A & $\begin{array}{l}\text { TK PIT 7, } \\
\text { MUA, CR }\end{array}$ & $\begin{array}{l}\text { Nominal 22.1 L tank, typically not frequently utilized for Cf Production Program } \\
\text { operations. }\end{array}$ & 13.1.1. & \begin{tabular}{|l|} 
See Ref. \\
13.1.2.a-e
\end{tabular} & \begin{tabular}{|l|} 
See Ref. \\
13.1.2.a-e
\end{tabular} \\
\hline T-300 & $\begin{array}{l}\text { TK PIT 3, } \\
\text { MUA, CR }\end{array}$ & $\begin{array}{l}\text { Nominal } 22.0 \text { L tank, typically not frequently utilized for Cf Production Program } \\
\text { operations. }\end{array}$ & 13.1.1. & \begin{tabular}{|l|} 
See Ref. \\
13.1.2.a-e
\end{tabular} & \begin{tabular}{|l|} 
See Ref. \\
13.1.2.a-e
\end{tabular} \\
\hline T-301 & $\begin{array}{l}\text { TK PIT 3, } \\
\text { MUA, CR }\end{array}$ & $\begin{array}{l}\text { Nominal 22.4 L tank, typically not frequently utilized for Cf Production Program } \\
\text { operations. }\end{array}$ & 13.1.1. & \begin{tabular}{|l|} 
See Ref. \\
13.1.2.a-e
\end{tabular} & \begin{tabular}{|l|} 
See Ref. \\
13.1.2.a-e
\end{tabular} \\
\hline T-302 & $\begin{array}{l}\text { TK PIT 3, } \\
\text { MUA, CR } \\
\end{array}$ & $\begin{array}{l}\text { Nominal 22.1 L tank, typically not frequently utilized for Cf Production Program } \\
\text { operations. }\end{array}$ & 13.1.1. & \begin{tabular}{|l|} 
See Ref. \\
13.1.2.a-e \\
\end{tabular} & \begin{tabular}{|l|} 
See Ref. \\
13.1.2.a-e \\
\end{tabular} \\
\hline T-200 & $\begin{array}{l}\text { TK PIT 2, } \\
\text { MUA, CR } \\
\end{array}$ & Nominal 22.3 L tank, OOS due to leaking lines and or tank itself is leaking. & 13.1 .1 & N/A & OOS \\
\hline T-32 & $\begin{array}{l}\text { TK PIT 3, } \\
\text { MUA, CR }\end{array}$ & Nominal 68.3 L tank, OOS due to leaking lines and or tank itself is leaking. & 13.1 .1 & N/A & OOS \\
\hline
\end{tabular}




\begin{tabular}{|c|c|c|c|c|c|}
\hline ITEM & LOCATION & DESCRIPTION & Reference & IMPACT & COMMENTS \\
\hline Tank T-XX & $\begin{array}{l}\text { See Table in } \\
\text { Section 12.1 }\end{array}$ & $\begin{array}{l}\text { See Table in Section 13.1.1 for each tank volume, tank pit location, and material of } \\
\text { construction. }\end{array}$ & 13.1 .1 & HIGH & 11,12 \\
\hline $\begin{array}{l}\text { T-XX LL, DR, and PR } \\
\text { instr. }\end{array}$ & $\begin{array}{l}\text { See Table in } \\
\text { Section } 12.1\end{array}$ & $\begin{array}{l}\text { Foxboro pneumatic instrument is located in the CR and the DP cell and associated } \\
\text { polyethylene tubing is located in the MUA. The table in Section } \mathbf{1 2 . 1} \text { denotes the material } \\
\text { of construction of the LL, DR, and PR (if equipped with PR capability) lines and the tank } \\
\text { pits they originate. The instrument lines travel through the LAA, and are tied to the } \\
\text { polyethylene tubing that goes to the DP transmitters in the MUA. }\end{array}$ & 13.1.2.a & HIGH & $9,10,11,12$ \\
\hline T-XX sparger & CR, MUA & $\begin{array}{l}\text { There's a push button switch in CR to activate a solenoid in the MUA. An in-line orifice } \\
\text { controls air flow into the tank. }\end{array}$ & 13.1.2.b & LOW & $1,2,4$ \\
\hline $\begin{array}{l}\text { T-XX process and } \\
\text { instrument lines }\end{array}$ & $\begin{array}{l}\text { See Table in } \\
\text { Section 12.1 } \\
\end{array}$ & See Table in Section 12.1 for each tank and material of construction. & 13.1.2.c & HIGH & $9,10,11,12$ \\
\hline $\begin{array}{l}\text { T-XX vacuum } \\
\text { accumulator }\end{array}$ & $\begin{array}{l}\text { See Table in } \\
\text { Section } 12.1\end{array}$ & $\begin{array}{l}3 \text { in. diameter } \times 6 \text { in. long flanged glass pipe ( } \sim 1 \mathrm{~L} \text { volume) with specially fabricated } \mathrm{Zr} \text { top } \\
\text { and bottom plates. }\end{array}$ & 13.1.2.d & $\begin{array}{l}\text { NCBU or } \\
\text { OSS as } \\
\text { applicable } \\
\end{array}$ & $\begin{array}{l}\text { See Table in } \\
\text { Section } 12.1\end{array}$ \\
\hline SA-TXX needle block & $\begin{array}{l}\text { See Table in } \\
\text { Section } 12.1\end{array}$ & Specially fabricated Zr block with two needles for pulling samples. & 13.1.2.d & $\begin{array}{l}\text { NCBU or } \\
\text { OSS as } \\
\text { applicable } \\
\text { OSS }\end{array}$ & $\begin{array}{l}\text { See Table in } \\
\text { Section } 12.1\end{array}$ \\
\hline $\begin{array}{l}\text { Auxiliary glass sample } \\
\text { flask }\end{array}$ & $\begin{array}{l}\text { See Table in } \\
\text { Section 12.1 }\end{array}$ & $\begin{array}{l}1 \text { L glass bottle with 2-line stopper outfitted with polyethylene tubing connected to SA-T72 } \\
\text { position on CUB } 7 \text { sample rack for sampling T-72 and for connection to vacuum source. }\end{array}$ & 13.1.2.d & LOW & 1,2 \\
\hline $\begin{array}{l}\text { SA-TXX special 1-line } \\
\text { block }\end{array}$ & $\begin{array}{l}\text { See Table in } \\
\text { Section } 12.1 \\
\end{array}$ & $\begin{array}{l}\text { Special 1-line polyethylene block with 3/8 in. metal tubing on one side installed at SA-T72 } \\
\text { position on CUB } 7 \text { sample rack for sampling T-72 using auxiliary glass sample flask. }\end{array}$ & 13.1.2.d & LOW & 1,2 \\
\hline Tank T-10 & $\begin{array}{l}\text { TK PIT } 1, \\
\text { MUA, CR }\end{array}$ & $\begin{array}{l}\text { This tank is typically never used for process operations and is only used under special } \\
\text { approval. Originally used for storage of material for }{ }^{131} \text { I decay. }\end{array}$ & 13.2 .1 & N/A & N/A \\
\hline Tank Pit 5 tanks & $\begin{array}{l}\text { TK PIT 5, } \\
\text { MUA, CR }\end{array}$ & $\begin{array}{l}\text { With the exception of T-54, these tanks have never been used in support of the }{ }^{252} \mathrm{Cf} \\
\text { Program Operations. }\end{array}$ & 13.2 .2 & N/A & N/A \\
\hline $\begin{array}{l}\text { T-740, T-742, C-743 } \\
\text { and other LR } 7 \\
\text { equipment }\end{array}$ & $\begin{array}{l}\text { CUB 7, MUA, } \\
\text { CR }\end{array}$ & Originally used for the removal and retention of ${ }^{131} \mathrm{I}$, but no longer required to operate. & 13.2 .3 & N/A & N/A \\
\hline
\end{tabular}




\section{HOW TO INTERPRET THE IMPACT AND COMMENTS COLUMNS ON THE TABLE:}

\section{IMPACT}

OOS-Out of service

NCBU_-Not currently being used

HIGH-Significant impact to operational outcome and schedule

MED-Moderate impact to operational outcome and schedule

LOW-Little or insignificant impact to operational outcome and schedule

\section{COMMENTS}

1 Very little down time (typically $<1$ week) due to adequate spare parts inventory for this equipment and short lead time for procurement of approved replacement or repair parts.

2 Very little down time (typically $<1$ week) due to low number of man-hours required to repair/replace failed equipment.

3 Probability of failure is statistically low.

$4 \quad$ Alternative equipment maybe available to use as a substitute.

5 Moderate down time (typically $>1-4$ weeks) due to no spare parts inventory for this equipment and moderate lead time for procurement of approved replacement or repair parts. Moderate down time (typically >1-4 weeks) due to number of man-hours required to repair/replace failed equipment. Probability of failure is statistically moderate.

8 Alternative equipment maybe available to use as a substitute but will require moderate time and effort to put into service

9 Extensive down time (typically $>4$ weeks) due to no spare parts inventory for this equipment and long lead time for procurement of approved replacement or repair parts.

10 Extensive down time (typically $>4$ weeks) due to number of man-hours required to repair/replace failed equipment.

11 Probability of failure is uncertain or statistically high due to length of time in service.

12 Equipment is in a remote location or in an area with high radiological conditions, making it very difficult to repair.

13 Original equipment not in use. Alternative equipment being utilized.

14 Very low inventory of spares or no spares readily available. 
